This article was downloaded by: [California Institute of Technology]

On: 13 March 2009

Access details: Access Details: [subscription number 906391801]

Publisher Informa Healthcare

Informa Ltd Registered in England and Wales Registered Number: 1072954 Registered office: Mortimer House, 37-41 Mortimer Street, London W1T 3JH, UK

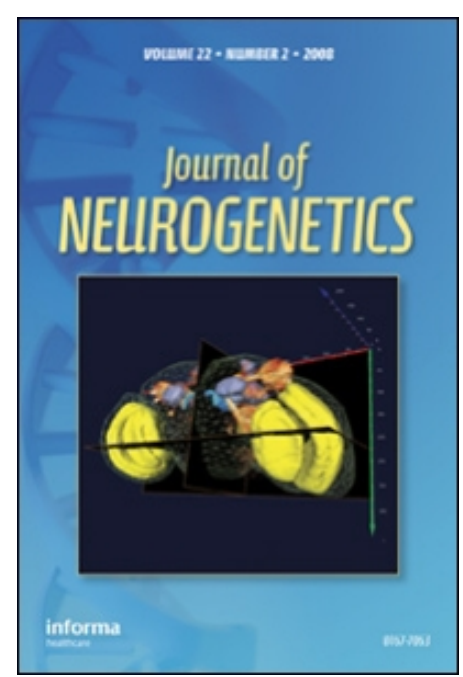

\title{
Journal of Neurogenetics
}

Publication details, including instructions for authors and subscription information:

http://www.informaworld.com/smpp/title content=t713644816

The 12th European Drosophila Neurobiology Conference 6-10 September 2008 Wuerzburg, Germany

Online Publication Date: 01 February 2009

To cite this Article (2009)'The 12th European Drosophila Neurobiology Conference 6-10 September 2008 Wuerzburg,

Germany',Journal of Neurogenetics,23:1,S1 - S102

To link to this Article: DOI: 10.1080/01677060902742364

URL: http://dx.doi.org/10.1080/01677060902742364

\section{PLEASE SCROLL DOWN FOR ARTICLE}

Full terms and conditions of use: http://www.informaworld.com/terms-and-conditions-of-access.pdf

This article may be used for research, teaching and private study purposes. Any substantial or systematic reproduction, re-distribution, re-selling, loan or sub-licensing, systematic supply or distribution in any form to anyone is expressly forbidden.

The publisher does not give any warranty express or implied or make any representation that the contents will be complete or accurate or up to date. The accuracy of any instructions, formulae and drug doses should be independently verified with primary sources. The publisher shall not be liable for any loss, actions, claims, proceedings, demand or costs or damages whatsoever or howsoever caused arising directly or indirectly in connection with or arising out of the use of this material. 


\section{Neurofly 2008 Abstracts}

\section{The 12th European Drosophila Neurobiology Conference 6-10 September 2008 Wuerzburg, Germany}

Conference Organizers: Martin Heisenberg and Erich Buchner, Department of Neurobiology and Genetics

University of Wuerzburg 


\section{LIST OF SESSIONS}

I Diseases and Aging

II Patterning and cell fate determination

III Differentiation of neurons and glia

IV Channels

V Synapse

VI Circuits

VII Behaviour

VIII Last Minute Communications

I.P

Ageing flies

Linda Partridge ${ }^{l}$

${ }^{1}$ Institute of Healthy Ageing, Dept of Genetics, Evolution and Environment, University College London

Recent years have seen a revolution in research into ageing, with the discovery of mutations in single genes that extend healthy lifespan. Drosophila has played and continues to play a key role in these discoveries. Evidence is accumulating that extension of lifespan is accompanied by a broad-spectrum improvement in health at later ages, and amelioration of specific ageing-related pathologies, including neurodegeneration.

I.1

The neurodegenerative mutant blue cheese links lipid metabolism with lysosomal function

Rachel Kraut ${ }^{1}$, Angeline Lim $^{2}$, Sarita Hebbar ${ }^{2}$

${ }^{1}$ IBN/A-STAR

${ }^{2}$ UC Santa Cruz

Many studies suggest a link between sphingolipid metabolism and neurodegeneration. The objective of our work is to understand the relationship between sphingolipid activity and neurodegeneration, using Drosophila genetics. We have evidence suggesting that the regulation of sphingolipid metabolism and trafficking in cells may be similar in Drosophila and mammals. We report on the characterization of blue cheese (bchs), a lysosomal protein with a highly conserved BEACH domain. This motif is required for sphingomyelinase (SMase) activation by the protein FAN in response to stress signals. Overexpression or loss of function of Bchs results in neurodegeneration accompanied by axonal transport defects and alterations in brain sterol levels and storage. Bchs' possible role in sphingomyelin cleavage raises the possibility that cholesterol storage is regulated by modulations in the levels of a SM-like lipid in the fly. Consistent with the biochemical data, genetic evidence links bchs-induced neurodegeneration to altered sphingolipid and cholesterol metabolism, with ceramide levels being the critical factor. Overexpression or loss of function in ceramidase or SMase modulate the degenerative phenotype. As a complement to the genetic and biochemical data, we are carrying out mass spectrometric analyses of sphingolipid alterations in bchs. In summary, the degenerative phenotype in bchs reveals mechanisms that interconnect sphingolipid and cholesterol regulation with neurodegeneration.

I. 2

Swiss Cheese, A Protein Involved In Neural Integrity, Neurite Outgrowth and Organophosphate Toxicity acts as a non-canonical regulatory subunit for PKA-C3.

Jill Wentzell ${ }^{1}$, Alexandre Bettencourt da Cruz ${ }^{1}$, Doris Kretzschmar $^{1}$

${ }^{1}$ Oregon Health \& Science University

The Drosophila swiss cheese (sws) mutant is characterized by progressive degeneration of the nervous system. Cell-specific expression reveals that neurons and glia depend autonomously on SWS expression. The human ortholog of SWS is Neuropathy Target Esterase (NTE), the molecular target in organophosphate-induced delayed neuropathy that is caused by many pesticides and chemical warfare agents. Recently mutations in NTE have been shown to cause a Hereditary Spastic Paraplegia called NTE-related Motor-Neuron Disorder. SWS and NTE are concentrated in the endoplasmic reticulum and both have been shown to have an esterase function against an artificial substrate. However, the functional mechanisms and the pathways in which SWS/NTE are involved in are still widely unknown. Yeast-two-hybrid experiments indicated that SWS interacts specifically with the C3 catalytic subunit of Protein Kinase A (PKAC3). Genetic interactions with deficiencies and PKA-C3 over-expressing lines support this finding and suggest 
that SWS acts as a non-canonical inhibitor of PKA-C3. In addition, we show that complex formation with the membrane bound SWS tethers PKA-C3 to membranes. We propose that improper regulation of PKA-C3 leads to the degenerative phenotype seen in the sws mutant and to organophosphate induced toxicity.

I.3

Quinone-based small molecules inhibit aggregation of Alzheimer disease-associated $\beta$-amyloid peptide (Aß) and alleviate its effects in flies

Roni Scherzer ${ }^{1}$, Michal Levy ${ }^{1}$, Anat Frydman-Marom ${ }^{1}$, Ehud Gazit $^{1}$, Daniel Segal ${ }^{1}$

${ }^{1}$ Biotechnology, Tel-Aviv University

Globular oligomers of the Alzheimer's disease-associated $\beta$-amyloid peptide $(A \beta)$ appear to be the major toxic species. Thus, an attractive therapeutic strategy for $\mathrm{AD}$ will be to block the early stage of misfolding and aggregation of soluble $\mathrm{A} \beta$ peptide. Recent work indicates that interactions between aromatic residues in the A $\beta$ peptide and other amyloidogenic proteins facilitate their aggregation. Small molecules harboring aromatic moieties may interfere with the protein-protein interaction and save as potent inhibitors of amyloids for therapy. A variety of quinones, natural or synthetic, are known to act as inhibitors of various metabolic paths in the cell and serve as antibacterial, anti-viral, and anticancer agents. Furthermore, danthron (1,8-dihydroxyanthraquinone) reduces neurotoxicity related to $\beta$-amyloid proteins. We have developed quinone-based molecules as potential amyloid inhibitors. Two such molecules, SY-81 and SY-83 were shown in vitro to prevent formation of toxic aggregates of the $A \beta_{1-42}$ and $A \beta_{1-40}$ peptides, in a dose dependent fashion, and prevent formation of $A \beta$ fibrils. They are non-toxic to cultured rat $\mathrm{PC} 12$ cells, and protect them from the deleterious effect of $A \beta_{1-42}$ and $A \beta_{1-40}$ at very low concentrations. When fed to transgenic Drosophila expressing the $A \beta_{1-}$ 42 fragment in their nervous system under Gal4 ${ }^{\text {elav }}$, these compounds dramatically alleviated their Alzheimer'sassociated locomotion (climbing) defects and life shortening, while having no effect on control flies.

I. 4

Dopamine-dependent neurodegeneration in Drosophila models of Parkinson's disease

Jose A. Botella ${ }^{1}$, Florian Bayersdorfer ${ }^{1}$, Aaron Voigt $^{2}$, Stephan Schneuwly ${ }^{2}$

${ }^{1}$ Lehrstuhl für Entwicklungsbiologie, University Regensburg
2 DFG Forschungszentrum "Molekularphysiologie des Gehirns". Universität Göttingen

Parkinson's disease has been found to be caused by both, genetic and environmental factors. Despite the diversity of causes involved in the progression of the disease, a convergent pathogenic mechanism might underlie the special vulnerability of dopaminergic neurons in different forms of Parkinsonism. In recent years, a number of reports have proposed dopamine as a common player responsible in the loss of dopaminergic neurons independently of its etiology. Using RNAi lines we were able to generate flies with drastically reduced dopamine levels in the dopaminergic neurons. Combining these flies with a chemically induced Parkinson model (Rotenone) and a familial form of Parkinson (mutant $\alpha$-synuclein) we were able to show a strong reduction of neurotoxicity and a rescue of the dopaminergic neurons when cellular dopamine levels were reduced. These results show that dopamine homeostasis plays a central role for the susceptibility of dopaminergic neurons to environmental and genetic factors in in vivo models of Parkinson disease.

I.5

Pan-neuronal expression of mutant $\alpha$-synuclein primarily impairs dopaminergic neurons in Drosophila

Thomas Riemensperger ${ }^{1}$, Marlène Cassar ${ }^{1}$, Hélène Coulom ${ }^{1}$, Magali Iché ${ }^{1}$, Serge Birman ${ }^{1}$

${ }^{1}$ Genetics of Neurotransmission, IBDML-Developmental Biology Institute, CNRS-Université de la Méditerranée, Campus de Luminy, Case 907, F-13288 Marseille Cedex 9, France

Parkinson's disease (PD), one of the most frequent neurodegenerative disorders, is related to progressive loss of dopaminergic neurons in subcortical nuclei. One of the genes implicated in an inherited form of PD is $\alpha$ synuclein ( $\alpha$-syn), a gene for which no homologue could be found in the Drosophila genome. Here, we studied phenotypic consequences of expression of human $\alpha$-syn and its mutant form $\alpha-\operatorname{syn}(\mathrm{A} 30 \mathrm{P})$ in Drosophila neurons. We found that pan-neural expression of $\alpha$-syn(A30P) with elav-GAL4 induced progressive locomotor impairments and a yet undescribed bang-sensitive paralysis phenotype. By targeting $\alpha$-syn(A30P) to dopaminergic or serotoninergic neuron subsets with cell-specific GAL4 drivers, we identified a single dopaminergic cluster that is likely responsible for these phenotypes. Furthermore, panneuronal expression of $\alpha$-syn(A30P) under paraquatinduced oxidative stress primarily triggered apoptosis in dopaminergic neurons, thus reducing fly life expectancy. In contrast, pan-neuronal expression of native $\alpha$-syn 
protected flies and dopaminergic neurons from the deleterious effects of paraquat. This suggests that $\alpha$ syn(A30P) specifically impedes dopaminergic neuron functionality and the resistance of these cells to oxidative stress in Drosophila.

I.6

\section{Pleiotropic roles of dopamine in ethanol-induced behaviors}

Kyung-An Han ${ }^{l}$, Hyun-Gwan Lee ${ }^{1}$, Young-Cho Kim ${ }^{1}$, David Moore ${ }^{1}$, Maryam Kherad Pezhouh ${ }^{1}$

${ }^{1}$ Biology, Pennsylvania State University

Ethanol affects various brain functions and its chronic use induces neural adaptation underlying alcoholism. To elucidate the cellular basis of ethanol's effects, we developed FlyTracker and Flypub. FlyTracker is a Kalman filter-based tracking system for monitoring the locomotor activating and depressing effects of ethanol while Flypub allows flies to get exposed to ethanol vapor in a mild manner. Upon daily ethanol treatment in Flypub, male flies display active intermale courtship, which represents a novel type of behavioral disinhibition. Moreover, ethanolexposed males develop behavioral sensitization, plasticity associated with drug addiction, since their intermale courtship activity is progressively augmented with additional ethanol experience. To investigate dopamine's roles in ethanol-associated behavior, we first tested the flies with temporally or chronically blocked dopamine neuronal activities. The flies with abnormal dopamine exhibit altered responses to ethanol in both FlyTracker and Flypub, suggesting that dopamine mediates multiple effects of ethanol. We explored the underlying mechanisms by testing mutants defective in dopamine receptors (dDA1, DAMB and dD2) and transporter. Notably, they show distinct phenotypes in the locomotor activating and depressing responses, behavioral disinhibition or sensitization. Studies are in progress to identify the neural sites that individual dopamine receptors and transporter mediate particular ethanol effects.

I.7

The dunce gene is required for ethanol tolerance in Drosophila melanogaster

Mirjam Franz ${ }^{1}$, Ansgar Klebes ${ }^{2}$, Anastasios Saratsis ${ }^{2}$, Henrike Scholz ${ }^{2}$

${ }^{1}$ University of Wuerzburg, Department of Genetics and Neurobiology, Am Hubland, 97074 Wuerzburg, Germany
2 FU-Berlin, Department of Genetics, Arnimallee 7, 14195 Berlin, Germany

To identify genes and cellular processes underlying ethanol tolerance we have previously established Drosophila melanogaster as a genetic model and uncovered that at least two different mechanisms contribute to tolerance. The phenotypic characterization of the hangover mutant shows that one mechanism bases on a cellular stress response. For a better understanding how cellular stress might change the function of neurons, we identified putative candidate genes of Hangover with a cDNA array screen. One putative target of Hangover is dunce. The dunce gene encodes a phosphodiesterase and regulates cAMP levels. We show that Hangover can bind dunce RNA in vitro. These observations are consitstent with the nuclear expression domains of Hangover. The phenotypic analysis of different dunce alleles indicate that a specific group of dunce transcripts is required for ethanol tolerance. Since the precise molecular nature of existing dunce mutants is not known, we generated a transcript specific dunce delta RMRA mutant. The dunce delta RMRA mutant develops reduced ethanol tolerance, is sensitive to cellular stress and has learning and memory defects. The dun$c e^{R M R A}$ specific promoter GAL4 line rescues the ethanol tolerance phenotype of dunce $e^{\text {deltal43 }}$ and hangover mutants. These results suggest that the RNA modifying protein Hangover might act through dunce modification inducing the development of tolerance in the adult brain.

The Fat tumor suppressor mediates neurodegeneration induced by the polyglutamine Atrophin via deregulation of autophagy.

Simona Occhi ${ }^{l}$, Bernard Charroux ${ }^{2}$, Piera Calamita ${ }^{1}$, Francesco Napoletano ${ }^{1}$, Ilaria Nisoli ${ }^{1}$, Vera Giulia Volpi ${ }^{1}$, Manolis Fanto ${ }^{1}$

1 Dulbecco Telethon Institute, DIBIT-San Raffaele Scientific Institute

${ }^{2}$ IBDML, Campus de Luminy, Marseille

It is becoming increasingly evident that two apparently unrelated processes like cancer and neurodegeneration disorders share an unexpected wealth of common biological mechanisms, the regulation of autophagy being an area of emerging overlap between the two phenomena. Among neurodegenerative diseases, polyglutamine pathologies combine general polyglutamine toxicity to disease-specific effects due to the proteins affected. Here we show that the fat tumour suppressor gene mediates neurodegeneration induced by the polyglutamine protein Atrophin via deregulation of autophagy. We demonstrate that in a Drosophila 
model of Dentatorubral-pallidoluysian Atrophy, human and Drosophila Atrophin bearing polyglutamine expansions induce autophagic neuronal degeneration. Through an unbiased approach we establish that Atrophin represses fat transcription and that this effect is enhanced by polyglutamine expansion. Modulation of fat levels modifies degeneration by Atrophin and homozygous fat clones promote progressive autophagic cellular degeneration. We finally report that this degeneration is partially mediated by the Hippo tumour suppressor pathway. Our data thus provide a crucial insight into the mechanism of a polyglutamine disease and uncover an unexpected neuroprotective role of Fat and the Hippo pathway in the regulation of autophagy and cellular homeostasis which links tumour suppression and neurodegeneration.

\section{11}

Transcriptional regulation of muscleblind, a gene involved in Myotonic Dystrophy

\author{
Llamusi Beatriz ${ }^{1}$, Garcia-Casado Zaida ${ }^{1}$, Artero Ruben ${ }^{1}$ \\ ${ }^{1}$ Department of Genetics, University of Valencia
}

Myotonic Dystrophy (DM) is an inherited multisystemic disease characterized by myotonia, muscular weakness, cardiac conduction defects, peripheral neuropathy and central nervous system (CNS) dysfunction. The genetic basis of the disease is the expansion of a CTG trinucleotide repeat in the 3'untranslated region of the $D M P K$ gene. Several lines of evidence support that mutant $D M P K$ transcripts are toxic to cells. Long CUG expansions fold into dsRNA hairpins that sequester nuclear proteins. Human alternative splicing factor MBNL1 aberrantly binds to long CUG expansions. Nuclear depletion of MBNL1 contributes to defects in a developmentally regulated switch from a fetal to adult alternative splicing pattern in selected muscle transcripts. The Drosophila homolog of MBNL1, Muscleblind, is expressed in tissues affected in DM such as the somatic and visceral musculature, eye retina, peripheral nervous system and embryonic and larval $\mathrm{CNS}$. We are interested in defining cis-regulatory sequences that control muscleblind expression in these tissues. Through bioinformatics and literature searches we defined five putative cis-regulatory sequences. The potential regulatory role of these regions is currently been assessed with the use of the GFP reporter in transgenic flies. Molecular epistasis experiments and biochemical assays to confirm DNA-protein interactions will be subsecuently performed.
I.12

Modelling Mental Retardation in Drosophila-A systematic approach to dissect molecular networks and mechanisms underlying human brain function and disease

Eiko K. de Jong ${ }^{1}$, Merel A.W. Oortveld ${ }^{1}$, Korinna Kochinke $^{1}$, Jamie M. Kramer ${ }^{1}$, Hans van Bokhoven ${ }^{1}$, Annette Schenck ${ }^{l}$

${ }^{1}$ Department of Human Genetics, Nijmegen Centre for Molecular Life Sciences, Donders Centre for Neuroscience, Radboud University Nijmegen Medical Center

We are interested in molecular networks and mechanisms underlying human brain function and disease. Mutations in more than 400 genes are known to give rise to Mental Retardation, providing an exciting starting point to study this problem. Some of these genes have been already shown to operate together to control specific aspects of nervous system development and function. We aim at systematically identifying such functional connections. In order to be able to investigate the large number of genes, we use the fruitfly Drosophila melanogaster as a model organism. In the fly, genes can be manipulated specifically in the nervous system and consequences for neuronal architecture, function and cognitive behaviour of the fly, such as learning and memory, can be studied and compared. Our long-term goal is to use the gained knowledge on mental retardation gene function and the fruitfly as a model to search for genetic and chemical modifiers of fly "mental retardation" phenotypes. We expect this research to provide conceptual advances in our understanding of molecular networks that wire the brain in health and disease and to significantly contribute to the identification of novel diagnostic and therapeutic strategies to MR disorders.

ER Stress and the Unfolded Protein Response in Photoreceptor Degeneration

Pedro Domingos ${ }^{1}$, Gen Joseph ${ }^{2}$, Hermann Steller ${ }^{2}$

${ }^{1}$ ITQB, Portugal/The Rockefeller University, USA

2 The Rockefeller University, USA

The endoplasmic reticulum (ER) is the cellular organelle where secretory and membrane proteins are synthesized and folded. The presence of unfolded/misfolded proteins in the ER causes stress to the cell ("ER stress") and activates the Unfolded Protein Response (UPR), a cellular 
response to restore homeostasis in the ER. The presence of misfolded proteins and activation of the UPR have been associated with many diseases and pathological conditions. We have previously established that the IRE1/ Xbp1 signaling pathway, an important component of the UPR, acts as a protector signal against retinal degeneration caused by the presence of misfolded mutant Rhodopsin 1 in the ER of photoreceptors. Here, we will present our current efforts to identify additional genes acting downstream to the IRE1/Xbp1 signaling pathway, by using conventional screening strategies in the Drosophila eye.

\section{I.14}

\section{Chemical modifiers of a CUG-induced neuronal phenotype in a Drosophila model of Myotonic Dystro- phy Type 1}

Amparo Garcia-Lopez ${ }^{1}$, Irma Garcia-Alcover ${ }^{2}$, M.Carmen Alvarez-Abril ${ }^{2}$, Ruben Artero ${ }^{1}$

${ }^{1}$ Department of Genetics, University of Valencia

2 Valentia biopharma

Myotonic dystrophy 1 (DM1) is an autosomal dominant neuromuscular disease involving the expansion of unstable CTG repeats in the 3' untranslated region of the DM protein kinase (DMPK) gene. Expression of mutant DMPK RNA is toxic to the cells and associates with formation of ribonuclear inclusions and late-onset degenerative changes in brain, heart or skeletal muscle, leading to effects on cognition, cardiac arrhythmias or myotonia, among others. A biochemical hallmark of DM1 is misregulated alternative splicing of specific pre-mRNAs of which brain-specific transcripts TAU, APP and NMDAR1 have been shown to be affected. To better understand the pathogenic effect of the toxic RNA we developed a Drosophila model expressing 480 interrupted CUG repeats in the context of a non-translatable RNA. These flies reproduced aspects of the DM1 pathology. Here we show that targeted expression of $480 \mathrm{CUG}$ repeats to the brain structure the mushroom bodies (MBs), but not $60 \mathrm{CUG}$ repeats, is toxic leading to semilethality in mature pupae. This phenotype was utilized to identify chemical modifiers of the CUGinduced toxicity. We found ten compounds that significantly improved viability of CUG expressing flies, which included non-steroidal anti-inflammatory agents; muscarinic, cholinergic and histamine receptor inhibitors, and drugs that can affect sodium and calcium metabolism.
These findings provide new insights into the DM1 phenotype, and suggest novel candidates for DM1 treatments.

\section{I.15}

\section{Rapamycin reduces neurotoxicity of a mutant form of $\alpha$-synuclein in Drosophila}

Florian Gmeiner $^{1}$, José A. Botella ${ }^{1}$, Stephan Schneuwly ${ }^{1}$

1 Lehrstuhl für Entwicklungsbiologie, Universität Regensburg

Toxic gain of function mutations and triplications of the gene $\alpha$-synuclein are known to induce early onset of Parkinson's disease. Although the mechanism underlying neurotoxicity of $\alpha$-synuclein is an issue of intense debate, it is generally accepted that the accumulation of soluble $\alpha$-synuclein oligomers is a central event involved in the loss of dopaminergic neurons. We have investigated the turnover of $\alpha$-synuclein in Drosophila and found a decreased degradation rate of the mutant familial form A30P, one of the most toxic forms of $\alpha$ synuclein in Drosophila. In order to study the relation between neurotoxicity and A30P degradation, flies were fed with Rapamycin, a potent activator of autophagy, and monitored for $\alpha$-synuclein degradation and dopaminergic neuronal cell loss. Our results indicate that Rapamycin restores the normal degradation rate of A30P and partially rescues dopaminergic neuronal cell loss providing new evidence for a direct correlation between altered $\alpha$-synuclein degradation and neurotoxicity in A30P implicating autophagy as an important process involved in removing toxic $\alpha$-synuclein accumulations.

Modelling Saposin Deficiency: a neurodegenerative lysosomal storage disorder in Drosophila.

Samantha Hindle ${ }^{l}$, Sean Sweeney ${ }^{1}$

${ }^{1}$ Biology, University of York

Mutations causing lysosomal accumulation of undegraded material (lysosomal storage disorders; LSDs) affect 1 in 8,000 live births. The most common result is childhood death, typically due to neurodegeneration. Despite its devastating implications, little is known about the signalling linking lysosomal storage to neurodegeneration. We hope to dissect these pathways using the powerful genetics 
of Drosophila. Saposins are sphingolipid activator proteins that facilitate the activity of sphingolipid hydrolases in the lysosome. There are 4 human saposins produced from a proprotein precursor encoded by the prosaposin gene. Mutation of each of these saposins causes a distinct LSD. Therefore, modelling saposin-deficiency in Drosophila using the prosaposin orthologue sap-r provides great scope for understanding a number of childhood neurodegenerative disorders. We have initiated a study of Drosophila sap$r$ gene function. The sap-r gene consists of 7 exons that encode a 953 amino acid protein with 6 predicted saposinlike domains. We have identified a P-element insertion in the 5' end of the sap-r locus and a PBac insertion in the open reading frame. Both insertions result in reduced longevity. P-element mobilisation to create null mutants yielded potential sap-r-deficient stocks displaying reduced longevity. Further phenotypic analysis focusing on neuronal pathology identifies neuronal vacuolarisation, a characteristic of LSD. This bodes well for sap-r mutants as a model for childhood neurodegeneration.

\section{18}

Neuroprotective effects of creatine against rotenone induced neurotoxicity and mitochondrial dysfunctions in Drosophila melanogaster

Ravikumar Hosamani ${ }^{1}$, Chandrashekar $\mathrm{KN}^{1}$, Muralidhara $\mathrm{M}^{1}$

${ }^{1}$ Biochemistry and Nutrition, Central Food Technological Research Institute

Recent studies have shown that dietary creatine $(\mathrm{Cr})$ supplementation has beneficial effects in models of acute/ chronic neurodegeneration, which are ascribed due to the bioenergetic, antiapoptotic, antiexcitotoxic and antioxidant properties. In the present study, we have attempted to obtain insights into the neuroprotective effects of $\mathrm{Cr}$ in a Drosophila model. The primary objective was to understand if $\mathrm{Cr}$ offers protection against Rotenone induced neurotoxic effects and basis of its neuroprotection. $D$. melanogaster (Oregon $\mathrm{K}$, adults) exposed to $\mathrm{Cr}$ (2, 5, $10 \mathrm{mM}$, diet) for 7 days showed no mortality, while the levels of lipid peroxidation were diminished (10-40\%) in whole body homogenates. Further, we investigated if $\mathrm{Cr}$ can protect against Rotenone (ROT) induced oxidative stress, locomotor deficits and lethality. While ROT, $(500 \mu \mathrm{M})$ caused significant mortality $(95 \%)$, co-exposure with $\mathrm{Cr}$ offered robust protection against ROT-induced lethality and locomotor deficits (by negative geotaxis). While $\mathrm{Cr}$ alone had no effect on fly behavior, it markedly ( $>50 \%$ ) improved the performance of ROT flies. In a satellite study, adult flies exposed to paraquat per se (40 $\mathrm{mM})$ showed significant mortality $(95 \%, 72 \mathrm{~h})$, while
$\mathrm{Cr}$ treated flies $(10 \mathrm{mM})$ exhibited higher survival rate $(50 \%, 72 \mathrm{~h})$ suggesting significant development of resistance. Our results demonstrate the potential neuroprotective effects of $\mathrm{Cr}$ in Drosophila, which are consistent with the hypothesis that $\mathrm{Cr}$ possesses significant beneficial effects against neurodegeneration.

\section{I.19}

\section{DLis1 and neurodegeneration}

Els Janssens ${ }^{1}$, Peter Carmeliet ${ }^{2}$, Patrick Callaerts ${ }^{1}$

1 Laboratory of Developmental Genetics, VIB and K.U.Leuven

${ }^{2}$ Vesalius Research Center, VIB and K.U.Leuven

Defective axonal transport has been implicated in various neurodegenerative diseases such as distal hereditary motorneuropathy type VII, which is caused by mutations in the human dynactin gene (DCTN1). We are studying the Drosophila dynactin homolog Glued to characterize a possible role in neurodegeneration of dynactin-interacting genes. One known $G l$-interacting gene is DLisl, the Drosophila homolog of LIS1. Mutations in human LIS1 are associated with classical lissencephaly, but no association has yet been found between LIS1 and motorneurodegeneration. To study this, we have first shown that heterozygosity for the dominant mutation in Glued $\left(G l^{1}\right)$ results in a progressive loss of motor performance. We are currently analyzing how altered DLisl levels affect the cellular and behavioral consequences of the $G l^{l}$ mutation. In an alternate approach, we use the GAL4-UAS system to overexpress mutated forms of DLis1, harboring mutations previously identified in human patients. This has revealed that the missense mutation $\mathrm{H} 149 \mathrm{R}$ encodes a dominant-negative version of the protein. Interestingly, targeted overexpression of H149R leads to defects in axonal projections and eye development very similar to those previously shown for heterozygous $G l^{l}$ flies. Furthermore, we have observed a progressive worsening of motor performance similar to the $G l^{l}$ neuromotor phenotype we found previously. We are currently analyzing the cellular and behavioral defects in more detail.

\section{High-throughput in vivo screen in a glioma model in Drosophila melanogaster}

Astrid Jeibmann ${ }^{1}$, Hanna Witte ${ }^{2}$, Christian Klämbt ${ }^{2}$, Werner Paulus ${ }^{1}$

\footnotetext{
${ }^{1}$ Institute of Neuropathology, University hospital Münster

${ }^{2}$ Institute of Neurobiology, University of Münster
} 
Many molecular pathways are highly conserved between invertebrates and humans. While fly models have been established for type 1 neurofibromatosis, tuberous sclerosis and neurodegenerative disorders, no fly model of glioma is available so far. Using the Gal4-UAS system we have recently established a Drosophila model overexpressing the constitutively activated $P v r(\lambda P v r)$ (homolog of the PDGF- and VEGF-receptor in Drosophila melanogaster) under control of the glia-specific promoter reversed polarity (repo) leading to markedly increased numbers of glial cells of larval eye imaginal disc, optic stalk glia and glial cells ectopically migrating along nerve tracts. Larval lines overexpressing activated $P v r$ showed fused brain lobes and thickened peripheral nerves. We take advantage of the fact that overexpression of $\lambda P v r$ leads to lethality at the late larval stages as this allows revealing interacting genes possibly involved in gliomagenesis by their potential of reverting the neuropathological phenotype and leading to survival of the flies. We are currently performing a high-throughput screen comprising $2000 \mathrm{RNAi}$ constructs and could already detect ten candidate genes, among these HistoneH3.3A (HisH3.3A), Toll-7 and lysyl oxidase like (lox).

\section{21}

\section{Serotonin modulates ethanol tolerance in Drosophila melanogaster}

Andrea Kaiser ${ }^{1}$, Yvonne Ritze, Maite Ogueta- Gutierrez, Jay Hirsh, Henrike Scholz

${ }^{1}$ Department of Genetics and Neurobiology, Biozentrum, University of Würzburg, Am Hubland, 97074 Würzburg

The disregulation of the neurotransmitter Serotonin (5HT) is associated with the development of high levels of tolerance and alcoholism in humans. However, the precise molecular mechanism how serotonin contributes to tolerance and alcohol dependence is unknown.

We utilize the fruit fly Drosophila melanogaster to investigate the role of 5HT in ethanol induced behaviors. The serotonin transporter (dSERT) plays a key role in regulating serotonin signaling. First we analyzed the expression pattern of the dSERT in adult flies with a newly generated dSERT antibody serum. The dSERT is especially expressed in all serotonergic neurons. Furthermore it is more widely expressed than 5HT. For example dSERT is expressed along axons whereas 5HT is mostly found in bouton- like structures. Additional, reduction of the dSERT function by pharmacological and genetic tools result in reduced ethanol tolerance. These findings suggest that local differences in 5HT concentration rather than absolute 5HT levels are important to mediate behavioral changes.
Our studies show that increased serotonin signaling influences the development of ethanol tolerance. Furthermore they suggest that proper regulation of serotonin signaling during the development of ethanol tolerance might be conserved between insects and higher vertebrates.

A genetic screen for axonal outgrowth and regeneration in Drosophila melanogaster

Marta Koch ${ }^{1}$, Matthew Morgan ${ }^{1}$, Marlen Schlieder ${ }^{1}$, Derya Ayaz ${ }^{1}$, Jiekun Yan ${ }^{1}$, Phillip Tate ${ }^{1}$, Bassem A. Hassan $^{1}$

${ }^{1}$ Laboratory of Neurogenetics, Department of Molecular and Developmental Genetics, VIB and Department of Human Genetics, KULeuven, Leuven, Belgium

Traumatic injury to the Central Nervous System (CNS) and neurodegenerative diseases are major medical concerns. Dysfunction and loss of neurons can result in devastating and irreversible sensory, cognitive, behavioural and motor deficits. Moreover the processes of neuronal circuit repair and axonal re-growth in the brain are severely limited. So far, genetic analysis in mice has identified a handful of genes that may aid regeneration of severed axons. However, there is yet to be a systematic search for genes capable of inducing axonal regeneration. Our laboratory has recently developed a physiologically robust approach for maintaining and performing precision injury in the Drosophila brain ex vivo. (Ayaz et al, 2008). By using the Lateral Neurons ventral (LNv) expressing GFP, we have performed a targeted overexpression screen to identify genes that affect neurite outgrowth in physiological conditions; reasoning that these genes would be the most likely to regulate regeneration. From this screen we have not only picked up genes already known to be involved in neurite outgrowth, such as members of the Rho family of GTPases, but also genes from pathways not yet associated with neurite outgrowth. The positive hits are currently being validated and being further analysed in the injury model. Our objective is to uncover novel genes and pathways that will be promising targets for therapeutic intervention after CNS injury.

Oxidative stress reveals anti-aging protective role of period in Drosophila melanogaster

Natraj Krishnan ${ }^{1}$, Jadwiga Giebultowicz ${ }^{1}$

${ }^{1}$ Zoology, Oregon State University 
Circadian clocks are key adaptations that allow animals to anticipate daily environmental changes and regulate developmental and physiological processes. It is unclear how perturbations of clock mechanism affect longevity. Aging is associated with increased levels of reactive oxidative species giving rise to the "free radical theory of aging". Though this theory of aging has received considerable support, yet the mechanisms of aging are not well understood. Here, we have explored the nexus between clock and longevity in response to oxidative stress in Drosophila melanogaster. We found that the lack of per gene function, a core component of the clock system, does not seem to have dramatic impact on longevity of Drosophila males under normal laboratory conditions. However, the longevity of male period mutants was significantly reduced compared to their wild-type counterparts when males were exposed to oxidative stress (100\% hyperoxia) in middle or advanced age. Our data suggest that the decreased longevity in per mutants is associated with increased accumulation of carbonylated proteins as well as decreased elimination of damaged proteins by proteasome in flies of advanced age. Taken together our data suggest that PER protein confers a protective role in maintaining protein homeostasis during aging and lack of it leads to impaired longevity under conditions of stress. We currently investigate whether increased susceptibility of aging flies to oxidative stress is due to disrupted clock or to pleiotropic effects of per gene.

\section{Rescue and gene silencing experiments to dissect the DmMANF mutant lethality}

Riitta Lindström ${ }^{1}$, Mari Palgi ${ }^{1}$, Mart Saarma ${ }^{1}$, Tapio I Heino $^{2}$

\footnotetext{
${ }^{1}$ Institute of Biotechnology, University of Helsinki

${ }^{2}$ Institute of Biotechnology and Department of Biological and Environmental Sciences, University of Helsinki
}

A novel mammalian neurotrophic factor, MANF (Mesencephalic Astrocyte-derived Neurotrophic Factor) (Petrova et al. 2003, J Mol Neurosci 20:173-188), selectively promotes the survival of dopaminergic neurons in vitro. CDNF (Conserved Dopaminergic Neurotrophic Factor), a paralogous protein for MANF, promotes and repairs nigrostriatal dopaminergic system in a rat model of Parkinson's disease (PD) (Lindholm et al. 2007, Nature 448:73-77) thus representing a potential drug for treatment of PD. These proteins form the first family of neurotrophic factors with well conserved protein sequences through evolution among the multicellular organisms from C. elegans to human.

We have identified the fly homologue to mammalian MANF and CDNF, DmMANF, and generated null mutants of DmMANF which die as early second instar larvae (Palgi et al., submitted). This lethality was rescued by ubiquitous expression of DmMANF transgenes, and our rescue experiments also showed that human MANF but not CDNF is able to substitute the lack of endogenous DmMANF protein confirming MANF as the functional human orthologue of the fly protein. We designed rescue and RNAi experiments based on the expression pattern of DmMANF to find out the critical tissues for survival. Here we report how different ectopic expression of DmMANF rescues the lack of endogenous DmMANF and how gene silencing using RNAi fly line in broad range of expression patterns alters the fly development.

\section{A virtual fly ear for analyzing the consequences of mutant defects in hearing}

Simon Q. Lu ${ }^{1}$, Björn Nadrowski ${ }^{1}$, Jörg T. Albert ${ }^{2}$, Martin C. Göpfert ${ }^{l}$

${ }^{1}$ Sensory Systems Lab, Institute of Zoology, University of Cologne

${ }^{2}$ UCL Ear Institute, London

The antennal hearing organ of Drosophila provides a valuable system to genetically dissect hearing, yet how auditory relevant genes functionally contribute to the process of hearing largely remains unclear. To facilitate routine characterizations of auditory defects in Drosophila mutants, we have developed a virtual fly ear that allows to relate alterations in the ear's macroscopic performance to alterations in molecular events. The core of this virtual ear is a physical model with two opposed populations of transduction modules that couple to a harmonic oscillator that represents the fly's antennal sound receiver. The model can be accessed via a user interface that includes fitting algorithms as well as stimulation, simulation, and analysis tools. The virtual ear can be used to analyze experimental data and extract molecular parameter values according to the core model. Alternatively, the virtual ear allows for the in silico exploration of possible phenotypes that can result if molecular parameters change. The virtual ear will be 
made freely accessible and can be used as a ready-to-use research and teaching tool to explore fundamental mechanisms in hearing.

\section{In vivo biotinylation of neuronal ubiquitin conjugates}

Ugo Mayor $^{1}$, Andrea Brand ${ }^{1}$

${ }^{1}$ WT CR UK Gurdon Institute, University of Cambridge

Ubiquitination has been implicated in regulating axonal growth and path finding, presynaptic growth, postsynaptic density composition, synaptic efficacy, synaptic plasticity, and axonal degeneration. Ubiquitination disorders have been linked to many neurodegenerative conditions, including Huntington and Alzheimer's diseases. With a few exceptions, the regulatory mechanisms by which neuronal proteins are targeted for ubiquitination are poorly understood. We aim to identify the neuronal substrates of the ubiquitin proteasome system, to further understand the molecular basis of neuronal plasticity. The isolation of ubiquitin conjugates at a proteomic scale has been successfully achieved in yeast and in tissue culture. We have developed a novel strategy for tissue-specific expression of in vivo biotinylated ubiquitin. Ubiquitin conjugates can then be purified under denaturing conditions in order to minimize the amount of copurifying proteins. Our ubiquitin construct is functional as it is incorporated into conjugates when expressed in Drosophila embryonic neurons. It is biotinylated in the living embryo with a very high efficiency, and the ubiquitin conjugates bind strongly to the streptavidin beads. We are planning to identify the ubiquitin conjugates isolated this way using Mass Spectrometry in collaboration with Junmin Peng' lab (Emory University, Atlanta), and also to determine which of them are no longer ubiquitinated in flies mutant to specific E3 ligases.

\section{28}

Fos activation and synaptic growth in a Drosophila model of Lyososomal Storage Disease

Valerie Milton $^{1}$, Kate Gowers ${ }^{1}$, Sean T. Sweeney ${ }^{1}$

${ }^{1}$ Department of Biology (Area 9), University of York

We have previously identified the mutation spinster as a Drosophila model of Lysosomal Storage Disease (LSD). LSD is the most common form of childhood neurodegeneration and occurs in 1 in 8000 livebirths. Hallmarks of the disease include swollen lysosomes, accumulation of lipofuscin, excessive synaptic/dendritic growth and short lifespan. These symptoms are elicited upon the loss of proteins involved in lysosomal function from 45 different loci. Spin encodes a late endosomal/lysosomal protein, loss of which results in lipofuscin accumulation, swollen lysosomes, short lifespan and excessive synaptic growth making it an excellent model for LSD. The molecular events generating the pathogenic aspects of LSD remain to be determined. Here, we identify activation of the transcription factor Fos as a major determinant driving the synaptic overgrowth observed in spin. A role for Fos in the presynaptic regulation of synapse growth has been well documented. In spin and in wild type larvae, we find that Fos is present in motorneurons and muscle. We completely rescue synaptic overgrowth by expression of a Fos dominant negative (DN) transgene pre- and post-synaptically similtaneously. A Jun DN transgene has no effect. Using muscle or neuronal drivers, we find that $50 \%$ of the overgrowth is contributed by each compartment. Driving a wildtype Fos transgene in muscle generates no additional synaptic growth suggesting the presence in spin muscles of an additional factor allowing synaptic growth.

\section{I.29}

The Drosophila $\beta$-Amiloid Precursor Protein (APPL) protects against vap-dependent neurodegeneration

Ignacio Monedero ${ }^{1}$, Jose A. Botella ${ }^{2}$, Begoña Lopez ${ }^{1}$, Laura Torroja ${ }^{1}$

${ }^{1}$ Biology, Universidad Autonoma de Madrid

${ }^{2}$ Lehrstuhl für Entwicklungsbiologie. Universität Regensburg

The Drosophila vap (vacuolar peduncle) mutant is defective in the RasGAP gene, which acts as modulator of the Ras signaling pathway. vap mutants are characterized by progressive neurodegeneration in the optical lobes and central complex of the adult nervous system. Gene expression profiling of vap mutants showed increase expression of the Appl gene, the Drosophila homolog of the human $\beta$-amyliod precursor protein (APP) involved in Alzheimer's disease. Previous studies related APPL with synaptic development (Torroja et al. 1999) and axonal outgrowth (Leyssen et al. 2005). Thus, we studied the possible connection between APPL and the neurodegenerative process of vap mutants. We compared neurodegeneration in vap mutant fly brains, vap mutants carrying a deletion of the Appl gene $\left(\mathrm{Appl}^{d}\right)$, and vap mutants overexpressing APPL in neurons. Our results show that neuronal overexpression of APPL strongly reduces vapdependent neurodegeneration. However, the vap-dependent neurodegenerative process is not modified in $A p p l^{d}$ vap double mutants. Because $\mathrm{Appl}^{d}$ flies die prematurely after traumatic brain injury (Leyssen et al. 2005), we measured life span in mutant combinations of vap and Appl. We detect a decrease of the maximum life span in 
vap mutants carrying a deletion in Appl gene compared to vap mutants. Our data suggest that APPL protects against vap-dependent neurodegeneration.

\section{Expression pattern of Drosophila Septin 4, a potential Parkin substrate}

Veronica Muñoz-Soriano $^{l}$, Josep Navarro-López ${ }^{1}$, Nuria Paricio $^{1}$

${ }^{1}$ Department of Genetics, University of Valencia

In humans, parkin loss-of-function mutations are linked to autosomal recessive juvenile parkinsonism (ARJP). parkin is an E3 ubiquitin ligase that promotes degradation of specific target proteins by the proteasome. It has been proposed that loss of parkin activity will result in accumulation of its substrates, thus leading to the characteristic dopaminergic (DA) neuron death observed in patients. In Drosophila, parkin mutations cause degeneration of a subset of DA neurons in the brain, thus recapitulating one of the Parkinson's disease related phenotypes. Septin 4 is the Drosophila ortholog of human septin 5, the first parkin substrate identified in humans. We recently showed that Septin 4 overexpression caused age-dependent DA neuron degeneration in a specific dopaminergic cluster of the adult brain, and that this neurotoxicity was modified by changes in parkin function. Taken together, these results suggested that Septin 4 could be a substrate of Drosophila Parkin in vivo, which is supported by the fact that both proteins interact physically in co-precipitation assays. To further characterize the Septin 4 protein and its relation to Parkin, we have developed an anti-Sep4 antibody. We will present the results of Septin 4 localization in different tissues as well as the analysis of Septin 4 levels in different genotypes, including parkin mutants.

\section{I.31}

Glial and Central Nervous System neurodegeneration in a Drosophila model of Friedreich's ataxia

Juan Antonio Navarro ${ }^{l}$, Jose Antonio Botella ${ }^{1}$, Jose Vicente Llorens ${ }^{2}$, Maria Dolores Moltó ${ }^{2}$, Stephan Schneuwly ${ }^{1}$

1 Lehrstuhl für Entwicklungsbiologie, University of Regensburg

${ }^{2}$ Departament de Genètica. Universitat de València

Friedreich ataxia (FA), the most common form of hereditary ataxia, is a recessive neurodegenerative disease affecting the peripheral and central nervous systems. The pathological hallmarks of the disease include on the one hand destruction of the ganglia of dorsal spinal roots and degeneration of the longest and largest myelinated fibres of the spinal cord and on the other hand atrophy of dentate nucleus of the cerebellum and mild neuronal loss in the cerebellar cortex and degeneration of the cortical-spinal motor tracts. Frataxin, the gene affected in FA patients, encodes a conserved protein found ubiquitously in prokaryotes and eukaryotes. In order to mimic in Drosophila the scenario in the nervous system of the patients, we have reduced the expression of the Drosophila frataxin homolog gene ( $f h$ ) using the UAS-GAL4 system and the RNAi flies developed by Anderson et al. in different nervous system tissues (panneural, glial cells, motorneurons). In all cases, reduction of frataxin expression led to increased sensitivity to oxidative stress, reduded lifespan, an impairment of the locomotor activity and an agedependent neurodegeneration phenotype. Using ElectronMicroscopy we could show the presence of putative lipid accumulation in the glial cells. These results clearly indicate a key role for frataxin in the maintenance of the drosophila nervous system and suggest a metabolic problem underlying frataxin deficiency.

Tau accumulation and its phenotypic consequences depend on the abundance of 14-3-3 isoforms

Aikaterini Papanikolopoulou ${ }^{l}$, Sofia Grammenoudi ${ }^{1}$, Efthimios Skoulakis ${ }^{1}$

\section{${ }^{1}$ BSRC Alexander Fleming}

Many neurological disorders, including $\mathrm{AD}$ and the frontotemporal dementias, are characterized by the presence of intraneuronal inclusions made of abnormally phosphorylated forms of the microtubule-binding protein, tau. Moreover, it has been reported that the abundance of 14-3-3 proteins in particular brain regions and the cerebrospinal fluid of patients with $\mathrm{AD}$ is significantly increased. 14-3-3 proteins are signalling molecules, abundant in the nervous system, crucial for various physiological cellular processes. In this study, we use the simplicity and genetic facility of the Drosophila system with only two 14-3-3 genes to investigate in vivo whether 14-3-3s are causal or synergistic with tau accumulation to precipitate pathology. We present data demonstrating that elevation of either of the two Drosophila 14-3-3s, zeta or epsilon, throughout the CNS significantly increases the levels of wild-type tau. In contrast, similar co-expression with FTDP-17 linked mutant taus yields differential effects depending on the mutation. That implies that wild-type and mutant tau isoforms interact with the same proteins in different ways, potentially with distinct consequences. Importantly, the nature of mutation plays 
a significant role in its interaction with the 14-3-3s. Our results indicate that the two members of the Drosophila 14-3-3 protein family, exhibit distinct types of interactions with particular wild-type and mutant tau proteins.

Cdk5, but not its standard activator p35, is needed for Learning and Memory in Drosophila

Nina Pettersson ${ }^{l}$, Beat Suter ${ }^{1}$

${ }^{1}$ Institute of Cellbiology', University of Bern

Cdk5 is a protein kinase with a broad range of substrates, consequently involved in many different cellular processes such as development of the central nervous system (CNS), regulation of the cytoskeleton, axon guidance, membrane transport, synaptic function and associative learning. Deregulation of Cdk5 has been implicated in neurodegenerative disorders such as Alzheimer's disease (AD), Amylotrophic Lateral Sclerosis (ALS) and Parkinson's disease.

In mice, Cdk5 is activated by p35 and p39, and their cleavage products $\mathrm{p} 25$ and p29. p25 increase Cdk5 activation and shifts Cdk5 localization from the plasma membrane to the cytosol. High levels of p25 are connected with neurodegenerative diseases. Interestingly, prolonged expression of p25 decrease learning and memory in mice, while transient expression has been shown to improve memory.

We have confirmed that Cdk5 is needed for learning and memory by using a larva learning assay. This study also revealed that the Cdk5 dependent learning and memory seems to be independent of its activator p35. We are further investigating which other proteins might be required for this process through interacting studies. Moreover, we are examining the role of increased levels of these proteins on learning and memory by studying transgenic flies overexpressing Cdk5 and/or its activators p35/25 in the nervous system.

I.34

\section{Expression profiling of Johnston's organ reveals Drosophila deafness genes}

Pingkalai Rajeswaran ${ }^{1}$, Guvanch-Murad Ovezmuradov ${ }^{1}$, Thomas Effertz ${ }^{1}$, Martin C. Göpfert ${ }^{l}$

${ }^{1}$ Sensory Systems Lab, Institute of Zoology, University of Cologne

Hearing in Drosophila relies on Johnston's organ in the $2^{\text {nd }}$ segment of the antenna. The mechanosensory neurons of this organ and vertebrate inner-ear hair cells are developmentally specified by homologous genes (fly atonal, mouse atonal homologue Mathl). To identify candidate genes for hearing, we compared gene expression in the $2^{\text {nd }}$ antennal segments of atonal mutants and controls. Ca. 275 genes are identified that are preferentially expressed in JO. These genes include 4 known JO genes, 8 known ciliary compartment genes, and genes implicated in intracellular signalling (19 genes), protein modification (25 genes), nucleic acid regulation (23), cell metabolism (9 genes), transport (12 genes), and sensory perception (18 genes). 13 genes encode cytoskeletal components, 17 encode ion channels, and 13 molecular motors. Every $7^{\text {th }}$ gene identified by our screen seems related to a candidate human deafness gene. Ongoing functional analyses show that disrupting genes identified by our screen affects fly hearing.

\section{I.35}

\section{Dissection of the role of Octopamine in ethanol} induced behavior

Andrea Schneider ${ }^{1}$, Osman Cibic ${ }^{1}$, Katrin Volkmann ${ }^{1}$, Uschi Werner $^{1}$, Stefanie Hampel ${ }^{1}$, Henrike Scholz ${ }^{1}$

${ }^{1}$ Genetics and Neurobiology, University of Wuerzburg, Am Hubland, 97074 Wuerzburg, Germany

In the CNS of invertebrates the biogenic amines Tyramine and Octopamine are important neurotransmitters. We have previously shown that mutants with altered Tyramine and Octopamine levels show alterations in their ethanol induced behaviors. The incactive mutants with the reduced Tyramine and Octopamine levels show increased ethanol sensitivity, an impaired startle response and defects in the regulation of alcohol induced hyperactivity. The detailed analysis of this mutant revealed a role for Octopamine in mediating the odor evoked initial startle response and for Tyramine in the regulation of ethanol induced hyperactivity. The Tyramine- $\beta$-hydroxylase mutants $(\mathrm{T} \beta \mathrm{H})$ fail to synthesize Octopamine and develop a defect in the initial startle response, the regulation of ethanol induced hyperactivity, sedation and ethanol tolerance. Furthermore Octopamine is also known to play an important role in learning and building the appetitive memory (Schwaerzel et al., 2003). Taken together, Octopamine is important for the fly to respond correctly to external stimuli.

For a better understanding of the function of Octopamine in ethanol induced behaviors we have developed immunohistochemical and molecular genetic tools that allow us to alter and monitor Octopamine expression in the fly brain. We are now able to manipulate different sets of octopaminergic neurons in the brain with the UAS/GAL4 system and address the consequences of these alterations for ethanol induced behvaviors. 
I.36

\section{Drosophila melanogaster - a model system for protein aggregation}

Sebastian Schultz ${ }^{1}$, Gunilla Westermark ${ }^{1}$

1 Department of Clinical and Experimental Medicine, Diabetes Research Centre, University of Linköping, 58185 Linköping, Sweden

Recent research supports that aggregation of islet amyloid polypeptide (IAPP) leads to cell death and makes it a plausible cause for the halving of beta cell mass that occurs in patients with type 2 diabetes. IAPP and insulin are both produced as prohormones and processed into biological active hormones in the secretory granules by the convertases $\mathrm{PC} 1 / 3$ and $\mathrm{PC} 2$, prior to their stimulated co-secretion. In vitro, IAPP is one of the most amyloidogenic peptides known, while the presence of insulin can inhibit IAPP-fibril propagation. It is unclear if the intragranular equilibrium of prohormones and their putative processing metabolites affect amyloid formation. At present, we are in progress to establish a Drosophila melanogaster model that will allow studies of this. Here we present the results of proIAPP, IAPP and proinsulin expression in 7 different Gal4 lines. The influence was assayed by longevity at $26^{\circ} \mathrm{C}$ for males and mated females separately. Neuronal peptide expression with Gal4elavC155 led to a reduction of median survival compared to controls (25-37\% in females; $15-27 \%$ in males). Interestingly, the use of strong Gal 4 expression (Gal4 GMR) was not suitable since the lethal effect of Gal 4 expression alone outnumbered the lethal effect of proIAPP or proinsulin expression. Conclusion: Hence the usability of Drosophila melanogaster for studies of IAPP aggregation is influenced by $\boldsymbol{i}$ the site of expression, $\boldsymbol{i} \boldsymbol{i}$ strength of Gal4 expression and iii the sex of the flies.

\section{I.37}

Functional analysis of neurodegenerative mutant 4.14.10 of Drosophila melanogaster

Oksana Shcherbakova ${ }^{1}$, Dariya Maksymiv ${ }^{1}$, Yaroslava Chernyk $^{1}$, Stephan Schneuwly ${ }^{2}$

1 Department of Genetics and Biotechnology, Lviv National University of Ivan Franko, Ukraine

${ }^{2}$ Institute of Zoology, University of Regensburg, Germany

The isolation and characterization of Drosophila neurodegenerative mutants provide valuable insights into genes, pathways, and mechanisms causing neurodegeneration. A saturation mutagenesis screen with chemical mutagens was performed and 15 mutant lines of D. melanogaster on the $3^{\text {rd }}$ chromosome were obtained. Flies show a reduced lifespan and age-related degeneration in brain. After hyperoxia treatment one line, 4.14.10, showed a new phenotype, not found in any other mutant. Without hyperoxia, pathology in brain tissue in this mutant appears at the central parts of the brain after 20 days of imago life, but after 5 days of hyperoxia mutant flies showed severe degeneration of lamina cortex, vacuolization of retina, the space between lamina and eye filled with pigment granules from retina. Flies 4.14.10 also characterized by partial degeneration of photoreceptor axons R7-R8, reduced levels of phosphatidyl inositol and ergosterol, and behavioral derangements.

According to the crosses with flies from the Bloomington Stock Center which had deletions in the regions of known neurodegenerative mutants, the mutation 4.14.10 could be mapped in the cytological region of the neurodegenerative mutant loechrig. Complementation test of mutant 4.14 .10 with loe flies showed neurodegeneration in progeny that indicate that mutation 4.14.10 is an allele of known gene loechrig.

The Drosophila dopaminergic system: characterisation and application as a model for Parkinson's disease

Katherine White ${ }^{1}$, Dickon Humphrey ${ }^{1}$, Frank Hirth ${ }^{1}$

1 MRC Centre for Neurodegeneration Research, King's College London

Parkinson's disease (PD) is the most common neurodegenerative movement disorder, characterised by the progressive loss of dopaminergic (DA) neurons. We have established baseline parameters relevant to the application of Drosophila as a model for PD, including DA neuron survival and locomotor behaviour throughout lifespan. We characterise in detail the projection pattern of DA neurons expressing GFP in the adult Drosophila brain, with special emphasis on projections to the central complex, which is described as a centre for the higher control of locomotion. Based on these parameters, we have tested the prevalent hypothesis that DA neurons may be vulnerable to degeneration in PD because dopamine itself can readily oxidise to generate damaging reactive oxygen species. Adult flies have been chronically exposed to reserpine, a drug which blocks the uptake of dopamine into synaptic vesicles by inhibiting the vesicular monoamine transporter (VMAT). Ageing flies show a dose-dependent decline in locomotion, but no reduction in lifespan or loss of DA neurons compared to controls. The reserpine-induced locomotor phenotype can be partially rescued by DA neuron-specific overexpression 
of DVMAT. Enhancing dopamine synthesis by overexpression of the rate-limiting enzyme tyrosine hydroxylase did not affect the survival of these cells, even in combination with reserpine treatment. We conclude that misregulation of cellular dopamine alone is insufficient to induce neurodegeneration in this model.

I.41

Social interaction-mediated lifespan extension and electrophysiological correlates in Drosophila $\mathrm{Cu} / \mathrm{Zn}$ superoxide dismutase mutants

Chun-Fang $W u^{l}$, Hongyu Ruan ${ }^{1}$

${ }^{1}$ Department of Biology, University of Iowa

Beneficial effects of social interaction on aging have been studied in humans and other species. We found that shortlived Sod mutants, defective in the anti-oxidant enzyme $\mathrm{Cu} / \mathrm{Zn}$ superoxide dismutase, displayed a robust lifespan extension, with improved stress resistance and motor ability, upon co-housing with active flies of longer lifespan or younger age. Genetic, surgical and environmental manipulations revealed motor and sensory components in behavioral interactions required for the lifespan extension by co-housing (PNAS 105: 7506, 2008). We examined the electrophysiological properties of the compound eyes, giant fiber (GF) circuit, and flight muscle DLMs in control and co-housed Sod flies. Compared to wild type (CS), Sod flies showed normal basal GF pathway properties, including long- and short-latency responses (LLR, SLR) but displayed striking alterations in activity-dependent plasticity, including, longer refractory and faster habituation of LLR and lowered following frequency of SLR. In parallel to diminished electroretinogram and weakened muscle physiology, Sod flies were less susceptible to electroconvulsion-induced seizure, indicating lowered excitability. Preliminary results indicate that co-housing improved DLM resting and action potentials (in $\mathrm{mV}, \mathrm{RP}:-78.1 \pm 2.7 \mathrm{vs}$. $-68.2 \pm 1.9$, and AP: $49 \pm 2.6$ vs. $38.2 \pm 3.7$, at $50 \%$ lifespan). Further studies will reveal the underlying molecular networks and physiological mechanisms.

\section{II.P}

Mitotic kinases, phosphatases and neural progenitor self-renewal

William Chia ${ }^{l}$, Hongyan Wang, Wayne G Somers, Kaichen Chang

${ }^{1}$ Temasek Life Sciences Laboratory, National University of Singapore
Defects in the asymmetric division of stem/progenitor cells can lead to an imbalance in the generation of self-renewing vs differentiating daughter cells, one of the early events which may lead to malignant transformation. Studies on Drosophila neural progenitors, neuroblasts, one of the better models for understanding asymmetric divisions, have revealed that several cell cycle regulators also play important roles in mediating neuroblast asymmetric divisions, and this connection may explain the tumour suppressor property of some of these molecules. In this talk I will provide:

1. an overview of our current knowledge of the machinery which control neuroblast asymmetric divisions

2. Summarise recent work from the lab on several molecules some of which are normally construed to be key cell cycle regulators and how they impinge on the machinery which control neuroblast asymmetry to mediate asymmetric protein localisation and/or the decision to self-renew or differentiate.

II. 2

The Drosophila PDZ Protein Canoe: A novel player during asymmetric cell division

Stephan Speicher ${ }^{1}$, Anja Fischer ${ }^{2}$, Juergen Knoblich ${ }^{2}$, Ana Carmena ${ }^{3}$

${ }^{1}$ Instituto de Neurociencias de Alicante-CSIC/UMH

2 IMBA, Vienna

${ }^{3}$ Instituto de Neurociencias de Alicante-CSIC/UMH

Asymmetric cell division is a conserved mechanism to generate cell diversity. Neuroblasts (NBs), the progenitors of the Drosophila central nervous system, undergo asymmetric cell divisions. In a stem cell-like fashion, polarized NBs divide to give rise to an apical NB, which keeps the stem cell-like properties, and a basal ganglion mother cell, committed to differentiate. We have analyzed the function of the PDZ protein Canoe (Cno) during asymmetric NBs division. Cno and its vertebrate counterpart AF-6 are cytoplasmic proteins normally associated to cellular junctions. We found that Cno colocalizes with the PDZ protein Bazooka/Par3 at the apical pole in metaphase NBs. cno loss of function mutants showed an altered distribution of the cell-fate determinants Numb, Prospero and Brat, as well as randomized orientation of the mitotic spindle in metaphase NBs. The unequal size of NBs daughter cells was also affected in cno mutants. Indeed, neuronal lineages were compromised in cno mutants. Epistatic interactions between Cno and other apical proteins placed Cno downstream of Inscuteable (Insc)Partner of Inscuteable (Pins)-G $\alpha \mathrm{i}$ and upstream of the NuMA-related protein Mushroom body defect. Further- 
more, co-immunoprecipitation assays showed that Cno is forming a complex with Pins in vivo. Failures in the asymmetric division of muscle and heart progenitors were also detected in cno mutants. Hence, our data reveal a function of Cno as a new player during asymmetric cell division.

\section{3}

\section{Using DamID to dissect the Transcriptional Networks determining Drosophila Neural Stem Cell Fate}

Tony Southall ${ }^{1}$, Andrea Brand ${ }^{1}$

${ }^{1}$ Gurdon Institute, University of Cambridge

Drosophila neural stem cells (neuroblasts) divide in an asymmetric self-renewing manner. At each division they produce another neuroblast and a ganglion mother cell (GMC), which divides only once more to give rise to 2 neurons and/or glial cells. Prospero is an atypical homeodomain transcription factor that is asymmetrically segregated from the neuroblast to the GMC. Previously we have shown that Prospero acts as a binary switch between self-renewal and differentiation. However, little is known about the transcriptional networks that Prospero operates within or against, in controlling neural stem cell maintenance and neuronal differentiation. To begin to dissect these transcriptional networks we have profiled, on a full genome scale, three additional transcription factors, Asense, Deadpan and Snail. The resulting networks being generated reveal a large overlap between these four transcription factors and are allowing us to identify novels players in neurogenesis. In order to further understand how Prospero is able to repress selfrenewal and promote differentiation, we have used bioinformatics tools to identify putative Prospero cofactor binding sites from our DamID data. Using a yeast one-hybrid system we have identified a factor that binds to one of these sites. Functional characterisation of this factor is underway. The mechanism of Prospero's action and the dissection of related gene regulatory networks will provide important insights into neural stem cell maintenance and neurogenesis.

II.4

Regulation of inter-segmental diversification of a neuroblast lineage in the embryonic CNS

Christian Berger ${ }^{1}$, Ramakrishnan Kannan ${ }^{2}$, L.S. Shashidhara $^{3}$, Gerd Technau ${ }^{4}$
${ }^{1}$ IMBA-Institut of Molecular Biotechnology Austria

${ }^{2}$ NINDS-National Institute of Neurological Disorders and Stroke

${ }^{3}$ CCMB-Centre for Cellular and Molcular Biology

${ }^{4}$ Institute of Genetics, University of Mainz, Germany

Upon gastrulation neural stem cells (neuroblasts, NBs) delaminate from the neuroectoderm in a stereotype spatial and temporal pattern, which is almost identical among thoracic and most of the abdominal segments. Specification and development of serially homologous NBs among these segments appear to be controlled by the same genetic mechanisms, as reflected by the expression of corresponding combinations of molecular markers and the production of very similar embryonic cell lineages. However, some of the serially homologous NBs generate lineages that differ significantly in thorax and abdomen. We have examined the events by which segment-specific differences are generated within a NB lineage in the embryonic CNS. NB6-4 generates both neuronal and glial cells in thoracic, but only glial cells in abdominal segments. We have recently shown that this difference is dependent on the function of $\mathrm{CycE}$. $\mathrm{CycE}$ is necessary and sufficient to generate neuronal cells in thoracic segments, where it is expressed exclusively in the neuronal part of the lineage. Loss of $\mathrm{CycE}$ leads to loss of neuronal cells in the thoracic lineage, whereas gain of $\mathrm{CycE}$ leads to de novo formation of neurons from the abdominal lineage. Intriguingly, all other regulators of cell cycle tested did not give any such phenotypes leading to the hypothesis that this function of $\mathrm{CycE}$ might be a new function possibly independent of its role in cell cycle progression. To investigate this hypothesis, we are following different approaches: a) testing the function of $\mathrm{CycE}$ in the background of string mutations, in which NBs are born but do not divide, b) generation of transgenic lines expressing various CycE-deletion constructs to test whether we can separate the function of the protein in regulating cell division and cell fate specification. Furthermore, we are testing the impact $\mathrm{CycE}$ might have on the asymmetric localization machinery, which is used to generate two different cell types from a common precursor. Preliminary results from these approaches will be presented.

II.6

From stem cell to unique neuron: Temporal transitions in an identified CNS progenitor cell by feedforward combinatorial coding

Magnus Baumgardt ${ }^{1}$, Daniel Karlsson ${ }^{1}$, Javier Terriente ${ }^{2}$, Fernando J. Díaz-Benjumea ${ }^{2}$, Stefan Thor ${ }^{1}$ 
${ }^{1}$ Dept. of Clinical and Experimental Medicine, Linköping University, Linköping, Sweden

${ }^{2}$ Centro de Biología Molecular, Universidad AutónomaCantoblanco, Madrid, Spain

It is well-established that progenitor cells in both the invertebrate and vertebrate CNS change their competence over time to generate different cell types at different time points. However, the mechanisms controlling these temporal changes are still poorly understood. In the Drosophila CNS the transcription factors $\mathrm{Hb}-\mathrm{Kr}-\mathrm{Pdm}-$ Cas-Grh act as a 'temporal gene cascade' to change progenitor competence over time in most if not all neuroblasts. However, there are several unresolved issues concerning this temporal gene cascade: 1) What are the downstream targets of the temporal genes? 2) How is the 'window size' of each temporal window controlled? 3) Given that several different types of cells can be generated within the same temporal window: How are the temporal windows sub-divided? To address these issues, we are focusing our studies on the neuroblast 5-6. We have identified several distinct neuronal cell types generated towards the end of this lineage, and, importantly, combinations of cell fate determinants that act to specify these cells. This allows us to address the connection between temporal genes, cell fate determinants and unique cell fates in a highly defined manner. We find that the latter part of the 5-6 lineage ends with a large Cas window that is sequentially sub-divided by feedforward/ feedback mechanisms into several sub-windows. Within each sub-window a unique set of postmitotic cell fate determinants is expressed which in turn dictate unique neuronal cell fate.

\section{II.7}

\section{Antagonistic roles for two $\mathrm{Hox}$ genes in regulating segment-specific survival of differentiated motoneur- ons in the embryonic central nervous system}

\author{
Ana Rogulja-Ortmann ${ }^{1}$, Gerd Technau ${ }^{1}$ \\ ${ }^{1}$ Institute of Genetics, University of Mainz
}

The generation of morphological diversity among segmental units of the nervous system is crucial for correct matching of neurons with their targets and formation of functional neuromuscular networks, but the mechanisms leading to segment diversity in the CNS are still largely uncovered. We are investigating the role of programmed cell death in this patterning process, and have found that the Hox genes Ultrabithorax (Ubx) and Antennapedia (Antp) regulate segment-specific survival of differentiated motoneurons in the ventral nerve cord of Drosophila embryos. We show that $U b x$ is required to activate segment-specific apoptosis in these cells, and that their survival depends on Antp. Expression of the Ubx protein is strongly upregulated in the doomed motoneurons shortly before they undergo apoptosis, and our results indicate that this late upregulation is required to activate cell death. We further demonstrate that $U b x$ executes this role by preventing Antp from promoting cell survival. Thus, two Hox genes contribute to segment patterning and diversity in the embryonic CNS by carrying out opposing roles in the survival of specific differentiated motoneurons.

II. 8

\section{Controlling neural stem cell fates in the Drosophila optic lobe}

Boris Egger ${ }^{1}$, Kathrina S. Gold ${ }^{1}$, Andrea H. Brand ${ }^{1}$

${ }^{1}$ Wellcome Trust/Cancer Research UK, Gurdon Institute, Dept. of Physiology, Development and Neuroscience, University of Cambridge

Stem cells undergo symmetric or asymmetric divisions to proliferate, self-renew and produce differentiating cell types. The correct balance between proliferation and differentiation is crucial since its misregulation can cause tumorous overgrowth. In the Drosophila optic lobe, symmetrically dividing neuroepithelial cells transform into asymmetrically dividing neuroblasts. In order to gain an insight into how neural stem cell fates are maintained and generate differentiating progeny during development we used microarrays to compare the genome-wide transcription profiles of optic lobe neuroepithelial cells and neuroblasts. Strikingly, several genes were upregulated in neuroepithelial cells, which encode Notch pathway components, suggesting that these genes may be involved in maintaining the neuroepithelial cell fate. Indeed we found that Notch loss-of-function mutant clones delaminate from the neuroepithelium at ectopic positions and prematurely differentiate. The microarray analysis also directed our focus to an apparent transition zone between optic lobe neuroepithlial cells and neuroblasts. In this transition zone we found the expression of the proneural gene lethal of scute (l'sc), which was shown to be required for the timely conversion of neuroepithelial cells to neuroblasts. We made a l'sc reporter line to visualize and isolate transitory neuroepithelial cells. This will enable us to carry out further microarray experiments to identify novel genes involved in regulating neural stem cell fate transitions. 
II. 10

Organizing Nervous System Architecture: Genetic Control of Drosophila Dendritic Arborisation Neuron Number, Position, and Identity

MD. Rezaul Karim ${ }^{l}$, W. Moore Adrian

1 Molecular Neuropathology Group, RIKEN Brain Science Institute

Formation of a complex nervous system involves the generation of a large diversity of neuron classes. To examine how such neuron diversity arises, we are investigating the control of Drosophila Dendritic Arborisation (DA) peripheral sensory neuron fate. Each individual DA neuron is derived from a single External Sensory Organ Precursor (ESOP) cell. During Drosophila embryogenesis ESOPs always form in stereotypic positions, and the position of ESOP formation prefigures the class of DA neuron to which it will give rise. As previously demonstrated in other systems, we find that DA neuron fate is determined by pre-patterning transcription factors expressed in the epithelium before neuronal precursor (ESOP) cell formation. The same factors required for pre-patterning DA neuron identity are also required for the maintenance of the embryonic segmental boundary signaling center that secretes Wingless and Hedgehog. Wingless and Hedgehog induce neurogenesis in the pre-patterned epithelium by switching on proneural gene expression; however they do not seem to play any role in controlling ESOP or subsequent DA neuron fate. Several different ESOP cells give rise to DA neurons of the same class. Surprisingly we find that these different cells arise in epithelial regions that are pre-patterned by different factors, expresses different proneural genes, and has different requirements for Hedgehog and Wingless signaling.

II. 11

\section{Apterous role in the regulation of corazonin expression}

Marta Andrés ${ }^{1}$, María Losada ${ }^{1}$, Isabel Molina ${ }^{1}$

${ }^{1}$ Departamento de Biología. Universidad Autónoma de Madrid

Corazonin is a neuropeptide from the central nervous system of insects with a greatly localized expression pattern.

Neuropeptides transcriptional regulation is known to be a complex process with both spatial and temporal variations. Recent studies indicate that this regulation is stablished by a combination of different types of transcription factors and extracellular signals which is unique for each neuropeptide. This combination could change even between different clusters of cells expressing the same neuropeptide. Apterous is a transcription factor from the LIM-HD family. It is known to act as a regulator in the expression of other neuropeptides like FMRFamide and leukoquinin. Our results show that Apterous is playing a role in the regulation of corazonin expression. Apterous does not colocalize to $\mathrm{Crz}$ in any of the corazoninergic cells. However, apterous mutants show ectopic expression of corazonin in the lateral protocerebrum and in the ventral ganglion. In these cells Apterous is likely to act as inhibitor of corazonin expression. However, we also report the presence of ectopic corazoninergic cells in individuals in which Apterous is overexpressed in the lateral protocerebrum. These results indicate that Apterous is a component of a combinatorial code that regulates corazonin expression. We will focus our further studies on trying to identificate other components of this combinatorial code

II.12

\section{Analysis of the specification of the Leucokininergic cell} fate

Jonathan Benito-Sipos ${ }^{1}$, Alicia Estacio ${ }^{2}$, Marta Moris ${ }^{2}$, Magnus Baumgardt ${ }^{3}$, Stefan Thor $^{3}$, Fernando J.DiazBenjumea $^{2}$

${ }^{1}$ Universidad Autónoma de Madrid

${ }^{2}$ CBM-CSIC

${ }^{3}$ University Of Linköping

The genetic and molecular mechanisms required for neuronal fate specification are poorly understood. In Drosophila CNS individual neurons only express one or a reduced number of the many identified neuropeptides. In this work we have analyzed the mechanisms underlying the specification of the Leucokinine (LK) expressing neurones. The larval leucokinergic system is formed for only four different types of neurons. We are focused our work in the ABLKs that form a group of 14 neurones located in the abdominal segments (A1-7). Very little it is known about the genes and the mechanisms involved in the specification of the LK fate. Here, by genetic analysis, we have identified several genes involved in the specification of ABLK fate. We also provide data that identify the neuroblast progenitor ABLK neurons.

II.13

sox-like regulates the levels of SoxN protein 1

Marita Buescher ${ }^{1}$, Wei Hao Chan ${ }^{2}$, William Chia ${ }^{1}$

${ }^{1}$ Temasek Lifesciences Laboratory

${ }^{2}$ Nanyang Polytechnic 
The HMG box transcription factor SoxNeuro (SoxN) plays a vital role in Drosophila development. Previous studies indicate that SoxN is essential for neuroblast formation, axon pathfinding and the development of the ventral denticles. SoxN RNA/protein expression is tightly regulated during embryogenesis. However, while aspects of the transcriptional regulation of SoxN have been examined, the regulation of SoxN protein levels has not been addressed as yet.

We have isolated three lines whose phenotypes strongly resemble that of SoxN mutants: we observed a severe loss of neuroblasts, axon pathfinding defects and impaired denticle formation. All three lines fail to complement each other but do complement mutant alleles of SoxN indicating that they represent alleles of a gene different from SoxN. We have named this gene "soxlike". Consistent with the mutant phenotype of the soxlike alleles, we found that SoxN protein is nearly absent at all stages of embryonic development. By contrast, SoxN RNA expression in sox-like embryos is similar to that observed in SoxN mutant embryos. These results suggest that sox-like is required for the regulation of SoxN protein levels. sox-like function appears to target specifically SoxN protein since the levels of Dichaete, a closely related HMG domain protein, are similar to those observed in SoxN mutant embryos. We have identified a deficiency which fails to complement sox-like and are currently sequencing appropriate candidate genes.

\section{II.14}

\section{Analysis of the mitosis-dependent posttranscrip tional regulation of seven-up activity in neuroblast lineages}

Sascha Eder ${ }^{1}$, Ulrike Mettler ${ }^{1}$, Joachim Urban ${ }^{1}$

${ }^{1}$ University of Mainz, Institute for genetics

Both in vertebrates and invertebrates, neural stem cells generate different cell types at different times during development. It has been suggested that this process depends on temporal identity transitions of neural progenitors, but the underlying mechanism has not been resolved yet. Recently, Drosophila neuroblasts (NBs) have been shown to be an excellent model system to investigate this subject. Here, changes in temporal identity are regulated by sequential and transient expression of transcription factors in the NB, such as Hunchback ( $\mathrm{Hb})$ and Krüppel $(\mathrm{Kr})$. The temporal expression profile is maintained in the progeny. $\mathrm{Hb}$ is expressed first and thus defines the earliest identity in a given lineage. Transition to $\mathrm{Kr}$ requires the termination of $\mathrm{hb}$ expression which occurs in response to mitosis, and is mediated by Seven-up (Svp). Recent results provided evidence that the dependency of Svp activity on mitosis is due to an inhibition of the nuclear export of svp mRNA and/or to a mitosisdependent activation of translation (Mettler et al., 2006). To investigate this mechanism we are currently testing whether the untranslated regions (UTRs) of the different svp splice variants are involved in this regulation. Our approach is to express constructs containing different svp UTR sequences fused to an eGFP open reading frame in mitosis-arrested neuroblasts of string mutant embryos. The results of these experiments will be presented. This work has been supported by the Deutsche Forschungsgemeinschaft (Ur42/6-1).

\section{II.15}

A Drosophila Runt family transcription factor, Lozenge, specifies the olfactory receptor neuron class in a dose-dependent manner

Keita Endo ${ }^{l}$, Kei Ito ${ }^{1}$

${ }^{1}$ Institute of Molecular and Cellular Biosciences, The University of Tokyo

In Drosophila, the olfactory receptor neurons (ORNs) are differentiated into $\sim 50$ classes, each of which expresses specific odorant receptors and innervates a specific glomerulus in the antennal lobe of the brain. To understand the molecular mechanisms underlying this neuronal differentiation, we have been studying the function of a Runt family transcription factor, Lozenge, in the ORN development. We first analyzed the axonal projection of ORN clones homozygous for a lozenge null allele, and found that the mutant clones projected only to a small subset of glomeruli. Then, we systematically changed the severity of the lozenge mutation using the temperaturesensitive alleles, and found that more ORN classes differentiated and projected normally as the level of Lozenge was gradually restored. Next, we examined whether different levels of Lozenge instruct differentiation of different ORN classes, by visualizing them in the antenna using the OR-Gal4 transgenes. To decrease and increase the level of Lozenge, we used the lozenge mutation and a chromosomal duplication of the wildtype lozenge locus, respectively. We found that ORNs of particular classes were lost when the Lozenge level was decreased, whereas their number increased when Lozenge was increased. ORNs of other classes that were unaffected by the decrease of Lozenge, on the contrary, decreased in number when Lozenge was increased. These data strongly suggest that Lozenge specifies the ORN class in a dosedependent manner. 
II.17

Functional analysis of hunchback protein domains in the context of temporal specification of drosophila neuroblasts

Sebastian Jansen ${ }^{1}$, Viktoria Linne ${ }^{1}$, Ulrike Mettler ${ }^{1}$, Joachim Urban ${ }^{1}$

${ }^{1}$ Department of Genetics

Neuroblasts (NBs) within the Drosophila ventral nerve cord produce specific cell lineages in which unique cell types are generated in a fixed temporal order. This birthorder dependent specification of NB progeny is controlled by a set of genes encoding transcription factors, which are sequentially and transiently expressed within the NBs, and whose expression is inherited by the ganglion mother cell (GMC) born at that time. The earliest of these factors is the zinc finger protein Hunchback $(\mathrm{Hb})$. We and others have already shown, that continuous expression of $\mathrm{Hb}$ beyond its normal time window arrests the NB in a "young" state leading to the production of supernumary cells with "early" cell fates (Isshiki et al., 2001, Novotny et al., 2002). Additionally, there is evidence that this factor is also involved in regulating the width of a competence window in which $\mathrm{Hb}$ itself but also later temporal specification factors can act (Cleary and Doe, 2006). To get an insight into how $\mathrm{Hb}$ might exert these functions, we are investigating which of the evolutionary conserved domains of this protein are functionally needed in this context by overexpressing mutated versions of $\mathrm{Hb}$ during NB lineage development. So far we could show that the "D-box" is absolutely essential for the specification of the "early" neural cell fate, while the A-box and the terminal zinc fingers seem not to be required in the investigated lineages. This work is supported by the Deutsche Forschungsgemeinschaft (Ur42/6-1).

\section{21}

IRM-proteins in wing development: Involvement in the regular spacing of sensory bristles in the anterior wing margin

Gerit Arne Linneweber ${ }^{1}$, Karl-Friedrich Fischbach ${ }^{1}$

${ }^{1}$ Faculty of Biology, Albert-Ludwigs-University Freiburg

The Neph-like proteins IrreC/Rst, Kirre and the Nephrinlike proteins Hbs, SNS cooperate in the interaction of different polarized cell types. Here we describe their concerted action in the development of sensory organs at the anterior wing margin and add to the justification to give this protein ensemble a common name, the Irre Cell Recognition Module (IRM).

The anterior wing margin consists of an array of 3 types of bristles (slender, recurved and stout), that are arranged in a regular pattern. We show the involvement of all 4 IRM proteins in the development of this array. To achieve their function the proteins show differential gene expression and a well defined apical-basal polarity.

Using gain of function, RNAi and mutant analysis, we found out that the IRM is essential for the regular spaced pattern of the bristles. This is especially interesting as the spacing pattern between the recurved bristles is about 3-4 cell diameters in adults, which is difficult to explain via lateral contact inhibition. The IRM proteins stabilize via heterophilic interaction the sensory organ precursors (SOPs) at distinct positions and determine the number of cells between them. Disruption of the expression pattern of the IRM proteins, however, ranges from a phenotype, where the number of bristles is altered, to phenotypes where only the spacing pattern of bristles is disrupted, suggesting a role of IRM proteins in the differentiation of SOPs as well.

\section{22}

\section{Developmental origin of a motor control centre in Drosophila}

Zoe Ludlow $^{1}$, Katherine E White ${ }^{1}$, Frank Hirth ${ }^{1}$

${ }^{1}$ MRC Centre for Neurodegeneration Research, Institute of Psychiatry, King's College London

The Drosophila central complex (cc) has been described as a centre for the higher control of locomotion. It comprises four neuropils: the ellipsoid body (eb), fanshaped body, paired noduli and protocerebral bridge. The eb is involved in flight control, spatial memory and walking behaviour. Cells contributing to the eb can be visualised using enhancer-trap cc-Gal4 lines, and have been traced back to early pupal stages. In this study, we have identified a tool to locate and trace eb neurons. This tracer line shows expression in two cell clusters in each hemisphere of the adult brain, one of which projects via the lateral triangles to the eb; these cells include ring neurons R1-R4. The tracer line has been used to track these cells back in development and marks two clusters of cells that are already present in the embryonic brain, where they coincide with neuromere boundaries. Using en-Gal4, UAS- $m C D 8:: G F P$ and other markers, we have been able to identify the neuroblasts from which these two neuronal clusters derive. In a search for factors involved in the development and specification of these eb precursor neurons, we have identified heartless, an Fgf8 receptor homologue. Our study of Fgf8 mutants indicates that this 
signalling pathway is involved in the maintenance of the embryonic eb precursor neurons. RNAi knockdown experiments as well as cell ablation and inactivation experiments are under way in order to determine the functional anatomy of these cells.

The tiny Hairless protein from Apis mellifera behaves like a bona fide Notch antagonist in Drosophila melanogaster

Dieter Maier ${ }^{1}$, Anna Chen ${ }^{1}$, Anette Preiss ${ }^{1}$, Manuela Ketelhut $^{1}$

${ }^{1}$ Institut für Genetik (240), Universität Hohenheim

Hairless acts as a general antagonist of Notch signal transduction in Drosophila. By binding to $\mathrm{Su}(\mathrm{H})$ and corepressors Groucho (Gro) and $\mathrm{CtBP}$ it assembles a repression complex on Notch target genes. We have cloned the Hairless orthologue from honeybee Apis mellifera $(\mathrm{AmH})$. We find that $\mathrm{AmH}$ is only a third of the size of Drosophila melanogaster Hairless protein $(\mathrm{DmH})$. However, it retains the known structural elements, a $\mathrm{Su}(\mathrm{H})-$, a Gro- and a CtBP-binding domain. Indeed, the AmH protein physically interacts with all three Drosophila proteins. Hence, despite an evolutionary distance of more than 250 millions years and a striking divergence, AmH has retained the biochemical properties of $\mathrm{DmH}$ in this assay. Next, we addressed the question, whether the small AmH protein retains its function in Drosophila. Several fly lines were generated that allow overexpression of wild type and mutant $\mathrm{AmH}$ proteins. This affects bristle, eye and wing development as expected for a bona fide Notch antagonist. Accordingly, AmH represses the Notch target gene vestigial in the wing disc like DmH. Moreover, AmH rescues Hairless mutant phenotypes, indicating that it can largely replace $\mathrm{DmH}$. Protein variants with mutant binding sites for Gro, CtBP or both showed the expected diminished activity in the fly. Overall, our findings show that the Hairless orthologue from Apis mellifera has retained most of the function of its Drosophila counterpart despite a considerable degree of divergence.

II. 24

Neuroepithelial stem cells are converted into neural progenitors during an elongated interphase in drosophila larval optic lobe

Minako Orihara-Ono ${ }^{1}$, Keiko $\mathrm{Nakao}^{2}$, Hideyuki Okano ${ }^{3}$

${ }^{1}$ Dept. Physiology, Keio Univ., Sch. Med.
${ }^{2}$ Dept Physiol, Saitama Med Univ

${ }^{3}$ Dept. Physiology, Keio Univ. Sch. Med.

Although symmetrically proliferating neuroepithelial stem cells (NESCs) give rise to asymmetrically dividing neural progenitors (NPs), little is known about this change/ transition/emergence. Here we show that NESCs of the outer proliferating centre (OPC) of the Drosophila larval optic lobe were converted into NPs while they are passing through an elongated interphase to produce nascent NPs. Thus this period linked an exponentially amplifying cycle of NESCs with an asymmetric division typical of NPs. During this specific transition the cells directed their course toward differentiation, altered cell cycle progression pattern, lost their strict adhesion and left from symmetric proliferation seen in undifferentiated stem cells. Once they were completed the transition, the cell cycle progression pattern changed again to divide more rapidly ever since.

II. 25

Dorsoventral patterning in the developing brain of Drosophila

Janina Seibert ${ }^{1}$, Dagmar Volland ${ }^{1}$, Gerhard M. Technau ${ }^{1}$, Rolf Urbach ${ }^{1}$

${ }^{1}$ Institute of Genetics, University of Mainz

In the developing brain expression of these genes is more complex (compared to the VNC) and significantly differs among the brain neuromeres. These differences and the fact that large parts of the pNE and more than half of the brain NBs express neither columnar gene, suggest the existence of additional factors which control dorsoventral patterning of the early brain. We show that SoxN, Dichaete (D) and Drosophila Egf receptor (Egfr) are expressed in a highly dynamic pattern in major parts of the pNE and in specific sets of NBs of all three brain neuromeres, whereas $N k 6$ is confined to the ventral/ intermediate regions of the Tc and Dc. Mutant analysis indicates that $N k 6$ is positively regulated by $v n d$ in the posterior head segments, whereas Egfr seems to have no direct influence on $N k 6$ expression. Furthermore, Egfr seems to be necessary to activate $D$ expression in the dorsal and intermediate regions of Tc, Dc, and Pc but is dispensable for activation of $D$ in other areas of the brain.

II. 26

Drosophila Olfactory Local Interneurons and Lateral Projection Neurons arise from a Single Lineage.

Sonia Sen ${ }^{1}$, Abhijit Das ${ }^{2}$, Robert Lichtneckert ${ }^{3}$, Kei Ito $^{4}$, Veronica Rodrigues ${ }^{1}$, Heinrich Reichert ${ }^{3}$ 
${ }^{1}$ Genetics and Development, National Centre for Biological Sciences.

${ }_{2}$ Department of Biological Sciences, Tata Institute of Fundamental Research.

${ }^{3}$ Biozentrum, University of Basel.

${ }^{4}$ Institute of Molecular and Cellular Biosciences, University of Tokyo.

Encoding of information about olfactory stimuli in Drosophila melanogaster occurs in the antennal lobe by activities of the projection and local interneurons. Several studies have addressed the mechanisms of specification and development of the olfactory receptor neurons and projection neurons. In this study we present our analysis of the development of the local interneurons that innervate the antennal lobe. We have carried out MARCM and dual expression-control MARCM experiments to trace the $\sim 60$ local interneurons that contribute to the adult lobe. We find that the lateral neuroblast of the deutocerebrum divides to generate local interneurons as well as $\sim 40$ projection neurons. The early divisions of this neuroblast gives rise to only local interneurons while later divisions produce both cell types in an atypical manner. We have used antibody markers coupled with genetic marking strategies to describe this lineage map. Finally we confirm and extend previous data demonstrating that the lateral neuroblast requires the normal function of the homeodomain containing the gap gene empty spiracles.

\section{27}

Identification of a novel tumor suppressor in Drosophila larval brain neuroblasts

\section{Cheng Wang $^{1}$, Harpreet Sidhu ${ }^{1}$, Hongyan Wang ${ }^{1}$ \\ ${ }^{1}$ DUKE-NUS Graduate Medical School, Singapore}

Drosophila neuroblasts (NBs), neural stem cells, undergo asymmetric divisions to maintain delicate balance between self-renewing and differentiation. The loss of proliferation control of neuroblasts may lead to tumor formation. Drosophila larval brain neuroblast has recently emerged as a novel model for the study of stem cell selfrenewal and tumourigenesis. However, the mechanisms underlying the growth control of neural stem cells remain elusive. To uncover novel molecular mechanisms, we aim to identify and characterize of novel tumor suppressor genes in the larval brains. In a recent genetic screen, we have isolated a novel tumor suppressor mutant that showed neuroblast overproliferation phenotypes in the larval brains. This novel tumor suppressor gene inhibits excess neural stem cell self-renewal and promotes neuronal differentiation. We found that the brain tumor formation in this mutant is due to defects in asymmetric cell division of neuroblasts. Currently, we are conducting detailed characterization of this mutant.

\section{Fizzy-related/Cdh1 controls glial cell migration in the} embryonic PNS

Marion Silies $^{l}$, Christian Klämbt ${ }^{1}$

${ }^{1}$ University of Münster, Institute for Neurobiology

\section{Fzr/Cdh1 controls glial cell migration in the emryonic} PNS

\section{Marion Silies and Christian Klämbt}

University of Münster, Institute of Neurobiology, Badestr. 9, 48149 Muenster, Germany

A hallmark of most glial cell types is their pronounced motility. The relatively low number of glial cells in the fly nervous system facilitates the functional dissection of their migration and further differentiation.

We screened for mutants that have defects in the migration pattern of the peripheral glial cells and identified several alleles of the cell cycle regulator fizzy-related (fzr). During cell cycle, Fzr activates the E3 ubiquitin ligase complex APC/C and confers its substrate specificity.

Here, we demonstrate a new postmitotic function. Analysis of the $f_{z r}$ mutant phenotype suggests that glial migration is initially repressed by neuronal molecules. Cell type specific rescue experiments as well as further genetic analyses show that Fzr is required in motoraxons to non-autonomously control glial migration independent of the glial number. This leads to the question of what the relevant neuronal APC/C-Fzr target is. To identify such a substrate, we are currently combining computational with genetic methods to screen for new molecules regulated by APC/C-Fzr. We will present the analysis of a new substrate candidate.

Taken together, our data suggest a model in which first, motor neurons prevent the premature migration of glial cells and later, this restriction is overcome in a Fzrdependent manner.

III.4

Gcm induces neuroblast reprogramming and chromatin changes via CBP

Angela Giangrande ${ }^{l}$, Nagy Istvan $^{1}$, Erkosar Berra ${ }^{1}$, Komonyi Orban ${ }^{1}$

${ }^{1}$ Dept Cell and Dev Biology, IGBMC-CNRS, ULP 
Neural development relies on a complex regulatory network that controls the acquisition of neuronal and glial fates starting from neural stem cells, called neuroblasts in Drosophila melanogaster. The transcription factor $\mathrm{Gcm}$ is necessary for glial differentiation and is sufficient to induce ectopic gliogenesis in fly embryos. We here show that $\mathrm{Gcm}$ misexpression triggers neuroblast reprogramming. Interestingly, wild type neurons and glia display distinct chromatin organization and $\mathrm{Gcm}$ dependent neuron to glia transformation is accompanied by global gene expression as well as chromatin changes. Using pure embryonic cell populations, we demonstrate that $\mathrm{Gcm}$ recruits coactivator $\mathrm{CBP}$ to a target promoter and triggers histone $\mathrm{H} 3$ tail acetylation. Importantly, the fate transformation induced by $\mathrm{Gcm}$ requires such chromatin changes. These data show for the first time that a single transcription factor is able in vivo to reprogram multipotent precursors of the nervous system by triggering chromatin reorganization and subsequent gene expression changes

\section{III.5}

Receptor-type specification of Drosophila olfactory neurons from a pluripotent precursor is mediated through the chromatin regulator Psc.

Özkan Aydemir ${ }^{1}$, Georg Steffes ${ }^{2}$, Britta Kuhlmann ${ }^{1}$, Thomas Hummel ${ }^{1}$

${ }^{1}$ Institute of Neurobiology, University of Muenster

${ }^{2}$ Wellcome Trust Genome Campus, Hinxton, Cambridge

Sensory neurons in the olfactory system display a tight coordinated differentiation of receptor type expression and target neuron selection. In contrast to the mouse olfactory system, where the odorant receptor itself is involved in the control of both, single OR gene expression and single glomerulus targeting, the Drosophila ORs are dispensable for receptor and target cell selection. Here we show that the coordination of sensory and synaptic differentiation is mediated through the chromatin regulators Posterior Sex Combs (Psc). Loss of Psc leads to a mixing of the sensory and synaptic characteristic among the three ORNs housed in the at 4 pheromone-sensitive sensillum, without changing the overall at 4 number and distribution. Temporally-restricted Psc loss-of-function and rescue experiments show that Psc functions after initial at4 ORN specification. Surprisingly, ORN diversification into different sensory specifities is achieved through a dose-dependent Psc activity. As we did not observe defects in ORN differentiation for mutants affecting other components of the chromatin silencing complex, we are proposing a novel dynamic function of Psc in the control of neuronal target gene expression.
These data show that the coordinated differentiation of sensory neurons in the Drosophila olfactory system is mediated through the lineage-specific activity of a global gene regulator.

III.6

Acj6 acts both positively and negatively to regulate odor receptor gene choice in Drosophila

Lei Bai ${ }^{1}$, Aaron Goldman ${ }^{1}$, John Carlson ${ }^{1}$

${ }^{1}$ MCDB, Yale University

Little is known about how individual olfactory receptor neurons (ORNs) select, from among many odor receptor genes, which genes to express. The process is critical to the establishment of ORN populations that encode olfactory information. Acj6 (Abnormal chemosensory jump 6) is a POU-domain transcription factor essential for the specification of ORN identity and $\mathrm{Or}$ (odor receptor) gene expression in the Drosophila maxillary palp, one of the two olfactory organs of the fly; however, its mechanism of action in this process is unknown. Here we systematically examine the role of Acj6 in the maxillary palp and in a major class of antennal ORNs. We define an Acj6 binding site by a reiterative in vitro selection process, find that $O r$ genes regulated by Acj6 contain this site, and show by mutational analysis that these sites are required in vivo for normal $O r$ expression. We find that a novel ORN class in acj6 adults arises from ectopic expression of a larval Or gene. This larval gene is repressed by Acj6 in wild-type via an Acj6 binding site. A binding-site-swap experiment shows that the negative mode of regulation mediated by this particular Acj6 site is a function not of its sequence but of its context. Taken together, our results provide evidence that Acj6 acts directly in the process of receptor gene choice, that it plays a dual role, positive and negative, in the logic of the process, and that it acts in the partitioning of the larval and adult receptor repertoires.

The transmembrane receptor Golden goal guides $\mathbf{R 8}$ axons into the correct column and layer of the brain

Tatiana Tomasi ${ }^{1}$, Satoko Hakeda-Suzuki ${ }^{1}$, Si-Hong Luu ${ }^{1}$, Sandra Berger ${ }^{1}$, Stephan Ohler ${ }^{1}$, Takashi Suzuki ${ }^{1}$

${ }^{1}$ Max Planck Institute of Neurobiology

To sense the outside world, sensory neurons connect their axons to the central nervous system. In the target area of axons, neuronal processes often form columnar and 
layered structures. These structures are thought to underlie the functional substrate, since the modular and repetitive structure accounts for the specific connection between inputs and outputs. How are these columns/ layers formed during development is largely unknown. Using the Drosophila visual system as a model system, we could show that the transmembrane protein Gogo is required for the photoreceptor $(\mathrm{R})$ axon to enter the correct column in the target area of the brain. From the Gain and loss of function experiments, we suggest that gogo promotes axon-target recognition at the surface of the medulla to enable R8 axons to recognize their correct columns. From detailed structure-function experiments, we propose that gogo functions as a receptor that binds an unidentified ligand through its conserved extracellular domain. We also show that conserved tyrosines in the cytoplasmic domain of Gogo have to be dephosphorylated for the Gogo function. We also show genetic interactions with tyrosine phosphatases. We propose that the regulation of phosphorylation of Gogo protein is a crucial mechanism in guiding the $\mathrm{R} 8$ axon to the correct column and the layer. We anticipate that these findings will lead to the understanding of how the columnar and layered structure in the brain is formed during development.

\section{III.8}

\section{Plasticity of terminally differentiated neurons by switching rhodopsin expression}

Simon Sprecher ${ }^{1}$, Claude Des ${ }^{1}$

${ }^{1}$ Departement of Biology, New York University

Functional specificity of sensory neurons is achieved by the restricted expression of one given sensory receptor gene per sensory neuron type. In Photoreceptors (PRs) functional specificity is mediated through the expression of Rhodopsins. In addition to the PRs of the compound eye, the fly also receives light dependent input from a small group of PRs termed "eyelet". The eyelet is derived from the PRs of the larval eye, which is composed of about 12 PRs, 4 expressing the blue-sensitive (Rh5), the remaining 8 are green-sensitive (Rh6). We find that, during metamorphosis, all Rh6-PRs larval PRs die, while the Rh5-PRs switch fate to express Rh6. We further show that Ecdysone receptor functions autonomously both for the death of Rh6-expressing larval PRs and for the sensory switch of Rh5-PRs. Surprisingly, we find that genetic program underlying sensory respecification requires novel mechanisms and does neither depend on the players required for $\mathrm{Rh} 5 / \mathrm{Rh} 6$ regulation in the adult R8cell, nor for the larval PR-subtypes. We also show that the transcription factor Senseless mediates the very different cellular behaviors of Rh5- and Rh6-PRs. Senseless is restricted to Rh5-PRs and must be excluded from Rh6PRs to allow them to die at metamorphosis. This is a previously unidentified example of sensory respecification of terminally differentiated neurons.

Role of Poxn in Drosophila ellipsoid body development Shilpi Minocha ${ }^{1}$, Werner Boll ${ }^{1}$, Markus Noll ${ }^{1}$

${ }^{1}$ Institute of Molecular Biology, University of Zürich

Pox neuro (Poxn), a member of the Drosophila Pax gene family, encodes a transcription factor with a DNAbinding paired domain. It is expressed in discrete domains throughout brain development. A large fraction of the cells acquire a Poxn fate during larval development but are already present in the embryonic brain, as there is little mitotic activity in the Poxn expression domain during larval life. All cells in these Poxn expression domains represent immature neurons. In wild-type brains, the projection patterns of the larval and pupal Poxn-expressing neurons appear to represent a pattern analogous to that of the adult Poxn-expressing neurons, as antennal lobes, mushroom bodies, and lateral regions seem to be already targeted. The neurites from the Poxn-expressing cells form the ellipsoid body (eb) starting from late third instar. The dorsal cluster seems to contribute mainly to the eb formation. The eb forms a doughnut-shaped structure by $36 \mathrm{~h}$ after puparium formation (APF) and eventually forms a structure similar to the adult eb by $72 \mathrm{~h}$ APF. In Poxn mutant larvae, the neuronal projection pattern is symmetric but neurites seem to stall before turning towards the midline and lateral regions. In Poxn pupae, the development of the eb seems to be severely affected. Lineage analysis suggests that the same Poxn-expressing immature neurons seen in third-instar wandering larvae survive metamorphosis and eventually contribute to the formation of a functional eb in the adult brain.

Homologous nuclear receptors $f t z-f 1$ and $H r 39$ have opposite role on ecdysone receptor expression during Drosophila mushroom body $\gamma$ axon pruning.

Jean-Maurice Dura ${ }^{1}$, Ana Boulanger ${ }^{1}$, Chris Clouet $^{1}$, Thomas Guignard ${ }^{1}$, Adrien Flandre ${ }^{1}$

1 Department of Genetics\& Development, Institute of Human Genetics 
Developmental axon pruning is a general mechanism required in order to transform an immature neural circuit into a mature one. During Drosophila metamorphosis, mushroom bodies (MB) larva-specific dendrites and axons of early $\gamma$ neurons are pruned and replaced by adult-specific processes. We show here that the nuclear receptor $f t z-f l$ is required for this pruning. Contrarily to the expectations it is FTZ-F1 which is regulating EcRBI activity and not the way around. Moreover, $f t z-f l$ has two roles: one is to activate $E c R B 1$ expression, whose activity is compulsory for normal $\gamma$ remodelling. The second one is to repress expression of $\mathrm{Hr} 39$, the $f t z-f l$ homologous gene. If HR39 is inappropriately (ectopically) expressed in the $\gamma$ neurons it will block normal pruning by preventing EcRBI expression likely by competing the activation by endogenous FTZ-F1. It is the first indication that in vivo competition takes place between these two proteins. It was previously established that EcRBI is a target of the TGF- $\beta$ signalling pathway. We also show that the $f t z-f l / H r 39$ pathway is apparently independent of the TGF- $\beta$ signalling and that, therefore, two molecular pathways are acting independently on $E c R B 1$ expression during $\gamma$ neuron remodelling. It is not known how cell type-specific responses are achieved by the TGF- $\beta$ pathway. A second, independent pathway might provide such specificity.

\section{III.11}

IRM-proteins in neural development I: IrreC/Rst and Kirre expressing cell types in the pupal optic lobe of $D$. melanogaster

Till Felix Malte Andlauer ${ }^{1}$, Kokil Chaudhary ${ }^{1}$, KarlFriedrich Fischbach ${ }^{l}$

${ }^{1}$ Faculty of Biology, Institute of Biology III, AlbertLudwigs-University Freiburg

The immunoglobulin domain containing Neph-like cell adhesion molecules Irregular chiasm C/Roughest (IrreC/ Rst), Kin-of-Irre (Kirre) and their Nephrin-like ligands Sticks-and-Stones (SNS) and Hibris (Hbs) constitute the multipurpose "Irre Cell Recognition Module (IRM)" in Drosophila, which functions during muscle fusion, cell sorting in imaginal discs, axonal pathfinding and during target recognition. Stunning is the similarity of the immunoreactivity pattern of all four proteins in the pupal optic lobe after completion of axonal pathfinding at stages where sorting of the correct axonal and dendritic membranes, i. e. target recognition, is occuring. This immunoreactivity is specific for the neuropil regions and displays a pronounced stratification. In order to identify the underlying cell types we have screened several Gal4insertions in the irreC/rst and kirre regulatory regions that mimic the irreC/rst or kirre expression pattern. Using MARCM we identified a large number of cell types expressing these genes. MARCM on the basis of cell type specific Gal4-lines can now be applied in a next step to experimentally study the transport and function of these proteins in retinula cells as well as in visual interneurons and glial cells.

III.12

Amplifying brain neuroblasts contribute to central complex development in Drosophila melanogaster

Jasmin Balmer ${ }^{1}$, Robert Lichtneckert ${ }^{1}$, Bruno Bello ${ }^{1}$, Heinrich Reichert ${ }^{1}$

${ }^{1}$ Biozentrum University of Basel

Recently the existence of remarkably large neuroblast lineages consisting of approximately 500 neural cells has been discovered in the developing brain of Drosophila. These large lineages are produced by a specific set of neuroblasts that amplify proliferation through generation of intermediate precursors. Here we show that these neuroblasts generate progeny which contribute to the formation of the central complex. Using a specific Gal4 line, which labels the developing columnar cells of the central complex, together with molecular markers that identify the amplifying neuroblast lineages, we follow the developmental differentiation of the neurons generated by 4 pairs of these neuroblasts. Moreover, we carry out MARCM-based clonal analysis to characterize the morphological features of the progeny generated by each of the contributing neuroblasts in more detail. The novel mode of neurogenesis that gives rise to these central complex neurons ensures that a small number of neuroblasts can generate the large number of columnar cells that make up the Drosophila central complex

Composition and Specification of the NB6-4 lineage in the gnathal CNS neuromeres

Henrike Becker ${ }^{l}$, Christian Berger ${ }^{2}$, L.S. Shashidhara ${ }^{3}$, Gerhard, M. Technau ${ }^{1}$

${ }^{1}$ Institut für Genetik, Johannes Gutenberg Universität Mainz

${ }^{2}$ Institut of Molecular Biotechnical Biologie Austria Wien

${ }^{3}$ Indian Institute of Science Education and Research, Pune, India 
Segmental identities of neural precursor cells (neuroblasts, NBs) in the ventral nerve cord are conveyed by homeotic genes. In NB6-4 the specification of its thoracic versus abdominal identity depends on genes of the Bithorax Complex. Thoracic NB6-4 generates glial cells and neuronal cells. Since it requires no homeotic gene function it represents the ground state. The abdominal variant is under control of $a b d-A$ and $A b d-B$. These genes exert their function by repressing the cell cycle gene $C y c E$, which is necessary for generating the neuronal sublineage. Here we have investigated the composition and specification of serial homologs of NB6-4 lineage in the gnathal segments, which are under influence of genes of the Antennapedia-Complex $(A N T P-C)$. We could identify segment specific variants of NB6-4 lineages in the labial and maxillary segment. The labial NB6-4 generates three glia cells and five to six neuronal cells and thus is similar to the thoracic lineage. Maxillary NB64 generates four glial cells which resembles the abdominal variant. The mandibular NB6-4 homolog delaminates but could not be further investigated. All progeny of maxillary NB6-4 express $D f d$ and $S c r$, whereas labial NB6-4 cells express Antp. With help of loss- and gain-offunction experiments we revealed that $D f d$ plays the major role in mediating maxillary identity of NB6-4. Furthermore we found that $D f d$ is able to transcriptionally repress $c y c E$ similar to $a b d A$ and $A b d B$ function in abdominal NB6-4.

III.14

Connecting embryonic and postembryonic cell lineages in the Central Nervous System of Drosophila melanogaster

Oliver Birkholz ${ }^{1}$, Christian Berger ${ }^{2}$, Georg Vogler ${ }^{3}$, Gerhard M. Technau ${ }^{1}$

1 Institute of Genetics, Johannes Gutenberg-University, Mainz

2 Institute of Molecular Biotechnology of the Austrian Academy of Sciences, Vienna

${ }^{3}$ Burnham Institute for Medical Research, La Jolla

The relationship between embryonic and postembryonic cell lineages in the Central Nervous System (CNS) of Drosophila melanogaster is still unsolved. On the one hand the embryonic neuroblasts and their cell lineages have been characterized by marker gene expression (Doe, 1992 and Broadus and Doe, 1995) and DiI-labeling (Schmidt et al., 1997 and Bossing and Technau, 1994). On the other hand proliferation behaviour of postembryonic neuroblasts has been examined by BrDU-labeling (Taylor and Truman, 1992), and neural cell lineages in the larval thorax have been investigated by MARCM-techni- que (Truman et al, 2004). Unfortunately, DiI-labeling does not work in the larva and MARCM-technique is not practicable in the embryo, because of the long perdurance of the Gal 80 repressor. We are trying to establish a link between embryonic and postembryonic CNS lineages by two different approaches: The first approach combines a UAS-strain containing a flipout cassette $\left(\right.$ FLP $^{122}$; UAS FRT CD2 FRT CD8::GFP) with several Gal4-lines, whose expression we are checking in the embryo and in the larva. We will trace single cell lineages from embryonic to larval stages and try to identify these lineages in vivo. The second approach is aimed at establishing an embryonic mosaic analysis, and will be based on the substitution of the Gal 80 repressor by a fluorescence marker.

\section{III.15}

Analysis of the gustatory receptor mediated signaling cascade in Drosophila melanogaster

Nico Bredendiek ${ }^{1}$, Günter Gisselmann ${ }^{1}$, Hanns Hatt ${ }^{1}$, Eva M. Neuhaus ${ }^{1}$

${ }^{1}$ Dept of cell physiology, Ruhr University, Bochum

Drosophila melanogaster has approximately 60 gustatory receptor (GR) genes, which are expressed in the proboscis, first legs and wings, as well as in the female genitalia. Each gustatory receptor neuron expresses a subset of these GR genes. GRs are seven transmembrane proteins, which usually couple to G-protein linked signalling cascades. The aim of our study is to unravel the intracellular signaling cascade in the gustatory neurons, with special emphasis to the role of heterotrimeric Gproteins. Two of these receptors, Gr5a and Gr66a, have well understood response profiles to the tastants trehalose and caffeine. We investigated the involvement of different $\mathrm{G} \alpha$-subunits in sugar taste transduction in behavioural assays with transgenic flies expressing mutated G-proteins and RNAi directed against different G-proteins. We also analysed the expression of $\mathrm{G} \alpha$-subunits in the proboscis by quantitative RT-PCR and immunohistochemistry. The physiological properties of gustatory sensory neurons in response to sugar stimuli were then measured applying the tip recording technique.

III.16

IRM-proteins in neural development III: A putative role in layer selection and tiling

Kokil Chaudhary ${ }^{1}$, K.F Fischbach ${ }^{1}$

${ }^{1}$ Institute of Biology III, University of Freiburg 
Objective: The columnar and stratified organisation of the optic lobe facilitates specific contacts between peripheral input neurons and their postsynaptic targets. It is intriguing that the IRM-proteins IrreC/Rst, Kirre and their ligands SNS and Hbs, which are known to be involved in cell sorting in the eye imaginal disc, are expressed in the neuropil layers of the optic lobes, where the presumptive synaptic partners have to contact each other. In the present study, we are trying to elucidate IRM function in the maturing neuropiles by focusing on the projections of R7/R8 neurons into the distal medulla.

Methods: Using the Gal4/UAS system, we study the gain of function as well as RNAi mediated reduction effects of wild type and mutant protein levels in R7 terminals as well as in their targets. We also perform clonal analysis using the conventional FRT-recombinase method and the MARCM method in elucidating cell autonomous functions.

Results and conclusions: Targeted modulation of IrreC/ Rst and Kirre protein levels using panR7-Gal4 interferes with the shape of R7 terminal, especially tiling at M6 is affected. IrreC/Rst and Kirre overexpression lead to a dramatic increase in filopodia at midpupal stages. In the target region, knock down of Kirre leads to layer selection defects of R7 terminals and overexpression of $\mathrm{Hbs}$ to tiling defects.

\section{III.17}

\section{Identification of new genes regulating neuroblast divisions}

Louise Cheng ${ }^{1}$, Cedric Maurange ${ }^{1}$, Jennifer Grant $^{1}$, Julia Pendred $^{1}$, Alex P Gould ${ }^{1}$

1 Developmental Neurobiology, National institute for medical research

We are interested in the mechanisms that regulate the final size and shape of the developing CNS. We have recently completed pupal-lethal and MARCM screens of chromosome III to identify mutations affecting CNS growth. From 4,600 EMS mutagenised chromosomes, we recovered 177 lethal complementation groups showing altered spatiotemporal patterns of neuroblast divisions. Some of the corresponding genes have been mapped to transcriptional components such as the mediator, integrator and various chromatin modifying complexes. In addition, we recovered atypical undergrowth alleles of the tumour suppressors Scribble and $\mathrm{E}(\mathrm{spl})$. We will present a phenotypic analysis of the genes isolated in the screen and discuss how this may provide new insights into the mechanisms terminating neuroblast divisions and thus limiting the final size of the developing CNS.
III.18

\section{Screening for potential regulators of glial development in the Drosophila visual system}

Carole Chotard ${ }^{1}$, Iris Salecker ${ }^{1}$

${ }^{1}$ Division of Molecular Neurobiology, National Institute for Medical Research, London NW7 1AA, U.K.

The Drosophila visual system is composed of 8 photoreceptor cell types (R-cells). R1-R6 axons project from the eye to the optic lobe, terminating between two rows of glia in the lamina. These glia play an important role in mediating R-cell axon targeting.

To identify novel factors controlling R-cell axon targeting and glial development in the optic lobe, we have performed a mutagenesis screen using a FLP/FRT system-based genetic approach, generating somatic clones in lamina neurons and glia in the optic lobe while keeping the eye wild type.

We have isolated 50 mutant candidates with strong R-cell projection defects and uncovered 2 alleles of the chronologically inappropriate morphogenesis gene (chinmo) after mapping. Chinmo encodes a BTB-zinc finger transcription factor. Phenotypic analysis of chinmo mutants revealed severe defects in R-cell axon targeting and glia in the lamina, suggesting a specific role for chinmo in glial development.

We have also designed a P-element Gal4 insertion screen to identify new markers for neurons and glia within the optic lobe. One of the 1100 lines established showed specific reporter gene expression in lamina glia at larval and adult stages. The P-element is inserted in the Socs36e gene encoding a Suppressor of Cytokine Signaling protein, known to be a negative regulator of the JAK/STAT pathway. We are currently generating Socs36e mutant alleles to gain insight into the potential role of Socs36E in mediating optic lobe glial development.

A conserved role for the Drosophila Pax6 homolog Eyeless in differentiation and function of insulinproducing neurons

Jason Clements ${ }^{1}$, Korneel Hens ${ }^{1}$, Carmen Francis ${ }^{1}$, Ann Schellens ${ }^{1}$, Patrick Callaerts ${ }^{1}$

${ }^{1}$ Dept. of Human Genetics, K.U.Leuven \& VIB

Insulin/Insulin-like growth factor (IGF) signaling constitutes an evolutionarily conserved pathway that controls 
growth, energy homeostasis and longevity. In Drosophila melanogaster, key components of this pathway are the insulin-like peptides (Dilps). The major source of Dilps is a cluster of large neurons in the brain, the insulinproducing cells (IPCs). The genetic control of IPC development and function is poorly understood. Here, we demonstrate that the Pax6 homolog Eyeless is required in the IPCs to control their differentiation and function. Loss of eyeless results in phenotypes associated with loss of insulin signaling, including decreased animal size and increased carbohydrate levels in larval hemolymph. We show that mutations in eyeless lead to defective differentiation and morphologically abnormal IPCs. We also demonstrate that Eyeless controls IPC function by the direct transcriptional control of one of the major Dilps, dilp5. We propose that Eyeless has an evolutionarily conserved role in IPCs with remarkable similarities to the role of vertebrate Pax6 in b cells of the pancreas.

\section{III.20}

\section{Organization and Function of the Blood-Brain Barrier in Drosophila}

Daniel Engelen ${ }^{1}$, Christian Klämbt ${ }^{1}$

1 Institute of Neurobiology, University of Muenster, Badestr. 9, 48149 Münster, Germany

The correct function of a complex nervous system depends on an elaborate tuning between neuronal and glial cell types. One important necessity for an efficient electric transduction is the proper insulation of the nervous system. In Drosophila, this insulation is established by subperineurial glial cells, that are connected by septate junctions. Two additional cell types complete the functional blood-brain barrier (BBB), the perineurial glial layer, a layer of extracellular matrix, called neural lamella and the so called wrapping glia, that enwraps every single axon.

Here we demonstrate the influence of EGFR signaling for the proper formation of the blood-brain barrier in Drosophila. EGFR mutant animals show a leakage of the BBB. Similarly, reduction of EGFR function specifically in glia results in a loss of BBB integrity. This shows the cell autonomous need of EGFR signaling in glial cells and suggests a connection between EGFR signaling and septate junction formation. These findings are supported by glial specific activation of EGFR, leading to an increase in septate junction length assayed by TEM analysis.
Further results on the function of EGFR signaling for the maintenance of septate junctions will be presented.

The regulation of consecutive steps in Drosophila mushroom body axonogenesis by Neuroglian/Central brain deranged involves selective interactions with the ZO-1 homolog Polychaetoid

Tim Goossens ${ }^{1}$, Yuan Yuan Kang ${ }^{2}$, Gunther Wuytens ${ }^{3}$, Pascale Zimmermann ${ }^{3}$, Giulia Pollarolo ${ }^{3}$, Michael Hortsch $^{4}$, Patrick Callaerts ${ }^{1}$

1 Lab of Developmental Genetics, VIB/K.U.Leuven, Leuven, Belgium

2 Department of Cell Biology, Baylor College of Medicine, Houston, USA

3 Department of Human Genetics, VIB/K.U.Leuven, Leuven, Belgium

${ }^{4}$ Department of Cell and Developmental Biology, U. Michigan Medical School, Ann Arbor, USA

Drosophila mushroom bodies are a prominent neuropil in the central brain with important roles in complex behaviors such as learning and memory and visual context generalization. It is not well understood how during development extracellular cues and adhesive interactions are integrated to control neuron subtypespecific neuritogenesis. We here identify a role for neuroglian (nrg), the Drosophila L1CAM homolog, in mushroom body axonogenesis. We identify two central brain deranged (ceb) alleles as nrg-mutations and show that $\mathrm{ceb}$ and $\mathrm{nrg}$ alleles have similar mushroom body axon outgrowth, branching and guidance defects. Although $\mathrm{Nrg}$ is broadly expressed in the post-embryonic brain, its phenocritical period spans late larval and early pupal stages. By means of transgenic rescue experiments, we demonstrate that the non-neuron specific form of $\mathrm{Nrg}, \mathrm{Nrg}^{167}$, can rescue mushroom body peduncle formation, but that full rescue of the nrg-mutant phenotype requires the C-terminal most part of the neuronal specific $\mathrm{Nrg}^{180}$ isoform. MARCM and primary cell culture experiments are consistent with a partially cell-autonomous Nrg-function in MB development, in which the cytoplasmic region of $\mathrm{Nrg}^{180}$ is necessary for outside-in signaling. Genetic interaction tests and Far Western analysis show that this signaling pathway involves a direct interaction of $\mathrm{Nrg}^{180}$ with the ZO-1 homolog Polychaetoid (Pyd), revealing a novel role of a MAGUK protein in signal integration during axonal differentiation. 
III. 23

IRM-proteins in neural development II: Differential transport in neurons

Moritz He $\beta^{1}$, Kerstin Götz ${ }^{1}$, Rajneesh Srivastava ${ }^{1}$, Shamprasad Varija Raghu ${ }^{1}$, Kokil Chaudhary ${ }^{1}$, Karl-Friedrich Fischbach $^{1}$

${ }^{1}$ Faculty of Biology, Institute of Biology III, AlbertLudwigs-University Freiburg

The genes of the four IRM proteins IrreC/Rst, Kirre, SNS and $\mathrm{Hbs}$ are differentially expressed in neurons and glial cells. The apparent similarity at the level of the confocal microscope of the immunoreactivity pattern of all four IRM-proteins in the pupal optic neuropils is the result of highly specific transport of the proteins to the neuropil layers where synaptic contacts between fine dendritic and axonal arborizations are being made. Targeted expression of IrreC/Rst and Kirre in retinula cells leads to very strong immunoreactivity in their terminals, while this is not the case with SNS and Hbs. MARCM studies using irreC/rst and kirre Gal4 lines as well as cell type specific Gal4 lines reveal that these proteins are not localized in cell body fibres or axons, but accumulate in presumptive presynaptic arborization, e. g. in the case of $\mathrm{Tm} 2$ cells, overexpressed Kirre is transported mainly to the arborizations in M9 and to the axonal terminals in the lobula, while SNS and Hibris are not. In contrast overexpressed Hibris accumulates in the distal $\mathrm{Tm} 2$ arborization of the medulla. We misexpressed IrreC/Rst, Kirre and SNS also in neurons of the central brain. Here again IrreC/Rst and Kirre are transported into axonal terminals, while SNS is found in dendrites exclusively.

Postembryonic development of glial cells in dorsomedial brain neuroblast lineages.

Natalya Izergina ${ }^{l}$, Bruno Bello, Heinrich Reichert

${ }^{1}$ Biozentrum University of Basel

Specific dorsomedial (DM) lineages of the Drosophila brain that consist of an unusually large number of cells are produced by a novel type of neuroblast that amplifies proliferation through generation of intermediate progenitors (Bello et al., 2008; Bowman et al., 2008). Here we show that these neuroblasts generate not only neurons, but also a novel postembryonic brain glial cells. To analyse these glial cells, we use the MARCM-based clonal techniques for cell identification, determination of cell number and morphology as well as elucidation of specific gene action in the postembryonic brain. With these techniques, we also determine the time course of generation of these glia within the DM lineages and find that they are all generated in a specific temporal window as compared to the neuronal progeny of the lineages. Moreover, with MARCM techniques we document the expression of specific developmental control genes in these cells and then use clonal mutant techniques to eliminate or change the cell fate of these glial cells in the developing brain. Our findings demonstrate that the DM neuroblasts, which are novel in their mode of proliferation amplification, are also exceptional in that they represent the only currently known neuroglioblasts in the postembryonic brain.

Supported by SNSF.

III.27

Temporal regulation of Corazonin expression in lateral protocerebrum of Drosophila melanogaster

María Losada ${ }^{l}$, Marta Andrés, Alicia Gonzalo, Ignacio Monedero, Inmaculada Canal, Isabel Molina

${ }^{1}$ Departamento de Biología, Universidad Autónoma de Madrid

Corazonin is a neuropeptide found in almost all insect orders. Corazonin acts as a neurohormone when expressed by neurosecretory cells from the lateral protocerebrum, and it also functions as a neuromodulator when expressed by interneurons from the ventral ganglion. Corazonin pattern is well established in the species in which it has been studied, but little is known about the regulation of its expression. A previous work has shown that the transcription factor Squeeze is required in lateral protocerebrum cells to express Corazonin but not in the interneurons of the ventral ganglion. In the present work we show that restoring expression of Squeeze in LP cells is sufficient to rescue normal Corazonin expression in $s q z$ mutants. This squeeze requirement in LP cells is not related to cell survival. When apoptosis is inhibited in $s q z$ mutants by directing the expression of $\mathrm{p} 35$ to LP cells, Corazonin is not rescued. In addition we also show that in adult stages, but not during larval development, Corazonin expression in the lateral protocerebrum depends on retrograde transport via BMP signaling as it has been previously reported in the regulation of other neuropeptides.

III. 28

Bundling actin to shape up dendrites

Julia Negele $^{1}$, Gaia Tavosanis ${ }^{1}$ 
${ }^{1}$ Molecular Neurobiology, MPI of Neurobiology, Martinsried, Germany

The sophisticated branching pattern of dendrites is achieved by the interplay of a number of different molecules including guidance cues, transcription factors and cytoskeletal molecules. However, the cytoskeletal regulation of dendritic branching is poorly understood. Here we show that the actin-bundling molecule singed, the Drosophila fascin homologue, affects the branching pattern of two different types of PNS neurons. In class I neurons, which show a simple dendritic tree, the absence of the protein leads to an increased number of higher order branches without affecting the number of primary and secondary branches. In contrast, the more complex dendritic trees of class III neurons show less spike-like protrusions if the singed protein level is reduced. In the singed null mutant larva the density of the spike-like protrusions is even more reduced but additionally the complexity of the main branches is increased. Thus, actin bundling appears to be necessary for the formation of class III spikes, but stable actin bundles suppress class I high order branches and the ectopic outgrowth of main branches of class III neurons. Interestingly, the most complex PNS neurons, class IV neurons, are not affected by singed. Our investigations suggest that the cytoskeletal mechanisms underlying the formation of different types of branches in diverse neuronal classes are different.

\section{A Loss of Function Screen for Genes Involved in Dendrite Morphogenesis}

Annemarie North ${ }^{1}$, Matthias Landgraf ${ }^{1}$

${ }^{1}$ Department of Zoology, University of Cambridge

In order to produce a functional nervous system, it is essential that neurons are able to find and make connections with their appropriate pre- and postsynaptic partners. We are interested in understanding more about how the postsynaptic dendrites of neurons achieve their characteristic arborisations.

The approach taken is a loss of function screen of D. melanogaster motor neuron dendrites. Using a modified MARCM technique in combination with recombinant FRT Deficiency chromosomes that we have generated, we are screening the second chromosome for genes involved in dendrite morphogenesis. The modified MARCM technique allows us to make a subset of motor neurons homozygous for a deficiency and visualise them in a wild type background - preferentially targeting genes that are intrinsically required for proper dendrite morpho- genesis. Trials with robo and short stop mutants showed that mutant phenotypes can be identified based on their dendritic arbors using this technique.

Deficiency chromosomes from the Bloomington stock centre are recombined with FRT sites to generate lines that can be used in the MARCM screen. Currently, 102 Deficiency lines recombined with FRT chromosomes are available, providing coverage of approximately $57 \%$ of the second chromosome.

Thus far we have identified 5 genomic regions that have a phenotype when absent. Here we present work in progress to identify the gene(s) responsible for a targeting phenotype observed with the removal of one of these regions.

III.30

\section{The Transmembrane Protein Golden Goal Cooperates with Hu Li Tai Shao to Guide Photoreceptor Axons}

Stephan Ohler ${ }^{1}$, Satoko Suzuki ${ }^{1}$, Tatiana Tomasi ${ }^{1}$, Takashi Suzuki ${ }^{1}$

1 Axonal Guidance and Neuronal Connectivity, Max Planck Institute of Neurobiology

Mammalian Adducin is a ubiquitously expressed cytoskeletal protein that links short Actin filaments to Spectrin tetramers. Hu Li Tai Shao (Hts), the Drosophila homolog of Adducin, was found to physically interact with the cytoplasmic part of Golden Goal (Gogo), a transmembrane protein that plays an important role in photoreceptor axon guidance of the developing visual system: Gogo is required for the proper spacing of photoreceptor axons and for correct target layer recognition. Adult $h t s$ mutant animals show a phenotype similar to, but less severe than gogo mutants, with photoreceptor axons crossing each other and forming clumps and gaps in the medulla. Binding to Gogo is mediated by the head or neck domain of Hts, which are common to all Hts isoforms and therefore, all of the Hts isoforms are able to bind to Gogo in vitro. However, isoform-specific rescue experiments revealed that the phenotype found in hts mutant animals can be partially rescued only by the Hts isoforms Hts. PB or Hts. PD, which most closely resemble mammalian Adducin in the tail domain. Neither the more distantly related isoform Hts. PA, which has a very long and specialized tail domain involved in oogenesis, nor Hts. PC with its truncated tail domain are able to rescue the $h t s$ phenotype.

These results suggest that Hts could be required for the correct localization of Gogo or that Gogo exerts its function in photoreceptor axon guidance by regulating the Actin-Spectrin cytoskeleton via Hts. 
III.31

\section{A Catalogue of Interneuronal Cell Types in the Late Embryonic Nervous System of Drosophila}

Christof Rickert $^{1}$, Tomas Kunz ${ }^{1}$, Kerri -Lee Harris ${ }^{2}$, Paul Whitington $^{2}$, Gerd Technau ${ }^{3}$

${ }^{1}$ Institute of Genetics, University of Mainz

2 Dept. of Anatomy and Cell Biology, University of Melbourne

${ }^{3}$ Institute of Genetics, University of Mainz

In the Drosophila embryonic ventral nervous system each hemineuromere derives from a set of 30 neuroblasts and comprises about 350 neurons and 30 glial cells. While the entire lineage of each neuroblast is known and all glial cells and all motoneurons have been identified individually, the vast amount of the more than 300 interneurons are not described on a single cell level. Here we present a detailed description of the intire population of interneurons. It is based on labelling by iontophoretic dye injections of about 1000 cells in early stage 17 flat preparations. This set of data allows on the one hand to assess the number and morphology of the different interneuronal cell types and to estimate a single interneurons' variability regarding cell body position or exact axonal/dendritic morphology. On the other hand it is now possible for the first time to systematically test for correlations, e.g. between cell body positions and projection patterns or positions of axons within the commissures and the connectives. This helps clarifying the structural organization of neuromeres en the ventral nerve chord. Additionally our data provide the basis for future mutant analysis and will facilitate the identification of neuronal circuits. Supported by the DFG (Te 130/7-5)

\section{III.33}

\section{Genetic analysis of glial cell development in Droso- phila}

Imke Schmidt ${ }^{1}$, Marion Silies ${ }^{1}$, Christian Klämbt ${ }^{1}$

${ }^{1}$ Institute of Neurobiology, University of Muenster

In Drosophila larvae, 65 individually recognizable glial cells are found in each hemineuromer, which exert a growing number of functions during the development and subsequent functioning of the nervous system. Due to this low cellular complexity, Drosophila represents a particular well-suited model to study glial cell biology. We have characterized all morphological glial cell classes in detail using flipout, MARCM and EM approaches. To dissect glial biology we initiated a number of approaches. The establishment of the FRT/ FLP system allows the generation of mosaic animals with a homozygous mutant tissue in an otherwise phenotypically wild type background. Following the generation of a glial cell specific FLP source we generated flies with homozygous mutant glial cells and screened for those mutations that interfered with normal viability. The method was tested using mutations genes known to be essential for glia such as pointed as test case. However, an enormous developmental plasticity compensated all physiological defects caused by even large mutant glial cell clones. To nevertheless determine the genes required during glial cell development we employed a collection of UAS-dsRNA strains available in the stock centers (VDRC and NIG-fly). We started screening genes encoding membrane-associated proteins and found that about $15 \%$ of the lines compromise viability and are currently assayed in further detail. First results of this screen will be presented.

III.34

Identity, origin, and migration of peripheral glial cells in the Drosophila embryo

Christian von Hilchen ${ }^{1}$, Ruth Beckervordersandforth ${ }^{1}$, Christof Rickert ${ }^{1}$, Benjamin Altenhein ${ }^{1}$, Gerhard Technau $^{1}$

${ }^{1}$ Institute of Genetics, University of Mainz

Peripheral glial cells (PGs) are required for the correct establishment of the peripheral nerves and the formation of the blood/nerve barrier. Most of the embryonic PGs originate in the CNS, and they migrate over long distances to align along and finally ensheath the peripheral nerves. In order to gain more insights into the development of the PGs on the cellular level, we studied the spatial and temporal pattern, identity, migration, and origin of all PGs in truncal segments of wildtype embryos. We present a catalogue of markers, whose expression patterns indicate the establishment of individual cell identities and allow their discrimination. We uncovered the origin of each of the PGs by cell lineage analysis and linked them to identified CNS neuroblasts and sensory organ precursor cells. Using confocal 4D microscopy and GFP expression, we traced in vivo the migratory behaviour of the individual PGs during the course of embryonic PNS development. The order in which the individual PGs migrate into the periphery and their final positioning shows only minor variations. This detailed description of peripheral glia development and migration provides the basis for the examination of phenotypes on a single cell level under various mutant and experimental conditions. 
IV.P

\section{Neurobiology of the Drosophila circadian clock}

Marc Ruben ${ }^{1}$, Ben Collins ${ }^{1}$, Mark D. Drapeau ${ }^{1}$, Dogukan Mizrak $^{1}$, Justin Blau ${ }^{1}$

${ }^{1}$ Biology Department, New York University

Studies of circadian rhythms in Drosophila are an excellent example of how forward genetics can identify key genes that regulate behavior. Genetic and biochemical studies have given us a good understanding of the transcriptional-translational feedback loops that sustain rhythmic clock gene RNA and protein accumulation in pacemaker neurons even in constant darkness. However, it is unclear how the information encoded by these molecular clocks is transduced into time-of-day information for the animal. A long-standing idea is that the molecular clock also regulates the rhythmic expression of clock output genes that lead to daily changes in pacemaker neuron activity. To identify potential clock outputs, we performed GeneChip analysis on purified Drosophila pacemaker neurons. One of the most interesting rhythmically expressed genes that we identified in clock neurons is $I r$, which encodes an inward rectifier $\mathrm{K}+$ channel that contributes to the resting membrane potential of neurons. Gain-of-function and loss-of-function behavioral experiments indicate that rhythmic $I r$ expression is a major contributor to pacemaker neuron activity. We have also been using the relatively simple circadian system in Drosophila larvae as a model to understand how different clock neurons communicate with each other to generate robust rhythms.

IV.4

The stimulatory heterotrimeric G-protein $\mathbf{G} \alpha_{\mathrm{s}}$ is involved in olfactory signal transduction in Drosophila

Ying Deng ${ }^{1}$, Weiyi Zhang ${ }^{1}$, Gisselmann Guenter ${ }^{1}$, Hanns Hatt $^{1}$, Eva M. Neuhaus ${ }^{1}$

${ }^{1}$ Cell Physiology, Ruhr University Bochum

Olfactory signal transduction starts with the activation of olfactory receptors, which are known to recognize of a wide range of structurally highly variable substances. While vertebrate olfactory receptors are 7 transmembrane proteins which activate heterotrimeric G-proteins after ligand binding, olfactory receptors in Drosophila were reported to have an inverse membrane topology compared to classical G-protein coupled receptors, with an intracellular N- and extracellular C-terminus. Moreover, it was show recently that the receptors can function as ligand gated ion channels. Controversial findings were reported concerning the additional involvement of heterotrimeric G-proteins in olfactory receptor signaling. One arising question is therefore, whether these 7 transmembrane receptors also couple to heterotrimeric G-proteins, in addition to the reported novel way of signaling. Our data involving in vivo electrophysiological recordings and protein redistribution, as well as investigations using recombinantly expressed olfactory receptors, now demonstrate that olfactory receptor signaling in Drosophila indeed involves G-proteins for signal transduction. Moreover, our results provide compelling evidence that the stimulatory $\mathrm{G} \alpha_{\mathrm{s}}$ protein is involved in odorant detection. In conformity with $G \alpha_{s}$ signaling we could show that increased cAMP levels lead to excitation of the olfactory sensory neurons.

\section{IV.5}

The Diversity of the Paralytic Voltage-Gated Sodium Channel in Drosophila

Wei-Hsiang Lin $^{1}$, Duncan E. Wright ${ }^{1}$, Richard A. Baines ${ }^{1}$

${ }^{1}$ Faculty of Life Sciences, University of Manchester, Manchester, M13 9PT, UK

The voltage-gated sodium channel in Drosophila is encoded by a single gene, termed paralytic (para). Currently 11 alternatively-spliced exons have been reported that serve to increase functional channel diversity. However, which exon compositions are present at specific developmental stages and how each transcript contributes to alter channel properties are not well understood. In order to determine the most common splice isoforms present in late stage 17 embryos, RT-PCR was used to isolate 50 clones of the complete open reading frame. Comparing the exon compositions of these sequences revealed 27 unique splice isoforms. The channel properties of the most common variants were determined by two-electrode voltage clamping following expression in Xenopus oocytes. Isoforms containing spliced exons $\mathrm{j}$ and e activate at more depolarized membrane potentials, while those containing exon $\mathrm{f}$ activate at hyperpolarized potentials. By contrast, splice variants containing exon $h$ inactivate at more depolarized membrane potentials. Finally, we show that inclusion of exons $\mathrm{k}, \mathrm{l}, \mathrm{h}$ or $\mathrm{c}$ markedly affects the size of the non-inactivating persistent sodium current. These data imply that the capacity for fine-tuning of the properties of voltage-gated sodium currents, in response to changing demands, might be regulated by expressing differing para sodium channel splice variants in the Drosophila CNS. 
IV.6

Expression analysis of the three G-protein subunits: Galpha, Gbeta and Ggamma, in the olfactory receptor organs of adult Drosophila.

Tamara Boto ${ }^{1}$, Carolina Gomez-Diaz ${ }^{1}$, Esther Alcorta ${ }^{1}$

${ }^{1}$ Dep. Functional Biology, University of Oviedo

In Drosophila, studies in heterologous systems discuss whether olfactory receptors interact with G-proteins to trigger transduction or if they act as nucleotide-gated channels themselves. As a preliminary work to unravel "in vivo" this controversy, here we supply a thorough description of the expression of all known Drosophila melanogaster G-proteins in olfactory receptor organs: third antennal segment and maxillary palps. Heterotrimeric $\mathrm{G}$ proteins are composed by three subunits, which mediate many cellular signaling processes. We analyzed the variants of the alpha, beta, and gamma subunits corresponding to the different genes and their transcripts, by RT-PCR. We found that all tested Galpha protein genes showed expression in the antennae but $\mathrm{G}_{\mathrm{f}}$ was not detected in maxillary palps, and that all Gbeta and Ggamma are expressed in antennae and palps except $\mathrm{G}_{\text {beta76C }}$. Differential expression of Galpha variants, joined to variation in regulatory sequences among the mRNAs suggest that especific Galpha proteins may play a role in the generation of different types of olfactory neuron signaling. To gain some insight on this subject, inmunohystochemical studies of specific expression for different Galpha and Gbeta proteins in antennal sections have been done, showing generalized expression of these proteins in neurons and glial cells. Supported by the Spanish Ministry of Education and Science.

\section{IV.7}

The cyclic nucleotide-gated channel-like, CNGL, in the olfactory reception of Drosophila melanogaster

Carolina Gomez-Diaz ${ }^{1}$, Fernando Martin ${ }^{1}$, Esther Alcorta $^{1}$

${ }^{1}$ Functional Biology (Genetics), Universidad de Oviedo

Cyclic nucleotide-gated channels allow ion flux trough the plasmatic membrane in photoreceptors and olfactory receptor neurons (ORNs) in vertebrates. Odorant stimulation opens $\mathrm{CNG}$ channels through an intracellular AMPc increase depolarizing the membrane. In insects, there is evidence of cyclic nucleotides production due to olfactory stimulation. In Drosophila, this type of channels, as CNG, with GMPc preference, and CNG channel-like, with AMPc preference, has been found. The role of AMPc in the olfactory reception of insects has been confirmed by molecular, physiological and behavioral experiments. In this work, we try to determine if CNGL is involved in the olfactory reception of Drosophila "in vivo". We have demonstrated the expression of the $\mathrm{cngl}$ gene in the main and secondary olfactory organs (antennae and maxillary palps) by RT-PCR. The CNGL protein has been found by immunohistochemistry in every neuron of the third antennal segment. The staining appears in the entire antenna but it is more abundant at the base of the basiconic and thricoid sensilla. Functionally, electroantennogram (EAG) analysis in hybrids that overexpress cngl in most of the ORNs showed significant differences in amplitude and kinetics compared to the controls in response to ethyl acetate, acetone and ethanol pulses. All these data suggest that the CNG channel-like (CNGL) may play a role in the first steps of olfactory transduction. Funded by The Spanish Ministry of Education and Science and the PCTI program of the Principado de Asturias.

IV.8

Function of the Shaw potassium channel within the Drosophila circadian clock

James J. Hodge ${ }^{l}$, Ralf Stanewsky ${ }^{2}$

${ }^{1}$ Physiology and Pharmacology Department, University of Bristol

${ }^{2}$ School of Biological and Chemical Science, Queen Mary University of London, London, UK

Electrical activity is important for the generation of rhythmic behavior by Drosophila clock neurons. To determine the mechanisms regulating resting membrane potential (RMP) in the native clock we modulated the function of Shaw, a widely expressed neuronal $\mathrm{K}+$ channel known to regulate RMP in Drosophila central neurons that we show to be endogenously expressed in clock neurons. Differential use of clock gene promoters allowed the expression of transgenes that either increase or decrease Shaw function in different clusters of clock neurons. Under LD conditions, increasing Shaw levels in all clock neurons or in subsets of clock neurons ( $\mathrm{LNd}$ and DNs or DNs alone) increases locomotor activity at night. In free-running conditions this results in arrhythmic locomotor activity without disruption of the molecular clock. Reducing Shaw in the DN alone caused a dramatic lengthening of the behavioral period. Changing Shaw levels in all clock neurons also disrupts the rhythmic accumulation and levels of Pigment Dispersing Factor (PDF) in the dorsal projections of LNv neurons. However, changing Shaw levels solely in LNv neurons had little effect on locomotor activity or rhythmic PDF accumula- 
tion. Based on our results it is likely that Shaw modulates pacemaker and output neuronal electrical activity that controls circadian locomotor behavior by affecting rhythmic release of PDF. The results support an important role of the DN clock neurons in Shaw-mediated control of circadian behavior.

IV.9

Distinct incorporation behaviours of glutamate receptors at individual synapses of Drosophila NMJs.

Omid Khorramshahi ${ }^{1}$, Schmid Andreas ${ }^{2}$, Schwarz Tobias $^{1}$

${ }^{1}$ Bio-Imaging Center, Rudolf-Virchow-Zentrum

${ }^{2}$ ENI Göttingen

Changes in numbers of structural and functional distinct non-NMDA glutamate receptors (GluRs) control maturation and plasticity of single synapses during memory formation. At the neuromuscular junction (NMJ) of Drosophila two distinct ionotropic glutamate receptor complexes transduce the presynaptic excitatory input to the muscle membrane. These receptors contain different subunits (GluRIIA \& GluRIIB) and offer distinct physiological properties. By applying in vivo imaging techniques in Drosophila larvae it has been shown that the incorporation dynamics of GluRIIA and GluRIIB at the NMJ are distinct and dependent on evoked presynaptic glutamate release. We are studying the dynamics of functionally mutated versions of the two receptor complexes which will give further insights in postsynaptic plasticity mechanisms.

\section{V.P}

New insights into axon extension and filopodia formation in a Drosophila growth cone model

Andreas Prokop ${ }^{1}$, Natalia Sanchez-Soriano ${ }^{1}$, Catarina Pimentel $^{1}$, Mark Travis ${ }^{1}$, Federico Dajas-Bailador ${ }^{1}$, Alan Whitmarsh ${ }^{1}$, Jozsef Mihaly ${ }^{2}$, Ulrike Haessler ${ }^{2}$

${ }^{1}$ Faculty of Life Sciences, The University of Manchester

${ }^{2}$ Hungarian Academy of Sciences

To build neuronal networks, nerve cells extend long neurites in a highly regulated fashion, executed by growth cones (GC) at their tips. Here we report the establishment of a Drosophila GC model based on embryonic primary neurons which provide us with robust quantifiable features and properties similar to GCs of other animal models. Genetics can be applied efficiently in this context, and we focussed such studies on two major aspects: mechanisms of filopodia formation and the microtubule-related machinery of axon extension. 1) We demonstrate a requirement for the Formin Daam in filopodia formation. Furthermore, we demonstrate that the G-actin binding protein Profilin is essential for filopodia elongation, and genetic interaction studies reveal a further function in filopodia formation. 2) Also the actin-microtubule linker Short stop (Shot) is required for filopodia formation, a function clearly distinct from a second role of Shot in organising microtubules in axons and growth cones. Only the latter function correlates with axon length and suggests Shot to be an essential constituent of the machinery mediating axon extension. Our studies of the Shot orthologue ACF7 in Neuro2A cells and cortical neurons clearly demonstrate that this role is conserved in mammals. In conclusion, this GC model adds essential subcellular resolution to existing in situ models of neuronal growth in Drosophila. Supported by: BBSRC, Wellcome Trust, Royal Commission 1851, FCT, EMBO, HHMI

V.2

Cell-autonomous determination of photoreceptor synaptic organelles in Drosophila R1-R6 photoreceptor terminals mis-targeted to the medulla.

Tara N. Edwards ${ }^{1}$, Ian A. Meinertzhagen ${ }^{2}$

${ }^{1}$ Dept. of Biology, Dalhousie University

2 Dept. of Neuroscience, Dalhousie University

Fly photoreceptors are of three types. Large, outer R1-R6 photoreceptors terminate in the lamina and form a ring of rhabdomeres that express Rh1. Within these, the inner R7 and $\mathrm{R} 8$ have smaller rhabdomeres that express Rh3/Rh4 or $\mathrm{Rh} 4 / \mathrm{Rh} 5$, respectively, and axons that innervate the medulla. A previous report showed that exogenous expression of Runt in subsets of photoreceptors results in incorrect targeting of R1-R6 terminals to the medulla (Kaminker et al., 2002). Using the GAL4/UAS system, we find that Runt over-expression can induce a change in photoreceptor cell fate, from outer to inner. While some Rh1-expressing R1-R6 photoreceptor axons terminate in the medulla, normal target cells within the lamina nevertheless persist and express cell-specific markers. R1-R6 terminals form reciprocal synaptic contacts with their normal lamina targets, but supernumerary terminals targeted to the medulla also form synapses. Thus, tetrads form both within the lamina and at supernumerary terminals in the medulla. Furthermore glia invaginate terminals at both sites, to form capitate projections, sites of vesicle endocytosis. Unlike wild-type, the size and shape of the capitate projection head is identical in both lamina and ectopic medulla terminals, even though those 
in the medulla receive invaginations from foreign glia. The uniformity of synapses and glial invaginations indicates that the presynaptic photoreceptor terminal determines these synaptic organelles cell-autonomously.

\section{V.3}

Imb controls synapse sepcificity in the Drosophila ellipsoid body

Chien-Kuo Chen $^{1}$, Cheng-Ting Chein ${ }^{1}$

1 Institute of Molecular Biology, Academia Sinica, Taipei, Taiwan

The ability to select appropriate synaptic partners is essential for complex neural networks. The mechanisms that control these processes are not well known. We found that the Drosophila ellipsoid body (EB) is a suitable system to study synaptic specificity. First, axon terminals of R neurons are highly structured and easy for observations of presynaptic phenotypes in the EB. Second, the different $\mathrm{R}$ neuron types $(\mathrm{R} 1-4)$ are defined by their presynaptic patterns. Lastly, presynaptic patterns of $\mathrm{R}$ neurons are characterized by the three factors $(\kappa, \mathrm{r}$ and $\mathrm{h})$ in a cylindrical coordinate system. Here, we show that transcription factor Immobile (Imb) is required for synaptic specificity of $\mathrm{R}$ neurons. Interestingly, Imb levels are sufficient to control the presynaptic patterning in the anterior-posterior axis of EB but not in the frontal plane of the EB. We propose that $\mathrm{R}$ neurons use two different pathways to mediate the synapse specificity in the space.

\section{V.4}

Erect wing restricts synaptic growth presynaptically and interacts with multiple signaling pathways

Matthias Soller ${ }^{1}$, Kalpana White ${ }^{2}$, Irmgard Haussmann ${ }^{1}$

${ }^{1}$ School of Biosciences, University of Birmingham

2 Biology Department, Brandeis University

Formation of synaptic connections is a dynamic and highly regulated process. Synaptic growth regulation requires a balance of stimulatory and restrictive signals. How cellular signals are integrated into gene expression programs remains one of the central questions in biology. We have identified the Erect wing transcription factor to restrict synaptic growth at third instar NMJ's using a clonal analysis for loss of function and temporal over-expression for gain of function. This analysis shows a pre-synaptic and cell-autonomous role for ewg in restricting synaptic growth. To gain further insights into the role of ewg in regulating synaptic growth and to determine general principles operating in this biological process, we used a functional genomics approach. Analysis of mutants in ewg differentially regulated genes for defects in synaptic growth revealed predominantly genes involved in transcriptional and post-transcriptional regulation of gene expression in addition to genes assigned with neuronal functions. Among ewg differentially regulated genes with synaptic growth defects are a number of genes involved in signaling pathways. We extended the clonal analysis of ewg loss of function to test for genetic interactions with these signaling pathways. Our analysis shows that none of these signaling pathways acts independently of ewg suggesting that an extensive regulatory network controls the expression of a limited number of effector genes to regulate synaptic growth.

V.5

The F-BAR protein syndapin promotes morphogenesis of an endogenous postsynaptic membrane system in drosophila

Vimlesh Kumar ${ }^{1}$, Suneel Reddy Alla ${ }^{2}$, Debjani Bhar ${ }^{2}$, KS Krishnan $^{2}$, Mani Ramaswami ${ }^{3}$

${ }^{1}$ Institute of Neuroscience, University of Dublin Trinity College, Ireland

2 Tata Institute of Fundamental Research, Mumbai, India

${ }^{3}$ Institute of Neuroscience, University of Dublin Trinity College, Ireland and Department of Molecular and Cellular Biology, University of Arizona, Tucson, USA

The EFC/F-BAR protein family have postulated functions in endocytosis, membrane tubulation and actin regulation. Among these, syndapins have predicted function in synaptic vesicle recycling. To understand in vivo functions of F-BAR proteins in general, and of syndapin in particular we have analysed NMJ synapses of Drosophila variants either lacking or expressing mutant forms of syndapin.

Our functional analyses (electrophysiology and live synaptopHluorin imaging) show that, contrary to prevailing expectation, synd is dispensable for synaptic-vesicle recycling and indeed undetectable at the Drosophila motor terminal. However, Drosophila synd appears function downstream of, or parallel to, dPAK and Dlg in the morphogenesis of postsynaptic-membrane system.

Like mammalian syndapin, Drosophila Synd binds dynamin and WASp. Our data indicate that the SH3 domain of Synd is required for postsynaptic targeting of the protein, but not for membrane tubulation in vivo. The F-BAR domain is sufficient for membrane reorganization in muscles, through a process that required basic residues 
postulated by recent structural studies to reside on the concave surface of the protein and to participate in binding to purified liposomes.

Our findings suggest that: a) syndapin is not a general component of synaptic vesicle endocytosis; b) syndapin uses membrane-remodeling mechanisms suggested by previous in vitro and structural analyses to promote formation of a tubulolamellar postsynaptic membrane system.

\section{V.6}

Drosophila Ankyrin 2 organizes a presynaptic cytoskeletal scaffold that is essential for synaptic stability

Hermann Aberle ${ }^{1}$, Bernd Goellner ${ }^{1}$, Iris $\mathrm{Koch}^{2}$, Heinz Schwarz ${ }^{2}$

${ }^{1}$ Institute for Neurobiology, University of Muenster

2 MPI Developmental Biology, Tuebingen

Once synapses have formed, functionally important connections have to be maintained and stabilized, mostly by transsynaptic adhesion complexes that are anchored in the underlying cytoskeleton. The Drosophila neuromuscular junction (NMJ) serves as a model system for central synapses in the vertebrate brain, as both synapse types share molecular and structural features. In a mutagenesis screen for regulators of synaptic stability, we recovered mutations in Drosophila ankyrin 2 (ank2) affecting two giant Ank2 isoforms that are specifically expressed in the nervous system, and that are tightly associated with the presynaptic membrane cytoskeleton. ank2 mutant larvae show severe deficits in the stability of NMJs, resulting in a reduction in overall terminal size. Synaptic boutons are withdrawn and presynaptic active zones are disassembled. In addition, lack of Ank2 leads to disintegration of the synaptic microtubule cytoskeleton. Microtubules and microtubule-associated proteins fail to extend into distant boutons. Interestingly, Ank2 functions downstream of Spectrin in the anchorage of synaptic microtubules, providing a cytoskeletal scaffold that is essential for synaptic stability.

\section{V.7}

\section{A Presynaptic Gain Control Mechanism Fine-Tunes Olfactory Behavior}

Cory Root $^{1}$, Kaoru Masuyama ${ }^{1}$, David Green ${ }^{1}$, Lina Enell $^{2}$, Dick Nassel ${ }^{2}$, Chi-Hong Lee ${ }^{3}$, Jing Wang ${ }^{1}$

${ }^{1}$ Division of Biological Sciences, University of California-San Diego
2 Dept of Zoology, Stockholm University

${ }^{3}$ National Institute of Health

Early sensory processing can play a critical role in sensing environmental cues. We have investigated gain control in the first step of olfactory processing in Drosophila. Here we report that Drosophila olfactory receptor neurons (ORNs) express the $\mathrm{GABA}_{\mathrm{B}}$ receptor $\left(\mathrm{GABA}_{\mathrm{B}} \mathrm{R}\right)$. Using two-photon microscopy, we find that $\mathrm{GABA}_{\mathrm{B}}$ signalling negatively modulates presynaptic calcium influx and synaptic transmission. Furthermore, blocking $\mathrm{GABA}_{\mathrm{B}} \mathrm{R}$ and knocking down expression in ORNs reduces the dynamic range of ORN synaptic transmission that is matched by similar modulation of projection neuron response, suggesting a presynaptic mechanism to expand the dynamic range of a sensory system. Strikingly, we find that different ORN channels have different levels of presynaptic inhibition. In the extreme, the ORNs that sense the aversive odorant $\mathrm{CO}_{2}$ exhibit no presynaptic inhibition, and the pheromone sensing ORNs receive strong presynaptic inhibition. Finally, we find that $\mathrm{GABA}_{\mathrm{B}} \mathrm{R}$ mediated gain control enhances odor object localization in a mating assay. Our findings reveal that different sensory channels have different preset levels of presynaptic inhibition, which allows heterogeneous synaptic gain control to meet ecological needs.

V.8

Evolution of the synapse proteome

J Douglas Armstrong ${ }^{1}$, Bilal Malik ${ }^{1}$, Andrew Pocklington ${ }^{1}$, Richard Emes ${ }^{2}$, Seth Grant ${ }^{3}$

${ }^{1}$ School of Informatics, University of Edinburgh

2 Centre for Applied Entomology and Parasitology, Keele University, UK

${ }^{3}$ Wellcome Trust Sanger Institute, Cambridge, UK

Large-scale comparative proteomics has provided new insights into the structure and composition of the synapse proteome in both fly and mouse. Here we combined proteomic analysis (mass-spec) of the complex with expression analysis of the components in fly and mouse brain at RNA and protein levels. Both contain tightly bound signalling complexes containing of the order 200 proteins held together by PDZ-domain scaffolding proteins such as DLG and PSD95. It is estimated that the evolutionary lineages of arthropods and vertebrates separated 290 million years ago yet both complexes show remarkable similarity. Components of generic cellbiological processes regulated during synaptic plasticity (e.g. transcription and translation) were found to be 
predominantly of pre-metazoan origin. However the proto-synapse of early metazoans underwent molecular expansion in both arthropod and vertebrate lineages. These adaptations, which are more pronounced in vertebrates, primarily involved gene family expansion and diversification of signalling/structural components (receptors, scaffolders, cytoskeletal, adhesion and signal transduction proteins). Phyla specific innovations account for the most variable expressed proteins in both vertebrate and arthropod brains. Proteins found in both, yet absent in single cell eukaryotes, are expressed in brain regions that share basic common function across vertebrates and arthropods.

V.9

Glutamatergic signaling in the Drosophila mushroom body during the first week after eclosion

Irina Sinakevitch ${ }^{1}$, Serge Birman ${ }^{1}$

${ }^{1}$ Genetics of Neurotransmission, IBDML-Developmental Biology Institute, CNRS-Universite de la Mediterranee, Campus de Luminy, Case 907, F-13288 Marseille Cedex 9, France.

The mushroom body (MB) is a paired insect brain center located in the protocerebrum required for olfactory learning and memory. The MB structure is made up by intrinsic neurons (Kenyon cells) and consists of calyx, pedunculus and lobes (alpha, alpha', beta, beta', and gamma). In Drosophila, we previously observed that L-glutamate (Glu) is expressed by the last born Kenyon cells in the alpha and beta lobe cores for the first hours after eclosion, from where this aminoacid disappears after 24 hours. In order to understand the role of glutamate in MB development, we monitored Glu-like, dNR1 (NMDA receptor subunit), dVGluT (vesicular Glu transporter) and glutamine synthetase (GS) immunoreactivities during the first week of adult life. We found that dVGluT is present in extrinsic processes of the alpha and gamma lobes and spur region of the pedunculus but not in the Kenyon cells themselves. dNR1 immunostaining is located in the vicinity of dVGluT expression and its expression varied over the first week. Anti-GS stained glial cells are located close to the Glu-expressing last born Kenyon cells immediately after eclosion, then distribute equally all over the MB lobe neuropil and around the lobes. The MB calyx, in which input from the antennal lobe projection neurons are received, expresses very few dNR1- and dVGluT-positive processes. In contrast, these proteins are expressed at a high level in the lateral horn. This research was funded by FRM UFP20060306548 to IS.
V.10

How does Tan maturation and reaction kinetic affect histamine recycling from carcinine?

Silvia Aust ${ }^{l}$, Florian Brüsselbach ${ }^{1}$, Stefanie Pütz ${ }^{1}$, Bernhard T. Hovemann ${ }^{1}$

${ }^{1}$ AG Molekulare Zellbiochemie, Ruhr University Bochum

In the visual system of Drosophila, histamine is the neurotransmitter used for stimulus transmission from retina photoreceptor cells to postsynaptic neurons. There is ample experimental evidence supporting the idea of a histamine recycling pathway in a shuttle between photoreceptor axonal endings and surrounding epithelial glia. Two enzymes are involved in the recycling process, Ebony and Tan. Tan has been localized to photoreceptor cells, where it hydrolyses b-alanyl-histamine (carcinine) to b-alanine and histamine. Active Tan, as a member of the family of cysteine peptidases, is generated in a slow maturation process of internal proteolytic cleavage. We investigate the contribution of this maturation process and of the reaction kinetics of Tan on histamine recycling and visual performance. In aiming at these goals we are pursuing two strategies. First, we are generating maturation mutants in vitro. After transformation into the genome they will be used to investigate the effect of Tan processing on visual performance in vivo. In addition, Tan purification schemes are outlined for kinetic and structural investigations. Here, we report on the characterization of Tan maturation mutants and on an expression and purification scheme for Tan.

V.11

SRPK3-a new serine/threonine protein kinase in Drosophila melanogaster causes different defects

Alice Bloch ${ }^{l}$, Mandy Jauch ${ }^{l}$, Vanessa Nieratschker ${ }^{1}$, Erich Buchner ${ }^{l}$

${ }^{1}$ Genetics, University of Wuerzburg

Aggregates of the active zone protein Bruchpilot (BRP) are caused in larval motoneurons due to a mutation in an uncharacterized serine-/threonine kinase. Sequence analysis showed significant homologies between this kinase and mammalian SR protein kinases. The Drosophila gene was named serine-arginine protein kinase 3 gene (Srpk3). By RT-PCR four different mRNAs have been detected which are formed due to two independent transcription start sites and one alternatively spliced exon. SRPK3 is able to phosphorylate a synthetic SR-peptide in vitro and may therefore represent a Drosophila SR protein kinase. 
Elimination or reduction of SRPK3 lead to BRP aggregates in larval motoneurons but the fast axonal transport is not disrupted. Overexpressed SRPK3 co-localizes with BRP at active zone. A direct interaction of BRP and SRPK3 could not be shown. Behavioural assays provided additional evidence for a possible interaction between the two proteins because both the mutation of SRPK3 and its overexpression lead to similar behavioural deficits in walking, negative geotaxis, flight ability and reduced life span in adult flies as observed for BRP-RNAi knockdown flies. Flight inability, walking behaviour and negative geotaxis could be fully rescued by cDNA. The reduced life span phenotype could be rescued partially. Two of our current projects are the identification of interaction partners for SRPK3 via Cytotrap and an investigation of the ultrastructure of larval motoneurons using electron microscopy.

\section{V.12}

\section{Sphingolipid regulation of synapse structure and function}

Laura Briggs ${ }^{1}$, Matt Oswald ${ }^{1}$, Graeme Davis ${ }^{2}$, Sean T Sweeney ${ }^{1}$

${ }^{1}$ Department of Biology, University of York

${ }^{2}$ Department of Biochemistry, University of California

Sphingolipids are proposed to have a role in aspects of synapse structure, function and degeneration. However, any functional role has yet to be determined, despite an observed abundance of these lipid species at synapses. We have initiated a study of the structure and function of a sphingolipid deficient synapse in Drosophila, using mutations in a subunit of the heterodimeric serinepalmitoyltransferase enzyme, lace (sptII), which catalyses the first step in the de novo sphingolipid synthesis pathway. Previous studies have demonstrated that lace mutants have severely reduced sphingolipid content (Herr et al., 2003 Development 130, 2443-2453). Examination of the larval synapses of lace mutants identified aberrations in normal structure. While synapse length is normal, the number of boutons per synapse is reduced to $40 \%$ of the wild type number. Boutons are also larger with a high incidence of malformed terminal boutons with a 'spurlike' morphology. Ultrastructural analysis by electron microscopy reveals the active zones, T-bars and synaptic vesicle pool appear normal suggesting these boutons are functional. This is further supported by an electrophysiological examination, showing essentially intact synaptic transmission. However, disrupted membrane architecture is observed within the bouton, with enlarged vesicles, endosomes and grossly perturbed mitochondria. Such defined defects highlight a role for sphingolipids in the regulation of both synapse structure and mitochondrial dynamics.

V.13

Active zone macromolecular architecture in the mushroom body calyx of Drosophila Melanogaster

Frauke Christiansen-Engelhardt ${ }^{1}$, David Owald ${ }^{1}$, Florian Leiss $^{2}$, Wernher Fouquet ${ }^{2}$, Sara Mertel ${ }^{2}$, Stephan Sigrist

${ }^{1}$ Rudolf Virchow Center for experimental Biomedicine and Institut für klinische Neurobiologie, 97078 Würzburg, Germany

2 Max Planck Institute of Neurobiology, Am Klopferspitz 18, D-82152 Martinsried

Dynamic changes of both synapse structure and function are considered as essential substrates for learning and memory processes. Behavioral genetics in D. melanogaster could locate olfactory memory traces in the mushroom body kenyon cells (KCs) and in the projection neurons (PNs) that innervate the kenyon cells in the mushroom body calyx. While an insight into plasticity processes mediating olfactory learning at these synapses would thus be important, the molecular composition of the synapses and the connectivity within the calyx are up to now not known sufficiently.

Therefore, several transgenic pre- and postsynaptic markers, prominently BruchpilotGFP and the acetylcholine receptor subunit $\mathrm{D} \alpha 7 \mathrm{GFP}$, are used to visualize the population of the input and output synapses of KCs, PNs and GABAergic interneurons in the calyx. In parallel, antibody labeling against active zone proteins allows the identification of different synapse types. We also address the question whether $\mathrm{KC}$ neurites have exclusively axonal or dendritic character or if they can be of mixed type, and whether there might be microcircuits of KCs.

V.14

\section{The Fly Jump Muscle: From A to $W$ and back}

Chris Elliott $^{1}$, Julia Hill ${ }^{1}$, Adam Middleton ${ }^{1}$, Sean Sweeney ${ }^{1}$, John Sparrow

${ }^{1}$ Department of Biology, University of York

Flies jump by contracting the tergal depressor of trochanter (TDT, also known at TTM), to extend the mesothoracic femur and propel the fly upwards. We have constructed an ergometer to measure the output of this muscle. This allows us to examine the impact of development/aging and mutations affecting the NMJ on its functional output. 
Over the first 2 weeks of adult life, the TDT response to a single stimulus remains constant. By 3 weeks (when $80 \%$ of the flies are still alive) muscle output has dropped to $50 \%$ of the initial value. To test the effect of use on performance we maintained flies in petri dishes to prevent jumping, but their responses remained identical to flies kept in control conditions.

With repeated $3.5 \mathrm{~Hz}$ stimulation the TDT output declines. In 1 day old flies, it reaches $50 \%$ of the initial value after 30 stimuli. In older flies, or those with synaptic overgrowth mutations (highwire and spinster), performance declines more quickly.

Flies which cannot synthesise octopamine or tyramine produce only $60 \%$ of wild type TDT output. The importance of these amines is underscored by the dense aminergic innervation of both the giant TDT motoneuron and muscle. Aminergic fibres also entwine two additional motoneurons with restricted branching: electrophysiological recordings show these units are used during small leg movements (walking/grooming).

We conclude that although the jump circuit is known for its reliability, the neuromuscular link is fascinating in its diversity.

\section{V.15}

\section{Using STED microscopy to probe synapse architecture}

Wernher Fouquet ${ }^{l}$, David Owald ${ }^{1}$, Sara Mertel ${ }^{1}$, Robert Kellner ${ }^{2}$, Markus Dyba ${ }^{3}$, Stephan J. Sigrist ${ }^{3}$

${ }^{1}$ AG Sigrist, Bio-Imaging Center, Universität Würzburg ${ }^{2}$ AG Hell, MPI für Biophysikalische Chemie, Göttingen

${ }^{3}$ Leica Microsystems, Mannheim

The majority of rapid cell-to-cell communication mechanisms and information processing within the nervous system makes use of chemical synapses. Fast neurotransmission depends on an effective structural arrangement of cellular components on both sides of the synaptic cleft. The molecular organization of presynaptic active zones (AZs) during calcium influx-triggered neurotransmitter release is therefore a focus of intense investigation. We recently showed that Bruchpilot (BRP) of the Drosophila, which features homologies to the mammalian CAST/ERC family, is essential for structural organization and efficient neurotransmitter release. This work seeks to further explore the relation between synaptic architecture and vesicle release by using the fly NMJ as a model system, combined with the latest advances in fluorescence microscopy. The increased optical resolution, using STED technology, allows us to visualize complex inter- and intramolecular arrangements in synapses, which are unrecognisable in diffraction limited optical systems. These efforts are combined with observations achieved during live imaging experiments, meant to widen our understanding towards the protein dynamics, in order to gain further insights into the AZ architecture. Thus we want to shed light into whether the reorganization or impairment of the newly discovered AZ substructures are important for the proper neuronal function.

\section{V.17}

\section{Drosophila Cip4 couples membrane deformation and the actin cytoskeleton}

Robert Fricke ${ }^{1}$, Christina Gohl ${ }^{1}$, Astrid Grevelhörster ${ }^{1}$, Stefanie Wenderdel ${ }^{1}$, Christian Klämbt ${ }^{1}$, Sven Bogdan

${ }^{1}$ Institute of Neurobiology, University of Münster

A dynamic interplay between membrane systems and the actin cytoskeleton is essential for numerous aspects of nervous system function, such as the recycling of synaptic vesicles or growth cone movement. Members of the F-Bar protein family have been identified as proteins that couple membrane and cytoskeletal systems during endocytosis. Also, several of its members have already been shown to function in the nervous system, eg Nervous Wreck, which is important for NMJ development in Drosophila. To better undertstand the function of F-Bar proteins during Drosophila development, we initiated the characterization of the Drosophila Cip4 protein. Its domain structure comprises an $\mathrm{N}$ terminal F-Bar domain, that allows it to oligomerize and bind to the membrane and an $\mathrm{SH} 3$ domain that mediates binding to endocytotic proteins, such as Dynamin, or actin regulators, such as WASP/WAVE proteins. Our data suggest, that Drosophila Cip4 is able to induce invaginations of the plasma membrane to mediate endocytotic vesicle formation. It also seems to be able to recruit Dynamin to the site of endocytosis which then facilitates the scission of the vesicle. Furthermore, Cip4 is able to relocate WASP and WAVE to membrane invaginations, both of which mediate actin polymerization and are also necessary for nervous system development. Recently, we have been successful in generating a Cip4 loss-of-function mutant, which will now allow us to identify the cell biological processes that Cip4 is involved in.

V.18

Single-Cell Analysis of Innervation-Dependent Gene Expression during Synaptogenesis

Ai Fukui ${ }^{1}$, Gaku Tonoe ${ }^{1}$, Mikiko Inaki ${ }^{1}$, Hiroyuki Aburatani $^{2}$, Akinao Nose ${ }^{3}$ 
${ }^{1}$ Department of Physics, University of Tokyo

${ }^{2}$ Research Center of Advanced Science and Technology, University of Tokyo

${ }^{3}$ Department of Complexity Science and Engineering, University of Tokyo

Synapse is a specialized site of cell-cell contact where various molecules are localized for synaptic transmission. Reciprocal induction between pre- and postsynaptic cells is believed to be important for the differentiation of the synaptic structures. The induction process likely involves regulation of gene transcription, yet the identity and function of genes that are induced during this process remain largely unknown. Here, we aim to investigate the mechanisms of how innervation-dependent gene expression in the postsynaptic cell supports synaptic differentiation, using the neuromuscular system in Drosophila. We performed genome-wide DNA microarray analysis of individually isolated muscle cells to detect changes of gene expression in the postsynaptic cells. By comparing expression profiles of muscles before and after innervation and muscles in mutants that lack innervation, we identified 82 candidate genes that are potentially regulated by nerve innervation. For some of these genes, the changes of expression level were confirmed by quantitative PCR. These identified genes included extracellular matrix components, transcription factors and various enzymes. We are now performing genetic analyses of these genes to elucidate their roles in synaptic differentiation.

V.19

Proteins of the Presynaptic Cytoskeleton Mediating Synaptic Stability at the Drosophila NMJ

Bernd Goellner ${ }^{1}$, Daniel Banovic ${ }^{1}$, Matthias Siebert ${ }^{1}$, Hermann Aberle ${ }^{1}$

1 Institut für Neurobiologie, Westfälische Wilhelms Universität Münster

Transsynaptic adhesion sites are linked to the underlying cytoskeleton by a large scaffold of specalized proteins forming a synaptic density. Its disruption causes synaptic destabilization accompanied by synapse retraction -an early feature in the pathogenesis of many neurodegenerative diseases. At the Drosophila neuromuscular junction Ankyrin2 stabilizes synapses by connecting two major parts of the presynaptic cytoskeleton: the actin/spectrinbased membrane skeleton and the tubulin-based core cytoskeleton. To examine the role of cytoskeletal proteins on synaptic stability, we performed a survey of candidate genes using transgenic RNAi lines. In addition, we will present the results of an unbiased RNAi screen specifically designed to identify presynaptic regulators of synaptic stability.

V.20

Wave/Scar has a function in photoreceptor axon targeting in Drosophila

Christina Gohl ${ }^{l}$, Raiko Stephan ${ }^{l}$, Christian Klämbt ${ }^{1}$, Sven Bogdan ${ }^{1}$

${ }^{1}$ Institute for Neurobiology, University of Münster

Actin assembly and dynamics are vitally important for the directed motility of diverse cell types, for example proper growth cone migration during nervous system development. The members of the Wiskott-Aldrich-Syndrome family, Wasp and Wave/Scar, are regulators of actin dynamics. Although both Wasp and Wave activate the Arp2/3-complex, a prominent actin nucleator, only Wave is essential for the correct projection of photoreceptor axons in the optic lobe during Drosophila development.

Wave is part of a protein complex, the so called Wave complex, also containing the Abl interactor (Abi) protein, which is necessary for proper photoreceptor targeting as well. A similar phenotype as for wave and abi was reported for mutations in the dreadlocks (dock) gene, which encodes a $\mathrm{SH} / \mathrm{SH} 3$ adaptor protein. Abi binds to the N-terminal SH3-domain of Dock and might thereby link the adaptor protein Dock to the actin regulator Wave.

V.21

Circadian expression of the presynaptic active zone protein Bruchpilot in the lamina of Drosophila melanogaster.

Jolanta Górska-Andrzejak ${ }^{1}$, Ewelina Niañko ${ }^{1}$, El_bieta Pyza ${ }^{l}$

${ }^{1}$ Cytology and Histology, Jagiellonian University, Krakow, Poland

In the first optic neuropil (lamina) of the fly's visual system the morphology of cells and the number of synapses change during the day and night. In the present study we examined the expression of a presynaptic protein Bruchpilot (BRP) in the lamina synaptic units, so called cartridges, using Mab nc82 (Hybridoma). Wildtype Canton-S, arrhythmic per ${ }^{01}$ mutants and transgenic GAL4/UAS lines of Drosophila were held in light/dark (LD 12:12) or in constant darkness (DD) conditions, decapitated at different times of the day and processed for confocal microscopy. We observed a characteristic 
spot-like pattern of BRP expression within the cartridge with the majority of nc82-immunoreactive sites localized to photoreceptor terminals (R1-R6). Intensity of fluorescence and the number of fluorescent spots at different times in LD showed clear daily oscillations. Both the abundance of BRP and the number of synapses in the lamina cartridge were the highest one hour after lights-on (ZT1), and the lowest three hours later (ZT 4). Such oscillations were not observed in DD. In per $^{01}$ mutants, however, neither in LD nor in DD significant changes in the level of fluorescence and the number of fluorescent spots could be detected. Our results indicate that the daily rhythm in BRP expression correlates with circadian changes in the length of dendrites of the postsynaptic L2 monopolar cell, and is controlled by a circadian clock, even though it is not maintained in DD.

\section{V.22}

\section{Identification and characterization of brain proteins in} Drosophila melanogaster.

Partho Halder $^{1}$, Alois Hofbauer ${ }^{2}$, Erich Buchner ${ }^{1}$

${ }^{1}$ Genetics \& Neurobiology, Universität Würzburg

${ }^{2}$ Zoology, Universität Regensburg

We aim to identify proteins from Drosophila melanogaster brain and study their function. Two of the monoclonal antibodies, raised against fruit fly brain homogenate (Hofbauer 1991) gave clear signal on western blots. (na21, ab52) produced distinct signals on western blots of fly head extracts. na21 recognizes an antigen with $\mathrm{M}_{\mathrm{r}} \sim 10 \mathrm{kD}$, while ab52 recognizes an antigen with $\mathrm{M}_{\mathrm{r}} \sim 95 \mathrm{kD}$. On cryosections na21 stains specific synaptic layers of the optic neuropil, like the most proximal layer of medulla and parts of lobula and lobula plate. It also stains sensory cells in the antennae, central brain regions, thoracic ganglion and a pair of organs near the tip of the abdomen. ab52 apparently stains the entire synaptic neuropil of the brain. nb181 seems to be eye-specific as it stains only eyes and photoreceptor terminals in the lamina and medulla. Ultracentrifugation of fly head lysates at $100000 \mathrm{~g}$ indicate that the antigen for na21 remains in the pellet, from which it is not completely extracted by $\mathrm{Na}_{2} \mathrm{CO}_{3}$ treatment, hence it seems to be a membrane protein. The antigen for ab52 is present in the supernatant, thus it is a soluble (cytosolic) protein. We are currently trying to identify the antigens of na21, ab52 \& nb181 using various techniques like immunoprecipitation, TricineSDS-PAGE, 2DGE, followed by MS. Upon identification, we will then try to generate mutant lines for the gene and study the various phenotypes of the mutants in order to elucidate the function of the protein.
V.23

Regulation of molecular architectures of type 1 glutamatergic synapses in drosophila larval neuromuscular junctions by fragile $\mathrm{x}$ mental retardation protein

Thi Thu Cuc Ho ${ }^{1}$, Dae-Weon Lee ${ }^{1}$, Young Ho Koh ${ }^{1}$

${ }^{1}$ Biomedical Gerontology Program, ILSONG Institute of Life Science Hallym University

Fragile $\mathrm{X}$ mental retardation syndrome has been shown to be caused by the loss of fragile $\mathrm{X}$ mental retardation protein (FMRP). Since molecular components of Drosophila Type 1 glutamatergic synapses in larval neuromuscular junctions are similar to those of excitatory synapses in human central nervous system, we are utilizing Drosophila Type 1 synapses as a model to study how loss of FMRP at synapses alter molecular architectures at the glutamatergic synapses. Consistent with previous studies, we found that loss of FMRP induced altered NMJ morphologies. In addition, the localization of Discs Large, a Drosophila homologue of PSD 95/SAP 90, was significantly reduced at the postsynaptic terminals. These results suggested that FMRP may play important roles in differentiation of synaptic architecture in Drosophila larval glutamatergic synapses by regulating expression or localization of DLG.

Key words: Fragile X syndrome, FMRP, Discs large, mental retardation, synapses

V.25

fluglotse is required for synapse maturation at the Drosophila neuromuscular junction

Jeannine Kern ${ }^{1}$, Stella Kramer ${ }^{1}$, Petra Füger ${ }^{1}$, Tamara Finster $^{1}$, Natalia Hallay ${ }^{1}$, Tobias M Rasse $e^{l}$

${ }^{1}$ Department of Cellular Neurology, Hertie Institute for Clinical Brain Research, University of Tuebingen

Synapse formation involves bidirectional signaling between pre- and postsynaptic cells leading to the development of specialized structures for release and detection of neurotransmitters. Following formation and initial maturation more than $95 \%$ of all individual synapses will be stabilized at Drosophila melanogaster neuromuscular junctions. We thus used the presence of pre- and postsynaptic marker proteins -Bruchpilot and GluRIIIto screen for mutants in which synapses are not correctly stabilized. Here, we describe one of the identified mutants: fluglotse. Homozygous fluglotse mutant larvae are characterized by impairments in axonal transport, 
defects in synaptic structure and function as well as by impaired locomotion. About $30 \%$ of all synapses on muscle $6 / 7$ are devoid of presynaptic Bruchpilot staining. Behavioral assays indicate that defects are caused by impaired synapse assembly rather than by defects in the stabilization of synapses. The temporal sequence of molecular and cellular events that lead to the formation of these "defective" synapses was clarified using in vivo imaging.

\section{V.26}

The in vivo functions of heat shock cognate proteins in regulating synaptic plasticity in Drosophila larval neuromuscular junctions

A Young Kim ${ }^{1}$, Won Tae Kim, Dae Weon Lee, Young Ho Koh

${ }^{1}$ Molecular Neurogenetics Lab., Ilsong Institute of Life Science, Hallym University

Molecular chaperones are major regulators of formation, trafficking, and/or degradation of cellular proteins. Recent studies in the functions of molecular chaperones at neurons and synapses suggested that variety of molecular chaperones may play pivotal roles in regulating in synaptic plasticity. We have been utilizing Type 1 glutamatergic synapses in Drosophila larval neuromuscular junctions (NMJs) to study unknown functions of molecular chaperones in synaptic plasticity. Recently, we identified a temperature sensitive paralytic behavioral mutant fly had a molecular lesion at Heat Shock Cognate Protein 70-3. We found that the mutant alleles of HSC 70-3 showed altered synaptic morphology. These data suggested that HSC70-3 is important for maintaining normal synaptic functions and structures. Further studies in HSC 70-3 mutant flies we have identify will reveal unknown roles of molecular chaperones in regulating synapse development and plasticity.

Key words: Synaptic plasticity, molecular chaperone, DLG, Dpak

V.27

Requirement of presynaptic proteins for specific components of associative memory

Stephan Knapek ${ }^{1}$, Eva Dierich-Schmitt ${ }^{2}$, Bertram Gerber $^{2}$, Stephan Sigrist ${ }^{2}$, Erich Buchner ${ }^{2}$, Martin Heisenberg $^{2}$, Hiromu Tanimoto ${ }^{1}$
${ }^{1}$ Max Planck Institute of Neurobiology

${ }^{2}$ University of Würzburg

To understand the synaptic plasticity underlying learning, we examined olfactory associative memory. In this paradigm, the flies associate an odor with electric shock punishment. Here we focus on Synapsin, an abundant presynaptic protein regulating the vesicle release under high frequency stimulation. The null mutant for synapsin shows significant deficits in short-term memory. This memory phenotype is most prominent within the first few hours after training. We found that Synapsin is selectively required for anaesthesia-sensitive memory which is a labile memory component decaying quickly after training. In contrast, a longer-lasting memory does not require Synapsin. As the response to the employed odors and the electric shock is still intact, our results suggest that Synapsin-dependent vesicle release controls short-term memory. Furthermore, we found that Bruchpilot, another presynaptic protein specifically localized to the active zones, affects a complementary memory component (anaesthesia-resistant memory). Thus, these two forms of memory might be generated in parallel by two different presynaptic mechanisms.

V.28

The DlgS97-Metro-DLin-7-scaffold complex is required for scaling and spacing of glutamate receptor fields at larval NMJs

Oliver Kobler ${ }^{1}$, Andre' Bachmann ${ }^{2}$, Carolin Wichmann ${ }^{3}$, Robert Kittel ${ }^{4}$, Stephan J. Sigrist ${ }^{4}$, Elisabeth Knust ${ }^{5}$, Jimena Sierralta ${ }^{6}$, Eckart D. Gundelfinger ${ }^{1}$, Ulrich Thomas $^{1}$

${ }^{1}$ Leibniz Institut für Neurobiologie, Magdeburg

2 Insitut für Genetik, Uni Düsseldorf

${ }^{3}$ European Neuroscience Institute, Göttingen

${ }^{4}$ Rudolf-Virchow-Zentrum, Uni Würzburg

5 MPI für molekulare Zellbiologie und Genetik, Dresden

6 Instituto de Ciencias Biomedicas, Universidad de Santiago de Chile

Dlg, a prototypic MAGUK serves as a scaffold molecule at larval NMJs. It ensures proper localization of $\mathrm{K}^{+}$channels, FasII and of secondary scaffold proteins such as Scribble, Metro and DLin-7. Loss of Dlg affects synaptic bouton morphology and leads to expansion of active zones and glutamate receptor fields. We have found that two major subclasses of Dlg, DlgA and DlgS97, are expressed at NMJs, suggesting the co-existence of separate Dlg-based complexes. The postsynaptic recruitment of DLin-7 and Metro largely depends on DlgS97, with Metro, 
an MPP-like MAGUK, providing a physical link between DlgS97 and DLin-7. The relationship between all three proteins is bidirectional, though to various extent. Metro is required for recruitment of Dlin-7 and in turn remains undetectable in $d$ Lin-7 mutants. $\mathrm{DlgS} 97$ but not $\mathrm{DlgA}$ is reduced by about $40 \%$ in metro mutants. Like in dlgS97 mutants a reduction in bouton number and a loosely packed SSR is also observable. EM-analyses and 3D imaging reveal irregular spacing and a broadened size distribution of receptor fields. The number of synaptic contacts is moderately altered and basal synaptic function appears unaffected. Interestingly, muscle expression of active CaMKII causes a severe reduction of DLin-7 despite the persistence of considerable amounts of DlgS97. This suggests that the trimeric scaffold complex is subject to an activity-dependent regulation that controls the microarchitecture of synaptic and perisynaptic compartments.

V.29

Dynamisms of postsynaptic filopodia during target recognition of Drosophila Neuromuscular Junction

Hiroshi Kohsakal, Akinao Nose ${ }^{2}$

${ }^{1}$ Department of physics, Graduate School of Science, University of Tokyo

2 Department of Complexity Science and Engineering, Graduate School of Frontier Sciences, The University of Tokyo

For wiring a functional nervous system, precise partner recognition between axons and their target cells is essential. Dynamisms of presynaptic growth cones have been regarded as a major mechanism for neuronal matchmaking. Recent live-imaging studies, however, show the dynamic nature of postsynaptic cells during targeting, which suggests that postsynaptic dynamism may also contribute to target recognition processes. Here we studied target recognition by myopodia, postsynaptic filopodia in muscles during the formation of neuromuscular connectivity. By in vivo time-lapse imaging, we found that initial neuromuscular contacts occur at the tips of myopodia. Some contacts are stabilized to form synapse, whereas others are eventually eliminated. The fraction of stabilized contacts greatly differs depending on whether the contact is made with partner or non-partner motor neurons. These data collectively suggest that postsynaptic filopodia are able to recognize the appropriate synaptic partners. Previous genetic analysis implicated that cell adhesion molecule Capricious (CAPS) as an attractive target recognition molecule on muscle12 (M12). We found that CAPS concentrates at the tips of myopodia. In caps mutants, the number of stabilized contacts between M12 and the innervating motor neurons is reduced. These findings are consistent with the notion that local, contactmediated signaling at the tips of postsynaptic filopodia is crucial for target selection.

V.30

Functional requirements of L1-type cell adhesion molecule Neuroglian and its interaction with Semaphorin 1a in circuitry formation

Shirisha Kudumala ${ }^{1}$, Monica Mejia ${ }^{1}$, Rod Murphey ${ }^{1}$, Tanja Godenschwege ${ }^{l}$

${ }^{1}$ Biological Sciences, Florida Atlantic Unversity

The giant fiber (GF) system is the neuronal circuit that elicits the escape response of the fly. The giant fibers receive visual input from the optic lobes. Each GF extends a large axon from the brain into the second thoracic neuromere where it grows a large presynaptic terminal along the medial dendrite of its respective tergotrochanteral motor neuron (TTMn). We study the molecules and signaling pathways that are involved in the assembly of this central synapse between the giant fiber and the jump motor neuron. We have previously demonstrated a function for Neuroglian and Semaphorin1a in Drosophila giant fiber circuit formation. Both molecules are required for guiding the giant fibers out of the brain and have distinct functions during giant synapse formation. Here, we further characterize the functional requirements of the intra- and extracellular domains of Nrg/L1CAM in giant synapse formation and its interaction with Semaphorin 1a in circuit formation. We found that Neuroglian and Semaphorin1a genetically interact with each other during axon guidance as well as during synapse formation. In addition, our findings suggest that Neuroglian and Semaphorin1a interact in cis to regulate each other's functions with different signaling outputs during guidance and synapse formation.

V.31

Nervous wreck and Synapsin-Dependent Regulation of Synaptic Vesicle Distribution at Active Zones

Dae-Weon Lee ${ }^{1}$, Sang-Hee Lee ${ }^{2}$, YoonJung Kim ${ }^{1}$, YongSun Kim ${ }^{1}$, Young Ho Koh ${ }^{1}$

${ }^{1}$ ILSONG Institute of Life Science, Hallym University, Anyang, Korea

${ }^{2}$ High Voltage Electron Microscopy Team, Korea Basic Research Institute, DaeJeon, Korea

Nervous Wreck (NWK), a SH3 domain and FCH motive protein present at Type 1 glutamatergic synapses as 
reticular structures is a Drosophila homolog of human srGAP2 protein, linked to X-linked mental retardation. Circles in NWK reticulum enclosed T-shaped active zones and partially co-localized with synaptic vesicle markers or exo/endocytosis components at confocal immuno-fluorescence microscopy. At Electorn microscopy (EM) levels, NWK proteins localized at the edge of synapses and synaptic vesicle pools. Synaptic architectures and sizes in $n w k$ null mutant NMJs were significantly different from those of wild-type control NMJs. In addition, 3 dimensional High-Voltage EM tomography analysis proved that the sizes and the densities of synaptic vesicles in $n w k$ null mutant synapses were altered. Furthermore, anti-Drosophila Synapsin antibodies co-immunoprecipitated NWK. Taken together, NWK is important for maintaining synaptic architectures and SV sizes and pools at T-shaped active zones by interacting with Synapsin.

Keywords: Nervous Wreck, glutamatergic synapses, Synapsin, synaptic architectures

\section{V.33}

\section{A peripheral pacemaker seem to drive the rhythm in bouton size}

Kerstin I. Mehnert ${ }^{1}$, Rafael Cantera ${ }^{1}$

${ }^{1}$ Zoology, Stockholm University

Circadian rhythms in the morphology of neurons have been demonstrated in the fly Drosophila melanogaster. One such rhythm is characterized by changes in the size of synaptic boutons of an identified flight motor neuron, with larger boutons during the day compared to the night. A more detailed temporal resolution of this rhythm shows here that boutons grow at a time of increased locomotor activity during the morning, but become gradually smaller during the day and second period of increased locomotoractivity in the evening. We experimentally manipulated the fly's synaptic activity during short periods of the day to investigate if changes in bouton size could be a consequence of the different levels of synaptic activity associated with the fly's locomotion rhythm. In the late night and early morning, when the flies normally have an intense period of locomotion, the boutons grew independently of whether the flies were active or completely paralyzed. Bouton size was not affected by sleepdeprivation during the early night. The cycle in bouton size persisted during two days even in decapitated flies, which do not move, reinforcing the notion that it is largely independent of synaptic activity and showing that a pacemaker other than the main biological clock can drive it.
V.34

Acute photoinactivation of Clathrin Heavy Chain inhibits synaptic recycling but allows bulk membrane uptake upon stimulation

Katarzyna Miskiewicz ${ }^{1}$, Jaroslaw Kasprowicz ${ }^{1}$, Sabine Kuenen $^{1}$, Patrik Verstreken ${ }^{1}$

${ }^{1}$ Department of Molecular and Developmental Genetics, Center for Human Genetics, VIB, K.U.Leuven

Clathrin-mediated endocytosis is critical to maintain release during intense neuronal activity; however, the exact function of clathrin during vesicle formation remains enigmatic. We have therefore investigated role of clathrin at the synapse using fly mutants, pharmacology and acute photoinactivation of chc. Like other endocytic Drosophila mutants, synapses that lack functional chc fail to maintain neurotransmission during intense stimulation. Conversely, chc mutant synapses take up massive amounts of membrane upon stimulation as gauged by FM 1-43 labeling. Furthermore, electron microscopy shows a clear decrease in synaptic vesicle number and the formation of giant $(>1 \mu \mathrm{m})$ membrane invaginations. These membranes fail to recycle and be resolved in individual small synaptic vesicles. Unlike synapses that lack functional chc, Drosophila mutants that affect dynamin function, including shibire ${ }^{t s 1}$, synaptojanin or dap160 show a dramatic reduction or even loss of FM 1-43 dye uptake at the synapse. Interestingly, additional removal of chc in these mutants now allows for massive membrane uptake, suggesting clathrin may coordinate with dynamin to form synaptic vesicles. Our data point to a critical role for chc during synaptic vesicle recycling, and reveal a form of bulk membrane retrieval that induces membrane internalization in the absence of chc.

V.35

\section{Analysis of Synapsin, SAP47 and TBCE-like function in Drosophila melanogaster}

Tulip Nuwal ${ }^{1}$, Erich Buchner ${ }^{1}$

${ }^{1}$ Genetics and Neurobiology, University of Wuerzburg

Synapsins are neuron-specific phosphoproteins widely distributed on the surface of synaptic vesicles. SAP47 (Synapse associated protein of $47 \mathrm{kDa}$ ) is also vesicle associated in Drosophila. In Drosophila it is observed that $S y n^{97}$ and $S_{a p 47^{156}}$ null mutants have defects in learning and memory (cf. Michels. B. et al., Saumweber. T. et al., this volume) but have no remarkable defects in viability or fertility. Western blots of brain homogenates 
from $\operatorname{Sap} 47^{156}$ null mutants show an additional synapsin signal in comparison to wild-type (CS) which is lost by treatment of the homogenate with phosphatase. On protein quantification by ELISA, Sap $47^{156}$ null mutant flies showed a 2.7 fold increase in synapsin content compared to CS flies. Thus these results hint towards a possible interaction between SAP47 and synapsin. To investigate the possibility of a direct molecular interaction between SAP47 and synapsin, Co-IP experiments are currently being performed. Homozygous double mutants of $S a p 47^{156}$ and $S y n^{97}$ are being analyzed for defects in viability, fertility and in various behavioral assays.

Vertebrate Tubulin specific chaperone E (TBCE) is implicated in microtubule assembly and a missense mutation in the mouse TBCE gene causes progressive motor neuronopathy (pmn) which serves as a model for spinal muscular atrophy (SMA). A yeast two hybrid screen revealed a TBCE-like protein as an interacting partner of SAP47. Currently, antisera against the Drosophila homolog of TBCE-like (CG12214) protein are generated.

V.36

The role of Sphingolipid in neuronal structure and function, with insight into a human neuropathy

Matthew Oswald ${ }^{1}$

${ }^{1}$ The University of York

Sphingolipids are potent signalling molecules and ubiquitous components of the plasma membrane. Sphingolipids make up $10 \%$ of all lipids in neuronal tissue, where their importance in neuronal function is highlighted by multiple sphingolipid related neuropathies. Sphingolipids have long been known to be enriched at synapses, however their function there has not been defined. Here, mutants in Serine Palmitoyltransferase (SPT) the enzyme responsible for the initial step in de-novo sphingolipid synthesis have been used to generate a sphingolipid deficient fly. We are using these flies to explore the role of sphingolipids in neuromuscular junction development and to model a human neuropathy, Hereditary Sensory and Autonomic Neuropathy type I (HSAN1), which is caused by SPT dysfunction. We have generated an SPT dominant negative transgene based upon the most common mutation known to cause HSAN1. Using this transgene to selectively reduce sphingolipid biogenesis we have demonstrated that synaptic development requires both pre- and post-synaptic sphingolipid availability. Reducing sphingolipid synthesis in the peripheral nervous system decreases larval sensitivity to noxious heat stimuli in concordance with the sensory deficit observed in HSAN1. In this manner we will ascertain the role of sphingolipid in synaptic and dendritic development and function.

V.37

\section{Analysis of the active zone protein DSyd1}

David Owald $^{l}$, Wernher Fouquet ${ }^{1}$, Manuela Schmidt ${ }^{1}$, Carolin Wichmann ${ }^{1}$, Frauke Christiansen ${ }^{1}$, Harald Depner $^{1}$, Jorg Körner ${ }^{1}$, Sara Mertel ${ }^{1}$, Stephan J Sigrist ${ }^{1}$

${ }^{1}$ Rudolf Virchow Center, University of Würzburg

We recently showed that after loss of Bruchpilot (BRP, related to the mammalian CAST family of active zone proteins), active zone electron-dense projections (T-bars) were entirely lost, $\mathrm{Ca}^{2+}$ channels were reduced in density, evoked vesicle release was depressed, and short-term plasticity was altered (Kittel et al 2006). We identified the Drosophila Syd1 homologue (DSyd1) as direct binding partner of BRP taking an unbiased proteomics approach. DSyd1 localizes to active zones both at the larval neuromuscular junction (NMJ) and at central synapses. Here we present light microscopic, ultrastructural and electrophysiological analysis of $d s y d l$ mutant larvae.

V.38

The role of Dystrophin at Drosophila synapses

Gonneke Pilgram ${ }^{1}$, Richard Baines ${ }^{2}$, Lee Fradkin ${ }^{1}$, Jasprien Noordermeer ${ }^{1}$

1 Laboratory of Developmental Neurobiology, Dept. Molecular Cell biology, Leiden University Medical Centre

${ }^{2}$ Faculty of Life Sciences, University of Manchester

Duchenne Muscular Dystrophy is a fatal genetic disorder caused by mutations in the dystrophin gene and is characterized by progressive muscle wasting. Dystrophin is required for muscle stabilization during contraction and as a scaffold for several signaling pathways. A specific set of mutations in dystrophin is correlated with cognitive defects, indicating its importance in mammalian brain. Little is known, however, about its function in the brain and no therapy is available to treat the mental and muscular deficits. Therefore, it is important to get more insight into initial cellular changes arising in the absence of different Dystrophin isoforms. The dystrophin gene is conserved in Drosophila melanogaster and encodes long and short isoforms. We found that reduction of a short 
muscle specific isoform Dp117 results in progressive muscle degeneration in larvae and adult flies. Using electrophysiology, we demonstrated that lack of the short brain specific isoform Dp186, or the long muscle specific isoform DLP2, results in increased neurotransmitter release in the neuropile and at the neuromuscular junction (NMJ), respectively. Rescue and RNA-interference experiments indicate that dystrophin functions primarily postsynaptically at both synapses. These data suggest a new role for Dystrophin at synapses in regulating or maintaining retrograde signaling. Currently, we study the involvement of BMP-pathway members and other synaptic proteins in Dystrophin function at the NMJ and CNS.

\section{V.39}

\section{Functional Analysis of Dystrobrevin during Droso- phila Development}

Saranyapin Potikanond ${ }^{1}$, Mariska C. van der Plas ${ }^{1}$, Anja W.M. de Jong ${ }^{1}$, Lee G. Fradkin ${ }^{1}$,

Jasprina N. Noordermeer ${ }^{1}$

${ }^{1}$ Laboratory of Developmental Neurobiology, molecular cell biology, Leiden University Medical center

Dystrobrevin is a component of the dystrophin-glycoprotein complex (DGC). Mutations in many of the genes encoding DGC members can cause muscular dystrophies. Most members of the DGC are also found at the neuromuscular junction (NMJ), where their deficiency is often associated with NMJ structural defects. Hence, synaptic dysfunction may also contribute to the pathology of dystrophic muscles. Mutations in dystrobrevin are associated in humans with congenital heart disease, although it is unknown if muscle defects underlie the cardiac dysfunction. In mice, lack of Dystrobrevin causes a mild muscular dystrophy. Here, we show that Drosophila Dystrobrevin is expressed at the neuromuscular junction, at the sarcomeric I-band in the muscle and in the neuropile and brain. Furthermore, we find that Dystrobrevin co-localizes with Dystrophin, another member of the DGC, at the NMJ and in the CNS and that Dystrobrevin is delocalized in dystrophin mutants. To examine the effects of reduced or absence of expression of Dystrobrevin, we are analyzing transgenic flies that express double-stranded dystrobrevin RNA in specific domains and are in the process of making dystrobrevin loss-of function mutants by homologous recombination. Our preliminary evidence suggests roles for dystrobrevin in synaptic transmission and in maintaining the integrity of the muscle.
V.41

\section{A Glial Variant of the Vesicular Monoamine Trans- porter is Required to Store Histamine in the Droso- phila Visual System}

Rafael Romero-Calderón ${ }^{1}$, Guido Uhlenbrock ${ }^{2}$, Jolanta Borycz $^{3}$, Anne F. Simon ${ }^{1}$, Anna Grygoruk ${ }^{1}$, Susan K. Yee $^{1}$, Amy Shyer ${ }^{1}$, Larry C. Ackerson ${ }^{4}$, Nigel T. Maidment $^{4}$, Ian A. Meinertzhagen ${ }^{3}$, Bernhard T. Hovemann $^{2}$, David E. Krantz ${ }^{1}$

${ }^{1}$ Gonda Center for Neuroscience and Genetics Research, Los Angeles, CA, USA

${ }^{2}$ Ruhr-Universität Bochum, Fakultät für Chemie und Biochemie, Bochum, Germany

${ }^{3}$ Life Sciences Centre, Dalhousie University, Halifax, Nova Scotia, Canada

${ }^{4}$ Hatos Center for Neuropharmacology, Los Angeles, CA, USA

Unlike other monoamine neurotransmitters, the mechanism by which the brain's histamine content is regulated remains unclear. In mammals, vesicular monoamine transporters (VMATs) are expressed exclusively in neurons and mediate the storage of histamine and other monoamines. We have studied the visual system of Drosophila melanogaster in which histamine is the primary neurotransmitter released from photoreceptor cells. We report here that a novel mRNA splice variant of Drosophila VMAT (DVMAT-B) is expressed not in neurons but a small subset of glia in the lamina of the fly's optic lobe. Histamine contents are reduced by mutation of $d V M A T$, but can be restored by specifically expressing DVMAT-B in glia. Our results indicate a novel function for a vesicular monoamine transporter in glia that may be relevant to histamine homeostasis in other systems.

V.42

Anatomy of glutamatergic processes in the Drosophila larval nervous system.

Mai Saito ${ }^{1}$, Irina Sinakevitch ${ }^{1}$, Thomas Rival ${ }^{1}$, Magali Iché $^{1}$, Serge Birman ${ }^{1}$

${ }^{1}$ IBDML-Developmental Biology Institute of MarseilleLuminy, CNRS-Université de la Méditerranée

L-glutamate (Glu) is the excitatory neurotransmitter at insect neuromuscular junctions (NMJ). However, little is known about the precise localization and physiological roles of glutamatergic interneurons in the Drosophila 
central nervous system. The vesicular Glu transporter (dVGluT) concentrates Glu into synaptic vesicles of glutamatergic neurons. In order to visualize these cells, we compared Glu-like, dVGluT and GFP immunoreactivities in driver lines expressing either n-synaptobrevinGFP (n-syb-GFP) (addressed to cell bodies, axons and terminals) or dVGluT-GFP (addressed to presynaptic sites). We compared two driver lines: OK371-GAL4, an enhancer trap inserted close to dVGluT, and dVGluTGAL4, transformed with a transgene containing $5.5 \mathrm{~kb}$ of DVGluT regulatory DNA. Glutamatergic processes were observed at the NMJ and in the ventral nerve cord and brain. All major neuropils of the larval brain (mushroom body and antennal lobes, lateral protocerebrum, visual neuropil, tritocerebrum and subesophageal ganglion) receive abundant glutamatergic innervation, suggesting a role of Glu in a large range of larval behaviors. GFPpositive neurons were less numerous in dVGluT-GAL4 than in OK371-GAL4 but always co-localized with Glulike and dVGluT immunoreactivities. In addition, the patterns of these drivers appeared somewhat complementary, in particular at the NMJ. Combination of these two drivers yielded a double GAL4 line targeting the whole glutamatergic system.

\section{V.43}

Social isolation enhances larval motoneuron excitability and adult female aggression: Effects of mutations altering cellular ROS levels.

\author{
Atsushi Ueda ${ }^{1}$, Chun-Fang $W u^{1}$ \\ ${ }^{1}$ Department of Biology, University of Iowa
}

Social deprivation is known to trigger a variety of behavioral and physiological changes in many animal species, but the underlying genetic and cellular mechanisms are not fully understood. As we reported previously, adult female flies reared in isolation after eclosion show higher frequency of aggressive behaviors than those reared in group. In this study, we found markedly enhanced activity-dependent facilitation of synaptic transmission at larval neuromuscular junctions when larvae were reared in isolation. Upon repetitive stimulation, group-reared larvae normally displayed a gradual increase in synaptic transmission, whereas a large fraction of isolation-reared larvae showed an explosive increase in the amount of transmitter release coupled with supernumerary firing of motor axons. We report that mutations of two genes, Hyperkinetic (Hk) and glutathione S-transferase-S1 (gsts1) alter the response to social isolation. $\mathrm{Hk}$ and gsts1 mutants displayed increased adult female aggression and larval neuromuscular hyperexcitability even when reared in group. Significantly, these mutant phenotypes were not enhanced further by isolation rearing. Mutations of these two genes are implicated in reactive oxygen species (ROS) metabolism and have been shown to alter neuronal ROS levels at neuromuscular junctions. Our data suggest potential involvement of ROS regulation in the cellular processes of neuronal hyperexcitability and aggressive behaviors induced by social isolation.

V.44

A novel X-chromosome EMS screen identifies genes implicated in neuronal communication

Valerie Uytterhoeven ${ }^{l}$, Jaroslaw Kasprowicz ${ }^{1}$, Sabine Kuenen ${ }^{1}$, Bassem Hassan ${ }^{1}$, Patrik Verstreken ${ }^{1}$

${ }^{1}$ VIB, Dept. of Molecular and Developmental Genetics, University of Leuven, Center for Human Genetics

In several neurological diseases, altered synaptic communication is thought to be one of the leading causes. We performed a genetic screen to identify novel mutations on the $\mathrm{X}$ chromosome affecting synaptic communication. Our strategy involves a three generation screen where we first create flies with random mutations, we then isogenize $\mathrm{X}$ chromosomes and finally we determine lethality in hemizygous males. As we surmise neuro-communication to be essential for life, only stocks with a lethal $\mathrm{X}$ chromosomes are maintained. To test for defective neuronal communication, we created female flies homozygous for the mutagenized $\mathrm{X}$ chromosomes only in their eyes and recorded electro-retinograms (ERGs) from these mutant eyes. Thus far, about 1,200 F2 stocks were isogenized and approximately 500 carry lethal X linked mutations; 30 of these show aberrant on or off transients in ERGs. Our goal is to isolate 200 mutant stocks by screening about 8,000 lethals. To distinguish between mutants that affect synaptic function and axonal development, we will also label the membranes of the mutant photoreceptors with antibodies to reveal their projection pattern. Mutants with abnormal ERGs but proper photoreceptor connections are good candidates for defects in neuronal communication. These mutants will be pursued further for mapping experiments using $\mathrm{X}$ chromosome duplications and high throughput sequencing. Finally, we will further characterise the mutants using electrophysiology and live imaging.

V.45

Pathways of $\beta$-alanine supply and their significance for vision in Drosophila.

Anna Ziegler ${ }^{1}$, Jens Rister $^{2}$, Martin Heisenberg ${ }^{2}$, Bernhard Hovemann ${ }^{1}$ 
1 Ruhr-Universität Bochum, Fakultät für Chemie und Biochemie, AG Molekulare Zellbiochemie, Universitätsstraße 150, 44780 Bochum, Germany

2 Biozentrum, Universität Würzburg, Lehrstuhl für $\mathrm{Ge}$ netik und Neurobiologie, Am Hubland, 97074 Würzburg, Germany

The neurotransmitter histamine is cycling between photoreceptor axonal endings and epithelial glia in Drosophila eyes. Photoreceptor expressed Tan, a $\beta$-alanyl-histamine hydrolase, and glial expressed Ebony, a $\beta$-alanyl-histamine synthase, are components of the underlying biochemical pathway. In this pathway, histamine is first converted by Ebony to its inactive form, carcinine ( $\beta$ alanyl-histamine) and then set free by hydrolysis to histamine and $\beta$-alanine by Tan. Visual capacity can be measured with electroretinographic (ERG) measurements, in which ON- and OFF-transients are indicators for signal transmission from photoreceptors to postsynaptic lamina cells. These ERG components are missing in ebony and tan mutant flies revealing a disrupted signal transmission.

The L-aspartate- $\beta$-decarboxylase Black has been claimed to be the $\beta$-alanine provider for Ebony in epithelial glia. Therefore, the black mutation should also give rise to missing $\mathrm{ON}$ - and OFF-transients. Surprisingly, the latter are wild type in black mutants. Behavioral tests, however, revealed effects of the mutation on some visually guided behaviors. While black flies had a wild type optomotor response even in the vicinity of the low intensity threshold, orientation behavior in buridan's paradigm and also phototaxis were impaired.

This puzzle prompted us to start a new investigation on $\beta$-alanine supply in the eye.

To this end, we included a second putative $\beta$-alanine source, the $\beta$-alanine synthase encoded by $p y d 3$, in our experiments.

\section{VI.P}

\section{Twelve years of clonal unit study}

\section{Kei Ito $^{1}$}

${ }^{1}$ Institute of Molecular and Cellular Biosciences, University of Tokyo

It was back in Neurofly 1996, held in Regenburg which is not so far from Würzburg, where I first presented the concept of the clonal units. A clonal unit is a set of neural circuits composed by the progeny of a single neuroblast. Interestingly, in many cases neurites of a lineage-related group of neurons form a single bundle that branches out to innervate only a few, specific areas of the fly brain. Well-investigated neuropils like the mushroom body, antennal lobe, and the central complex are all made by just a few clonal units, each of which contributes to distinct subcomponents of that neuropil. Clonal units in other brain areas also arborize in specific areas with characteristic morphology, suggesting their unique identity. Thus, clonal units are the building blocks of the fly brain architecture. It was not easy to identify the developmental mechanisms underlying the formation of such clonal units. Takeshi Awasaki, who had been in my lab, found that ectopic expression and selective silencing of cell adhesion molecules disturb the formation of the clonal fibre bundles, but the phenotype was not drastic. We suspected the role of the glial cells that insulate neighbouring clonal units. Indeed, combination of Gal4 and LexA expression induction systems enabled us to observe the malformation, albeit not total disruption, of clonal units after selective ablation of glia. These mechanisms would function redundantly to form the robust composition of neural networks. (Special thanks to Tzumin Lee for the LexA system.)

VI.2

Motorneuron complexity: re-programming the dendritic growth rate during larval development through the action of a steroid hormone.

Jan Felix Evers ${ }^{1}$, Maarten Zwart ${ }^{1}$, Owen Randlett ${ }^{1}$, Michael Bate ${ }^{1}$, Matthias Landgraf ${ }^{1}$

${ }^{1}$ Zoology, University of Cambridge

The growth of the nervous system imposes a specific constraint on the reconfiguration of existing networks: without disturbing its function, each individual network element has to change size and synaptic weight, while continuously monitoring and fulfilling its behavioural role in the network assembly. So far we understand very little about the homeostatic interplay of the partner cells involved in altering their individual size. Here we analyse the dendritic growth in a larval motor neuron (RP2) in Drosophila, which exemplifies the structural changes an identified neuron undergoes during postembryonic development. We show that RP2 roughly doubles its dendritic size at each larval stage. Starting at about $150 \mu \mathrm{m}$ in a freshly hatched $1^{\text {st }}$ instar larva, RP2 grows at an average $8 \mu \mathrm{m} /$ hour to $350 \mu \mathrm{m}$ in $2^{\text {nd }}$ instar $(24 \mathrm{~h})$, and at $27 \mu \mathrm{m} /$ hour to reach $1000 \mu \mathrm{m}$ at the beginning of the $3^{\text {rd }}$ instar $(48 \mathrm{~h})$ stage. We can demonstrate that the switch in growth speed is mediated cell autonomously by nuclear Ecdysone receptors, using isoform-specific dominant negative constructs and gain of function experiments. By contrast, neuromuscular junction growth is not affected by these manipulations. The results suggest: i) that the programme of dendritic growth is regulated through the action of steroid hormones at the level of 
the individual neuron; ii) that the complexity of neuronal input structures (dendrites) is controlled independently from that of axonal output terminals.

VI.3

Guidance through (the) Dark - Interaxonal sorting during olfactory system development

Christoph Scheper ${ }^{1}$, Sven Niehues ${ }^{1}$, Thomas Hummel ${ }^{1}$

${ }^{1}$ Institut für Neurobiologie, Universität Münster

The olfactory system of Drosophila exhibits a high degree of synaptic specificity. Olfactory receptor neurons (ORNs) expressing the same olfactory receptor form class-dependent synaptic connections with specific relay neurons (PNs), thereby forming a single glomerulus in the antennal lobe $(\mathrm{AL})$ for each class. We are interested in the developmental mechanisms that direct the axons towards their glomerular targets and mediate the synaptic specificity. ORN axons growing across the AL sort out into four projection domains according to the position of their target glomerulus. In a mosaic screen we identified multiple alleles of the complementation group verpeilt (vpt), which affect ORN axon pre-sorting, eventually leading to the innervation of a second ectopic glomerulus by the ORN47a class. Although mis-projecting vpt mutant axons maintain several class-specific abilities, they start to ectopically express the cell adhesion molecule Connectin (Con) and project into a Con-positive domain of the AL. Complementation mapping and sequencing analysis revealed that vpt affects the gene Apaf-1-related-killer (Dark), a major regulator of the DRONC caspase. We are currently testing whether the ORN targeting phenotype is caused by the loss of developmentally-regulated cell death or a non-apoptotic Caspase function during olfactory system pattering.

\section{VI.4}

\section{Diversity of Olfactory Interneurons in the Drosophila Antennal Lobe}

Maria Spletter ${ }^{l}$, Ya-Hui Chou ${ }^{1}$, Emre Yaksi $^{2}$, Rachel Wilson $^{2}$, Liqun Luo $^{3}$

${ }^{1}$ Biology, Stanford University

2 Harvard University

3 Biology, Stanford University, Howard Huges Medical Institute

In the Drosophila olfactory system, sensory information is relayed from primary olfactory receptor neuron (ORN) axons to dendrites of second-order projection neurons
(PNs) in the antennal lobe (AL). Local interneurons (LNs) in the antennal lobe play a critical role in modulating this information transfer. Relatively little is known about their development and morphology. MARCM (Mosaic Analysis with a Repressible Cellular Marker) in combination with a series of Gal4 lines that are expressed in LNs allows us to visualize and manipulate individual LNs. In contrast to previous assumptions that LNs uniformly innervate the entire AL, we find an amazing morphological and functional diversity of LN cell types. These include differences in glomerular innervation patterns, neurotransmitter expression, synaptic organization, lineage and birth timing, intrinsic electrophysiological properties, and odor response profiles. Interestingly, LN cell types are not as rigidly stereotyped in their glomerular innervation patterns as are PNs and ORNs. This wide morphological and functional diversity suggests that LNs may play previously unappreciated roles in olfactory information processing at the antennal lobe.

\section{VI.6}

Towards studies on structural plasticity in Drosophila Florian Leiss $^{1}$, Gaia Tavosanis ${ }^{1}$

1 Tavosanis Lab, Max Planck Institute of Neurobiology, Martinsried, Germany

Nervous systems can generate behavior in the light of experience. Past and present experience can affect future behavior and can elicit lasting changes in neuronal circuits. These include structural changes at the cellular level that are believed to be correlates of long-term memories in mammals. The olfactory circuit of Drosophila has proven an illuminating model to study learning and memory genetically. The second order olfactory interneurons, the mushroom body Kenyon cells, are essential for the retrieval of olfactory memories. The mushroom body calyx, containing the dendrites of these neurons as well as the axons of their presynaptic partners, the olfactory projection neurons, undergoes experience-dependent volume changes in a number of insect species including Drosophila. We have characterized the synaptic organization of the Drosophila mushroom body calyx and demonstrate that projection neuron presynaptic boutons and postsynaptic dendritic claws from Kenyon cells are organized into actin-enriched structural units called microglomeruli as previously described in other insects. We use automated image analysis to quantitatively describe microglomerular morphology and investigate if defined manipulations of the flies' sensory environment elicit structural changes in the microglomerular complexes in the mushroom body calyx. These manipulations include visual and olfactory deprivation and social isolation. First results will be presented. 
VI.9

\section{The Molecular and Cellular Basis of Bitter Taste}

Linnea Weiss ${ }^{1}$, Anupama Dahanukar ${ }^{2}$, Jae Young Kwon ${ }^{3}$, John R. Carlson ${ }^{1}$

${ }^{1}$ Mol, Cell \& Dev Biology, Yale University

${ }^{2}$ Dept of Entomology, University of California, Riverside

${ }^{3}$ Biological Science, Sungkyunkwan University

Taste stimuli are detected via neurons in chemosensory sensilla. We analyzed the electrophysiological responses of each of the $\sim 30$ labellar sensilla (L,M,S,I,P types) to structurally diverse bitter compounds and found functional differences among sensilla. Robust responses were observed in I, P and S but not L or M sensilla. S sensilla are broadly tuned but fall into two groups with distinct response profiles. I/P sensilla are more finely tuned and divide into two classes that respond to non-overlapping subsets of bitter compounds. A family of 60 gustatory receptor $(G r)$ genes encoding 68 receptors was identified. We generated flies with promoter-Gal4 drivers for 67 of the $68 \mathrm{Gr}$ proteins. At least $26 \mathrm{Gr}$-Gal4 drivers are expressed in the bitter-sensitive neurons of $\mathrm{S}, \mathrm{I}$, and/or $\mathrm{P}$ sensilla. None of the 26 putative bitter receptors is expressed in sensilla which do not respond to bitter stimuli. The broadly tuned $\mathrm{S}$ sensilla express the most drivers and fall into two classes ( $\sim 22$ or $\sim 10$ drivers), while the finely tuned I/P sensilla express 6 drivers and divide into two classes based on the complementary expression of two receptors. These classes correspond exactly to the two $\mathrm{S}$ and two I/P functional groups of sensilla. We ectopically expressed a receptor in a subset of bitter neurons and were able to confer novel bitter responses. These data strongly support a role for these 26 $\mathrm{Gr}$ proteins in bitter taste and provide a functional and receptor-to-neuron map of the fly labellum.

\section{VI.11}

Requirement of the mushroom body associated neurons for appetitive and aversive odour memories in Drosophila

Yoshinori $\mathrm{Aso}^{1}$, Ito $\mathrm{Kei}^{2}$, Martin Heisenberg ${ }^{3}$, Hiromu Tanimoto $^{1}$

${ }^{1}$ Behavioral Genetics, Max Planck Institute of Neurobiology

${ }^{2}$ Institute of Molecular and Cellular Biosciences, the University of Tokyo

${ }^{3}$ Department of Neurobiology and Genetics, University of Würzburg
Animals can form positive and negative associative memories depending on the situation when the stimulus is delivered: If it is paired with a reward or punishment, the memory will be positive or negative, respectively. To understand this differentiation at the level of neuronal circuits, we blocked activities of specific groups of neurons in Drosophila melanogaster and compared its effect on positive odour memory and that on negative one. Although the positive and negative memories drive opposite behavioural responses to the odour (i.e., approach and avoidance), the Kenyon cells of the mushroom bodies (MBs) are commonly required for both types of memories. By blocking the particular sets of Kenyon cells with $s h i^{t s I}$, we found that two hour appetitive and aversive memories requires these cells to a different extent. As a first step toward explaining this effect, we systematically characterized the expression patterns of 24 GAL4 driver lines labelling Kenyon cells. We plan to publicize the database indexing confocal images of all the GAL4 drivers analyzed. Furthermore, we systematically analyzed the anatomy and the behavioural functions of MBextrinsic neurons labeled with GAL4 drivers in positive and negative memories. Based on types of learning deficiency, these drivers are categorized into common, differential, or no requirement in these opposing memories.

VI.15

Sensory and Synaptic Specificity in the Drosophila Olfactory System

Anna Brochtrup ${ }^{1}$, Petrovic Milan ${ }^{1}$, Hummel Thomas ${ }^{1}$

${ }^{1}$ Institut für Neurobiologie, WWU Münster

In the olfactory system, sensory neurons are characterized by the expression of a single type of odorant receptor and axonal connection to a single synaptic glomerulus, thereby providing the anatomical basis for odor recognition in the brain. In mouse, the odorant receptor coordinates the receptor and synaptic target selection. In contrast, the Drosophila odorant receptors are dispensable for the development of sensory and synaptic specificity, raising the question of how these two differentiation programs are coordinated in flies.

In a screen for genes that control olfactory receptor neuron (ORN) differentiation we identified the transcription factor Sequoia as crucial for the regulation of single receptor expression and glomerular target selection. In sequoia mutants, the three ORN classes that are located in a single olfactory sensillum show a mixing of their OR expression and their axonal projections inside the glomeruli. Interestingly, the three ORNs derive from a common precursor, indicating that sequoia controls the 
class-specific differentiation of neuronal subtypes. Developmental studies revealed that sequoia is expressed in all three subtypes, which suggest that inter-cellular communication regulates sequoia activity. This is supported by subclass-specific mosaic analysis, in which cell autonomous and non-autonomous function among the three subtypes can be distinguished. This study provides the first insights into the developmental coordination of neuronal differentiation in the Drosophila olfactory system.

VI.16

A map of single octopaminergic neurons in the Drosophila brain

Sebastian Busch ${ }^{1}$, Mareike Selcho ${ }^{2}$, Kei Ito ${ }^{3}$, Hiromu Tanimoto $^{4}$

${ }^{1}$ University of Wuerzburg

${ }^{2}$ University of Fribourg

${ }^{3}$ University of Tokyo

${ }^{4}$ Max Planck Institute for Neurobiology, Martinsried

The biogenic amine octopamine modulates divers behabiors in invertebrates. It induces modulatory effects on peripheral organs, as well as regulation of more complex behaviors, such as sugar reward learning, aggression, and ethanol tolerance. However, the connectivity of individual octopaminergic neurons in the central nervous system has been largely unknown due to their anatomical complexity. We analysed the morphology of individual octopaminergic neurons in the adult Drosophila brain and subesophageal ganglion. We identified 27 distinct types of octopaminergic neurons which exhibit a clear seperation of dendritic and presynaptic regions. Each cell type targets distinct neuropils distributed throughout the brain, whereas the major dendritic ramifications are enriched in one particular brain region.

This organisation would allow them to constitute combinatorial modules potentially involved in the modulation of distinct neuronal processes. This first comprehensive characterisation of octopaminergic neurons in Drosophila provides insight into the cellular organisation of a neuromodulatory system.

\section{VI.17}

Identification of Drosophila third order olfactory neurons with a putative role in sex discrimination

Sebastian Cachero $^{1}$, Gregory Jefferis ${ }^{1}$

${ }^{1}$ Division of Neurobiology, MRC Laboratory of Molecular Biology, Cambridge, UK
Olfaction is a key sense for many animals. The olfactory system reads the chemical environment and sends information to the brain where it is used to generate behaviour. In Drosophila, the layout of the two outer layers of the olfactory system has been elucidated. Briefly, first order olfactory sensory neurons (OSNs) expressing one type of olfactory receptor project their axons to one of the $\approx 50$ glomeruli in the antennal lobe. Then second order projection neurons (PNs), whose dendrites innervate specific glomerulus, project their axons to the mushroom body and lateral horn (LH) neuropiles. We are interested in understanding how information is processed in the LH. Our first step towards that goal is to identify LH neurons.

One important use of olfaction in Drosophila is to identify suitable mating partners of the opposite sex. One male specific isoform of the gene fruitless $\left(\mathrm{fru}^{\mathrm{M}}\right)$ is believed to be expressed in neurons that are central to mating behaviour and that have been proposed to constitute a neural circuit. fru $^{\mathrm{M}}$ positive neurons have already been identified in the first two olfactory layers, OSNs and PNs.

Here we present the results of a MARCM analysis of the expression of $\mathrm{fru}^{\mathrm{M}}$ focussing on neurons of the olfactory system. We have found no fewer than 3 different neuroblast clones that generate neurons with processes in different regions of the LH. We are currently integrating this information with data from different types of PNs to find out what type of stimuli may activate these third order $\mathrm{fru}^{\mathrm{M}}+$ neurons.

VI.18

Characterisation of cholinergic interneurons in the CNS of Drosophila melanogaster

Soeren Diegelmann ${ }^{1}$, Victoria Kowalkowski ${ }^{1}$, Michael Bate $^{1}$, Matthias Landgraf ${ }^{1}$

${ }^{1}$ Department of Zoology, University of Cambridge

The locomotor circuitry of $D$. melanogaster allows the larva to perform a variety of behaviours. In contrast to sensory and motorneurons, little is known about interneurons, their specification or function. In the locomotor circuit of Drosophila acetylcholine is the major excitatory neurotransmitter. We therefore focus our research on the cholinergic interneurons and have begun to characterise them in detail. First, we identified genetic markers that serve as unique identifiers for most of the abdominal cholinergic interneurons. These markers are transcriptional regulators and results so far suggest that they operate in a combinatorial code. Second, by using the lipophilic tracer dye DiI we have identified cholinergic interneurons with projections into the dorsal 
motor neuropile as potential partners for motorneurons. Third, we fractionated the regulatory region of the Choline acetyl transferase (Cha) locus and have generated GAL4 driver lines for specific subsets. We are characterising the termination zones of these cells. To aid in the process of circuit analysis we have generated Cha driver lines for the LexA expression system. This can be used in combination with Gal4 lines to visualise and manipulate both pre- and postsynaptic partner neurons independently. These approaches enable us to study the diversity and function of cholinergic interneurons in the Drosophila ventral nerve cord and to identify among these the essential elements of the locomotor circuitry.

VI.19

\section{Expression of short neuropeptide $F$ and its receptor in the central nervous system of Drosophila}

Lina E. Enell ${ }^{1}$, Helena A.D. Johard ${ }^{1}$, Mikael A. Carlsson $^{1}$, Dick R. Nässel ${ }^{1}$

${ }^{1}$ Department of Zoology, Stockholm University, Stockholm, Sweden

Short neuropeptide F (sNPF) plays an important role in regulation of insulin-like peptides in the Drosophila brain (Lee et al. Nat Cell Biol. 10: 468, 2008), but is likely to be a multifunctional peptide. We mapped the distribution of SNPF and its receptor (SNPF-R) in the central nervous system (CNS) of Drosophila in relation to other neuronal markers. There are several hundreds of sNPF expressing neurons in the larval CNS and several thousands in the adult brain. Most of these neurons are intrinsic interneurons of the mushroom bodies, but sNPF is also expressed in numerous small interneurons of the $\mathrm{CNS}$, olfactory receptor neurons of the antennae, and in a small set of neurosecretory cells innervating the corpora cardiaca and aorta. Several of the sNPF producing neurons coexpress markers for either excitatory or inhibitory neurotransmitters, suggesting that SNPF is a co-transmitter in various CNS circuits. A few sNPF expressing neurons in the brain colocalize with the peptide corazonin and a pair of dorsal neurons in the first abdominal neuromere coexpresses SNPF and insulin-like peptide 7 . With a recently produced antiserum to the SNPF-R we labeled sets of neurosecretory cells and many other identifiable neurons, all of which match the distribution of sNPF expressing neuron terminations. Our data suggest that SNPF is a multifunctional co-transmitter throughout the Drosophila brain.
VI.20

Temperature entrainment mechanisms in Drosophila involve the novel gene nocte and peripheral organ to brain signaling

Franz Glaser ${ }^{1}$, Hanna Sehadova ${ }^{2}$, Astrid Giesecke ${ }^{2}$, Carla Gentile $^{2}$, Alekos Simoni ${ }^{2}$, Ralf Stanewsky ${ }^{2}$

${ }^{1}$ University of Regensurg

2 SBCS, Queen Mary, University of London

The endogenous clock controlling circadian rhythms is able to synchronize with environmental time cues in order to keep organisms in tune with the natural time. In addition to light, temperature cycles also work as zeitgeber for the clock, promoting both molecular and behavioural synchronization in different organisms. However, the mechanisms and genes involved in the entrainment to temperature cycles are poorly understood Two genes, nocte and norpA have been established to function in this process because the relevant mutations block temperature entrainment. nocte encodes a large glutamine rich protein, which is expressed in peripheral and central clock cells. Together with the phospholipase $\mathrm{C}$ encoded by $\operatorname{norp} A$ it may be involved in signalling temperature signals from the periphery to the clock neurons in the brain. Indeed, analysis of the molecular rhythms of different per-luc transgenic flies in the whole fly and in independent body parts under temperature entrainment conditions suggest that peripheral tissues are required to send temperature signals to the brain clock. Furthermore, reducing nocte function in substets of the adult PNS interferes with temperature entrainment of behavioural rhythms, also supporting a 'periphery to brain' signalling event. The results presented imply that the mechanisms underlying the entrainment to temperature or light cycles are fundamentally different, although ultimately both pathways need to be integrated in the central brain clock.

VI.21

\section{Translational control in the Drosophila adult brain}

Jens Hillebrand ${ }^{1}$, Scott Barbee ${ }^{2}$, Anne-Marie Cziko ${ }^{3}$, Akira Nakamura ${ }^{4}$, Roy Parker $^{3}$, Mani Ramaswami ${ }^{1}$

1 Institute for Neuroscience and Smurfit Institute for Genetics, Trinity College Dublin

2 Department of Biological Sciences, University of Denver

${ }^{3}$ Department of Molecular and Cellular Biology, University of Arizona

${ }^{4}$ RIKEN Center for Developmental Biology, Kobe 
The formation of long term memory and the underlying changes in neuronal plasticity requires new protein synthesis, which is believed to occur locally at specific synapses. This local translation is mediated through ribonucleoprotein (RNP) particles, which can contain proteins involved in mRNA translation, transport, repression and decay.

Staufen- and FMRP-containing RNPs visualized in the neurites of Drosophila neurons, also contain proteins found in P-bodies, somatic RNPs which are shown to be sites of mRNA degredation and translational control. The discovery that neuronal RNPs in Drosophila contain classic neuronal granule components, like Staufen and FMRP, as well as P-body components, indicate that those two classes of RNPs are related in composition and function. One of the shared proteins, Me31B, an evolutionary conserved P-body component found in Yeast (Dhh1), C.elegans (Cgh1) \& Mammals (RCK-P54), is required for dendritic morphogenesis and miRNA function in vivo. This brings into focus a hypothesis of potentially broad significance. Namely, that P-body components play an important role in regulation of synaptic mRNAs and thereby in synaptic plasticity. As changes in synaptic plasticity underlies memory and learning we are investigating a potential function of Me31B in the adult Drosophila brain. Progress towards a role of Me31B in local translational control in the adult brain and the dynamics of "synaptic Me31B particles" in vivo will be presented.

\section{VI.22}

Monoclonal antibodies in combination with a GAL4line reveal a distinct neuropil with high synaptic density and unknown function in the Drosophila brain.

Alois Hofbauer ${ }^{1}$, Christine Michard-Vanhée ${ }^{2}$, François Rouyer $^{2}$, Charlotte Helfrich-Förster ${ }^{1}$

${ }^{1}$ University of Regensburg, Institute of Zoology, Germany

${ }^{2}$ CNRS, Institut de Neurobiologie Alfred Fessard, Gifsur-Yvette Cedex, France

The ventrolateral protocerebrum (vlPC) is characterized by glomerular substructures among which the optic glomeruli receiving projections from the lobula are best characterized. We describe a glomerular neuropil which differs from the optic glomeruli in several aspects. This neuropil is situated dorso-anterior of the posterior optic tract (POT) and extends anteriorly close to the accessory medulla - the circadian pacemaker center of the fly.

A monoclonal antibody indicating synaptic density stains this neuropil more densely than most other areas of the brain. Two sub-compartments can be distinguished, an anterior one that seems to be organized in a peripheral "cortex" and a central core area, and a more homogeneous posterior one. Some antibodies distinguish between the compartments; others spare both in contrast to the rest of the vlPC.

A GAL4 line expressing GFP under control of the cryptochrome (cry) promoter shows in addition to the expected cry expressing neurons some additional elements in the brain. Among these a cloud of small neurons is conspicuous that seems to generate a dense fibernetwork overlapping exactly with the anterior subcompartment described above. We tried to find correlations with optic glomeruli defined by their specific innervation $(1,2)$, but none could be established so far. Possibly this area is also functionally set apart from the remaining vlPC.

1) Otsuna H, Ito K (2006) JCN 497:928-958.

2) Strausfeld NJ, Okamura JY (2007) JCN 500:166188.

VI.23

Using large-scale enhancer identification to generate new tools for neurogenetics and neuroanatomy

Arnim Jenett ${ }^{1}$, Barret D. Pfeiffer ${ }^{1}$, Teri Ngo ${ }^{1}$, Christine Murphy ${ }^{1}$, Todd R. Laverty ${ }^{1}$, Gerald M. Rubin ${ }^{1}$

${ }^{1}$ Rubin Lab, HHMI Janelia Farm Research Campus

We demonstrate the feasibility of generating thousands of transgenic Drosophila melanogaster lines in which the expression of an exogenous gene is reproducibly directed to distinct small subsets of cells in the adult brain. We expect the expression patterns produced by the collection of about 5,000 lines we are currently generating to encompass all neurons in the brain in a variety of intersecting patterns. Overlapping 3-kb DNA fragments from the flanking non-coding and intronic regions of genes thought to have patterned expression in the adult brain are inserted into a defined genomic location by site-specific recombination. These fragments are then assayed for their ability to function as transcriptional enhancers in conjunction with a synthetic core promoter designed to work with a wide variety of enhancer types. Analysis of the first couple of hundert fragments shows that a high percentage of the resulting lines drive expression patterns in the brain; the observed patterns are, on average, comprised of fewer than 100 cells or extremely broad. Our results suggest that the D. melanogaster genome contains over 50,000 enhancers and that multiple enhancers drive distinct subsets of a gene's expression in each tissue and developmental stage. We expect these lines will be valuable tools for neuroanatomy as well as for the elucidation of neuronal circuits and information flow in the fly brain. 
VI.24

\section{Sphingolipids function in neuronal connectivity}

Zheng Junfeng ${ }^{1}$, steffes Georg ${ }^{1}$, Hummel Thomas

${ }^{1}$ Institut für Neuro- und Verhaltensbiologie, Westfälische Wilhelms-Universität Münster

The convergence of olfactory axons expressing particular odorant receptor genes on spatially invariant glomeruli in the brain is one of the most dramatic examples of precise axon targeting in developmental neurobiology. In previous studies we could show that different classes of cell surface molecules expressed on projecting sensory neurons control distinct steps of olfactory system formation in drosophila. Here we report that mutations in genes controlling sphingolipids synthesis lead to similar axon connectivity defects as the loss of cell surface molecules. In a genetic mosaic screen for genes involved in olfactory neuron connectivity we identified independent mutations in the two subunits of SPT (Palmitoyltransferase), which catalyze the first step of sphingolipids biosynthesis. Sphingolipids have been shown to support cell signaling though the clustering of cell surface molecules into distinct membrane microdomains. Loss of SPT affects only a subset sensory neuron classes, which can be rescued by the neuronal expression of a wild type enzyme or by feeding sphingolipids derivatives. We determined other components of the biochemical pathway by RNAimediated gene knock down target to developing sensory neurons. Interestingly, SPT shows genetic interaction with cell adhesion molecules that are involved in sensory neuron connectivity. From these data we propose that membrane microdomains are essential for inter-axonal signaling during Drosophila nervous system development.

\section{VI.25}

Signaling substances in the central complex: distribution of neuropeptides in relation to neurotransmitters

\author{
Lily Kahsai Tesfai ${ }^{1}$, Åsa M E Winther ${ }^{2}$ \\ ${ }^{1}$ Department of Zoology, Stockholm University \\ ${ }^{2}$ Department of Zoology, Stockholm University
}

The central complex (CX) of the insect brain is believed to act as an integration center, receiving input from most parts of the brain and in Drosophila, it has been implicated in the control of both locomotor and visually correlated behaviors. The CX can be divided into several substructures, consisting of a large number of neuronal types. These neurons express classical transmitters, biogenic amines and different neuropeptides. The knowl- edge about neuropeptides and other signaling substances in the CX is limited, and in order to understand its functional role it is important to know their distribution. Therefore, we set out to map the distribution of peptides in relation to neurotransmitters. We used antisera towards neuropeptides and components of the acetylcholine, glutamate and monoamine signaling pathways in combination with Gal4 directed expression of green fluorescent protein. We employed Gal4 lines for specific interneurons and for transmitter related gene expression. The neuropeptides Drosophila tachykinin (DTK), short neuropeptide $\mathrm{F}$ and myoinhibitory peptide were mapped to three different layers of tangential F-neurons. DTK immunoreactivity was also detected in pontine neurons. Subpopulations of the DTK expressing neurons seem to coexpress acetylcholine, glutamate and possibly also octopamine/ tyramine. In general, distribution of neuropeptides in the CX seems to be widespread and diverse with regards to neuron class and colocalization with other transmitters.

VI.26

In vivo imaging of neural activities in Drosophila Johnston's organ

Azusa Kamikouchi ${ }^{1}$, André Fiala $^{2}$, Thomas Effertz ${ }^{1}$, Martin C. Göpfert ${ }^{l}$

${ }^{1}$ Sensory Systems Lab, Institute of Zoology, University of Cologne

${ }^{2}$ Department of Genetics and Neurobiology, University of Wuerzburg

Hearing in Drosophila is mediated by Johnston's organ (JO), a chordotonal stretch-receptor organ with ca. 480 primary mechanosensory neurons (JONs) that monitor stimulus-evoked antennal movements. JO has been shown to comprise 4 major JON subgroups (A,B,C,E) that target distinct regions in the brain. Whether this anatomical diversity reflects distinct functions, however, was unclear. To monitor mechanically evoked calcium signals in JON subgroups, we expressed the calcium indicator cameleon2.1 in JONs using subgroup-specific GAL4 drivers. We found that distinct JON subgroups respond to distinct stimulus types: JON subgroups $\mathrm{C}$ and $\mathrm{E}$ preferentially responded to maintained antennal deflections as imposed by static stimuli such as gravitational force. As expected for gravity-sensors, the response of these JONs was found to depend on the forcing direction, whereby their forcesensitivity matched the minute forces imposed by the earth's gravity field. Subgroups A and B, in turn, preferentially responded to antennal vibrations as imposed by dynamic stimuli such as turbulent air flows and sound. These vibration-sensitive JONs could be divided into lowfrequency and high-frequency neurons, whereby the latter 
are exquisitely sensitive and seem specialized for detecting faint sounds. The fly's JON thus houses physiologically diverse JON subgroups that seem to serve distinct mechanosensory sub-modalities and to map these submodalities onto different regions in the brain.

VI.27

In vivo optical imaging of cAMP dynamics in Drosophila brain using novel cAMP sensor proteins

Cyclic adenosine monophosphate (cAMP) is involved in numerous biological processes. However, the recording of cAMP dynamics is difficult in the living brain due to technical limitations. The spatio-temporal dynamics of cAMP signals cannot be resolved using biochemical tools. Here we report the test of a battery of DNA-encoded fluorescent sensors which allow us to real time-monitor cAMP dynamics in vivo. Those sensors feature single cAMP binding domains, derived either from human and murine Epac protein or murine $\mathrm{HCN} 2$ channel, fused to YFP- and CFP-proteins (Nikolaev et al. 2004, Nikolaev et al. 2006). We express the different cAMP sensors in the mushroom body of the fly and compare their signal-tonoise-ratios and kinetics. Our prospect is to elucidate changes in local cAMP levels in the context of learning and memory formation. First results will be presented.

\section{VI.28}

Glutamate, GABA, acetylcholine and serotonin signaling components in the lamina of the Drosophila visual system

Agata Kolodziejczyk $^{1}$, Dick Nassel ${ }^{1}$

${ }^{1}$ Department of Zoology, Stockholm University

Synaptic connections of neurons in the lamina of the optic lobe, have been comprehensively described in Drosophila, but the neurotransmitters in these circuits are poorly known. To unravel neurotransmitter circuits in the lamina we combined Gal4 driven GFP in specific lamina neurons with antisera to different components for signaling with acetylcholine, GABA, glutamate and serotonin. We suggest that acetylcholine may be used as a neurotransmitter in both L4 monopolar neurons and a novel wide-field tangential neuron (Cha-Tan). GABA is the likely transmitter of centrifugal neurons $\mathrm{C} 2$ and $\mathrm{C} 3$ and $\mathrm{GABA}_{\mathrm{B}}$ receptor immunoreactivity is seen on these neurons and the ChaTan neurons. The ionotropic $\mathrm{GABA}_{\mathrm{A}}$ receptor subunit RDL may be expressed by L4 neurons and a type of tangential neuron ( $r d l$-Tan). Vesicular glutamate transporter immunoreactivity was detected in alpha-processes of amacrine neurons and possibly in the large monopolar neurons L1 and L2. Serotonin is produced in wide field neurons with varicose processes outside the lamina synaptic neuropil. The serotonin receptors $5 \mathrm{HT} 1 \mathrm{~b}$ and 5HT2 are expressed on epithelial glia in the lamina, but not neurons, suggesting a role of serotonin in glia-mediated functions. In summary, we describe novel features of two distinct types of tangential neurons in the Drosophila lamina and assign putative neurotransmitters and some receptors to a few identified neuron types and glia.

VI.29

Poxn brain functions regulating Drosophila courtship behavior

Dimitrije Krstic $^{1}$, Werner Boll ${ }^{1}$, Markus Noll ${ }^{1}$

${ }^{1}$ Institute for Molecular Biology, University of Zurich

The Pox neuro (Poxn) gene encodes a transcription factor whose function is required in males for normal courtship behavior and fertility. One of the remarkable phenotypes of Poxn mutant males is that they do not initiate courtship towards receptive females in the dark. However, courtship in the dark can be restored by the rescue of Poxn expression in the brain. We show that at least some of these neurons are necessary for the processing of olfactory information in males as well as females. Since processing of olfactory information in general is not disturbed in flies that lack Poxn expression in the brain, we suggest that these neurons process signals elicited by olfactory pheromones and relay them to brain structures that control sexual behavior. We also show that these neurons do not express fruitless (fru), a major regulator of courtship behavior in fruit flies, which implies that fru does not control all aspects of male courtship behavior. In an attempt to understand why male courtship behavior was impaired by the missing Poxn brain function, we investigated the projection patterns of the Poxn expressing neurons in Poxn mutant and wild-type brains. We show that (i) neurons from the ventral cluster connect the antennal lobe and lateral horn, and (ii) a large number of the neurons located in the dorsolateral clusters target the ellipsoid body. In Poxn mutants, these projections are disturbed, and the development of the ellipsoid body is severely affected.

VI.30

The Drosophila Wnt5 Gene has a Role in Muscle Attachment Site Selection

Liza Lahaye ${ }^{1}$, Rene Wouda ${ }^{1}$, Anja de Jong ${ }^{1}$, L.G Fradkin $^{1}$, J.N. Noordermeer ${ }^{1}$ 
${ }^{1}$ Molecular Cell Biology, LUMC

The stereotypic pattern of the Drosophila embryonic musculature is established, in part, by inductive signaling between tendon cells and the somatic muscle fibers. Many of the genes involved in myoblast cell fate determination, fusion and muscle fiber migration and attachment have been identified. However, very little is known about the molecular mechanisms that underlie these processes. Drl, a RYK homolog, was previously shown to be required for appropriate attachment site target selection by a subset of embryonic muscles (LTMs 21-23). Drl subsequently was identified as a receptor for Wnt5-mediated commissural choice during axon guidance. Here, we present evidence that Wnt5 is also required for the attachment of the LTMs. As is observed in $d r l$ mutant embryos, wnt 5 null mutants display a 'bypass' phenotype, i.e., LTMs 21-23 extend beyond their normal tendon cells and form ectopic attachments to the body wall in $30 \%$ of the segments. Mutants homozygous for $d r l$ and lacking both copies of $w n t 5$ showed an increase to $44 \%$ of the bypass phenotype, suggesting that other Wnt receptors or Drl ligands are involved in LTM 21-23 muscle site selection during embryogenesis. Furthermore, heterozygosity for $\mathrm{drl}$ is sufficient to suppress the lethality caused by pan-muscular Wnt5 over-expression, suggesting that Wnt5 regulates muscle attachment site selection through Drl and providing further evidence that Wnt5/Drl signaling regulates both axonal pathfinding and muscle attachment site selection.

VI.31

\section{Computational Modelling of the Genetic Control of Neural Circuit Development}

Marion Langen ${ }^{1}$, Mohammed Srahna ${ }^{1}$, Yves Moreau ${ }^{2}$, Bassem A. Hassan ${ }^{1}$

${ }^{1}$ Laboratory of Neurogenetics, Department of Molecular and Developmental Genetics, VIB and KU Leuven, Leuven, Belgium

2 Bioinformatics Group, Department of Electrical Engineering, KU Leuven, Leuven, Belgium

Vision is the pre-eminent sense in both humans and Drosophila. As such, visual system neuronal connectivity has a deep impact on animal behaviour. We have previously described a Gene Interaction Network (GIN) between the Wnt, FGF and JNK signalling pathways which underlies the patterning of the connectivity diagram of a group of Drosophila visual system neurons called the DCN. However, the general rules that govern this complex process remain unclear. This is mainly due to the lack of quantitative mathematical descriptions of genetically tractable connectivity diagrams. We took advantage of a unique combination of neuro-anatomy, developmental genetics and computational modelling to provide a computational framework for the genetic control of neural circuit development. Additionaly, the computational model makes assumptions about the interactions among the DCN. These assumptions then generate genetically testable predictions used to design further genetic experiments which help fine-tuning the model in a re-iterative process. The model has thus far generated predictions about the size of an interaction neighbourhood and the genetic nature of the interactions necessary to produce the correct connectivity diagram. Genetic experiments validate these predictions and help optimizing the predictive and generalization powers of the model. In summary, we present a novel computational-genetic approach to describe the rules of neural circuit development in the Drosophila visual system.

VI.32

Genetically tracing olfactory-neural circuits in Drosophila melanogaster.

Sofia Lavista-Llanos ${ }^{1}$, Marcus C. Stensmyr ${ }^{1}$, Bill S. Hansson $^{1}$

1 Department of Evolutionary Neuroethology, Max Planck Institute for Chemical Ecology

The network of neuronal circuits accomplishing olfactory behaviour constitutes the neural substrate for processing complex odour-information. A precise manipulation of these circuits is necessary to scrutinize olfactory perception. Using the genetic model organism Drosophila, we have designed a genetic tool to express reporter proteins in circuits of synaptically connected neurons.

In order to trace olfactory-neural circuits we engineered UAS transgenic flies to target a GAL4-chimera to synaptic vesicles in olfactory neurons. This chimera is designed to release GAL4 protein at post-synaptic sites. GAL4 will next induce the expression of UAS:GAL4chimera at post-synaptic cells, turning on this transneuronal tracer in successively connected neurons.

In parallel, GAL4 will be used to induce the expression of diverse activity-dependent fluorescent proteins; which will enable detailed anatomical-depictions as well as functional imaging activity of olfactory circuits.

VI.33

Identification of Command Neurons for Wing Expansion in the Drosophila Subesophageal Ganglion

Haojiang Luan $^{1}$, Nathan Peabody ${ }^{1}$, Fengqiu Diao ${ }^{1}$, Patricia Grace ${ }^{1}$, Howard Wang ${ }^{1}$, Benjamin White ${ }^{1}$

${ }^{1} \mathrm{NIMH}$ 
Neurons that express the hormone bursicon $\left(\mathrm{N}_{\text {Burs }}\right)$ are both necessary and sufficient for wing expansion. Suppression of $\mathrm{N}_{\text {Burs }}$ blocks wing expansion, while their acute activation after eclosion induces this process. In newly eclosed adults, $\mathrm{N}_{\text {Burs }}$ typically consists of one pair of neurons in the subesophageal ganglion (the $\mathrm{B}_{\mathrm{SEG}}$ ) and seven pairs in the abdominal ganglion (the $\mathrm{B}_{\mathrm{AG}}$ ). Suppression of the $\mathrm{B}_{\mathrm{AG}}$, but not the $\mathrm{B}_{\mathrm{SEG}}$, using the c929-Gal4 driver blocks wing expansion without blocking the two motor patterns that support it: air swallowing and tonic abdominal contraction. These behaviors act in concert to raise internal pressure and force hemolymph into the wings to expand them. The $\mathrm{B}_{\mathrm{SEG}}$ thus appear to be required for behaviors underlying wing expansion, while the $\mathrm{B}_{\mathrm{AG}}$ likely mediate somatic changes required for this process. To determine whether the $\mathrm{B}_{\mathrm{SEG}}$ are sufficient to activate wing expansion behaviors, we have used our Split Gal4 system to selectively express UASTRPM8, which encodes the rat cold and menthol receptor, in all $B_{A G}$ or in only one $B_{S E G}$ neuron. Acute activation of the $\mathrm{B}_{\mathrm{AG}}$ after eclosion, using UAS-TRPM8 and a small temperature shift, fails to initiate the wing expansion motor patterns. In contrast, activation of a single $\mathrm{B}_{\mathrm{SEG}}$ neuron is sufficient to initiate these patterns and, remarkably, to drive the entire wing expansion process. These experiments therefore identify the $\mathrm{B}_{\mathrm{SEG}}$ as command neurons for wing expansion in Drosophila.

\section{VI.34}

\section{Electrophysiological characterisation of motoneurons in seizure-sensitive mutants.}

\section{Richard Marley ${ }^{1}$ \\ ${ }^{1}$ University of Manchester}

Bang sensitive flies have a significantly lowered threshold for seizure and a strong phenotypical link with human epilepsy. To date, much of this characterisation has used 'indirect' electrophysiological techniques to assess functionality of central neurons. In this study we show that larvae of bang-sensitive mutants also display lowered threshold for seizures and have used direct electrophysiological methods to determine the precise changes that occur in the CNS. A common finding in all homozygous mutants examined (Sda, Eas, Bas) is that synaptic currents recorded in aCC and RP2 motoneurons have a larger amplitude and longer duration. In heterozygotes, synaptic amplitude is still significantly larger whilst duration is not different from Canton-S wild type. As seizure-threshold is significantly reduced in heterozygotes, our observations are consistent with increased amplitude being the major determinant of seizure threshold although duration also contributes. In addition, voltage-clamp analysis of ionic currents in aCC/RP2 motoneurons shows each genotype possesses a unique elecrophysiological profile. However, the changes we observe in all mutants are not consistent with predicted changes in neurons exposed to significantly increased synaptic excitation, indicative that neuronal homeostasis in these mutations may be compromised. Taken together, our findings show significant changes to both network and neuronal properties contributing to seizure-sensitivity in these mutants.

VI.35

Generation of a neuronal map by dendritic targeting in the Drosophila embryonic motor system.

Alex Mauss ${ }^{1}$, Marco Tripodi ${ }^{1}$, Felix Evers ${ }^{1}$, Matthias Landgraf $^{1}$

${ }^{1}$ Department of Zoology, University of Cambridge

How patterns of synaptic connections are generated between neurons in the developing central nervous system (CNS) remains a central issue in neuroscience. We have studied the role of dendritic targeting in the development of the embryonic Drosophila locomotor network. We find a clear organising principle: centrally, motorneurons position their dendrites in distinct territories, which form a neural 'myotopic' map representing the distribution of their target muscles in the periphery. How is this dendritic map generated? We present evidence that the activities of the transmembrane receptors Robo and Frazzled in the dendrites regulate dendritic targeting to distinct neuropile territories with respect to the ventral midline. Our data suggest that the relative contributions of repulsive (Robo) and attractive (Frazzled) signaling determine the mediolateral positioning of motorneuron dendritic trees. Our data show that motorneuron dendrites, at least in the Drosophila ventral nerve cord, are patterned primarily by cell-intrinsic programmes that determine their responsiveness to global guidance cues such as Slit and Netrin. The same cues are also instrumental in positioning the presynaptic axons of sensory and interneurons, and may thus be central to setting up patterns of connectivity by directing the pre- and postsynaptic terminals of partner neurons to common 'meeting' regions.

VI.36

\section{A behavioral screen to map brain circuits underlying proboscis extension}

Claire McKellar ${ }^{1}$, Mei-Hsin Cheng ${ }^{1}$, Julie Simpson ${ }^{1}$

${ }^{1}$ HHMI/Janelia Farm Research Campus 
In animals that execute complex behaviors, higher planning circuits may recruit many of the same neurons and muscles for use in different behaviors. The principles underlying this potential convergence on motor outputs remain unexplored. In adult Drosophila, the simple motor action of proboscis extension is performed in different behavioral contexts, during feeding, courtship, grooming, and flight, and is influenced by motivational states such as hunger. We are carrying out behavioral screens to map both the basal motor circuit of proboscis extension and also possible planning or control circuits converging on motor outputs. Neuronal silencers or activators are targeted to different populations of neurons in large collections of GAL4 lines, to screen for neurons capable of triggering or blocking proboscis extension. Behavioral phenotypes isolated from a silencing screen to date include impaired proboscis extension during a feeding assay, excess spontaneous proboscis extension, and defects in other proboscis movements, such as labellar spreading. These lines show expression patterns in novel sets of neurons not previously known to participate in proboscis extension. Such screening techniques will reveal the neuronal components of the basal proboscis motor circuitry, and may lead to an understanding of principles underlying converging command signals from different complex behavioral circuits.

\section{VI.37}

\section{Neural Structure of the Gustatory Receptor Neurons Visualized with GAL4 Enhancer-trap System in Drosophila melanogaster}

Takaaki Miyazaki ${ }^{1}$, Kei Ito ${ }^{1}$

${ }^{1}$ Dept. of Computational Biol., Grad. Sch. of Frontier Sciences, Univ. of Tokyo; Ctr for Bioinformatics, Inst. Mol. Cell. Biosciences, Univ. of Tokyo

The main parts of the gustatory receptor neurons (GRNs) of a fly are located on the labellum of the mouth, and send their axons to the suboesophageal ganglion (SOG) via the labial nerve. Though most of the gustatory receptor genes have been identified in Drosophila melanogaster, their expression is not necessarily restricted to the GRNs, and there should be many other genes that are expressed in specific subsets of the GRNs.

To visualize subsets of the GRNs and their axons independently from the expression patterns of the gustatory receptor genes, we screened 4,000 GAL4 enhancertrap strains and identified seven lines that label GRNs but no other sensory neurons that project via the labial nerve. The axons visualized by these strains formed three major branches in the SOG, which apparently project to the mandibular, maxillary, and labellar neuromeres, respectively. We classified the terminal arborizations of these GRNs into seven areas. Among them two areas were not projected by any of the GRNs identified previously, indicating the existence of unknown types of GRNs with unique projection patterns.

We then correlated these terminal areas with the projection targets of known GRNs by double labeling. Terminal arborizations of the Gr66a-positive (bittersensing) GRNs spanned over three areas, some of which receive projections of $G r 66 a$-negative neurons. This suggests that bitter stimuli are integrated with different types of information directly in the primary gustatory center.

\section{Hindsight regulates neuronal morphogenesis in the postembryonic nervous system}

Carlos Oliva ${ }^{1}$, M.Fernanda Ceriani ${ }^{2}$, JImena Sierrralta ${ }^{3}$

${ }^{1}$ Institute for Biomedical Sciences, Universidad de Chile

${ }^{2}$ Fundacion Instituto Leloir

${ }^{3}$ Institute for Biomedical Sciences

How the neurons get its final shape is yet poorly understood. With the goal of discovery new genes that regulate the neuronal morphogenesis in the postembryonic nervous system, we are conducing a miss-expression screen in adult Drosophila neurons labeled by the atonal driver. This driver allow us to study post mitotic effects of the miss-expression because is turn it on after neuronal differentiation.

We have found that miss-expression of the transcription factor hindsight (hnt) affects the axonal guidance and dendritic arborization of this neurons. hnt encodes a nuclear zinc-finger protein that regulates dorsal closure in embryos and the cell morphology, cell fate specification, planar cell polarity and epithelial integrity during Drosophila retinal development. Studying the pattern of expression of HNT in the Drosophila brain, we discovery that, besides the retina, is expressed in the neurons of the lamina and in a few non-neuronal cells in larval and pupal central brain. The over-expression of HNT in retinal cells correlates with defects in the connectivity of the axons of the photoreceptors in the optic lobe. In addition we are studying the participation of HNT in the axon guidance of the photoreceptor cells by a loss of function approach. These results suggest that hnt is a new actor in the neuronal morphogenesis process, probably regulating JNK activation, a known target of this protein that participates in the cytoskeleton control.

Financed by ICMP08, Anillo ACT47 (JS) and CONICYT (CO). 
VI.40

Molecular and behavioural analysis of hugin and hugin-receptors in Drosophila

\author{
Marc Peters ${ }^{1}$, Michael Pankratz ${ }^{1}$ \\ 1 Development \& Genetics Unit, University of Bonn- \\ LIMES
}

Neuroanatomical studies show that the neuropeptide gene hugin is expressed in 20 neurons localized in the subesophagial ganglion. This is an area processing gustatory information and is involved in the control of feeding. A hugin promoter line driving Gal4 reveals the connectivity pattern of the hugin-expressing cells. The four major projection targets include the ring gland, the protocerebum, the pharyngeal musculature and the ventral nerve cord. Flp-out analyses show specific subsets of the hugin-expressing neurons, each projecting to a different target. To obtain more information on the function of hugin neuronal circuit in feeding control, further fly lines of different length of its regulatory element fused to Gal4 were engineered. Large fragments showed GFP-expression in all 20 neurons including the complete projection pattern of the hugin neuronal circuit. Smaller fragments led to a diminished number of GFP-expressing neurons and a lack of their specific projection targets, supporting the view that hugin-expressing neurons are arranged in clusters, and each neuron within a cluster needs a specific cis-regulatory region to express hugin. In situ hybridizations and Gal4 promoter-lines of the hugin-receptors show expression in some of the main projection targets of the hugin neuronal circuit. New fly-lines containing knockout constructs of hugin and the hugin receptors might help us to further investigate the role of the hugin neuronal circuit in feeding control.

\section{VI.41}

\section{Characterization of the Drosophila ROR tyrosine kinase receptor family}

Iveta M. Petrova ${ }^{1}$, Jean-Maurice Dura ${ }^{2}$, Jasprina N. Noordermeer ${ }^{1}$, Lee G. Fradkin ${ }^{1}$

${ }^{1}$ Leiden Univ. Med. Cen., Leiden, The Netherlands

${ }^{2}$ CNRS, Montpellier, France

ROR proteins are a conserved family of receptor tyrosine kinases. The neuronal expression of RORs in a variety of organisms and ROR mutant phenotypes suggest that they play roles in nervous system development. In mice, Ror1 and Ror2, have been found at the leading edges of actively growing neurites, indicating possible roles in axon pathfinding or synaptogenesis.
Similarly, the sole C.elegans Ror, CAM-1, contributes to the localization and/or stabilization of post-synaptic receptors and pre-synaptic sites. Wnt proteins have been recently shown to act as ligands for mammalian ROR. Wnt5a inhibits contemporaneous signaling through the canonical Wnt pathway in cultured mammalian cells. The similarity of Wnt5a and mRor2 mutant phenotypes and the overlapping expression patterns of the genes suggests that RORs likely mediate Wnt5a signaling, however, the pathway downstream of activated ROR remains largely unknown. Confirming previous reports, we find that the Drosophila ROR family members, DRor and Nrk are expressed predominantly in the nervous system. To evaluate the roles of the Drosophila ROR family in nervous system development and possible interactions with the Wnt5 protein, we are employing transgenic RNA interference and generating ROR and Nrk (neurotrophic receptor kinase) Drosophila classical mutants by imprecise P-element excision and homologous recombination.

VI.42

Temporally restricted targeting competence controls synaptic layer selection of Drosophila photoreceptor axons

Milan Petrovic $^{1}$, Thomas Hummel ${ }^{1}$

${ }^{1}$ Institut für Neurobiologie, Universität Münster

The formation of specific synaptic connections in the developing nervous system is thought to depend on the expression of distinct cell surface molecules, which allow connecting neurons to unambiguously recognize each other. The identity of these neuronal recognition molecules however is largely unknown. The visual system of Drosophila provides a well suited model to study the gene functions and developmental mechanisms underlying the establishment of synaptic specificity. The color-sensitive photoreceptors R7 and R8 project their axons sequentially into distinct layers of the medulla target area. Here, we show that the temporal expression dynamics of the $\mathrm{Zn}$ finger protein Sequoia is the major determinant of Drosophila photoreceptor connectivity into distinct synaptic layers. Neighboring R7 and R8 photoreceptors show consecutive peaks of elevated Sequoia expression, which correspond to their sequential target layer innervation. Loss of sequoia in R7 leads to a projection switch into the $\mathrm{R} 8$ recipient layer, whereas a prolonged expression in $\mathrm{R} 8$ induces a redirection of their axons into the $\mathrm{R} 7$ layer. The sequoia-induced axon targeting is mediated through the ubiquitously expressed $\mathrm{N}$-Cadherin cell adhesion molecule. We will present a model in which recognition specificity during synaptic layer formation is 
generated through a temporally restricted axonal competence to respond to broadly expressed adhesion molecules.

VI.43

\section{Embryonic development of the larval olfactory system in Drosophila.}

\author{
Laura Lucia Prieto Godino ${ }^{1}$, Michael Bate ${ }^{1}$ \\ ${ }^{1}$ Department of Zoology, University of Cambridge
}

The olfactory system of Drosophila proven to be a powerful model for studying neural network development and neural coding. Recently, the larval olfactory network, which is similar to its adult counterpart but numerically much simpler, has become a particularly attractive model for olfactory studies. However, little is known about the embryonic development of the larval olfactory system, despite its potential as a model system for studying neural network development. The aim of this work is to describe the anatomical and functional development of the larval olfactory circuit during embryogenesis. The results presented include:

- Early anatomical development of the network. By labeling neuronal sets with Acj6-Gal4, UAS-CD8GFP and anti-22c10 we were able to visualize olfactory receptor neurons $(\mathrm{ORN})$ and projection neurons $(\mathrm{PN})$ simultaneously, long before they contact each other and follow their development during embryogenesis.

- Development of chemotaxis behaviour. We have assayed embryonic and larval chemotaxis in response to several odours. Prematurely hatched embryos (normal hatching age: 21 hours after egg laying) begin to show chemotaxis behaviour in response to some odours by 20 hours AEL. Only half an hour earlier the embryos are able to crawl but fail to show any significant olfactory response.

This provides a basic though essential framework about the development of the system and sets the basis for future studies on the mechanisms underlying neural network development.

\section{VI.45}

\section{A systematic map of the Drosophila brain regions and fiber bundles}

Kazunori Shinomiya ${ }^{1}$, Kei Ito ${ }^{1}$

${ }^{1}$ Institute of Molecular and Cellular Biosciences; Department of Computational Biology, Graduate School of Frontier Sciences, The University of Tokyo
A systematic map of Drosophila brain regions is absolutely required for investigations of the brain anatomy and functions. In this study, we constructed a map of the Drosophila brain by redefining brain regions and assigned objective borders between them, intending to consolidate the structures of the fly brain, and to establish a unified terminology among insect species that will contribute comparative works on the brain. To visualize the brain structures, we mainly adopted wholemount immunostaining and the GAL4 enhancer-trap system. Six different types of antibodies were used for labeling brain structures that are applicable as landmarks of the brain regions. We also applied pan-neuronal or glial enhancer-trap strains to visualize axonal, presynaptic, postsynaptic and glial structures. Each sample was observed with the confocal microscopy in a high resolution. We also observed the brain samples with reduced silver staining to identify structures that are not visible in the other samples. By integrating the information obtained from these samples, and comparing the data with previous studies, we assigned approximately 80 subregions and 60 axon bundles within the Drosophila brain, as well as the objective boundaries of these regions by recruiting characteristic structures, such as glial sheaths, tracts and dense synaptic areas. These results are applicable as a new standard nomenclature system of the Drosophila brain regions and fiber bundles.

VI.46

Octopamine receptor 1 (OA1) is expressed by inhibitory neurons of olfactory and learning and memory centers in honey bee and Drosophila.

Irina Sinakevitch $^{1}$, Magali Iche ${ }^{1}$, Serge Birman ${ }^{1}$, Brian H Smith $^{2}$

${ }^{1}$ Genetics of Neurotransmission, IBDML-Developmental Biology Institute, CNRS-Universite de la Mediterranee, Campus de Luminy, Case 907, F-13288 Marseille Cedex 9, France.

2 Arizona State University, School of Life Science, Tempe, AZ 85287-4501

Octopamine underlies appetitive conditioning reinforcement in the honey bee and fruit fly. One octopamine receptor subtype - Apis mellifera OA1 (AmOA1), an ortholog of the fruit fly OAMB-was recently characterized. We report the distribution of AmOA1 labeling in the antennal lobes and mushroom bodies of the honey bee and fruit fly brains using antibodies raised against the AmOA1 receptor. We also studied co-localization of AmOA1 with GABA. In the antennal lobes of both species, a population of inhibitory GABAergic local 
interneurons expresses AmOA1 and addresses this receptor to their cell bodies, axons and nerve endings in the glomeruli. In the honey bee mushroom bodies, AmOA1 receptors are expressed by a subpopulation of inhibitory GABAergic feedback neurons that end in the visual and olfactory calyx neuropils and in the collar and lip zone of the vertical and medial lobes. In Drosophila, a subset of GABAergic processes in the mushroom body calyx, pedunculus and lobes express the receptor. The AmOA1 antiserum also stained Kenyon cells in the alpha', beta' and gamma lobes. In conclusion, octopamine appears to modulate inhibitory networks in both species, with specific differences occurring in the mushroom bodies. This research was funded by NIH NCRR grant RR014166 to BHS, and by a grant to IS from FRM UFP20060306548.

VI.47

Insulin-like peptide signaling in renal tubules of Drosophila is under control of stress-induced release of tachykinin-related peptide

Jeannette A. E. Söderberg ${ }^{1}$, Ryan T. Birse ${ }^{1}$, Dick R. Nässel $^{1}$

${ }^{1}$ Department of Zoology, Stockholm University, S-10691 Stockholm, Sweden

Malpighian (renal) tubules are excretory organs important for water and ion regulation, but also play roles in metabolism, immune responses and detoxification. We investigated the roles of the renal tubules, tachykininrelated peptides (DTKs) and their receptor DTKR, as well as insulin-like peptide-5 (ILP-5) and its receptor, dInR, in desiccative and nutritional stress. We show that ILP signaling locally in the renal tubules of Drosophila affects responses to stress. ILP-immunoreactivity levels in principal cells of the tubules are affected by starvation and manipulations of DTKR levels. Driving Ilp-5-and $d \operatorname{InR}$-RNAi in principal cells leads to extended survival at stress. Targeting DTKR-RNAi to the principal cells produces the same phenotype, whereas over expression of the receptor leads to abbreviated lifespan. Our findings thus suggest that metabolic stress induces hormonal release of DTKs that target the renal tubules to regulate release of ILP-5 from principal cells that acts in an autocrine loop. The increased survival at stress, seen after knock-down of DTKR, ILP-5 and dInR in tubules, may be caused by altered ILP signaling within the tubules, affecting mechanisms that regulate metabolism and lifespan.
VI.48

Investigating branch point formation in Drosophila neural lineages: Forming a circuitry roadmap

Shana Spindler ${ }^{1}$, Volker Hartenstein ${ }^{1}$

${ }^{1}$ University of California, Los Angeles

Circuit formation involves the correct localization and alignment of dendritic and axonal terminals in the brain. If branching does not occur in the proper brain compartment, required synaptic connections cannot be achieved. In Drosophila melanogaster, lineages of neurons born in the larva project a fasciculated axon bundle into the central neuropile and dedicate a position on the axon for future arbors, marked by a small tuft of filopodia. Little is known about how larval axons predetermine where branching should occur in the adult brain, and we are interested in the molecular mechanisms by which larval neurons set-up and maintain these domains on the axon. The unique organization of the Drosophila brain into lineages allows for a convenient level of visualization for both branching and circuit formation. Using lineagespecific drivers and MARCM clones, we have characterized the branch pattern for various lineages. To date, we have observed three types of branching, including delayed interstitial branching, dispersed branching, and distal branching. In addition, we have identified an important role for Par-complex proteins during branch formation in these test-lineages. In the absence of the Par-complex protein Bazooka, lineages exhibit ectopic branching in the distal portion of the axon. Establishing the mechanism for branch point formation is critical for fully understanding how neural networks are generated from larva to adult.

VI.50

Synaptic circuits in the medulla of the Drosophila visual system

Shinya Takemura ${ }^{1}$, Shuying Gao ${ }^{2}$, Chun-Yuan Ting ${ }^{2}$, Chi-Hon LuLee ${ }^{2}$, Ian Meinertzhagen ${ }^{3}$

1 Psychology and Neuroscience, Dalhousie University, Halifax, NS, Canada

${ }^{2}$ Unit on Neuronal Connectivity, NIMH, NIH, Bethesda, MD, USA

${ }^{3}$ Psychology and Biology, Dalhousie University, Halifax, NS, Canada

Tracing visual pathways from the fly's compound eye is blocked by the complexity of the second neuropile, or medulla. Extending a prior account of synaptic circuits in 
the first neuropile, or lamina, we now use medulla EM series to reconstruct input terminals to the distal medulla (lamina cells L1-L5, photoreceptors R7 and R8, medulla cell $\mathrm{T} 1$, and centrifugal cells $\mathrm{C} 2$, C3). These compare closely to forms seen from Golgi impregnation. We also identify some of their medulla cell targets. Some reconstructions were enabled by genetic reporters using the Gal4/UAS system to drive HRP expression. L1 and L2 input pathways segregate in the medulla to $\geq 5$ different target cells, which play crucial roles in motion detection. L1, reciprocally connected to L5, is presynaptic to $\geq 2$ medulla neurons; L2 is presynaptic to $\geq 3$ medulla neurons not postsynaptic to L1, two forming synaptic contacts in turn with L4. C2 and C3, alone, connect the L1 and L2 terminals and are possibly required to switch activity between these two motion-sensitive L-cell pathways. Three medulla synaptic targets of R7 and R8 probably signal colour information. $\mathrm{Tm} 5$ and $\mathrm{Tm} 9$ receive input from $\mathrm{R} 7$ and $\mathrm{R} 8$, respectively; both also receive input from L3. Multiple UV-sensitive R7 s provide input to Dm8, which is required for UV phototaxis. Using serial-EM and genetic reporters to trace circuits in the medulla, the largest neuropile of the fly's visual system, opens the way for such studies elsewhere in the fly's brain.

\section{VI.52}

\section{Synaptic Target Layer Selection in the Drosophila Visual System}

Katarina Timofeev ${ }^{1}$, Rafaela Steinborn ${ }^{1}$, Milan Petrovic ${ }^{1}$, Thomas Hummel ${ }^{1}$

${ }^{1}$ Institut fuer Neurobiologie, WWU Muenster

The establishment of neuronal networks is achieved through the precise recognition between afferent neurons and their synaptic partners in the target field. In the Drosophila visual system R7 and R8 photoreceptor cells innervate distinct layers in the medulla brain region. In previous studies we showed that the zinc finger protein Sequoia controls initial target layer selection of R7/R8 axons. Removal of sequoia in R7 leads to a switch of their axon terminals in the R8 layer, whereas seq overexpression in $\mathrm{R} 8$ results in a co-innervation in the $\mathrm{R} 7$ layer. From these data we propose that the level of seq determines cell-autonomously recognition specificity of projecting $\mathrm{R}$ cell axons.

To investigate the Sequoia-mediated axon targeting mechanism we performed a systematic RNAi-based modifier screen. In the seq overexpression background we removed distinct cell surface and intracellular signalling molecules and analysed the resulting $\mathrm{R}$ cell targeting defect. We could detect two main synaptic layer pheno- types 1) R7/8 axons stay in single synaptic layer but leave their specific topographic position and accumulate in larger axon cluster; 2) R8 axons retract from the R7 layer and terminate in a outer medulla layer. To distinguish between axon-axon and axon-target interaction we determined the R8 connectivity pattern in a sevenless background. From these results we present a model of how synaptic layer recognition specificity is molecularly determined.

\section{VI.53}

The Effects of an Altered Dopaminergic System on Behavior, Development, and Physiology in Drosophila melanogaster.

\author{
Ashby Turner ${ }^{1}$, Robin Cooper ${ }^{1}$ \\ ${ }^{1}$ Biology, University of Kentucky
}

Neuromodulators play a vital role in developing and controlling the Central Nervous System (CNS) but all of their attributes to development and behavior are not understood. Dopamine has been seen to have devastating effects if the concentrations are lowered. In humans, lower Dopamine levels are associated with Parkinson's disease and its characteristic effects on motor control. Drosophila were fed AMVT, a tyrosine hydroxalase inhibitor, at a range of concentrations. Larval development was monitored in four hour windows for the effects of reduced dopamine on rate of pupation as well as eclosion. Larvae were also examined by mouth hook and body wall movement assays. Adult flies administered AMVT were studied for stress tolerance, locomotive behavior, and coordination. AMVT appears to have little impact on rate of development in larvae, but does alter the mouth hook and body wall counts. In adults, locomotion is decreased in a dose-dependent manner. Preliminary stress tests in adults have shown a decreased tolerance to heat stress after being fed AMVT when compared to controls. Electrophysiological recordings of motor neuron activity when exposed to exogenous dopamine indicated a decreased frequency in rhythmic bursts for the larvae fed AMVT but not controls. (Funded by Arnold and Mabel Beckman Foundation)

VI.54

Trafficking of Roundabout by Commissureless in the developing central nervous system

Daan van den Brink ${ }^{1}$, Guy Tear $^{1}$

${ }^{1}$ MRC Centre for Developmental Neurobiology, King's College London 
The cells at the midline of the Drosophila central nervous system (CNS) produce a number of repellent and attractant cues that regulate the guidance of both ipsilateral and contralateral projecting neurons. One of these is Slit, which signals through the Roundabout (Robo) receptor and acts as a negative signal to steer axons away from the midline. Commissural neurons express robo but are insensitive to Slit prior to crossing the midline and become responsive to Slit after crossing. This differential sensitivity occurs since Robo protein distribution is regulated by Commissureless (Comm). Comm acts as an intracellular sorting protein that regulates the transport of Robo and is able to sequester Robo within intracellular vesicles. We have investigated the molecular mechanisms by which Comm traffics Robo. The ubiquitin ligase dNedd 4 has been shown to bind and ubiquitinate Comm and deletion of the dNedd4 binding site in Comm abolishes Comm activity. However overexpression of mutated forms of Comm reveals that ubiquitination of Comm is not necessary for Comm function. To further dissect the mechanism by which Robo is trafficked by Comm we have investigated the role of the Rab family of GTPases and additional components that regulate membrane protein traffic. The result of these studies will be presented together with preliminary work to image the distribution of Robo and Comm within the extending commissural axons in vivo.

\section{VI.55}

\section{Neuroarchitecture of aminergic system in the larval ventral ganglion}

\author{
Matthias Vömel $^{l}$, Christian Wegener ${ }^{l}$ \\ ${ }^{1}$ Emmy Noether neuropeptide group, Animal Physiology, \\ Dept. of Biology, Philipps-University
}

Biogenic amines are important signaling molecules in the CNS. Nevertheless, the projections of aminergic neurons largely remain to be characterised in detail in Drosophila. We used GAL4-driven marker gene expression and immunostainings to map presumed serotonergic, dopaminergic, and tyraminergic/octopaminergic neurons in the thoracic and abdominal neuromeres of the larval ventral ganglion relying on Fasciclin2-immunoreactive tracts as landmarks. With tyrosine hydroxylase- $(\mathrm{TH})$ or tyrosine decarboxylase 2 (TDC2)-specific GAL4-drivers, we also analyzed the distribution of ectopically expressed neuronal compartment markers in presumptive dopaminergic $\mathrm{TH}$ and tyraminergic/octopaminergic TDC2 neurons. Our results suggest that thoracic and abdominal 5-HT and $\mathrm{TH}$ neurons are exclusively interneurons, whereas most TDC2 neurons are efferent. 5-HT and TH neurons are ideally positioned to integrate sensory information and to modulate neuronal transmission within the ventral ganglion, while most TDC2 neurons appear to release amines at peripheral sites. In contrast to 5-HT neurons, TH and TDC2 neurons can be divided into morphologically different neuron subsets with separated in- and output compartments in specific neuropil regions. The threedimensional mapping of aminergic neurons is hoped to facilitate the identification of neuronal network contacts and co-localized signaling molecules, as exemplified for DOPA decarboxylase-synthesizing neurons that co-express CCAP and myoinhibiting peptides.

VI.56

\section{Dissecting Drosophila visual circuits to understand lobula plate motion processing.}

Trevor Wardill $^{1}$, Mikko Juusola ${ }^{1}$

1 Department of Biomedical Science, University of Sheffield

We wish to study how visual motion-sensitive information is routed to and processed in Drosophila lobula plate tangential neurones (LPTNs) for understanding visual behaviour. Compared to higher organisms, the relatively simple and genetically malleable connections between Drosophila LPTNs, the eyes and brain can help us to make sense of motion coding strategies. Using predominantly UV-sensitive transgenic flies, we have made preliminary recordings of intracellular voltage and $\mathrm{Ca}^{2+}$ signals in LPTNs in response to moving UV light patterns in vivo. We now wish dissect the bottom-up and top-down connections to LPTNs, and their respective roles in computing neural representations of motion signals. This we plan to do by recording changes in voltage and $\mathrm{Ca}^{2+}$ signals of LPTNs to moving UV-stimuli while switching on/off different synaptic inputs to them. This can be achieved by using the UAS-Gal4 system to express light gated channels, NpHR to inhibit or ChR2 to excite neurons, or express temperature sensitive endocytosis blocking proteins, such as shibire ${ }^{\text {ts1 }}$. The results and analysis of these experiments will be utilized for realistic mathematical modelling of motion detection.

VI.57

Neuropeptide hormone systems are evolutionarily conserved throughout the genus Drosophila

Christian Wegener ${ }^{1}$, Anton Gorbashov ${ }^{1}$

${ }^{1}$ Emmy Noether neuropeptide group, Animal Physiology, Dept. of Biology, Philipps-University 
Neuroendocrine systems in arthropods are in large part peptidergic. Not surprisingly, neuropeptide hormones are involved in the regulation of a plethora of physiological processes and behaviour. Most of these peptide hormones are produced by secretory neurons of the CNS, and are stored and released from neurohemal organs. We datamined the 12 Drosophila genomes for homologs of neuropeptide genes identified in Drosophila melanogaster, and predicted the neuropeptidome. In five species covering main phylogenetic lines of Drosophila, we then biochemically profiled the peptide hormone content of the major neurohemal organs and the epitracheal glands by MALDI-TOF mass spectrometry. Our results suggest that all Drosophila species have an identical neuropeptidome and peptide hormone complement-the same (homolog) peptide hormones are stored in the respective neurohemal organs and the epitracheal glands in all investigated species. This infers that the last common ancestor of Drosophila already had a set of neuropeptides and peptide hormones identical to that of modern fruitflies. This evolutionary stability of peptide hormone systems over more than 50 million years is remarkable, since drosophilid flies have adapted to different environments which likely require various adaptations of physiological processes that are under peptidergic regulation.

\section{VI.59}

Acquisition of electrical properties in motoneurons is specified by a combinatorial code of transcription factors

Verena Wolfram ${ }^{1}$, Edward Pym ${ }^{1}$, Tony Southall ${ }^{2}$, Andrea Brand $^{2}$, Richard Baines ${ }^{1}$

${ }^{1}$ Faculty of Life Science, Manchester University, UK

${ }^{2}$ Wellcome CRUK Gurdon Institute, Cambridge, UK

Specification of motoneurons is orchestrated by a combinatorial code of homeodomain containing transcription factors. In the Drosophila larval ventral nerve cord, expression of Even-skipped (Eve) specifies axon pathfinding and synapse formation of dorsally projecting motoneurons (aCC/RP2). By comparison, ventrally projecting motoneurons, RPs 1, 3, 4 and 5 (the RPs), are specified by the LIM-homeodomain transcription factors Islet, Lim3 and $\mathrm{dHb}$ 9. In addition, we show that the two motoneuron populations can also be distinguished by their electrophysiological properties. Our results indicate that the acquisition of these distinct electrical properties utilises a similar combinatorial code of transcription factors. Thus, overexpression of Eve is sufficient to alter electrical properties of aCC/RP2. Furthermore, we demonstrate that ectopic expression of Eve in the RPs and ectopic expression of Islet and $\mathrm{dHb} 9$ in $\mathrm{aCC} / \mathrm{RP} 2$ are similarly able to alter the electrical properties and membrane excitability of these motoneurons.

Using DamID we have identified candidate target genes to which Eve can bind. A number of these candidates could potentially alter the electrical properties of motoneurons. Here, we present verification data for several of the Eve target genes and describe their contribution to electrical properties of motoneurons.

VI.60

\section{Structure and Development of the Central Complex}

Joanna Young ${ }^{1}$, Douglas Armstrong ${ }^{1}$

${ }^{1}$ University of Edinburgh

The distinct structure of the Central Complex (CC) spanning the midline is one of the most prominent features of the Drosophila brain. The CC is highly conserved across insect species and is involved in multimodal information processing and coordination of locomotion. Although analyses have been performed on the organisation of this structure in the adult brain, few investigations have addressed its ontogeny. Characterisation of the development and neuroarchitecture of this structure is critical for elucidation of function. By employing a combination of immunohistochemical techniques, enhancer trap technology and mutant analysis, we have investigated the structure and development of the CC. We isolated 16 enhancer trap lines that showed expression in the CC. Analysis using these and three reporter lines revealed a distinct genetic subdivision of neurons and isolation of several isomorphic neuron sets in the adult brain. These lines were subsequently used to assess the development of several identified CC neurons. We determined that the CC was established by $\mathrm{P} 48$. We found that sets of neurons developed incrementally over this period starting with the HFS. From this study we have gained further insight into $\mathrm{CC}$ structure and information flow and have determined a timeline of development for several major CC neuron types. In addition, we have characterised a set of enhancer trap lines that will valuable in future developmental, structural and behavioural studies.

\section{VII.2}

\section{Selective attention gates visual information processing} in Drosophila

Shiming Tang ${ }^{1}$, Mikko Juusola ${ }^{2}$

${ }^{1}$ State Key Laboratory of Brain and Cognitive Science, Institute of Biophysics, Chinese Academy of Sciences, Beijing 
${ }^{2}$ Biomedical Science, Sheffield

Selective attention is a very important question in neuroscience and cognitive sciences, but its mechanisms are not well understood. Here we present a direct proof that Drosophila uses selective attention mechanisms in its visual processing. Our proof was obtained by recording and analysing the brain activity of a flying Drosophila for its quantifiable viewing choices.

- The key of our success was the development of a new technology that enables reliable recordings of neural activity (action potentials and field potentials, LFPs) from the lobula plates (LPs) of behaving Drosophila; previously thought almost impossible by our peers.

- At the same time, we devised a novel paradigm, which greatly amplifies the optomotor behaviour of a fly facing competing visual inputs. In our flight simulator, a flying tethered fly has to choose between two moving scenes (right and left) that compete for its interest.

- By simultaneously measuring its optomotor behaviour and neural activity in its LPs, we show that the fly brain boosts the neural output of the eye in the attended side and suppresses the output of the opposite eye. These results provide direct evidence that Drosophila uses selective attention to gate the flow of information from its eyes.

VII.4

Mushroom body miniature $B$ implicates the nuclear pore complex in mushroom body development and classical conditioning

Christine Serway ${ }^{1}$, Brian Dunkelberger ${ }^{1}$, Nicole Nolan ${ }^{1}$, Stephanie Freer ${ }^{1}$, J. Steven de Belle ${ }^{1}$

${ }^{1}$ University of Nevada Las Vegas

The brain's intricate neuronal circuitry has provided the framework for a variety of complex behaviors seen throughout the animal kingdom. Mushroom bodies (MBs) have been implicated in most insects as centers of sensory integration and association. We have shown that flies with a mutation in the gene mushroom body miniature $B(\mathrm{mbmB})$ display significant $\mathrm{MB}$ phenotypes including a reduction in calyx volume, fewer cells and axon guidance defects. Behaviorally, $m b m B$ has learning and long-term memory defects. Sterility and reduced MB volume in homozygous $m b m B$ females were indistinguishable by genetic recombination. Using sterility, we mapped $m b m B$ by complementation to 30F4-31A2. Sequencing revealed that $m b m B$ mutants have a premature stop codon in the Pendulin (Pen) gene, encoding
Drosophila Importin alpha2, a central component of the nuclear pore complex (NPC). The NPC has been implicated in axon guidance, neuronal injury response, synaptic plasticity, cell proliferation and apoptosis. We have examined PEN protein levels in adult heads. PEN is found throughout neuronal cell bodies in the brain. We are now working to rescue mutant $\mathrm{mbmB}$ behavioral and developmental phenotypes with targeted Pen transgene constructs and analyzing the cellular location of common learning and memory genes. Our detailed molecular characterization of $\mathrm{mbmB}$ provides strong evidence that trafficking across the nuclear membrane is critical for normal $\mathrm{MB}$ development, learning and long term memory.

VII.5

Does natural variation in a cGMP-dependent protein kinase (PKG) gene (foraging) underlie an adaptive response of memory to environmental heterogeneity?

Christopher J. Reaume ${ }^{1}$, Marla B. Sokolowski ${ }^{1}$, Frederic Mery $^{2}$

1 Ecology and Evolutionary Biology, University of Toronto, Toronto, Canada

${ }^{2}$ Laboratoire Evolution Génome et Spéciation, CNRS, Gif-sur-Yvette, France

Natural allelic variation in the foraging (for) gene encodes a cGMP-dependent protein kinase (PKG) that contributes to differences in learning and memory in larval and adult Drosophila melanogaster. These allelic variants are pleiotropic for differences in food-related locomotion in larvae and adults and likely have fitness consequences. We investigated if there was a relationship between learning, memory and the adaptive response to environmental heterogeneity in these natural variants. We tested their responses to environmental variation using both reversal learning and multiple stimulus learning in a Pavlovian paradigm. We predicted that the rover strain $\left(f o r^{\mathrm{R}}\right)$ would place greater emphasis on their most recent experience compared to sitter $\left(\right.$ for $\left.^{\mathrm{s}}\right)$ and sitter mutants on a rover genetic background $\left(f^{2} r^{\mathrm{s}}\right)$ since they are more likely to encounter environmental variation in nature. As expected, the $f o r^{\mathrm{R}}$ strain displayed a greater ability to reverse their response to the stimulus-shock pairing compared to $f^{s} r^{\mathrm{s}}$ and $\mathrm{for}^{\mathrm{s} 2}$. When flies were asked to learn multiple stimulus-odour pairings, the rover strain placed more emphasis on the last conditioning while decreasing the strength of the first, whereas the sitter strains placed equal emphasis on both. These data, considered alongside other known for pleiotropies, suggest that variation in for influences an adaptive response to environmental heterogeneity which may 
lead to alternative resource-use strategies in adult D. melanogaster.

VII.6

\section{Dissection of neural circuitry required for grooming behavior}

Andrew Seeds ${ }^{1}$, Julie Simpson ${ }^{1}$

1 Janelia Farm Research Campus, Howard Hughes Medical Institute

We sought to identify neural circuitry driving fruit fly grooming to gain insight into how nervous systems generate specific behaviors. Mechanosensation of dirt induces a stereotyped cleaning behavior that is amenable to both high throughput screens and detailed behavioral analysis. We used a library of enhancer-driven GAL4 lines to express the acute neural inhibitor (UAS)-shibire $e^{t s l}$ and perturb the cleaning response to dust. A screen of 1,800 lines with different brain expression patterns revealed several categories of grooming defects but did not uncover neurons whose inactivation could completely abolish the behavior. A category of lines was identified that failed to remove dust from discrete body parts and another group that lacked coordinated grooming and cleaned two body parts at a time. Anatomical analysis revealed that we isolated candidate neurons in sensory systems, interneurons, and motor outputs. We are now piecing this circuitry together by combining behavioral analysis, anatomical approaches, and calcium imaging on behaving animals to generate a model for how grooming behavior is structured in the nervous system. Consistent with our data and the work of others we postulate that grooming circuitry consists of local networks of neurons that act autonomously to drive specific components of the behavior and interneurons connecting these local modules to coordinate a cleaning response.

\section{VII.7}

\section{Cryptochrome mediates wavelength-dependent mag- netosensitivity in Drosophila melanogaster}

Taishi Yoshii ${ }^{1}$, Margaret Ahmad $^{2}$, Charlotte HelfrichFörster $^{1}$

${ }^{1}$ Department of Zoology, University of Regensburg

2 PCMP, Université Paris VI

Magnetic fields can be perceived by magnetite-based processes or radical-pair reactions involving specialized photoreceptors. The blue light photoreceptor Cryptochrome (CRY) has been proposed to represent such a photoreceptor and to mediate magnetic compass orientation in birds (Ritz et al. Biophys J 78, 2000). However, the ability of CRY to respond to magnetic fields was so far only shown in the plant Arabidopsis thaliana (Ahmad et al., Planta 225, 2007). We aimed to test the involvement of CRY in magnetoperception in the fly D. melanogaster by measuring the influence of magnetic fields on the activity rhythm. CRY is a crucial photoreceptor of the fly's circadian clock; it mediates a lightdependent slowing down of the clock finally leading to arrhythmic behaviour. We expected a stronger impact of light-activated CRY on rhythmicity in the presence of a magnetic field, if CRY can sense the magnetic field. According to the radical-pair hypothesis this response should be in addition dependent on the light colour and the field strength applied. Indeed, we found that flies exposed to dim blue light slowed down their rhythms in the presence of a magnetic field. This effect was maximal at $300 \mu \mathrm{T}$, and it disappeared completely under red-light illumination. Furthermore, $c r y^{b}$ and $c r y^{\text {out }}$ mutants did not show any response, and flies with cry overexpression in the clock neurons exhibited an enhanced response to the field. We conclude that CRY works as magnetosensor in Drosophila.

VII.8

Life without dopamine: a behavioral study of adult Drosophila unable to synthesize dopamine in the brain Thomas Riemensperger ${ }^{1}$, Hélène Coulom ${ }^{1}$, Magali Iché ${ }^{1}$, Kirsa Neuser ${ }^{2}$, Roland Strauss ${ }^{2}$, Erich Buchner ${ }^{3}$, François Rouyer ${ }^{4}$, Kazunori Kume ${ }^{5}$, Jay Hirsh ${ }^{6}$, Serge Birman ${ }^{7}$

${ }^{1}$ Genetics of Neurotransmission, IBDML-Developmental Biology Institute, CNRS-Université de la Méditerranée, Campus de Luminy, Case 907, F-13288 Marseille Cedex 9, France

2 Inst. fuer Zoologie III - Neurobiologie, Johannes Gutenberg-Universitaet, Col. -Kleinmann-Weg 2-SB II, 55099 Mainz, Germany

3 Lehrstuhl für Genetik und Neurobiologie, TheodorBoveri-Institut für Biowissenschaften, Universität Würzburg, Biozentrum, Am Hubland, 97074 Würzburg, Germany

${ }^{4}$ Institute of Neurobiology Alfred Fessard, CNRS UPR 2216, 91198 Gif-sur-Yvette Cedex, France

5 Institute of Molecular Embryology and Genetics, Kumamoto University, Kumamoto 860-0811, Japan

${ }^{6}$ Department of Biology, University of Virginia, Charlottesville, Virginia 22904, USA

${ }^{7}$ Genetics of Neurotransmission, IBDML-Developmental Biology Institute, CNRS-Université de la Méditerranée, 
Campus de Luminy, Case 907, F-13288 Marseille Cedex 9, France

The neuromodulatory role of dopamine is an inherent feature of neuron network functioning in all animals. In both vertebrates and invertebrates, dopamine plays a major role in motor control, but also in several other brain functions, like learning and memory and sexual behaviors. In Drosophila, dopamine similarly functions as a neurotransmitter/modulator, but also has a vital role in cuticle pigmentation and hardening. To learn more about the functions of dopamine in the brain, we generated Drosophila mutants lacking tyrosine hydroxylase, and thus dopamine biosynthesis, specifically in the nervous system. Surprisingly, these flies have an adult life expectancy comparable to that of wild-type flies, and normal circadian responses in bright LD or constant darkness, although dopamine is undetectable in adult brain (at a detection limit of $\sim 1-2 \%$ of controls). Compared to control flies, they have reduced activity and locomotor deficits that increase with age, and their activity is not further decreased by tyrosine hydroxylase inhibitors. Whereas their eyes function normally, as assayed by electroretinogram and optomotor response analyses, lack of neural dopamine abolishes phototaxis and strongly impairs dim light entrainment of circadian rhythms or visual tracking in the Buridan test. Therefore, dopamine in the central brain appears essential for dopaminergic control of locomotor activity and interpretation/execution of several vision-induced behavioral responses.

\section{VII.9}

\section{The logic of the neural network determining male courtship behavior in Drosophila}

\author{
Dimitrije Krstic $^{1}$, Werner Boll ${ }^{1}$, Markus Noll ${ }^{1}$ \\ ${ }^{1}$ Institute for Molecular Biology, University of Zurich
}

Courtship behavior of Drosophila melanogaster is an excellent paradigm to unravel the wiring of complex behaviors in the nervous system. In addition to determining the architecture of the neural network driving this behavior, it is crucial to elucidate how the male brain integrates the various sensory inputs during courtship. To this end, we systematically eliminated visual, olfactory, gustatory, and behavioral inputs from the courted animal and all combinations thereof, using a combination of genetics, transgenes, and ablation. By analyzing how these input changes alter the courtship behavior of a naïve male in single-choice courtship assays, we derive a model that describes the logic of a neural network regulating a complex innate behavior. The main findings are: (i) males change their courtship strategy in response to the presence or absence of light (visual tracking vs. scanning), (ii) efficient courtship depends on the complex integration of visual and chemosensory signals at daylight, and mechanosensory and chemosensory signals in the dark, (iii) the sexual orientation of males is determined by the integration of gustatory, visual, and behavioral cues from the courtee, (iv) olfactory cues are neither sufficient nor necessary to inhibit male-male courtship and represent attractive signals, and (v) chaining behavior in groups of males is observed only with taste deficient flies.

VII.10

serrano, a gene involved in $\mathrm{NaCl}$ perception in Drosophila

Georges ALVES ${ }^{1}$, Jérémy SALLE ${ }^{1}$, Stéphane DUPAS ${ }^{1}$, Sylvie CHAUDY ${ }^{1}$, Gérard MANIERE ${ }^{l}$

${ }^{1}$ UMR CNRS 5548, University of Burgundy

Gustatory information is important for animals to control behaviour when they seek for food or partners. 60 members of gustatory receptor genes have been identified in Drosophila. However little is now about mechanisms of taste perception and transduction in response to taste stimuli. In order to identify new genes involved in gustation, we performed a genetic screen using Gal4UAS system based on expression of the reporter gene in chemosensory organs and gustatory defects in larvae and adults.

We identified the serrano (sano) gene, which encodes a 778 aa protein with a leucine zipper domain, a putative transmembrane domain, and putative bipartite nuclear localization signals. sano expresssion using the PGal4 line can be detected in several neurons of the larval terminal organs. RT-PCR analysis revealed that four transcripts are expressed at different levels in the peripheral nervous system and the brain of larvae.

Loss of aversive response towards high $\mathrm{NaCl}$ concentration is observed when sano function is reduced $\left(\right.$ sano $^{\mathrm{Gal}}{ }^{4}$ homozygous or elav-Gal4-sano ${ }^{\mathrm{dsRNA}}$ ). A similar taste defect results from the blocking of neuronal transmission in sano expressing neurons (sano ${ }^{\text {Gal4 }}$-UASTNTE). However, attractive or aversive responses towards low $\mathrm{NaCl}$ concentration, sweet or bitter compounds are not affected in elav-Gal4-sano ${ }^{\mathrm{dsRNA}}$ larvae.

These results strongly suggest that Serrano function is specifically required in sensory neurons of the larval terminal organs for aversive response to high $\mathrm{NaCl}$ concentration. 
VII.11

\section{Fly-Inspired Control Algorithms in Mobile Robots-A Mutual Benefit for Basic Science and Technology}

\author{
Christian Berg ${ }^{1}$, Roland Strauss ${ }^{1}$ \\ ${ }^{1}$ Institute for Zoology III-Neurobiology, Johannes Gu- \\ tenberg-Universitaet Mainz
}

Insects choose their own goals and match their actions to the actual situation. This is accomplished by the nervous system and a wealth of different sensors and actuators. Robots can benefit greatly from the underlying principles. A Drosophila brain consists of only about $10^{5}$ neurons, still enough to provide a great flexibility in behavior. This makes the fruit fly a well suited model organism for bioinspired robots. The goal is to develop an insect brain computational model with neurogenetic and neurobiological methods. The way in which a respective robot operates can confirm the derived model or show deficits thereof. Mronz and Strauss (2008) studied orientation based on parallax motion in walking fruit flies. The results led to a computational model of object orientation implemented on a freely moving camera-equipped robot. The robot produced fly-like orientation behavior for a wide range of different object constellations without a need for object recognition. In the EU-funded SPARK II project, the architecture and the dynamical plasticity of the Drosophila brain provides the basis for an insect brain computational model in which the central complex and the mushroom bodies will play a prominent role. The design of this model is the basis for the implementation of new software and hardware for roving robots. It will provide new strategies for robotics considering higher order functions like orientation behavior, decision making, or learning.

\section{VII.12}

Double dissociation of protein-kinase $\mathrm{C}$ and adenylyl cyclase manipulations on operant and classical learning in Drosophila

Björn Brembs ${ }^{l}$

${ }^{1}$ Neurobiologie, FU Berlin

In three different discriminative conditioning experiments, the fly was fixed in space with head and thorax, but was free to beat its wings, move its legs, etc., while its yaw torque was being recorded. In order to train the fly to discriminate between a punished and an unpunished situation, an infrared light beam served as punishment.
The task was to discriminate either using classical predictors, operant predictors or a combination of both predictors. I genetically manipulated two different intracellular pathways and tested the flies in all three paradigms. The fly strains used were rut-mutants and a transgenic line expressing a specific inhibitor of PKC (PKCi) under the control of a heat-shock promoter. First, I tested the flies for learning the color predictor alone. Extending previous experiments, rut-flies are deficient in the color-learning paradigm, while flies expressing PKCi are not. The results are reversed in the purely operant paradigm. While rut-flies show unaffected learning, the learning score in flies expressing high levels of PKCi fails to reach statistical significance. Finally, the failure only of rut-flies to master the composite task is evidence that in learning situations consisting of operant and classical components, the classical predictor is learned preferentially over the operant predictor.

VII.14

Entrainment and phasing of the morning and evening oscillators in Drosophila: a new function for the PDF neuropeptide

Paola Cusumano ${ }^{1}$, André Klarsfeld ${ }^{1}$, Elisabeth Chélot $^{1}$, Marie Picot ${ }^{1}$, Benjamin Richier ${ }^{1}$, François Rouyer ${ }^{1}$

${ }^{1}$ INAF, CNRS UPR2216, Gif-sur-Yvette, France

Rest-activity rhythms in Drosophila are controlled by a clock located in the brain. This clock relies on about 150 PER-expressing neurons and perceives light through the rhodopsins of the visual system and the blue light photoreceptor cryptochrome. The bimodal activity of the flies in Light-Dark (LD) conditions is controlled by at least two oscillators: the pigment-dispersing factor (PDF) -expressing Lateral Neurons (LNs) control the morning activity, whereas the PDF-negative LNs control the evening activity. We have analyzed the entrainment of the morning and evening LN-oscillators by light and show here that they are autonomous and can use cryptochrome or the visual system to be synchronized by LD cycles. The evening oscillator is more sensitive to light, suggesting that it plays a major role in the entrainment of the brain clock. Finally, we show that $p d f^{\theta}$ mutants display an antiphasic evening oscillator in the absence of cryptochrome and a normal phase in the absence of the visual system. This indicates that the PDF neuropeptide controls the phase of the evening activity through the visual system entrainment pathway. Interestingly, this new PDF function is clock-independent and can be mediated by the large ventral lateral neurons. 
VII.15

The stress induced kinase Dp38 affects period length of the circadian clock of Drosophila melanogaster

Verena Dusik ${ }^{l}$, Charlotte Helfrich-Foerster ${ }^{l}$, Corinna Wuelbeck $^{1}$

${ }^{1}$ Institute of Zoology, University of Regensburg, Germany

The circadian oscillation of the clock proteins PERIOD, TIMELESS, CLOCK and CYCLE/BMAL is controlled by several interlocked transcriptional and translational feedback loops. It has been shown that the spatiotemporal localization, the activity and the degradation of proteins are tightly controlled by several phosphorylation steps in order to generate a $24 \mathrm{~h}$ cycle. So far only a few kinases have been identified that regulate clock protein phosphorylation such as CK1/DOUBLETIME, GSK3/SHAGGY and CK2/TIK. In addition, in vertebrates and in Neurospora two members of the mitogen activated protein kinase superfamily (MAPK), the extracellular regulated kinase (ERK) and the stress induced p38 kinase have been found to exhibit circadian activity.

To address a putative function of Drosophila p38 (Dp38) in the circadian clock of the fruit fly we analyzed the temporal and spatial expression pattern of phospho Dp38 kinase, and studied the locomotor activity as well as PERIOD phosphorylation in Dp38 mutants. We show that (1) activated Dp38 is cyclically expressed in subsets of clock neurons, (2) Dp38 mutants show a long period of the activity rhythm and (3) PERIOD phosphorylation is affected in the mutants as assessed on Western Blots. Our results indicate an involvement of Dp38 in the circadian clock.

VII.16

Courtship initiation is stimulated by mechanosensory signals in Drosophila melanogaster

Aki Ejima ${ }^{1}$, Leslie Griffith ${ }^{1}$

${ }^{1}$ Department of Biology, National Center for Behavioral Genomics and Volen Center for Complex Systems, Brandeis University

Finding a mating partner is a critical task for many organisms. It is in the interest of males to employ multiple sensory modalities to search for females. In Drosophila, vision is thought to be the most important courtship stimulating cue at long distance, while chemosensory cues are used at relatively short distance.

In this study, we show that when visual cues are not available, mechanosensation allows the male to detect the presence of a female in a large arena. When the target female was artificially immobilized, the male spent a prolonged time searching before starting courtship. This delay in courtship initiation was rescued by introduction of an active male in the arena or by playback of recorded fly movement sounds, indicating that the mechanical stimulus produced by movement stimulates courtship initiation. Mutant males expressing tetanus toxin (TNT) under the control of Gr68a-GAL4 had a defect in finding active females and a delay in courtship initiation in a large arena. Gr68a-GAL4 was found to be expressed pleiotropically not only in gustatory receptor neurons but also in mechanosensory neurons, suggesting that Gr68a-positive mechanosensory neurons provide motion detection necessary for courtship initiation. This study suggests for the first time that mechanical signals generated by a female fly have a prominent effect on males' courtship in the dark and leads the way to studying how multimodal sensory information is integrated in behavioral decision making.

VII.18

Consolidation, reconsolidation and extinction of memory processes in Drosophila melanogaster

LAGASSE Fabrice ${ }^{1}$, DEVAUD Jean-Marc ${ }^{2}$, MERY Frederic $^{1}$

${ }^{1}$ LEGS CNRS Gif sur Yvette
${ }^{2}$ CRCA University of Toulouse

After learning, memories can be stabilized over time and integrated into long-term memory (LTM) through the process of consolidation, which depends on de novo synthesis of proteins. However, Drosophila offers a striking exception since, in this model organism, LTM is not the sole stable form of memory: under specific learning conditions, anaesthesia-resistant memory (ARM) can be formed instead of LTM, through processes that are independent of protein synthesis. In this study, we take advantage of this dichotomy to address the question of the relationships between the processes leading to the stabilization of memories and those making them more fragile. Studies on other species have shown that, once stabilized, a memory can be made labile and then reinforced (reconsolidation) or inhibited (extinction) after reactivation. Here, we show that reconsolidation and extinction can be induced after olfactory conditioning in Drosophila. As in other species, the number of memory reactivations is a critical parameter that determines what process occurs. So, we asked whether different forms of reconsolidation and extinction corresponded to the ARM/ LTM dichotomy for acquisition. It does not seem to be the case: blocking protein synthesis interfered with both 
processes, regardless of the initial memory: ARM or LTM. These results thus show that Drosophila is a useful model to tackle these questions, and suggest that reconsolidation is not necessarily a mere repetition of consolidation.

VII.19

Implication of Ih potassium channel in circadian rhythms of Drosophila melanogaster

Alicia Gonzalo ${ }^{1}$, Enrique Turiégano, Isabel Molina, Laura Torroja, Inmaculada Canal

${ }^{1}$ Departamento de Biología. Universidad Autónoma de Madrid.

Organization of biological activity in daily cycles is fundamental for most organisms from bacteria to human. Complex molecular feedback loops generate rhythmic signals which control the behavioural circadian outputs. Recently, electrical activity has been shown to be important for the function of networks of clock neurons in generating rhythmic behavior. Ih is a potassium channel that generates rhythmic currents in cardiac and nervous cells. Ih is involved in many biological processes as regulation of neuronal excitability, transmission and synaptic plasticity and frequency of firing spontaneous potential actions in neurons. In order to unravel the possible contribution of Ih current in circadian locomotor behavior, we generated a null mutant for Ih. Here we present preliminary results that suggest the involvement of Ih channel in circadian rhythmicity.

VII.20

The Receptor Tyrosine Kinase Alk Signals Through Nf-1 And Is Essential For Size Determination And Learning

Jean Gouzi ${ }^{1}$, Anastasios Moressis ${ }^{1}$, Efthimios Skoulakis ${ }^{1}$

1 Institute of Molecular Biology and Genetics, BSRC Alexander Fleming

Neurofibromatosis type 1 (NF1) is among the most common human genetic disorders with symptoms including short stature and learning disabilities and results from mutations in the RasGAP neurofibromin (NF1). The fly ortholog is $\sim 60 \%$ identical to human NF1. NF1-null mutants are viable and fertile but $15 \%-20 \%$ smaller in all stages post-embryonically and associative learning deficient. We report that dNF1 and dAlk display a highly similar pattern of immunoreactivity in larval and adult
CNS, in particular in Ras2-expressing cells and $\mathrm{MB}$ neurons and both regulate MAP-kinase activation. Expression of constitutively active or activating dAlk transgenes in Ras2 larval neurons, yielded pupae 10\%$20 \%$ smaller than controls. Like NF1 mutants, these flies displayed smaller cells rather than fewer cells. More captivatingly, in a converse manner, pupae expressing transgenes that drive inactivation of dAlk signaling exhibited a $\sim 10 \%-20 \%$ larger size than controls. In a parallel approach, mutant flies displaying reduced dAlk signaling exhibited olfactory learning deficits. We report on double transgenic and double mutant studies demonstrating genetic interactions of the proteins. Collectively, these results strongly suggest that modulation of dAlk signaling accurately recapitulates NF1 growth and learning deficits in flies. Further, they highlight the importance of MAPK regulation in size determination and learning.

VII.21

Odorant-binding proteins Obp57d and Obp57e are involved in the taste perception of toxic fatty acids in oviposition-site selection

Eriko Harada ${ }^{1}$, Daisuke Haba ${ }^{1}$, Toshiro Aigaki ${ }^{1}$, Takashi Matsuo $^{1}$

${ }^{1}$ Biological sciences, Tokyo Metropolitan University

Drosophila sechellia has evolved unique behavioral adaptations to its host-plant, Morinda citorifolia, commonly known as Tahitian Noni. The ripe fruit of M.citorifolia contains hexanoic acid and octanoic acid, which are toxic to most insects. D.sechellia is resistant to these fatty acids, while other Drosophila species, including D.melanogaster, avoids them. By using interspecies hybrids between D.melanogaster and D.sechellia, we found that Odorant-binding protein57e (Obp57e) is involved in D.sechellia specific behavior. GFP reporter assay revealed that a 4-bp insertion in the D.sechellia Obp57e promoter region altered its expression in D.melanogaster background. To confirm the role of Obp57e directly, we generated a null mutant of Obp57e by gene targeting. Because Obp57e is co-expressed with Obp57d in the same cells, we also generated null mutants of Obp57d and of both Obp57d and Obp57e. The mutants showed stronger preference than wild-type flies to $\mathrm{C}_{6}-\mathrm{C}_{9}$ fatty acids. We also performed the feeding assay and the odor-based free-walking assay to explore the possibility that these OBPs are also involved in general gustatory or in olfactory perception. However, no significant difference was observed between wild-type and the mutants in the assays, indicating that these OBPs are specifically involved in oviposition-site selection. Adults' oviposi- 
tion-site determines larval food. Thus, OBP57d and OBP57e are likely to be involved in protection of larvae from toxic fatty acids.

VII.22

\section{Functional interactions of octopamine and tyramine in larval locomotion}

Julia Hill $^{1}$, Sean. T Sweeney ${ }^{1}$, John. C Sparrow ${ }^{1}$, Chris. J.H Elliott ${ }^{1}$

${ }^{1}$ Department of Biology, University of York

In invertebrates, the structural homologues of adrenaline and noradrenaline are the trace amines tyramine (TA) and octopamine (OA). In Drosophila they modulate larval crawling as shown by our analysis of two mutations in their synthesis pathway. $\mathrm{Tdc}^{\mathrm{RO} 54}$ (tyrosine decarboxylase) lacks both $\mathrm{OA}$ and $\mathrm{TA} ; \mathrm{T} \beta \mathrm{H}^{\mathrm{M} 18}$ (tyramine beta hydroxylase) lacks OA but has 10x TA level.

Both mutants show a significant reduction in speed, step distance, frequency and force of contractions during larval locomotion. $\mathrm{Tdc}^{\mathrm{RO} 54}$ mutant velocity is $60 \%$ wild type level but $\mathrm{T} \beta \mathrm{H}^{\mathrm{M} 18}$ is significantly less $(40 \%)$. In filleted preparations, application of $100 \mu \mathrm{M} \mathrm{OA}$ (but not TA) restores the contraction frequency to wild type levels. This difference between mutants suggests antagonistic action between OA and TA. However just after stressing the larvae with a hot temperature probe, velocity is the same in both mutants, only $60 \%$ of stressed wild type. As the larvae recover this antagonistic relationship between mutants is restored. The common phenotype of $\mathrm{Tdc}^{\mathrm{RO} 54}$ and $\mathrm{T} \beta \mathrm{H}^{\mathrm{M} 18}$ in other stressful assays supports this conclusion. We conclude that OA becomes more important in stressful environments and masks the role of TA. This is a novel explanation that clarifies the roles of $\mathrm{TA}$ and $\mathrm{OA}$ in normal and stressed behaviour.

\section{VII.24}

\section{Presynaptic peptidergic modulation of olfactory re- ceptor neurons}

Rickard Ignell ${ }^{1}$, Cory M Root ${ }^{2}$, Jing W Wang ${ }^{2}$, Dick R Nässel $^{3}, \stackrel{\circ}{\text { s̀ }}$ M M E Winther ${ }^{3}$

1 Department of Plant Protection Biology, Swedish University of Agricultural Sciences, Sweden

${ }^{2}$ Division of Biological Sciences, University of California, San Diego

${ }^{3}$ Department of Zoology, Stockholm University, Sweden
Presynaptic modulation of primary sensory neurons is a common strategy for gain control. Here, we have investigated the role of the neuropeptide Drosophila tachykinin (DTK) in olfactory-guided behavior and in presynaptic inhibition of olfactory receptor neurons (ORNs). We show that a subpopulation of the local interneurons (LNs) of the antennal lobe produces DTK, and that the DTK receptor DTKR is expressed in the antennal ORNs. The expression of DTKR in antennal ORNs was demonstrated by PCR and immunocytochemistry. Using two-photon microscopy we found that DTK applied to the antennal lobe suppresses presynaptic calcium in the ORNs. We used the Gal4-UAS system for targeted interference with the receptor DTKR in ORNs and monitored changes in behavior in an odor choice assay. Over expression or knock down of DTKR altered odor responses, suggesting that DTKs exert a presynaptic modulatory action. Changes in odor responses were also observed in flies with DTK levels diminished in subsets of LNs. Taken together our results suggest that Drosophila ORNs express a presynaptic neuropeptide receptor, DTKR, which serves in feedback from DTK expressing LNs of the antennal lobe.

VII.25

The neural basis of Drosophila gravity sensing and hearing

Hidehiko Inagaki ${ }^{l}$, Azusa Kamikouchi ${ }^{2}$, Thomas Effertz ${ }^{2}$, André Fiala ${ }^{3}$, Martin C. Göpfert ${ }^{2}$, Kei Ito ${ }^{4}$

${ }^{1}$ Division of Biology, Caltech

${ }^{2}$ Institute of Zoology, University of Cologne

3 Department of Genetics and Neurobiology, JuliusMaximilians-University of Würzburg

${ }^{4}$ Institute of Molecular and Cellular Biosciences, University of Tokyo

Numerous studies have shownthat visual, olfactory and gustatory sensory systems of flies share strikingfunctional and structural similarities with that of ours. Sensory mechanisms underlyingsound and gravity sensing were, however, little understood. In fly, the Johnston'sorgan (JO) in the second segment of the antenna is known as an auditory organ. Severalstudies suggested that JO is involved in gravity sensing. Anatomically, eachneuron of the JO (JON) projects to one of four areas in the brain. Here wehypothesized that gravity and sound sensing might be supported by differentsubclasses of JONs that project to different target areas. To address this, wemeasured the response characteristics of different JON subclasses, and theeffect of the selective inhibition of JON types on sound or gravity sensing. In vivo calcium imaging revealed thatdeflecting or vibrating fly's antenna 
maximally activates different subclass ofJONs. Also, behavioral assay showed that the deflection- and vibration-sensitiveneurons are necessary for gravity- and sound- sensing behavior respectively. Thus, as we speculated, the Drosophila JO houses gravity- and sound-sensitive neurons thatdiffer in their response characteristics and feed into distinct areas in thebrain. Finally, we identified higher-order neurons connected to the primarygravity- and sound-sensory neurons and found that the architecture sharessurprising similarity with that of the vestibular and auditory pathways inmammals.

VII.26

Increased fragmentation of night-time sleep in Drosophila melanogaster lacking the angiotensin-converting enzyme-related (ACER) peptidase

Elwyn Isaac ${ }^{1}$, Ahmet Carhan ${ }^{2}$, Ke Tang ${ }^{1}$, Christine Shirras $^{2}$, Alan Shirras ${ }^{2}$

${ }^{1}$ Faculty of Biological Sciences, University of Leeds

${ }^{2}$ School of Health and Medicine

The angiotensin I-converting enzyme (ACE) family of peptidases regulate levels of mammalian peptide hormones with important roles in body fluid and energy homeostasis. There are six ACE genes in Drosophila melanogaster, but only two encode functional peptidases. One of these, known as ACE-related(ACER), has only been identified in Drosophila species. In adult D. melanogaster, Acer is strongly expressed in the head, where it might be involved in regulating neuropeptide and peptide hormone signalling. In order to investigate the physiological role of Acer, we have generated two Acer mutant lines by mobilising a $\mathrm{P}$ element situated in the 5' UTR of Acer to generate precise and imprecise deletions. Adults homozygous for the imprecise deletion have no detectable ACER protein, but they develop normally and are fertile. Basal levels of Acer expression are elevated in a period null mutant, leading us to ask if ACER is involved in circadian rhythms. Although we found no evidence of a role for Acer in circadian locomotor rhythms during a $12 \mathrm{~h}$ light-dark cycle, we do find that Acer mutants have increased locomotor activity during the $12 \mathrm{~h}$ lights-off period compared to control flies, which results in sleep fragmentation and a reduction in total night-time sleep. A similar loss in the quality of sleep was observed in male adult flies fed ACER inhibitors. The loss of sleep is probably an indirect effect on locomotor activity since head Acer expression is primarily in head fatbody, a tissue with a primary role in energy homeostasis.
VII.27

\section{Dopamine and the DAMB receptor gate the transition between short-term and long-term memory in Droso- phila}

Guillaume ISABEL $L^{1}$, Kyung-An $\mathrm{HAN}^{2}$, Hélène COU$\mathrm{LOM}^{3}$, Philippe VERNIER ${ }^{4}$, Serge BIRMAN ${ }^{3}$, Thomas PREAT $^{1}$

${ }^{1}$ ESPCI-CNRS, PARIS, FRANCE

${ }^{2}$ University Park, PENNSYLVANIA, USA

${ }^{3}$ CNRS, IBDML, MARSEILLE, FRANCE

${ }^{4}$ CNRS, DEPSN, GIF SUR YVETTE, FRANCE

Dopaminergic neurons (DNs) project onto the mushroom bodies (MBs), the olfactory memory center (Friggi-Grelin et al. 2003). DNs play a positive role during acquisition (Schwaerzel et al. 2003) via the dDA1 receptor (Kim et al. 2007). To unravel the role(s) of DA in associative memory during the consolidation phase, the thermosensitive Shibire ${ }^{\text {ts }}$ protein, which allows to block reversibly specific neurons (Kitamoto, 2001), was expressed in DNs. These flies were trained with a single training session and their aversive olfactory memory tested 100-min later. When DNs are blocked during the consolidation phase, but not during learning, flies showed a strong memory increase, corresponding to a labile (cold-shock sensitive) memory. These results indicate that in normal flies DNs actively lower short-term memory (STM) levels after training.

DAMB is a DA receptor positively coupled to adenylate cyclase and mainly expressed in the $\mathrm{MBs}$ (Han et al. 1996). We showed that DAMB/+ mutants have an increased (labile) STM showing DAMB constraints labile memory in Drosophila.

To gain further insights into the role of DAMB, we tested long-term memory (LTM) capacities of the mutant after intensive conditioning. Surprisingly, LTM was abolished in DAMB and DAMB/+ flies. Similarly, we showed that LTM was abolished when dopaminergic neurons were blocked after intensive conditioning in flies. We propose that dopamine and DAMB play a key role in the dynamic transition between STM and LTM.

VII.28

CWO negatively regulates circadian transcription with bHLH-ORANGE family genes.

Taichi $Q$ Itoh $^{1}$, Kuniaki Takahashi ${ }^{2}$, Maki Ukai-Tadenuma $^{3}$, Hiroki R Ueda ${ }^{3}$, Ryu Ueda ${ }^{2}$, Teiichi Tanimura ${ }^{4}$, Akira Matsumoto 5

${ }^{1}$ Dept. Biol., Grad. School Sci., Kyushu Univ.

${ }^{2}$ Genet. Strain Res. Centr., Natl. Inst. Genet. 
${ }^{3}$ Lab. Systems Biol., Centr. Dev. Biol., RIKEN

${ }^{4}$ Dept. Biol., Fac. Sci., Kyushu Univ.

5 Centr. Res. Adv. Higher Edu., Kyushu Univ.

The core oscillation in Drosophila circadian rhythm is thought to be generated by interlocked double feedback loops of clock genes. While the framework of the molecular machinery of the feedback loops has been extensively studied, we have not yet identified all molecules involved. Our previous study using an oligonucleotide probe array identified about a thousand genes whose expression levels oscillate in fly heads. To identify novel core clock genes among them, we applied a screening system using RNA interference. We have identified a novel core clock gene, clockwork orange (cwo). The CWO protein belongs to the Stra13 subfamily which lacks the tetra-peptide domain that generally exists in the C-terminus of the other subfamilies in the bHLHORANGE family. In this study, we identified partner candidates of $c w o$ by doubly knocked down strategy. Drosophila has thirteen bHLH-ORANGE family genes, and each of them was knocked down together with cwo by using tim(UAS)-gal4 as a driver strain. Flies doubly knocked down of cwo together with its partner gene showed arrhythmicity while the single knocked down of the candidate genes revealed a little effect on the freerunning period, if any. Then we checked if there is a synergy effect between CWO and its partner candidates on suppressing the tim gene transcription by a luciferase assay in S2 culture cells. The transcriptional regulation mediated through E-box in the Drosophila clock system is more complex than previously thought.

VII.29

\section{Glia Are Critical Elements of the Circuitry Regulating Circadian Activity Rhythms}

Rob Jackson ${ }^{1}$, Fanny $\mathrm{Ng}^{1}$, Joowon $\mathrm{Suh}^{1}$

${ }^{1}$ Department of Neuroscience, Tufts University School of Medicine

Ebony is localized exclusively to glial cells and shows circadian rhythms in abundance within cells of the adult brain. Ebony mutants exhibit abnormal daily patterns of locomotor activity that can be rescued by glial cellspecific expression of the protein. Ebony encodes a synthetase which conjugates B-alanine to biogenic amines including dopamine and histamine to produce N-Balanyl-biogenic amines (NBAD for dopamine). Consistent with a close proximity of Ebony glia to dopaminergic neurons, a genetic interaction is observed between ebony and Dopamine Transporter (dDAT) mutant alleles: the extreme hyperactivity of $d D A T$ mutants is genetically suppressed by an ebony mutation, suggesting a role for NBAD in the regulation of locomotor activity. Recently, we have also shown that bimodality of daily activity is altered in the $d D A T$; ebony double mutant; the evening bout of activity is significantly diminished or eliminated. This suggests abnormal communication among the clock neuronal populations that govern the "morning" and "evening" bouts of activity. Consistent with an effect on the clock circuitry, PER clock protein abundance is increased within clock neurons and glia of the double mutant. In other studies, we have performed conditional perturbations of glial cells to define the cell populations and processes that are essential for circadian behavior. Those experiments reveal a physiological role for glia in the modulation of circadian behavior.

VII.30

\section{Modelling cognitive deficits of fragile $\mathrm{x}$ syndrome in drosophila}

Alexandros K. Kanellopoulos ${ }^{l}$, Efthimios M.C. Skoulakis $^{1}$

${ }^{1}$ Institute of Molecular Biology and Genetics, B.S.R.C “Al. Fleming", Vari-16672, Greece

A Drosophila model for Fragile X Syndrome has been developed that is based on loss-of function mutants of dfmrl, the single homolog of the FMRI gene in the Drosophila genome. Studies of these mutants uncovered neuronal and behavioural phenotypes analogous to symptoms observed in Fragile X patients. In this study, we investigated the role of dfmrl in associative and nonassociative learning in Drosophila. First, we determined whether dfmrl heterozygous mutants exhibit deficits in olfactory associative learning and memory and surprisingly, we uncovered a robust deficit. The deficit can be rescued by transgenes carrying the full length genomic fragment that harbours the entire wild type $d \mathrm{fmrl}$ gene (RES), but not by a frame-shift mutation in the coding region (FS). Furthermore, we investigated whether $d f m r l$ heterozygous mutants are deficient in habituation both as a measure of non-associative learning and to probe whether we can model attention deficit, autism and schizophrenia experienced by human FXS patients in the fly. In addition, we treated the $d f m r l$ mutants with mGluR antagonists, to restore both learning and habituation deficits. These results demonstrate the utility of the Drosophila system both as a model of behavioural deficits exhibited by FXS patients, but also as a vehicle for discovery and validation of pharmaceuticals to ameliorate them. 
VII.31

\section{Motor Skill Learning in Drosophila is a Two-Stage Process}

Bastian Kienitz ${ }^{1}$, Roland Strauss ${ }^{1}$

1 Institut fuer Zoologie III-Neurobiologie, Johannes Gutenberg-Universitaet Mainz

Drosophila melanogaster can improve a motor sequence, and learn, store and retrieve the improvements after extended practice. This is shown in a novel paradigm in which flies are walking on a rotating narrow ring with eight gaps. In an effort to compensate for the optic flow elicited by the passing-by surrounding, the flies walk on the ring preferentially against the direction of rotation and thereby cross the gaps. The training comprises five 60 -sec runs which are interrupted by 20 -min breaks. Gap crossing improves during training in terms of the success rate and the time needed. The short-term memory can open out into a long-term memory after 50 or more successful transitions. Motor skill learning is a two-stage process.

Neither improvements during training nor long-term effects were found in rutabaga ${ }^{2080}$ flies. Their defect is in the adenylyl cyclase I, a key protein of the cAMP signaling cascade mediating synaptic plasticity in Drosophila. rutabaga ${ }^{2080}$ allowed for mapping of learning and memory functions in the brain. In partial rescue experiments various GAL4 lines have been used to drive a UAS-rutabaga $^{+}$construct. The mushroom bodies (MBs) in the Drosophila brain are necessary for the short-term component of motor skill improvement. Plasticity of the alpha'-beta'-subset of MB neurons is sufficient for this component which lasts for about 40-min but is gone after one hour. Up to now, the long-term memory could be rescued by one line with a broader expression in the MBs and the central complex.

\section{VII.32}

Influence of the neuropeptide pigment-dispersing factor (PDF) on the circadian clock of short- and long-periodic rhythm mutants of Drosophila melanogaster

Christa Kistenpfennig ${ }^{1}$, Cordula Mertens ${ }^{1}$, Sinje Maruhn $^{1}$, Corinna Wülbeck ${ }^{1}$, Charlotte Helfrich-Förster ${ }^{1}$

${ }^{1}$ Institut für Zoologie, Universität Regensburg, Germany

The Drosophila neuropeptide PDF is expressed in a subset of clock neurons $\left(\mathrm{LN}_{\mathrm{V}}\right)$ of the circadian pacemaker center in the brain. PDF appears to be secreted in a circadian manner into the dorsal brain. Altered PDF levels in the dorsal brain disrupt behavioral rhythms: Flies lacking PDF exhibit weak rhythms with short periods and flies with elevated PDF levels show weak rhythms with long periods, respectively.

These results suggest that PDF interferes with the molecular oscillations in clock neurons by altering their period. It is yet unknown how PDF mediates these period changes; but PERIOD (PER) and TIMELESS (TIM), key proteins of the circadian cycle in Drosophila, are possible targets.

For this reason we studied the effect of $P d f^{\rho 1}$ on the locomotor activity of several per and tim rhythm mutants. We found a clear interaction of the per-, tim- and $P d f^{\rho 1}$ mutations on the timing of the activity phases under lightdark cycles and on the free-running periods under constant conditions (DD). To test whether PDF affects the circadian oscillation of PER and TIM we performed Western Blots on head extracts.

We observed phase advanced minima and maxima in the daily cycling of PER in different per;Pdf ${ }^{\rho 1}$ double mutants, whereas no consistent phase advances were present for PER and TIM oscillations in tim;Pdf $f^{\rho 1}$ double mutants.

VII.33

Light and temperature produce distinct entrainment patterns in the circadian clock network of the Drosophila larval brain

André Klarsfeld ${ }^{1}$, Marie Picot ${ }^{1}$, Elisabeth Chélot $^{1}$, Sébastien Malpel $^{2}$, François Rouyer ${ }^{1}$

1 Institut de Neurobiologie Alfred Fessard, CNRS UPR2216, Gif-sur-Yvette, France

${ }^{2}$ UMR 5548, CNRS and Université de Bourgogne, Dijon, France

In adult Drosophila, the brain circadian clock comprises $\sim 75$ neurons (per hemisphere). Their molecular oscillations are synchronized by light and temperature cycles. The photosensitive protein CRYPTOCHROME (CRY) and rhodopsins provide the light inputs. Temperature inputs are unknown. In the adult, light and temperature seem to phase all clock neurons similarly, but some neurons may entrain preferentially to one or to the other. Nine of the clock neurons, forming four distinct groups, are already present in larvae. We investigated how this simpler network responds to environmental inputs. At least three different light input combinations were observed among the four larval neuronal groups. Two CRY-negative dorsal neurons (the DN2s) appeared intrinsically blind. Their light entrainment, to a phase opposite that of all other clock neurons, required PIGMENT-DISPERSING FACTOR (PDF) neuropeptide signaling from four lateral neurons (LNs). In contrast, 
temperature entrainment of the DN2s was PDF-independent. The phase of their molecular oscillations did not change in thermocycles, whereas the PDF-positive LNs almost fully switched their phase, and were now in synchrony with the DN2s. As expected, this phase-switch of the LNs was reflected in the phase of adult activity after larval-only entrainment by light or temperature. Larval clock neurons thus respond very differently to light and temperature, and the CRY-negative DN2s could play a major role in temperature entrainment.

VII.34

\section{Influence of white locus on courtship behavior of} Drosophila males

Dimitrije Krstic $^{1}$, Werner Boll ${ }^{1}$, Markus Noll ${ }^{1}$

${ }^{1}$ Institute for Molecular Biology, University of Zurich

The Drosophila white gene encodes an $\mathrm{ABC}$ transporter, important for tryptophan and guanine import. Both substances are needed for the synthesis of eye pigments, but they are also precursors in the synthesis of serotonin and dopamine. Indeed, w[1118] mutants show altered levels of these neurotransmitters in the brain (Sitarman et al., 2008). As reduced levels of serotonin have been correlated with increased male sexual arousal in rabbits, rats (Frata and al., 1977), as well as cats (Ferguson et al., 1970), and ectopic expression of white has been reported to cause Drosophila males to form courtship chains (Zhang and Odenwald, 1995., Hing and Carlson, 1996), we investigated the courtship behavior of $w[1118]$ males more closely. Here we show that the lack of white function increases male sexual arousal, inducing males in a group to court each other and thus to form chains, and in single choice assays to indistinguishably court both decapitated males and females under red light. We also show that $w[1118]$ males are not only lacking visual acuity but seem to be blinded by light. In addition, we show a few examples of how the w[1118] mutation influences male behavior in single choice courtship assays.

VII.35

Sleep, diet, and longevity regulation in fruit fly

Kazuhiko Kume ${ }^{l}$, Masako Yamazaki ${ }^{1}$, Jun Tomita ${ }^{1}$, Madoka Mitsuyoshi ${ }^{1}$, Erina Sakamoto ${ }^{1}$, Taro Ueno ${ }^{1}$, Kazuhiro Takahama ${ }^{1}$, Shoen Kume ${ }^{1}$

${ }^{1}$ Inst. Molecular Embryology and Genetics, Kumamoto University
Fruit fly has recently been regarded as a new model for sleep study. Evidences were brought forward indicating the similarity between Drosophila sleep and mammalian sleep at the molecule level. We isolated a short-sleeper mutant, fumin, and proved it is defective in dopamine transporter so that their hyperactive phenotype is analogous to the effect of psychostimulants such as cocaine and amphetamine, which block this transporter function (K. Kume et al. J. Neurosci., 2005). Using this mutant, which serves as a good model of excessive dopamine signal, we are looking into the physiological relevance of the sleep. While, several studies showed sleep is essential for the survival of fruit fly, fumin retained an equivalent longevity to wild type control. The life span of fumin was almost same as control in both sexes, with different genetic backgrounds, and different temperatures, although their sleep amount was substantially reduced throughout their lives. We recently found a couple of conditions which distinguish fumin from wild type. When fed with very high caloric diet, the life span of fumin reduced dramatically, and simultaneously their sleep length decreased. This phenotype was reversed by the addition of tyrosine hydroxylase inhibitor, which reduces dopamine. The novel relationship between aging changes in sleep, diet and longevity may bring insight into the physiology of fly sleep and another interesting analogy to the mammalian sleep and aging.

VII.36

Role of homodimerization of the circadian clock protein PERIOD in Drosophila melanogaster

Johannes Lanskron ${ }^{1}$, Ko-Fan Chen ${ }^{2}$, Ralf Stanewsky ${ }^{2}$, Eva Wolf ${ }^{3}$

${ }^{1}$ Institut fur zoologie, Universitat Regensburg, Universitatsstrr., 93040 Regensburg Deutchland

${ }^{2}$ School of Biological and Chemical Science, Queen Mary University of London, Mile End Rd, London E1 4NS, United Kingdom

${ }^{3}$ Department of Structural Biology, Max-Plank Institute for Molecular Physiology, Otto-Hahn-Str. 11, 44227 Dortmund, Germany

The molecular basis of the circadian clock in Drosophila is built upon the negative feedback of period (per) and timless (tim) genes. The PER and TIM proteins heterodimerize and translocate into nucleus to repress their own transcription. PER also forms a homodimer and recently its crystal structure was resolved. We investigated the functions of the PER homodimer in vivo. According to the structural data, PER mutants predicted to break up the homodimer were generated and analyzed in transgenic flies. One mutant, M560D, resulted in a reduced ampli- 
tude of PER protein oscillations and a significant decrease of behavioural rhythmicity. Using Co-IP experiments we demonstrated PER homodimer formation in wild-type PER transgenic flies but not in M560D transgenics. Since M560D does not disrupt the interaction with TIM, disrupted homodimer formation is likely the cause of the circadian clock phenotype described above. A transcriptional reporter assay in flies showed that homodimer formation is required for full repressor activity of PER. Studying the temporal and spatial expression pattern of the homodimer-mutant PER protein in brain pacemaker neurons revealed a strong defect in nuclear localization, explaining the reduced repressor activity. Taken together, we found that the PER homodimer is important for its nuclear accumulation, feedback control of period gene transcription, and the maintenance of the circadian locomotor activity in Drosophila melanogaster.

VII.37

\section{Studying the hedonics of olfactory sensory neurons in Drosophila melanogaster}

Johannes Larsch ${ }^{1}$, Mathias Ditzen ${ }^{2}$, Leslie B. Vosshall ${ }^{2}$

1 Biology Department, University of Konstanz, 78457 Konstanz, Germany

2 Laboratory of Neurogenetics and Behaviour, The Rockefeller University, 1230 York Avenue, Box 63, New York, New York 10065, USA

In recent years, the fruit fly Drosophila melanogaster has emerged as one of the major model systems for studying olfaction. In Drosphila, olfactory sensory neurons (OSNs) express one type of olfactory receptor (OR) which defines their sensitivity to odors. Most odors activate several OSNs, thereby eliciting complex patterns of neural activity. To Drosophila, these odors can have innate hedonic values, leading to behaviorally measurable responses such as attraction or avoidance. While a large body of knowledge exists about the response profiles of different ORs, very little is known about the hedonic properties conveyed by the OSNs themselves. To study the characteristics that render a particular pattern of OSN activity as either attractive or repulsive, we have developed a new genetic tool, which allows us to induce neural activity in select OSNs of behaving adult flies. Using a modified T-Maze assay, we test whether such single input channels indeed induce stereotyped attraction and avoidance behavior. Applying this approach to OSNs located on the maxillary palps, we establish a hedonic map for this olfactory organ. In future experiments, we will extend this map to select antennal OSNs and examine the hierarchy according to which co-active OSNs contribute to behavioral avoidance and attraction.
VII.38

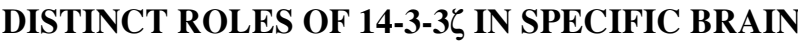 AREAS}

Flora Leptourgidou ${ }^{1}$, Efthimios Skoulakis ${ }^{1}$

1 Institute of Molecular Biology and Genetics, BSRC Alexander Fleming

The 14-3-3 protein family consists of small, soluble, acidic, dimeric proteins expressed in all eukaryotic organisms examined to date. All 14-3-3s share high sequence and functional conservation and are abundantly expressed in most areas of the central nervous system. Therefore, they have been proposed to control many of its functions. In $D$. melanogaster there are two genes encoding 14-3-3 proteins. One of them, 14-3-3 $\zeta$ (Leonardo), encodes three different isoforms and its protein products, although detected in the entire brain, accumulate preferentially in the Mushroom Bodies. Our aim is to investigate the role of $14-3-3 \zeta$ in olfactory learning and memory. Therefore, we performed a screen using different mutant and RNAi transgenic lines to assess their effect on both negatively and positively reinforced associative learning. Changes in the levels of the proteins in different neuronal subsets yields differential learning performance. 14-3-3 $\zeta$ within the MBs seems to negatively regulate learning. On the contrary, it exhibits a positive regulatory role in areas outside the MBs whereas its role in memory is still under investigation. Thus, our data indicate that as reflected by the complex distribution of $14-3-3 \zeta$ in the mammalian brain, the Drosophila homolog appears to have multiple distinct functional roles in particular CNS neurons. We discuss the possibility that this reflects differential expression of the three fly $14-3-3 \zeta$ isoforms expressed in each CNS area.

VII.39

Blockade of leucokinin release to determine possible functions of this neuropeptide in fly behaviour: Chemoreception assays

Begoña López Arias ${ }^{1}$, Jorge Martín-Joven ${ }^{1}$, Pablo Serantes $^{1}$, Pilar Herrero ${ }^{1}$

${ }^{1}$ Biology, Universidad Autónoma de Madrid

Neuropeptides of the leucokinin family, are a type of hormone that induces fluid secretion from malpighian tubules and has myotropic effects on the hindgut. These two features are shared with the peptide family of tachykinins. Previous studies have revealed leucokinin expression in the brain and ventral ganglion of Drosophila $\mathrm{CNS}$. One pair of protocerebrum neurons, located in 
the lateral horn area, surrounds the peduncles of the mushroom bodies and two pairs of suboesophageal neurons project extended processes to the tritocerebrum and through a cervical connection to the ventral ganglion. The neural functions of leucokinin remain largely unknown, particularly with respect to behaviour. Here we have studied the role of leucokinin in olfactory and gustatory perception. To this end, we have kept the LK neurons electrically silent through targeted expression of inward rectifier $\mathrm{K}^{+}$channels. In order to examine the effects of leucokinin failure, we first analyzed the dehydration response, comparing the leucokinin-silent individuals with their parents as a control. The significant differences found confirm the efficacy of the method. After, we tested the olfactory behavioural response to a set of odorants over a range of concentrations in a T-maze paradigm, in which the flies were allowed to choose between the odorant and solvent compartments. The feeding preference assays were carried out in microplates in which the flies could choose between two coloured tastes.

VII.40

\section{Mechanisms of chemotactic navigation in Drosophila larvae}

Matthieu Louis ${ }^{1}$, Thomas Huber ${ }^{2}$, Richard Benton ${ }^{3}$, Thomas Sakmar ${ }^{2}$, Leslie Vosshall ${ }^{4}$

${ }^{1}$ Systems Biology Unit, Centre for Genomic Regulation

${ }^{2}$ Laboratory of Molecular Biology and Biochemistry, The Rockefeller University

${ }^{3}$ Center for Integrative Genomics, Université de Lausanne

${ }^{4}$ Laboratory of Neurogenetics and Behavior, The Rockefeller University

Chemotaxis involves directed navigation toward attractive stimuli and away from aversive stimuli. Although this process is critical for the survival of all motile animals, the mechanisms by which higher organisms with complex nervous systems navigate through chemical gradients remain poorly described. We are studying this problem in Drosophila larvae which represent a powerful paradigm to investigate the principles of odor coding. To characterize the navigation strategies of larvae, we developed a novel chemotaxis assay where odorant conditions can be both measured and controlled. Using high-resolution computerized analysis of individual animal trajectories, we showed that Drosophila larvae advance up an odorant gradient by constantly aligning their direction of motion with that of the local odorant gradient.
We manipulated the larval olfactory system to generate animals with an altered repertoire of odorant receptors. Animals possessing only a single pair of functional olfactory sensory neurons - one on each side of the head-were generated and showed robust chemotaxis. The complexity of the system was further reduced by genetically engineering larvae with a single olfactory neuron in either the right or left side of the head. Our behavioral results provide evidence that, while bilateral olfactory input is not essential for larval chemotaxis, it enhance the accuracy of the chemotactic navigation by increasing the signal-to-noise ratio in odor detection.

VII.41

\section{Taste discrimination and learning}

Pavel Masek ${ }^{1}$, Kristin Scott ${ }^{1}$

${ }^{1}$ University of California at Berkeley

Taste recognition plays an important role in distinguishing a palatable food from a dangerous substance. In Drosophila, one class of gustatory neurons detects a large number of different sugars and mediates taste acceptance behavior, whereas a different class detects bitter compounds and mediates avoidance. Although flies can distinguish the categories of sugar versus bitter, it is not known whether they can discriminate among different sugars or among different bitter compounds. We developed a novel behavioral paradigm based on associative learning to evaluate taste discrimination. In this paradigm, flies are tested for a behavioral response (proboscis extension) to two tastants, before and after associating one tastant with a noxious heat shock. If quality discrimination occurs, flies would be expected to reduce their response specifically towards the shock-paired substance but not the unpaired one. If there is no discrimination, the reduction of their response should be unspecific. We will present data that supports the notion that flies can discriminate different sugars (or bitter compounds) based on concentration but not chemical identity.

VII.44

\section{Mechanistic insights on ras-related disorders in cognitive dysfunction}

\author{
Anastasios Moressis ${ }^{1}$, Efthimios Skoulakis ${ }^{1}$ \\ ${ }^{1}$ Institute of Molecular Biology and Genetics, BSRC \\ Alexander Fleming
}


RAS proteins play key roles in normal cell growth, malignant transformation and learning and memory. To understand the mechanisms of the cognitive deficits manifested in RAS-related disorders, we attempt genetic dissection of the RAS/RAF/ERK pathway in Drosophila olfactory learning and memory. We have previously shown that the adaptor protein DRK, a member of the ERK cascade, plays an important role in both learning and memory processes. Expanding on these studies, we show that all members of the Ras subfamily, namely $\mathrm{R} \alpha \mathrm{S} 1$, RAS2 and RAS3/RAP1, are expressed in Drosophila brain, preferentially in the Mushroom Bodies. Moreover, conditional overexpression of a constitutively active form of RAS1 in the adult MBs compromises olfactory learning and memory, whereas osmotactic responsiveness remains intact. Aiming to elucidate which effectors are responsible for the RAS1-related learning deficits observed, we overexpress in the MBs RAS1 effector loop mutants, which selectively activate distinct downstream pathways. Finally, we investigate memory performance upon selective abrogation or overexpression of each RAS protein to test whether distinct phases of memory depend on positive or negative regulation by each of the three RAS GTPases.

\section{VII.45}

Evaluating the stimuli coincidence in aversive olfactory learning of Drosophila with a semi-automated apparatus

Satoshi Murakami ${ }^{1}$, Dan Chuntao ${ }^{2}$, Brendan Zagaeski ${ }^{2}$, Yuko Maeyama ${ }^{1}$, Sam Kunes ${ }^{2}$, Tetsuya Tabata ${ }^{1}$

${ }^{1}$ Institute of Molecular and Cellular Biosciences, University of Tokyo

2 Department of Molecular and Cellular Biology, Harvard University,

In Drosophila olfactory classical conditioning, an odorant is paired with a strong unconditional stimulus such as electric shocks. It has been reported that the timing of the conditioned and unconditioned stimuli is critical for strong memory formation. For a detailed evaluation of the presentation timing, we utilized a labview-controlled semi-automated apparatus that has been devised to precisely control odor flow and the timing of electric shocks. We found that the pulsatile flow of the odor gave a higher performance index as compared with the continuous flow used in typical conditioning protocol. To monitor odor concentration during the conditioning, we employed semiconductor-based alcohol sensor. Performance index was high when the timing of electric shocks precisely coincided with the rising phase of oscillating odor concentration. On the other hand, if the timing of electric shocks coincided with the falling phase of oscillating odor concentration, performance index apparently dropped. These results reveal the temporal precision of flies' ability to detect stimuli coincidence and open up the possibility for further optimization of the olfactory conditioning protocol.

VII.46

Molecular and cellular pathways underlying a spatial orientation memory in Drosophila melanogaster

\author{
Kirsa Neuser ${ }^{1}$, Burkhard Poeck ${ }^{1}$, Roland Strauss ${ }^{1}$ \\ 1 Institute of Zoology III, Neurobiology, University \\ Mainz, Germany
}

Drosophila melanogaster flies are capable of storing the position of a hidden target after they have been lured away from their direction towards it (Neuser et al. 2008 Nature). They typically resume their approach after the detour. This spatial orientation memory is goal-driven, stable over prolonged distraction times of up to $4 \mathrm{~s}$ and the percentage of positive choices does not change during the ten consecutive trials per fly. The GABAergic ring neurons of the ellipsoid body (Hanesch et al. 1989 CTR) are necessary and their plasticity sufficient for a functional spatial orientation memory in flies. The mushroom bodies are dispensable. The protein kinase S6KII (ignorant; Putz et al. $2004 \mathrm{~J}$ Neurosci) is required to display this memory. Here, we present steps to further reveal the molecular and cellular pathway mediating the spatial orientation memory. We tested flies heterozygous for two null and homozygous lethal alleles of the $\mathrm{GABA}_{\mathrm{A}}$ receptor resistance to dieldrin ( $R d l$; Liu et al. 2007 Neuron), $R d l^{1}$ and $R d l^{f 02994}$. Furthermore, the protein kinase S6KII belongs to the ribosomal serine kinases (RSK) family, which interacts with mitogen-activated protein (MAP) kinase signalling in Drosophila. Therefore, we investigated flies mutant for the MAP kinase rolled $\left(r l^{l}\right)$. Finally, we examined, whether rescueing ignorant protein levels in subsets of central complex neurons other than that one addressed by c232-GAL4 is sufficient for restoring the memory in an ignorant mutant background as well.

VII.48

The role of octopaminergic neurons in larval Drosophila olfactory learning

Dennis Pauls ${ }^{1}$, Mareike Selcho ${ }^{1}$, Johanna Pfitzenmaier ${ }^{1}$, Desiree von Alpen ${ }^{1}$, Nanae Gendre ${ }^{1}$, Reinhard Stocker ${ }^{1}$, Andreas Thum ${ }^{1}$ 
${ }^{1}$ Department of Biology, University of Fribourg

How sensory information processing affects behavioral decisions is not well understood in any organism. Using the Drosophila larva as a simple model system, we want to dissect the neuronal substrate of associative chemosensory learning. The larval neuronal circuits mediating reward and punishment show a strong overall similarity to the circuits of an adult fly, but they are organized in a numerically much reduced and almost completely non-redundant way. In a well-established paradigm for larval olfactory learning (1) we were able to show that the dopamine receptors $\mathrm{dDA} 1$ and $D A M B$ $(2,3)$ are required for appetitive and/or aversive learning (see abstract M.Selcho). Concurrently the role of octopaminergic neurons has to be elucidated. Therefore, we used the dTdc2-GAL4 line (4) in combination with different effector lines to figure out the role of octopaminergic neurons in mediating reinforcement for olfactory associative memory formation. In an immunohistochemical approach we additionally studied the gross anatomy of the dTdc2-GAL4 line. By using the FLP-out technique (5) we were able to characterize single octopaminergic/tyraminergic neurons. Our dual behavioral and neuroanatomical approach in the larva will allow us to dissect, in a simple neuronal circuit, the neuropile regions and cells involved in olfactory learning and memory. 1: Scherer et al., 2003; 2: Kim et al., 2003, 3: Han et al., 1998, 4: Cole et al., 2005; 5: Wong et al., 2002

VII.49

The Network Governing Wing Expansion in Drosophila Is Refractory to Stimulation Before Eclosion.

Nathan Peabody ${ }^{1}$, Haojiang Luan $^{1}$, Fengqiu Diao ${ }^{1}$, Benjamin White ${ }^{I}$

${ }^{1}$ Laboratory of Molecular Biology, National Institute of Mental Health, USA

The identification and characterization of circuits underlying behavior requires the precise manipulation of activity in subsets of neurons in living, behaving animals. We have recently adapted the mammalian TRPM8 gene for use in Drosophila to permit acute neuronal stimulation in response to temperature decrements. UAS-TRPM8 reliably and reversibly activates targeted neurons with small temperature shifts and activation can be potentiated by menthol. Furthermore, TRPM8 does not require exogenous ligands and complements existing optical techniques for behavioral studies because its activation is independent of light, which is itself often a potent behavioral stimulus.

We have used the TRPM8 channel to characterize the behavioral circuit underlying wing expansion in flies. Wing expansion is a behavioral program executed directly after eclosion with both innate and adaptive components. We have used targeted TRPM8 to demonstrate that neurons that secrete the hormone bursicon are sufficient to drive wing expansion and, when activated, will do so even in environmental conditions that would normally suppress bursicon release. In contrast, activation of the bursicon-expressing neurons prior to eclosion does not initiate wing expansion, indicating that an inhibitory mechanism distinct from that used by environmental signals acts before eclosion to inhibit bursicon-expressing neurons.

VII.51

Period gene expression in four Lateral Neurons is sufficient for rhythmic activity of Drosophila melanogaster under dim light conditions

Dirk Rieger ${ }^{1}$, Corinna Wülbeck ${ }^{1}$, Charlotte HelfrichFörster $^{1}$

${ }^{1}$ Institute of Zoology, University of Regensburg

The clock-gene expressing Lateral Neurons (LN) have been shown to be crucial for Drosophila's rhythmic locomotor activity under constant conditions. Among the LN, the PDF-expressing small ventral Lateral Neurons (s$\mathrm{LN}_{\mathrm{v}}$ ) are thought to control the morning activity of the fly (=M-oscillators) and to drive rhythmic activity under constant darkness. In contrast, a fifth PDF-negative s- $\mathrm{LN}_{\mathrm{v}}$ and the dorsal Lateral Neurons $\left(\mathrm{LN}_{\mathrm{d}}\right)$ appeared to control the fly's evening activity (=E-oscillators) and to drive rhythmic activity under constant light. Here, we restricted period gene expression to four $\mathrm{LN}$ - the fifth s- $\mathrm{LN}_{\mathrm{v}}$ and three $\mathrm{LN}_{\mathrm{d}}$ - that are all thought to belong to the Eoscillators. Fascinatingly, such flies showed rather normal bimodal activity patterns under light-moonlight and constant moonlight conditions. This suggests that the four neurons are composed of $\mathrm{M}$ and $\mathrm{E}$ cells. Indeed, we found by PER-immunohistochemistry that two $\mathrm{LN}_{\mathrm{d}}$ behaved as $\mathrm{M}$ oscillators that advance their phase upon moonlight, whereas the fifth $\mathrm{s}-\mathrm{LN}_{\mathrm{v}}$ and one $\mathrm{LN}_{\mathrm{d}}$ behaved as E oscillators and delayed their activity upon moonlight. Our results suggest that $\mathrm{M}$ and $\mathrm{E}$ cells, respectively are not restricted to certain anatomically defined groups of clock neurons. Alternatively, the division in M- and Ecells may be rather flexible, so that E-cells can take the role of M-cells when the original M-cells are nonfunctional. 
VII.52

\section{Environmental Cues Driving Exploration in Droso- phila melanogaster: Attending the Edge}

Gregg Roman ${ }^{1}$

${ }^{1}$ Dept. Biology and Biochemistry, University of Houston

Drosophila melanogaster, when placed into a circular open field arena, initially displays a high level of activity, followed by a stable, lower level of spontaneous activity. Active exploration is the foremost component of the elevated initial activity phase. Visually impaired and blind mutants, such as $w^{1118}$ and norpA $A^{7}$, are defective in attenuating the active exploration phase, presumably because of an inability to habituate the novelty. We have sought to better understand the proximate and ultimate causes for exploration in flies by dissecting the role of environmental factors in shaping this behavior. Both wild type and visual impaired flies spend a majority of their time at the edge of an open field arena, and also show a significant corner preference in square arenas. The preference for edges and corners could be due to thigmotaxis. However, flies do not attend to extra surfaces placed internally within the arena. Interestingly, by darkening the edge of a circular arena, we were able to rescue the $w^{1118}$ defect in the attenuation of exploration. The behavior of wild type Canton-S and the blind norpA ${ }^{7}$ were not affected by the darkened edge. It is probable that increasing the contrast of the arena's edge allowed the $w^{1118}$ flies to visually habituate to novelty. These data strongly suggest that flies are visually attending to the arena's edge during exploration and are displaying neither centrophobicity nor thigmotaxis in an open field arena.

VII.53

\section{Visual selective attention in Drosophila}

Preeti Sareen $^{1}$, Reinhard Wolf ${ }^{1}$, Martin Heisenberg ${ }^{1}$

${ }^{1}$ Lehrstuhl für Genetik und Neurobiologie, Biozentrum, Universität Würzburg

At any point in time, the volume of sensory information reaching the visual system is enormous. This makes it critical to filter out the irrelevant. One way to accomplish this is to confine certain processing steps to only a part of the visual field and to shift the "window of attention" to where this processing is needed. This operating mode is called visual selective attention (VSA). As for Drosophila, VSA has been demonstrated during tethered flight at a torque meter ${ }^{1}$. Flies can restrict some visual processing to only parts of the visual field (window of attention) and can shift that window deliberately. It has also been shown that VSA in flies can be guided through presentation of additional visual stimuli ${ }^{2,3}$ or by application of additional odour stimuli ${ }^{3}$. Flies have been shown to be attending to the side at which the guiding stimulus was presented. In recent studies we found, that a vertical bar with flickering lights (white LEDs) causes the flies to direct their VSA to the opposite direction, if the stimulus is presented in a fronto-lateral position, suggesting that this guiding stimulus is aversive to the flies. VSA of flies therefore seems able to be guided by certain cues and the nature of these cues could be attractive or aversive.

VII.55

\section{Behavioural assay for appetitive visual memory}

Christopher Schnaitmann ${ }^{1}$, Tilman Triphan ${ }^{2}$, Simon Triphan $^{2}$, Martin Heisenberg ${ }^{2}$, Hiromu Tanimoto ${ }^{1}$

${ }^{1}$ Max-Planck-Institute of Neurobiology, Am Klopferspitz 18, 82152 Martinsried, Germany

${ }^{2}$ Department of Neurobiology and Genetics, University of Würzburg, Am Hubland, 97074 Würzburg, Germany

Visual associative memory of adult Drosophila has been intensely investigated using the behavioural assay " flight simulator" where a tethered fly learns to avoid a particular visual stimulus by receiving a heat beam. Despite its success, the mechanism for appetitive visual memory is largely unknown. Thus, we decided to develop a conditioning assay for appetitive visual associative learning in the adult fly. Starved flies are trained en masse to associate one of two different visual stimuli (e.g. blue and green light) with sugar reward. In the test phase, both rewarded and control visual stimuli were presented in the absence of the sugar reward, and the flies' preference was measured as associative memory. The trained flies showed a conditioned approach: The preference for a rewarded cue was significantly higher than that for the same cue of the reciprocally trained group, where the other cue had been conversely rewarded. Several parameters including the number of training sessions, order of reinforcement, inter-stimulus interval, context and starvation were examined to optimize the memory. As this assay is versatile, easy to set up, and yields consistent conditioned behaviour, it has a potential to contribute to the investigation of the neuronal 
differences underlying aversive and appetitive visual learning.

VII.56

Free-flight kinematics in Drosophila performing voluntary take-off maneuvers

Peter Schützner ${ }^{1}$, Fritz-Olaf Lehmann ${ }^{1}$

${ }^{1}$ Neurobiology, University of Ulm

Sensorimotor coordination during flight results from a feedback cascade that converts sensory information into complex motor commands. Since tethered flight conditions partially interrupt this cascade, we reconstructed wing-, body- and head motion in freely maneuvering fruit flies using three-dimensional high-speed video $(3.5 \mathrm{KHz}$ frame rate). The obtained data show flight in a natural context under authentic sensory feedback and include the dynamics and aerodynamics of body and wings. To distinguish between fixed-action motor patterns and conventional feedback control, we analyzed the repertoire of free flight maneuvers in Drosophila including voluntary take-off behavior. Compared to tethered flight, freeflight kinematics shows a remarkable variance on a stroke-by-stroke basis. We found distinct asynchronies in wing rotation at the stroke reversals and differences in stroke angle between both wings within the first 9 stroke cycles after flight initiation. Clap-and-fling force enhancement is rare and occurs in only $54 \%$ of the maneuvers. Similar to larger blow flies, the animals yielded compensatory head motions of up to $20^{\circ}$ angular rotation while stabilizing their gaze towards the random-dot visual environment. Although the overall take-off behavior appears to be similar among the tested flies, the elevated variance in kinematic patterns suggests that take-off behavior in fruit flies is shaped by both feedback control and fixed-action motor patterns.

\section{VII.57}

\section{Dopaminergic neurons in the Drosophila larva}

Mareike Selcho ${ }^{1}$, Dennis Pauls ${ }^{1}$, Johanna Pfitzenmaier ${ }^{1}$, Kyung-An $\mathrm{Han}^{2}$, Nanae Gendre ${ }^{1}$, Reinhard Stocker ${ }^{1}$, Andreas Thum ${ }^{1}$

${ }^{1}$ University of Fribourg, Switzerland

${ }^{2}$ Pennsylvania State University, USA

How sensory information processing affects behavioral decisions is not well understood in any organism. Using the Drosophila larva as a simple model system, we want to dissect the neuronal substrate of associative learning. The larval neuronal circuits for chemosensory learning show a strong overall similarity to the adult circuits, but they are organized in a numerically much reduced and almost completely non-redundant way. This allows a facilitated accessibility on the genetic, molecular, cellular and physiological level. Unlike previous studies in adult Drosophila and in honeybees $(1,2,3,4)$, the role of biogenic amines in the processing of reward and punishment in Drosophila larvae remains to be elucidated. In an immunohistochemical approach we studied the gross anatomy of the dopaminergic neurons by using $\mathrm{TH}$ GAL4 (5), as well as the expression patterns of the dopamine receptors dDA1 and DAMB $(6,7)$. Using the FLP-out technique (8) we then investigated the anatomy of single DA neurons in the brain. Thereby untangling locally distinct innervation onto different neuropiles, including the mushroom bodies, a brain structure involved in olfactory learning. Our approach may allow us to dissect in a simple neuronal circuit, the neurons and neuropile regions involved in mediating reward and punishment for olfactory learning and memory. (1) Schwaerzel et al., 2003; (2) Kim et al., 2007; (3) Hammer et al., 1993; (4) Vergoz et al., 2007; (5) Friggi-Grelin et al., 2003; (6) Kim et al., 2003; (7) Han et al., 1998; (8) Wong et al., 2002

VII.58

Operant Behavior in Walking Drosophila

Nidhi Singhal ${ }^{1}$, André Fiala ${ }^{1}$

${ }^{1}$ Genetics and Neurobiology, University of Wuerzburg

In operant behavior animals can achieve a reward or avoid a punishment through their own action. We are interested in the neuronal mechanisms mediating punishing or rewarding events in the fly's brain. To this end we have established a novel paradigm in which a heat punishment is linked with directed walking behaviour. Flies are placed onto a styrofoam ball which is suspended by an air stream. The flies are attached to a manipulator by their thorax but can walk on a styrofoam ball which is free to rotate on an air cushion. Thus the flies are fixed in space but can freely choose to walk in any desired direction. The rotations of the styrofoam ball are monitored electronically. We train the animals by linking a heat punishment with a particular direction of walking. Thereby, the animal can associate this direction with the punishment and ultimately avoids walking in the punished direction. Using this approach we hope to identify neurons involved in mediating of punishment in the Drosophila brain. First results will be presented. 
VII.59

\section{Visual pattern memory in Drosophila is different for "novelty learning" and heat conditioning}

Narendra Solanki ${ }^{1}$, Reinhard Wolf ${ }^{2}$, Martin Heisenberg ${ }^{2}$

${ }^{1}$ Institute of Genetics \& Neurobiology, University of Würzburg

${ }^{2}$ Institute of Genetics \& Neurobiology

"Novelty Choice" is a visual learning paradigm without any obvious reinforcer, instead it relies on the fly's spontaneous preference for suddenly appearing new patterns compared to the existing old patterns ${ }^{1}$. Whereas in the so-called standard learning experiment in the flight simulator the acquisition of visual pattern memory consists of two components ${ }^{2}$. First is classical learning of pattern parameters associated with heat punishment, and second is operant component in which the fly learns to use its behavior to avoid the punishment. In the novelty paradigm the retrieval of pattern memory is exclusively operant. This is consistent with the finding, that rutabaga mutants (rut) have no learning phenotype in the novelty paradigm. It is known that associative learning and memory requires an intact rut protein, a type- 1 adenylyl cyclase considered to be a coincidence detector between US and $\mathrm{CS}^{3}$. So far five visual parameters as Height, Size, Vertical Compactness, Edge Orientation and Color $^{4}$ can be trained in the standard experiment. Surprisingly it appears that not all of them can be "learned" in the novelty paradigm. In the present study we show that of the tested two parameters, novelty choice can be shown only with height and not with edge orientation. Besides the rutabaga gene, novelty learning seems not to require the mushroom bodies. The ellipsoid body however, seems to be necessary for this task. We also found that ignorant mutants are defective in novelty learning.

\section{VII.61}

\section{Second-Order Conditioning in Drosophila melanoga- ster}

Christopher J. Tabone ${ }^{1}$, J. Steven de Belle ${ }^{1}$

${ }^{1}$ University of Nevada, Las Vegas

The study of complex, higher-order learning behavior has been a focus of neurobiology and behavioral research for decades. Training Drosophila melanogaster utilizing classic differential conditioning protocols provides the means by which researchers can elucidate the mechanisms underlying the behavior of learning and memory. Here we present an analysis of second-order conditioning using a modified version of the Pavlovian olfactory paradigm. We demonstrate that flies display an ability to form second-order memories in which a previously conditioned stimulus (CS1) can serve as an unconditioned stimulus (US) for a novel conditioned stimulus (CS2) in a second episode of training. These findings agree with similar results previously reported by other researchers utilizing the Drosophila flight simulator. We also demonstrate that flies are capable of distinguishing single odors from complex mixtures during this second-order training paradigm. Furthermore, we investigate the idea of "reconsolidation" versus extinction during CS1 memory retrieval and and discuss its possible role as an internal unconditioned stimulus during the formation of secondorder memories. The study of complex behavior, such as second-order conditioning in Drosophila melanogaster, may help to uncover specific neuronal circuits responsible for the storage and retrieval of conditioned stimuli during differential conditioning.

VII.63

\section{Searching for Sleep-Related and Arousal-Related Genes in Drosophila}

Jun Tomita ${ }^{l}$, Madoka Mitsuyoshi ${ }^{1}$, Erina Sakamoto ${ }^{1}$, Masako Yamazaki ${ }^{1}$, Nobuaki Shiraki ${ }^{1}$, Shoen Kume ${ }^{1}$, Kazuhiko Kume ${ }^{1}$

1 Institute of Molecular Embryology and Genetics, Kumamoto University

The regulation mechanisms of sleep and arousal are poorly understood. A fruit fly, Drosophila melanogaster has emerged as a useful system to facilitate a molecular understanding of sleep and arousal. Rest behaviors of Drosophila share features with mammalian sleep in that their amount is regulated by both circadian rhythm and homeostatic mechanism, and that they are associated with decreased responsiveness to sensory stimuli. We have discovered hyperactive sleepless mutant flies, fumin (fmn) and demonstrated that the behavioral phenotype is caused by a genetic lesion in the dopamine transporter (DAT) gene. Importantly DAT is also involved in sleep and arousal regulations in mammals. In order to identify molecular candidates involved in sleep and arousal, we used DNA microarray to compare gene expression in heads of control and fmn flies collected every $4 \mathrm{~h}$ for $24 \mathrm{~h}$ under $12 \mathrm{~h}$ light/dark conditions. There were 136 genes with significant difference in expression levels throughout a day between control and fmn flies $(\mathrm{P}<0.05)$. Fifty nine of these 136 genes were up-regulated and the remaining 77 genes including DAT gene were down-regulated in fmn flies. Quantitative RT-PCR analyses were performed to validate the microarray results. Using the transposon insertion or RNAi lines of the candidate genes, their 
possible involvement in the regulation of locomotor activities and sleep were examined with Drosophila activity monitoring system.

VII.64

\section{The Modular Structure of Climbing Control in Drosophila}

Tilman Triphan ${ }^{1}$, Roland Strauss ${ }^{1}$

${ }^{1}$ Institut für Zoologie III-Neurobiologie, Johannes Gutenberg-Universität Mainz

Drosophila melanogaster is extraordinarily maneuverable, even when forced to walk. By a sophisticated climbing behavior flies can overcome gaps in their walkway which are up to $1.6 x$ wider than their body length (Pick and Strauss, 2005; Curr. Biol.). The behavior is used in an adaptive manner, i.e. if the gap gets insurmountably broad, a climbing attempt will not be elicited. Once climbing, a fly will try to reach the opposite side with its front legs, thereby forming a bridge. To increase the reach, the fly will move its body into the gap whereby the hind legs grasp the proximal edge of the gap. It will then lift up the body with the middle legs.

We analyze this complex motor behavior with the help of two orthogonal high-speed video-cameras. We found mutant lines that fail at specific details of this behavior, hinting at the modular structure of climbing control. In addition to lines which hardly initiate climbing even at easily manageable gaps, we now report on the line sisyphus, which engages in climbing even at clearly insurmountable gap widths. Other lines have been identified which fail to lift up the body with the middle legs or fail to position their body over the gap before engaging in climbing. Several mutant lines take a correct decision to climb based on the visual estimation of gap width. However, on approaching the gap they are losing direction to the opposite side. The common feature of the latter lines are structural defects in the protocerebral bridge.

VII.65

Three state model for the evaluation of arousal and sleep in fruit fly

Taro Ueno ${ }^{1}$, Jun Tomita ${ }^{1}$, Masako Yamazaki $^{1}$, Shoen Kume $^{1}$, Kazuhiko Kume ${ }^{1}$

${ }^{1}$ University of Kumamoto
Sleep and wakefulness are two alternate states and have been demonstrated in many animals including fruit fly. Sleep in Drosophila is defined based on behavioral study. Using DAM (Drosophila activity monitoring) system, the number of crossing infrared beam which lie in the center of a tube is recorded and the inter-count-interval is calculated. It has been shown that prolonged resting states in Drosophila show common properties with mammalian sleep, like increased threshold to outside stimuli. However, in mammals, the state of immobility contains awake and alert state. We showed that the resting state in Drosophila also could be subdivided into two states (sleep or resting alert) based on the response to mechanical stimulations. Using video monitoring system, we also found that flies show a small movement which correspond to the state available to react to the stimuli (resting alert). Based on these classifications, we conducted further study including pharmacological analysis of sleep. Flies administrated with caffeine only slightly increased the total locomotor activities and slightly decreased the amount of rest. However, it dramatically lowered the arousal threshold and increased small movements, such as glooming. We propose that three state model better describes the details of sleep or awake state.

VII.66

Functional characterization of medulla neurons using a behavioral assay for color vision

Nina Vogt ${ }^{1}$, Claude Desplan ${ }^{1}$

${ }^{1}$ Biology, New York University

In the Drosophila compound eye, color information is detected by the inner photoreceptors R7 and R8. Their projections bypass the lamina part of the optic lobe and directly innervate the medulla. With its $\sim 40,000$ neurons belonging to around 70 different cell types, the medulla is thought to be the main processing center for color vision. Our lab has thoroughly characterized the different neuronal cell types in the medulla as well as projection and connectivity patterns. We are now in a position to analyze the function of different cell types in the medulla. In order to address this issue, we are currently establishing an assay for color vision which is based on the behavior of single flying flies in a colored LED arena under closed-loop conditions. We will take advantage of highly specific Gal4 drivers to manipulate neuronal activity in different medulla cell types to study their function in color vision. This approach will add functional data to the anatomical map of the Drosophila medulla and will provide insight into the processing of color information in the brain. 
VII.67

Effects of monochromatic light on the endogenous pacemaker of Drosophila melanogaster Corinna Wülbeck and Charlotte Helfrich-Förster Institute of Zoology, University of Regensburg

Corinna Wülbeck ${ }^{1}$, Charlotte Helfrich-Förster ${ }^{1}$

${ }^{1}$ Institut für Zoologie, Universität Regensburg

The fruit fly uses several photoreceptors to entrain the endogenous clock: The compound eyes, the HofbauerBuchner eyelets, the ocelli and cryptochrome (CRY). Recently, we showed that the compound eyes are crucial for the correct seasonal adaptation of morning (M) and evening (E) activity peaks. This includes the sensing of dim light and the acceleration and deceleration of $\mathrm{M}$ and E oscillators upon constant light. In the $c r y^{b}$ mutant background, constant white light provokes a simultaneous free-run of $\mathrm{M}$ - and E-oscillators with short and long periods, respectively. In a wild-type background such a differential action of constant light on $\mathrm{M}$ - and Eoscillators could hardly be seen, since CRY leads to degradation of TIM and stops the clock oscillations already at rather low irradiances. Here we show that this behavior is strongly dependent on the wavelength of light. Wavelengths in the range of 585-630 nm did not cause arrhythmia in wild-type flies even not at energies up to $4000 \mathrm{~mW} / \mathrm{cm}^{2}$. At these wavelengths we observe period changes and internal desynchronization into the above mentioned two activity components in an energy dependent manner. In contrast, light of $540 \mathrm{~nm}$ causes arrhythmia at energies as low as $85 \mathrm{~mW} / \mathrm{cm}^{2}$. This wavelength likely activates the blue light receptor CRY. We started a systematic test of monochromatic light to reveal which rhodopsin(s) of the compound eyes (Rh1, Rh3, Rh4, Rh5, Rh6) mediate the acceleration and deceleration of $\mathrm{M}$ - and E-oscillators, respectively. The first results will be presented.

\section{VII.68}

Genetic dissection of the neural circuitry for male courtship behavior

Daisuke Yamamoto ${ }^{l}$

${ }^{1}$ Division of Neurogenetics, Tohoku University Graduate School of Life Sciences

Drosophila melanogaster males display a highly stereotypic courtship ritual without any prior learning, and thus this behavior is considered to be initiated by the innate releasing mechanism (IRM). The fruitless (fru) gene plays a major role in the formation of the neural circuitry for this behavior. fru is a target of the female determinant protein Transformer (Tra), which ultimately leads to the male-specific presence of Fru protein in the nervous system. Thus, Fru specifies the sexual identity of neural cells, producing a sexual dimorphism of the neural circuitry. When tra is inactivated in females, these flies acquire the male-type neural circuitry and behave like males. By producing tra MARCM clones in the female brain, we determined the neuronal cluster which can initiate male courtship behavior when its sexual fate is transformed into the male. To determine the neural circuitry for male courtship behavior in its entirety, we examined the effects of inactivating different groups of neurons which express fru or putative mediators of pheromonal signals. The results of these experiments will be discussed in terms of the organizational principle of the IRM for male courtship behavior.

VII.69

Preferential temperature synchronization of the CRYnegative pacemaker neurons in the Drosophila circadian clock

Taishi Yoshii ${ }^{1}$, Charlotte Helfrich-Förster ${ }^{1}$

${ }^{1}$ Institute of Zoology, University of Regensburg

A blue light photo-receptive protein, Cryptochrome (CRY), plays an important role in light-synchronization of the circadian clock. In a former study, we found that not all of the $\sim 150$ pacemaker clock neurons express CRY. Therefore, we have speculated that the CRYpositive pacemaker neurons may be especially important for the light-entrainment, whereas the CRY-negative neurons may play a role in entrainment to other environmental cues, e.g. temperature. To investigate this hypothesis, we gave flies conflicting light-dark cycles (LD) and temperature cycles (TC) and recorded locomotor activity rhythms under these conditions. The locomotor rhythms of wild-type flies, CantonS, were strongly entrained to the LD. In contrast, cry-null mutant flies, cry ${ }^{\text {OUT }}$, synchronized to TC. This suggests that CRY keeps the clock to the light-entrainment.

TIMELESS (TIM) is one of clock components, which exhibits circadian rhythms in the protein levels in the pacemaker neurons. Double-immunostainings in CantonS flies using anti-TIM and anti-CRY antibodies revealed that the CRY-positive pacemaker neurons strongly synchronized to LD in the conflicting LD and TC, but most of CRY-negative neurons did neither synchronize to LD nor to TC. In $c r y{ }^{\text {OUT }}$ mutants, the TIM rhythm of most pacemaker neurons synchronized to TC. These results suggest the CRY-negative pacemaker neurons tend to prefer TC rather than LD. 
VII.70

\section{Where Humidity Will Guide Us Orientation Behavior of Drosophila Mutant Flies in a Humidity Gradient}

Bianca Zaepf ${ }^{1}$, Roland Strauss ${ }^{1}$

${ }^{1}$ Dept. of Zoology III-Neurobiology, Johannes Gutenberg-University, Mainz

The fly Drosophila melanogaster uses its hygrosensation to locate spots with comfortable humidity. Such humidity-dependent decision-making requires higher integration centers of the brain and likely also spatial orientation capacities. Sayeed and Benzer (1996) introduced a binary choice apparatus for testing Drosophila melanogaster in a simple humidity-choice paradigm. For the investigation of the humidity-based decision-making system of the fly we have started a screen for mutant lines aberrant in humidity-choice behavior. We have tested several mutant lines, which did not behave differently from wildtypestrains. In contrast, the mutant lines no-bridge ${ }^{K S 49}$, mushroom-bodies-deranged ${ }^{K S 65}$ and ellipsoid-bodyopen $^{678}$ have shown a significantly different humiditychoice behavior. These first results hint towards a role of the ellipsoid-body and/or the mushroom bodies in humidity orientation. Recently we study the flies' actual paths in an almost linear humidity-gradient. The change in relative humidity necessary to provoke the negative hydrotaxis in Drosophila melanogaster is from 66 to 76 percent relative humidity. The flies are capable of perceiving the direction of a humidity gradient and turn before the humidity can get from bad to worse. Last not least the choice for certain humidity has proven to be dependent on the current needs of the fly.

VII.72

Epistatic genetic networks control aggressive behavior in Drosophila.

Liesbeth Zwarts ${ }^{1}$, Alexis Edwards ${ }^{2}$, Mike Magwire ${ }^{3}$, Koen Norga ${ }^{4}$, Patrick Callaerts ${ }^{5}$, Trudy Mackay ${ }^{6}$, Robert Anholt ${ }^{6}$

${ }^{1}$ Lab of develomental genetics, VIB prj 8 \& KuLeuven, Belgium

2 Department of Genetics, W. M. Keck Center for Behavioral Biology, NCSU, Raleigh, NC, USA

${ }^{3}$ Institute of Evolutionary Biology, University of Edinburgh, Edinburgh, UK

${ }^{4}$ Lab of develomental genetics, VIB prj 8-KuLeuven, Children's Hospital KuLeuven, belgium

${ }^{5}$ Lab of develomental genetics, VIB prj 8-KuLeuven, belgium
6 Department of Genetics, W. M. Keck Center for Behavioral Biology, Department of Zoology, NCSU, Raleigh, NC, USA

Aggressive behavior mediates the establishment of stable social hierarchies. In human populations, however, it can carry a large socioeconomic burden. The genetic architecture underlying this behavior has not been extensively investigated. We measured aggression in a collection of co-isogenic $P$-element insertion lines of Drosophila melanogaster and selected 10 hyperaggressive lines with insertions at candidate genes that also show differential transcript abundance in response to artificial selection from a wild-derived population. None of these have been previously implicated in aggression. To assess to what extent they interact as functional ensembles we generated all 45 non-reciprocal double heterozygous F1 hybrids and identified a network of epistatic interactions involving 6 candidate genes. We also performed morphometric analyses of integrative brain structures, the mushroom bodies and ellipsoid body. We found that the genes, interacting epistatically at the level of behavior also have non-additive effects on morphometric parameters. Furthermore, the behavioral phenotype and the size of the ellipsoid body are correlated. Finally, transcriptional profiling using Affymetrix GeneChips of transheterozygotes that are part of the epistatic ensemble revealed coregulated genes with altered expression levels. This work presents a first step towards correlating genetic networks that predispose to aggression with the neural circuitry that mediates its manifestation.

VIII.1

BrainName: A controlled terminology system of the insect brain

Kei Ito ${ }^{1}$

${ }^{1}$ Institute of Molecular and Cellular Biosciences, University of Tokyo

VIII.2

Software tools for 3D registration and visualization of confocal stacks.

Benjamin Schmid ${ }^{l}$, Schindelin Johannes ${ }^{2}$, Cardona Albert $^{3}$, Heisenberg Martin ${ }^{1}$

${ }^{1}$ Department of Genetics and Neurobiology, University of Würzburg

${ }^{2}$ School of Psychology, University of St. Andrews

${ }^{3}$ Institute for Neuroinformatics, ETH Zurich 
Neuroanatomical studies in D. melanogaster often concern patterns of gene expression in the brain. 3D image stacks - acquired for instance by onfocal microscopy often are the raw data obtained in these experiments. However, their analysis suffers from the spatial variability of individual fly brains. To overcome this difficulty, a standard fly brain was proposed in the past providing a standardized coordinate system of the brain. To map an individual brain to this template, $3 \mathrm{D}$ image registration is necessary.

A. Jenett and J. Schindelin previously published the VIB protocol, a set of scripts for semi-automated image registration. These scripts however required the commercial software package Amira (Mercury Inc). We now ported this implementation for ImageJ, a free and open source image analysis software. In the field of biological and medical imaging.

In our opinion, ImageJ has the potential to replace several expensive and commercial software used in neuroanatomy today. Hundreds of developpers work on extensions which are open for everybody. One gap, however, is the lack of proper $3 \mathrm{D}$ visualization. We therefore developped a plugin, which allows to display image stacks optionally as surfaces, volume renderings or orthoslices. The software focusses on interactive, hardware-accelerated display of the stacks. Additionally, it offers a number of features, including volume editing, animation and movie recording.

VIII.4

Opposing effects of dietary protein and sugar regulate a transcriptional target of Drosophila insulin-like peptide signaling

Susanne Buch ${ }^{1}$, Christoph Melcher ${ }^{1}$, Michael J. Pankratz ${ }^{1}$

${ }^{1}$ Development and Genetics Unit, Universität Bonn

Metabolic regulation in higher organisms requires the interplay between the central nervous system and the peripheral organs. Specific neurosecretory cells of the Drosophila brain express insulin-like peptides (dilps), which have been shown to be important for various developmental and physiological processes, including larval growth, glucose homeostasis and aging. The genes in the periphery that mediate the physiological actions of the DILP-producing cells (IPCs) remain largely unknown. Through microarray analysis of flies in which the IPCs were ablated, we identified a target gene, target of brain insulin (tobi), which encodes an evolutionarily conserved alpha-glucosidase. Interestingly, tobi expression is increased by dietary protein and decreased by dietary sugar. This pattern is reminiscent of mammalian glucagon secretion, which is increased by protein intake and decreased by sugar intake, suggesting that tobi is regulated by a glucagon analogue. tobi expression is also eliminated upon ablation of neuroendocrine cells that produce adipokinetic hormone $(\mathrm{AKH})$, an analogue of glucagon. tobi is thus a novel peripheral target of the insulin- and glucagon-like signaling system that responds oppositely to dietary protein and sugar. The interplay of the insulin producing cells and the akh producing cells concernig the regulation of tobi demonstrates how brain neuroendocrine circuits modulate physiological processes and adapt metabolism to the given nutrient availability (1).

\section{The Role of Sleep in Learning of Drosophila}

Paul Shaw ${ }^{1}$

1 Anatomy and Neurobiology, Washington University School of Medicine

\section{LIST OF PARTICIPANTS}

Aberle, Hermann, aberleh@uni-muenster.de, University of Muenster, Institute for Neurobiology: V.6

Albertova, Viera, viera.albertova@biozentrum.uniwuerzburg.de, University of Würzburg, Genetics

Altenhein, Benjamin, balt@uni-mainz.de, University of Mainz, Institute of Genetics

Alves, Georges, georges.alves@u-bourgogne.fr, University of Burgundy, UMR CNRS 5548: VII.10

Anderson, David, wuwei@caltech.edu, Caltech/HHMI, Biology

Anderson, Peter, peter.anderson@ltj.slu.se, Swedish University of Agricultural Sciences, Chemical Ecology

Andrés, Marta, marta.andress@estudiante.uam.es, Autonoma University of Madrid, Department of Biology: II.11

Anthonis, Kristof, kristof.anthonis@med.kuleuven.be, KULeuven, Vesalius Research Center

Armstrong, J Douglas, jda@inf.ed.ac.uk, University of Edinburgh, School of Informatics: V.8

Aso, Yoshinori, aso@neuro.mpg.de, Max Planck Institute of Neurobiology, Behavioral Genetics: VI.11

Asztalos, Zoltan, zoltan@aktogen.com, Aktogen Ltd., University of Cambridge, Department of Genetics

Aust, Silvia, silvia.aust@rub.de, Ruhr Universität Bochum, AG Molekulare Zellbiochemie: V.10

Aydemir, Özkan, ozkanaydemir@gmail.com, University of Muenster, Institute of Neurobiology: III.5

Bai, Lei, lei.bai@yale.edu, Yale University, MCDB: III.6 
Bailey, Andrew, abailey@nimr.mrc.ac.uk, National Institute for Medical Research, Division of Developmental Neurobiology

Baines, Richard, Richard.Baines@manchester.ac.uk, University of Manchester, FLS

Balmer, Jasmin, Jasmin.Balmer@stud.unibas.ch, Biozentrum University of Basel: III.12

Bao, Sujin, sujin.bao@mssm.edu, Mount Sinai School of Medicine, Developmental and Regenerative Biology: II.9

Baumgardt, Magnus, magba@ifm.liu.se, Linköping University, Department of Clinical and Experimental Medicine: II.6

Bayersdorfer, Florian, florian.bayersdorfer@biologie. uni-regensburg.de, University of Regensburg, Zoology

Beatriz, Llamusi, beatrizllamusi@gmail.com, University of Valencia, Department of Genetics: I.11

Becker, Henrike, henribec@students.uni-mainz.de, Johannes Gutenberg Universität Mainz, Institut für Genetik: III.13

Bello, Bruno, bruno.bello@unibas.ch, Biozentrum University of Basel

Benito-Sipos, Jonathan, jonathan.benito@uam.es, Universidad Autónoma de Madrid: II.12

Benna, Clara, costast5@civ.bio.unipd.it, University of Padua, Department of Biology

Beramendi Heine, Ana, aberamendi@ice.mpg.de, Max Planck Institute for Chemical Ecology, Evolutionary Neuroethology

Berg, Christian, bergoron@web.de, Johannes Gutenberg-Universität Mainz, Institut für Zoologie III-Neurobiologie: VII.11

Berger, Christian, Christian.Berger@imba.oeaw.ac.at, IMBA: II.4

Berger, Sandra, sandra.berger@agroparistech.fr, MPI of Neurobiology, Axon guidance and Neuronal Connectivity Besse, Florence, besse@embl.de, EMBL, Developmental Biology

Bilen, Julide, julide.bilen@gmail.com, Janelia Farm Research Campus HHMI: VI.12

Birkholz, Oliver, birkholz@students.uni-mainz.de, Johannes Gutenberg-University Mainz, Institute of Genetics: III.14

Birman, Serge, birman@ibdml.univ-mrs.fr, CNRS-Université de la Méditerranée, IBDML-Developmental Biology Institute of Marseille-Luminy

Blau, Justin, justin.blau@nyu.edu, New York University, Biology Department: IV.P

Bloch, Alice, Alice.Bloch@gmx.de, University of Wuerzburg, Genetics: V.11

Bortnick, Rachel, rachel.bortnick@gmail.com, Dana Farber Cancer Institute, Harvard Medical School: VI.13

Bossing, Torsten, tb1o07@soton.ac.uk, University of Southampton, School of Biological Sciences
Botella, Jose A., jose.botella-munoz@biologie.uni-regensburg.de, University Regensburg, Lehrstuhl für Entwicklungsbiologie: I.4

Boto, Tamara, bototamara.uo@uniovi.es, University of Oviedo, Dep. Functional Biology: IV.6

Brand, Andrea,ahb@mole.bio.cam.ac.uk, University of Cambridge, The Gurdon Institute

Bredendiek, Nico, nico.bredendiek@gmx.de, Ruhr University, Bochum, Dept of cell physiology: III.15

Brembs, Björn, bjoern@brembs.net, FU Berlin, Neurobiologie: VII.12

Brierley, David, david.brierley@kcl.ac.uk, King's College London, MRC Centre for Developmental Neurobiology: VI.14

Briggs, Laura, 1b514@york.ac.uk, University of York, Biology: V.12

Brochtrup, Anna, annabrochtrup@gmx.de, WWU Münster, Institut f. Neurobiologie: VI.15

Buch, Susanne, susanne.buch@itg.fzk.de, Universität Bonn, Limes, Development and Genetics Unit: VIII.4

Buchner, Erich, buchner@biozentrum.uni-wuerzburg.de, University of Wuerzburg, Genetics and Neurobiology

Buescher, Marita, marita@tll.org.sg, Temasek Lifesciences Laboratory: II.13

Busch, Sebastian, sebastian.busch@biozentrum.uniwuerzburg.de, University of Wuerzburg: VI.16

Cachero, Sebastian, sebastian.cachero@mrc-lmb.cam.ac.uk, LMB-Medical Research Council, Neurobiology: VI.17

Calistri, Arianna, arianna.calistri@unipd.it, University of Padova, Department of Histology, Microbiology and Medical Biotechnologies

Callaerts, Patrick, patrick.callaerts@med.kuleuven.be, VIB, Laboratory of Developmental Genetics, K.U.Leuven, Department of Human Genetics

Canal, Macu, inmaculada.canal@uam.es, Universidad Autonoma Madrid, Department of Biology

Cantera, Rafael, rcantera@zoologi.su.se, Stockholm University, Zoology

Carlsson, Mikael, mikael.carlsson@zoologi.su.se, Stockholm University, Department of Zoology

Carmena, Ana, acarmena@umh.es, Instituto de Neurociencias de Alicante-CSIC/UMH, Developmental Neurobiology: II.2

Cavaliere, Sonia, sonia.cavaliere@bristol.ac.uk, University of Bristol, Department of Physiology and Pharmacology

Chaudhary, Kokil, kokil.chaudhary@biologie.uni-freiburg.de, University of Freiburg, Institute of Biology III: III.16

Chen, Chien-Kuo, gogockc@imb.sinica.edu.tw, Institute of Molecular Biology, Academia Sinica: V.3 
Chen, Ko-Fan, k.chen@qmul.ac.uk, Queen Mary, University of London, school of Biological and Chemical Science: VII.36

Chen, Yi-chun, chen.yi-chun@biozentrum.uni-wuerz burg.de, Universität Würzburg, Lehrstuhl für Genetik und Neurobiologie: VII.13

Cheng, Louise, lcheng@nimr.mrc.ac.uk, National institute for medical research, Developmental Neurobiology: III.17

Chia, William,wchia@tll.org.sg, Temasek Life Sciences Laboratory, National University of Singapore: II.P

Chotard, Carole, cchotar@nimr.mrc.ac.uk, National Institute for Medical Research, Molecular Neurobiology: III. 18

Christiansen-Engelhardt, Frauke, christiansen@virchow. uni-wuerzburg.de, Universität Würzburg, Rudolf Virchow Center for Experimental Biomedicine: V.13

Claridge-Chang, Adam, adam@well.ox.ac.uk, University of Oxford, Wellcome Trust Centre for Human Genetics

Clements, Jason, Jason.Clements@med.kuleuven.be, K.U.Leuven \& VIB, Dept. of Human Genetics: III.19

DasGupta, Shamik, shamik.dasgupta@umassmed.edu, University of Massachusetts Medical School, Worcester, Neurobiology: VIII.3

de Belle, Steven, steven.debelle@unlv.edu, University of Nevada, School of Life Sciences

Dekker, Teun, teun.dekker@ltj.slu.se, Swedish University of Agricultural Sciences, Dept. of Plant Protection Biology

Deng, Ying, ying.deng@rub.de, Ruhr University Bochum, Cell Physiology: IV.4

Dermaut, Bart, Bart.Dermaut@med.kuleuven.be, University of Leuven-VIB, Laboratory of Developmental Genetics

Diegelmann, Soeren,sd425@cam.ac.uk, University of Cambridge, Department of Zoology: VI.18

Domingos, Pedro, domingp@mail.rockefeller.edu, ITQB, Portugal/The Rockefeller University, USA: I.13

Döring, Frank, fdoering@mail.uni-wuerzburg.de, University of Wuerzburg, Institute of Physiology II

Drozdzecki, Andrzej, andrzej.drozdzecki@med. kuleuven.be, Katholike Universiteit Leuven, Department of Human Genetics

Dura, Jean-Maurice, jmdura@igh.cnrs.fr, Institute of Human Genetics, Department of Genetics\& Development: III.10

Dusik, Verena, v.dusik@gmx.de, University of Regensburg: VII.15

Eder, Sascha, seder@uni-mainz.de, University of Mainz, Institute for genetics: II.14

Edwards, Tara, edwardst@dal.ca, Dalhousie University, Biology/Neuroscience: V.2
Egger, Boris, b.egger@gurdon.cam.ac.uk, Wellcome Trust/Cancer Research UK, Gurdon Institute, University of Cambridge, Dept of Physiology, Development and Neuroscience: II.8

Ejima, Aki, aki@brandeis.edu, Brandeis University, Department of Biology: VI.5, VII.16

Elliott, Chris, cje2@york.ac.uk, University of York, Biology: V.14

Endo, Keita, kendo@iam.u-tokyo.ac.jp, The University of Tokyo, Institute of Molecular and Cellular Biosciences: II. 15

Enell, Lina, lina.enell@zoologi.su.se, Stockholm University, Zoology: VI.19

Engelen, Daniel, engelda@uni-muenster.de, University of Muenster, Institut of Neurobiology: III.20

Enneking, Eva-Maria, eva-maria.enneking@fmi.ch, Friedrich Miescher Institute for Biomedical Research, Neurobiology

Eschbach, Claire, claire.eschbach@stud-mail.uni-wuerz burg.de, University of Würzburg, Genetics and Neurobiology: VII.17

Estacio Gómez, Alicia, aestacio@cbm.uam.es, Universidad Autónoma de Madrid, Biología

Evers, Jan Felix, jfe22@cam.ac.uk, University of Cambridge, Zoology: VI.2

Fabrice, Lagasse, Fabrice.Lagasse@legs.cnrs-gif.fr, LEGS CNRS Gif sur Yvette: VII.18

Ferveur, Jean-François, jean-francois.ferveur@u-bour gogne.fr, CNRS/UNiversité de Bourgogne, Développement et Communication Chimique

Fiala, André, afiala@biozentrum.uni-wuerzburg.de, University of Wuerzburg, Department of Genetics and Neurobiology: VI.26, I.26, I.34

Fischbach, Karl-Friedrich, kff@uni-freiburg.de, AlbertLudwigs-University Freiburg, Institute for Biology III: III.23, III.11

Fouquet, Wernher, wernher.fouquet@virchow.uniwuerzburg.de, Bio-Imaging Center, Universität Würzburg, AG Sigrist: V.15

Fradkin, Lee, leef@lumc.nl, Leiden University Medical Center, Molecular Cell Biology

Franz, Mirjam, MirjamFranz@yahoo.de, Universisty of Wuerzburg, Department of genetics and neurobiology: I.7 Fricke, Robert, robertfricke@gmx.de, University of Münster, Institute of Neurobiology: V.17

Fukui, Ai, fukui@neuro.k.u-tokyo.ac.jp, University of Tokyo, Department of Physics: V.18

Garcia-Lopez, Amparo, amparo.garcia-lopez@uv.es, University of Valencia, Department of Genetics: I.14

Ge, Lihao, ge@nbio.uni-heidelberg.de, University of Heidelberg, Institute of Neurobiology (IZN)

Gendre, Nanaë, nanae.gendre@unifr.ch, University of Fribourg, Department of Biology 
Gerber, Bertram, bertram.gerber@biozentrum.uniwuerzburg.de, University of Wuerzburg

Giangrande, Angela, angela@ titus.u-strasbg.fr, IGBMCCNRS, ULP, Dept Cell and Dev Biology: III.4

Giannakou, Maria,meg37@cam.ac.uk, University of Cambridge, Department of Genetics

Gisselmann, Günter, guenter.gisselmann@rub.de, RuhrUniversität Bochum, Lehrstuhl für Zellphysiologie

Gmeiner, Florian, Florian.Gmeiner@biologie.uni-regensburg.de, Universität Regensburg, Lehrstuhl für Entwicklungsbiologie: I.15

Godenschwege, Tanja, godensch@fau.edu, Florida Atlantic Unversity, Biological Sciences: V.30

Goellner, Bernd, b.goellner@uni-muenster.de, Westfälische Wilhelms Universität Münster, Institut für Neurobiologie: V.19

Gohl, Christina, christinagohl@web.de, University of Münster, Institute for Neurobiology: V.20

Gomez-Diaz, Carolina, carolinag62@yahoo.es, Universidad de Oviedo, Functional Biology (Genetics): IV.7

Gonzalo, Alicia, alicia.gonzalo@uam.es, Autonoma University of Madrid, Departament of Biology: VII.19

Goossens, Tim, tim.goossens@med.kuleuven.be, K.U.Leuven/VIB, Lab of Developmental Genetics: III.22 Gopfert, Martin,m.gopfert@uni-koeln.de, University of Cologne, Inst. Zoology

Górska-Andrzejak, Jolanta, j.gorska-andrzejak@uj.edu.pl, Jagiellonian University, Cytology and Histology: V.21

Grabe, Veit, vgrabe@ice.mpg.de, Max Planck Institute for Chemical Ecology, Department of Evolutionary Neuroethology

Greiner, Birgit, birgit.greiner@biozentrum.uni-wuerzburg.de, University of Wuerzburg

Grunwald Kadow, Ilona, ikadow@neuro.mpg.de, Max Planck Institute of Neurobiology, Department of Molecular Neurobiology

Guillaume, Isabel, guillaume.isabel@espci.fr, ESPCICNRS: VII.27

Halder, Partho, partho.h@biozentrum.uni-wuerzburg.de, University of Wuerzburg, Genetics \& Neurobiology: V.22

Hampel, Stefanie, hampels@janelia.hhmi.org, HHMI, Janelia Farm Research Campus

Han, Kyung-An, kxh29@psu.edu, Pennsylvania State University, Biology: I.6

Han, Sungsik, sshan@korea.ac.kr, Korea university, cell engineering and 3D structure

Harada, Eriko, harada-eriko@ed.tmu.ac.jp, Tokyo Metropolitan University, Biological sciences: VII.21

Hardie, Roger, rch14@cam.ac.uk, University of Cambridge, Dept PDN
Hartmann, Beate, beate.hartmann@unibas.ch, Biozentrum, University of Basel

Hasan, Gaiti, gaiti@ncbs.res.in, National Centre for Biological Sciences, Genetics: IV.3

Hassan, Bassem, bassem.hassan@med.kuleuven.be, VIB Heindorf, Matthias, mheindorf@web.de, University of Heidelberg, Center for Neuroscience

Heisenberg, Martin, heisenberg@biozentrum.uniwuerzburg.de, Universität Würzburg, Genetik und Neurobiologie

Helfrich-Förster, Charlotte, charlotte.foerster@biologie.uni-regensburg.de, University of Regensburg, Department of Zoology: VII.7

Helmstädter, Martin, martin.helmstaedter@email.de, Universität Freiburg, AG Fischbach

Hidalgo, Alicia, a.hidalgo@bham.ac.uk, University of Birmingham, School of Biosciences: VI.1, III.21

Hiesinger, Peter Robin, robin.hiesinger@utsouthwestern.edu, UT Southwestern at Dallas, Department of Physiology

Hill, Julia, jah503@york.ac.uk, University of York, Department of Biology: VII.22

Hillebrand, Jens, jens.hillebrand@tcd.ie, Trinity College Dublin, Institute for Neuroscience: VI.21

Hindle, Samantha,sjh506@york.ac.uk, University of York, Biology: I.17

Hirth, Frank, Frank.Hirth@iop.kcl.ac.uk, MRC Centre for Neurodgeneration Research, King's College London

Ho, Thi Thu Cuc, hottcuc@yahoo.com, Ilsong Institute of Life Science, Hallym University, Molecular Neurogenetics Lab.: V.23

Hobbs, Brian, greenbhobbers@yahoo.com, University of Nevada Las Vegas, School of Life Sciences

Hodge, James, James.Hodge@bristol.ac.uk, University of Bristol, Department of Physiology and Pharmacology: IV.8

Hofbauer, Alois, alois.hofbauer@biologie.uni-regensburg.de, University of Regensburg, Institute for Zoology: VI.22

Holt, Matthew, mholt@gwdg.de, Max Planck Biophysical Chemistry, Neurobiology

Hosamani, Ravikumar, ravikumar_hosamani@rediffmail.com, Central Food Technological Research Institute, Biochemistry and Nutrition: I.18

Hovhanyan, Anna, ahovhanyan@gmail.com, MSZ/University of Wuerzburg, AG Raabe

Hoyer, Susanne, hoyer@biozentrum.uni-wuerzburg.de, University of Wuerzburg

Huang, Ju, Chinese Academy of Sciences, Institute of Neuroscience

Ibba, Irene, ireneibba@hotmail.com, University of Cagliari, Dep. Sperimental Biology, Sec. General Physiology: VII.23 
Inagaki, Hidehiko, hidehiko@caltech.edu, Caltech, Division of Biology: VII.25

Inoshita, Tsuyoshi, inorcb@gmail.com, University of Bourgogne

Isaac, Elwyn, r.e.isaac@leeds.ac.uk, University of Leeds, Faculty of Biological Sciences: VII.26

Ito, Kei, itokei@iam.u-tokyo.ac.jp, University of Tokyo, Institute of Molecular and Cellular Biosciences: VI.P, VIII.1

Itoh, Taichi Q, itorcb@gmail.com, Kyushu University, Dept. Biol., Grad. School Sci.,: VII.28

Izergina, Natalya, natalya.izergina@stud.unibas.ch, Biozentrum University of Basel: III.24

Jackson, Rob, rob.jackson@tufts.edu, Tufts University School of Medicine, Department of Neuroscience: VII.29 Jansen, Sebastian, jansens@uni-mainz.de, University of Mainz, Dep. of Genetics: II.17

Janssens, Els, elske_janssens@yahoo.com, KULeuven, CME, Laboratory of Developmental Genetics: I.19

Jauch, Mandy, mandy.jauch@gmx.de, Universtität Würzburg

Jeibmann, Astrid, jeibmann@uni-muenster.de, University hospital Münster, Institute of Neuropathology: I.20

Jenett, Arnim, jenetta@janelia.hhmi.org, HHMI Janelia Farm Research Campus, Rubin Lab: VI.23

Jiang, Yanrui, yanrui.jiang@molbio.uzh.ch, University of Zurich, Institute of Molecular Biology: III.2

Junfeng, Zheng, zjf075@gmail.com, Westfälische Wilhelms-Universität Münster, Institut für Neuro- und Verhaltensbiologie: VI.24

Juusola, Mikko,m.juusola@sheffield.ac.uk, Sheffield, Biomedical Science: VII.2

Kahsai Tesfai, Lily, lily.kahsai@zoologi.su.se, Stockholm University, Zoomorphology: VI.25

Kaiser, Andrea, kaiserandrea21@yahoo.de, University of Würzburg, Genetics and Neurobiology: I.21

Kallijärvi, Jukka, jukka.kallijarvi@helsinki.fi, University of Helsinki, Institute of Biotechnology: III.25

Kanellopoulos, Alexandros, kanellopoulos@fleming.gr, B.S.R.C “Al. Fleming', Institute of Molecular Biology and Genetics: VII.30

Kapustjanskij, Alexander, alexander.kapustjanskij@biozentrum.uni-wuerzburg.de, Universität Würzburg, Lehrstuhl für Genetik und Neurobiologie: VI.27

Karim, MD. Rezaul, rkarimcu@brain.riken.jp, RIKEN Brain Science Institute, Molecular Neuropathology Group: II.10

Karlsson, Daniel, dakar@ifm.liu.se, Linköping University, Department of Clinical and Experimental Medicine: II.5

Kaspar, Petra, petra.kaspar@uniklinikum-saarland.de, University of Heidelberg
Kato, Kentaro, katok@bham.ac.uk, The University of Birmingham, School of Biosciences: III.26

Kawasaki, Fumiko, fxk6@psu.edu, Penn State University, Departmen of Biology and Center of Molecular and Cellular Neuroscience: V.32

Keegan, Liam, Liam.Keegan@hgu.mrc.ac.uk, Medical Research Council UK, Human Genetics Unit, Western General Hospital: I.8

Khorramshahi, Omid, omid-k@gmx.de, RudolfVirchow-Zentrum, Bio-Imaging Center: IV.9

Kienitz, Bastian, basse2702@web.de, Johannes Gutenberg-Universität Mainz, Institut für Zoologie III-Neurobiologie: VII.31

Kim, A Young, kilenay@hanmail.net, Ilsong Institute of Life Science, Hallym University, Molecular Neurogenetics Lab.: V.26

Kistenpfennig, Christa, christa.kistenpfennig@biologie.uni-regensburg.de, Universität Regensburg, Institut für Zoologie: VII.32

Klämbt, Christian, klaembt@uni-muenster.de, University of Münster, Institut für Neuro- und Verhaltensbiologie

Klarsfeld, André, andre.klarsfeld@inaf.cnrs-gif.fr, CNRS, Institut de Neurobiologie A. Fessard, UPR2216: VII.33

Klug, Torsten, torsten.klug@itg.fzk.de, Universität Bonn, Development \& Genetics Unit

Knapek, Stephan, knapek@neuro.mpg.de, Max Planck Institute of Neurobiology: V.27

Kobler, Oliver, oliver.kobler@ifn-magdeburg.de, Leibniz Institute for Neurobiology, Neurochemistry and Molecular Biology: V.28

Koch, Marta, marta.koch@med.kuleuven.be, VIB and KULeuven, VIB Department of Molecular and Developmental Genetics: I.22

Kohsaka, Hiroshi, kohsaka@neuro.k.u-tokyo.ac.jp, University of Tokyo, Department of physics, Graduate School of Science: V.29

Kolodziejczyk, Agata, fichbio@yahoo.com, Stockholm University, Department of Zoology: VI.28

Krashes, Michael, michael.krashes@umassmed.edu, UMass Medical School, Neurobiology

Kraut, Rachel, rkraut@ibn.a-star.edu.sg, IBN/A-STAR: I.1

Kretzschmar, Doris, kretzsch@ohsu.edu, OHSU

Krishnan, Natraj, krishnan@science.oregonstate.edu, Oregon State University, Zoology: I.23

Krstic, Dimitrije, dimitrije.krstic@molbio.uzh.ch, University of Zurich, Institute for Molecular Biology: VI.29, VII.34, VII.9

Kuert, Philipp, philipp.kuert@stud.unibas.ch, Biozentrum, University of Basel 
Kumar, Vimlesh, kumarv@tcd.ie, University of Dublin Trinity College, Institute of Neuroscience: V.5

Kume, Kazuhiko,kkume@kumamoto-u.ac.jp, Kumamoto University, Inst. Molecular Embryology and Genetics: VII.35

Kummar, Abhilasha, abhilasha.kumar@stud.unibas.ch, Biozentrum, University of Basel: II.18

Kungl, Theresa, Theresa.Kungl@uni-wuerzburg.de, MSZ/University of Wuerzburg, AG Raabe

Kunz, Thomas, thkunz@students.uni-mainz.de, Johannes Gutenberg-Universität, Instiut für Genetik: II.19

Lahaye, Liza, L.L.Lahaye@lumc.nl, LUMC, Molecular Cell Biology: VI.30

Landgraf, Matthias, ml10006@cam.ac.uk, University of Cambridge, Department of Zoology

Langen, Marion, Marion.Langen@med.kuleuven.be, VIB/KULeuven, Developmental and Molecular Genetics Section: VI.31

Laridon, Bram, bram.laridon@med.kuleuven.be, University of Leuven, Laboratory of Developmental Genetics: I.24

Larsch, Johannes, johannes@larsch.com, University of Konstanz: VII.37

Lavista, Sofia, slavista-llanos@ice.mpg.de, Max Planck Institute for Chemical Ecology, MPI-Evolutionary Neuroethology: VI.32

Lee, Dae-Weon, 71dwlee@hanmail.net, Ilsng Institute of Life Science, Hallym University, Molecular Neurogenetics Lab.: V.31

Lehmann, Fritz-Olaf, fritz.lehmann@uni-ulm.de, University of Ulm, Neurobiology

Leisibach, Basil, basil.leisibach@unifr.ch, University of Fribourg, Department of Biology

Leiss, Florian, fleiss@neuro.mpg.de, Max Planck Institute of Neurobiology, Tavosanis Lab: VI.6

Leptourgidou, Flora, leptourgidou@fleming.gr, BSRC Alexander Fleming, Institute of Molecular Biology and Genetics: VII. 38

Liedel, Stefanie, stefanie.liedel@stud-mail.uni-wuerzburg.de, Universität Würzburg

Lin, Wei-Hsiang, d864611@life.nthu.edu.tw, University of Manchester, Faculty of Life Sciences: IV.5

Lindström, Riitta, riitta.lindstrom@helsinki.fi, University of Helsinki, Institute of Biotechnology: I.25

Linneweber, Gerit, gerit.linneweber@biologie.uni-freiburg.de, Albert-Ludwigs-University Freiburg, Neurogenetics: II.21

Loncle, Nicolas, nicolas.loncle@kcl.ac.uk, King's College London, Developmental Neurobioloy

López Arias, Begoña, begonna.lopez@uam.es, Universidad Autónoma de Madrid, Biology: VII.39

Losada, María, maria.losada@uam.es, Universidad Autónoma de Madrid, Biology: III.27
Louis, Matthieu,mlouis@crg.es, Centre for Genomic Regulation, Systems Biology Unit: VII.40

Luan, Haojiang, luanh@mail.nih.gov, NIMH: VI.33

Ludlow, Zoe, zoe.ludlow@iop.kcl.ac.uk, Institute of Psychiatry, King's College London, MRC Centre for Neurodegeneration Research: II.22

Lüer, Karin, lueer@uni-mainz.de, University of Mainz, Institute of Genetics

Maier, Dieter,maierdie@uni-hohenheim.de, Universität Hohenheim, Institut für Genetik (240): II.23

Malenica, Ana-Marija, malenica@neuro.mpg.de, MPI Neurobiology

Manière, Gérard, gerard.maniere@u-bourgogne.fr, University of Burgundy, UMR CNRS 5548

Mann, Klaudiusz, kmann@neuro.mpg.de, Max Planck Institute of Neurobiology, Axonal Guidance and Neuronal Connectivity

Marley, Richard, r.marley@manchester.ac.uk, University of Manchester: VI.34

Martin, Jean-Rene, jean-rene.martin@inaf.cnrs-gif.fr, CNRS, NBCM, UPR-9040

Masek, Pavel, flytaste@gmail.com, University of California at Berkeley, Scott Lab: VII.41

Masuda-Nakagawa, Liria, almasuda@mail.ecc.u-tokyo.ac.jp, University of Tokyo, Institute of Molecular and Cellular Biosciences: VI.7

Mauss, Alex, asm54@cam.ac.uk, University of Cambridge, Department of Zoology: VI.35

Mayer, Lisa,mayerlis@students.uni-mainz.de, University of Mainz

Mayor, Ugo, um203@cam.ac.uk, University of Cambridge, WT CR UK Gurdon Institute: I.27

McCann, Cathal, cmccann2@tcd.ie, Trinity College Dublin, Dept of Genetics/Institute of Neuroscience

McKellar, Claire,mckellarc@janelia.hhmi.org, HHMI/ Janelia Farm Research Campus: VI.36

Mehnert, Kerstin, kerstin.mehnert@zoologi.su.se, Stockholm University, Zoology: V.33

Meinertzhagen, Ian, iam@dal.ca, Dalhousie University, Psychology and Neuroscience: VI.50

Melzer, Juliane, juliane.melzer@uni-wuerzburg.de, University of Wuerzburg, Institut für Medizinische Strahlenkunde und Zellforschung

Mentzel, Benjamin, benjamin.mentzel@mail.uni-wuerz burg.de, Universität Würzburg, Institut für medizinische Strahlenkunde und Zellforschung

Michels, Birgit, birgit.michels@biozentrum.uni-wuerzburg.de, University of Wuerzburg, Department of Genetics and Neurobiology: VII.42

Mihaly, Jozsef, mihaly@brc.hu, Biological Research Center, Hungarian Academy of Sciences, Institute of Genetics 
Milton, Valerie, vjm500@york.ac.uk, University of York, Biology

Minocha, Shilpi, shilpi.minocha@molbio.uzh.ch, University of Zürich, Institute of Molecular Biology: III.9

Mishra, Dushyant, dushyant.mishra@biozentrum.uniwuerzburg.de, Universität Würzburg, Lehrstuhl für Genetik und Neurobiologie: VII.43

Miskiewicz, Katarzyna, Katarzyna.Miskiewicz@med. kuleuven.be, VIB, K.U.Leuven, Department of Molecular and Developmental Genetics, Center for Human Genetics: V.34

Miyazaki, Takaaki, tm@cb.k.u-tokyo.ac.jp, The University of Tokyo, Institute of Molecular and Cellular Biosciences: VI.37

Monedero, Ignacio, ignacio.monedero@estudiante. uam.es, Universidad Autonoma de Madrid, Biology: I.29 Moore, Adrian, adrianm@brain.riken.jp, RIKEN Brain Science Institute, Molecular Neuropathology Group

Moressis, Anastasios, moressis@fleming.gr, BSRC Alexander Fleming, Institute of Molecular Biology and Genetics: VII.44

Münch, Daniel, daniel.muench@uni-konstanz.de, Universität Konstanz, FB Biologie, AG Galizia

Muñoz-Soriano, Veronica, veronica.munoz-soriano@ uv.es, University of Valencia, Department of Genetics: I.30

Murakami, Satoshi, smurakami@iam.u-tokyo.ac.jp, University of Tokyo, Institute of Molecular and Cellular Biosciences: VII.45

Murmu, Meena, meena.murmu@inaf.cnrs-gif.fr, CNRS UPR-9040, Laboratoire de Neurobiologie Cellulaire et Moléculaire: VI.38

Nässel, Dick, dnassel@zoologi.su.se, Stockholm University, Zoology

Navarro Langa, Juan Antonio, juan.navarro@biologie.uni-regensburg.de, University of Regensburg, Lehrstuhl für Entwicklungsbiologie: I.31

Negele, Julia, negele@neuro.mpg.de, MPI of Neurobiology, Molecular Neurobiology: III.28

Neuhaus, Eva, eva.neuhaus@rub.de, Ruhr-Universitaet Bochum, Cell Physiology

Neuser, Kirsa, kirsa.neuser@biozentrum.uni-wuerz burg.de, University Mainz, Institute of Zoology III, Neurobiology: VII.46

Nicolaï, Laura, laura.nicolai@med.kuleuven.be, KULeuven VIB, Laboratory of Neurogenetics

Niewalda, Thomas, thomas.niewalda@biozentrum.uniwuerzburg.de, Universität Würzburg, Department of Genetics and Neurobiologie: VII.47

Noll, Markus, markus.noll@molbio.uzh.ch, University of Zürich, Institute for Molecular Biology

North, Annemarie, at288@hermes.cam.ac.uk, University of Cambridge, Department of Zoology: III.29
Nose, Akinao, nose@k.u-tokyo.ac.jp, University of Tokyo, Graduate School of Frontier Sciences

Nuwal, Tulip, tulip@biozentrum.uni-wuerzburg.de, University of Wuerzburg, Genetics and Neurobiology: V.35

Occhi, Simona, occhi.simona@hsr.it, Dulbecco Telethon Institute, DIBIT-San Raffaele Scientific Institute, Developmental neurobiology: I.9

O'Hare, Megan, megano_hare@hotmail.com, Kings College London, MRC Centre for Developmental Neurobiology

Ohler, Stephan, ohler@neuro.mpg.de, Max Planck Institute of Neurobiology, Axonal Guidance and Neuronal Connectivity: III.30

Ohmann, Elisabeth, elisabeth.ohmann@biologie.uniregensburg.de, Universität Regensburg, Institut für Zoologie

O'Kane, Cahir, c.okane@gen.cam.ac.uk, University of Cambridge, Department of Genetics

Oliva, Carlos, coliva@med.uchile.cl, Universidad de Chile, Institute for Biomedical Sciences: VI.39

Ordway, Richard, rwo4@psu.edu, Penn State University, Department of Biology and Center for Molecular and Cellular Neuroscience: V.24

Orihara-Ono, Minako, orihara@sc.itc.keio.ac.jp, Keio Univ., Sch. Med., Dept. Physiology: II.24

Oswald, Matthew, mo501@york.ac.uk, The University of York: V.36

Owald, David, owald@virchow.uni-wuerzburg.de, University of Würzburg, Rudolf Virchow Center: V.37

Papanikolopoulou, Aikaterini, papanikolopoulou@ fleming.gr, BSRC Alexander Fleming: I.32

Park, Se Jin, fufujini@korea.ac.kr, Korea university, Graduate school of life science and biotechnology

Parmentier, Marie-Laure, marie-laure.parmentier@ igf.cnrs.fr, Institut de Génomique Fonctionnelle, Department of Neurobiology

Partridge, Linda, 1.partridge@ucl.ac.uk, University College London, Institute of Healthy Ageing, Dept of Genetics, Evolution and Environment: I.P

Pauls, Dennis, dennis.pauls@unifr.ch, University of Fribourg, Department of Biology: VII.48

Peters, Marc, marc.peters@itg.fzk.de, University of Bonn-LIMES, Development \& Genetics Unit: VI.40

Petrova, Iveta, I.M.Petrova@lumc.nl, LUMC, MCB: VI.41

Petrovic, Milan,m.petrovic@uni-muenster.de, Universität Münster, Institut für Neurobiologie: VI.42

Pettersson, Nina, nina.pettersson@izb.unibe.ch, University of Bern, Institute of Cellbiology': I.33

Pfitzenmaier, Johanna, johanna.pfitzenmaier@unifr.ch, University of Fribourg, Department of Biology

Pflugfelder, Gert O., pflugfel@uni-mainz.de, Universität Mainz 
Pielage, Jan, jan.pielage@fmi.ch, Friedrich Miescher Institute for Biomedical Research, Neurobiology

Pilgram, Gonneke, g.s.k.pilgram@lumc.nl, Leiden University Medical Centre, MCB/NOB: V.38

Pokrzywa, Malgorzata, malgorzata.pokrzywa@molbiol. umu.se, Linkoping University, Dept of Clinical and Experimental Medicine

Potikanond, Saranyapin, s.potikanond@lumc.nl, Leiden University Medical center, molecular cell biology: V.39

Preat, Thomas, thomas.preat@espci.fr, ESPCI-CNRS

Preiss, Anette, preiss@uni-hohenheim.de, Universität Hohenheim, Institut für Genetik (240)

Prieto Godino, Laura Lucia, llp23@cam.ac.uk, University of Cambridge, Department of Zoology: VI.43

Prokop, Andreas, Andreas.Prokop@manchester.ac.uk, The University of Manchester, Faculty of Life Sciences: V.P

Pütz, Stefanie, neffi2@gmx.de, Ruhr-Universität Bochum, AG Moelkulare Zellbiochemie

Pyza, Elzbieta, elzbieta.pyza@uj.edu.pl, Institute of Zoology/Jagiellonian University, Deaprtment of Cytology and Histology

Raabe, Thomas, thomas.raabe@mail.uni-wuerzburg.de, University of Wuerzburg, Medical Radiation and Cell Research

Ramaswami, Mani, mani@u.arizona.edu, Trinity College Dublin, TCIN and Genetics

Randlkofer, Barbara, b.randlkofer@gmx.net, Swedish University of Agricultural Sciences (SLU), Division of Chemical Ecology, Dept. of Plant Protection: VII.50

Rasse, Tobias, tobias.rasse@medizin.uni-tuebingen.de, University of Tuebingen, Cellular Neurology: V.25

Reaume, Christopher, christopher.reaume@utoronto.ca, University of Toronto, Ecology and Evolutionary Biology: VII.5

Reeve, Simon, simon.reeve@well.ox.ac.uk, Oxford University, Wellcome Trust Centre for Human Genetics

Reichert, Heinrich, heinrich.reichert@unibas.ch, University of Basel, Biozentrum

Richter, David, metatron_1998@yahoo.com, LIMES/ University Bonn, Development and Genetics unit

Rickert, Christof, rickert@uni-mainz.de, University of Mainz, Institute of Genetics: III.31

Rieger, Dirk, dirk.rieger@biologie.uni-regensburg.de, University of Regensburg, Institute of Zoology: VII.51

Riemensperger, Thomas, riemensperger@ibdml.univmrs.fr, CNRS-Université de la Méditerranée, IBDMLDevelopmental Biology Institute of Marseille-Luminy: VII.8, I.5

Rister, Jens, jr190@nyu.edu, New York University, NYU, Department of Biology, Laboratory for Molecular Genetics
Robinson, Iain, iain.robinson@pms.ac.uk, University of Plymouth, Peninsula Medical School: V.40

Rodrigues, Floriano, floriano@uni-muenster.de, Westfälische Wilhelms-Universität Münster, Institut für Neuro- und Verhaltensbiologie: III.32

Rogulja-Ortmann, Ana, rogulja@uni-mainz.de, University of Mainz, Institute of Genetics: II.7

Roman, Gregg, gwroman@uh.edu, University of Houston, Dept. Biology and Biochemistry: VII.52

Rouyer, François, rouyer@inaf.cnrs-gif.fr, CNRS, UPR2216, INAF: VII.14

Sachse, Silke, ssachse@ice.mpg.de, Max Planck Institute for Chemical Ecology, Department of Evolutionary Neuroethology

Saito, Mai, saito@ibdml.univ-mrs.fr, CNRS-Université de la Méditerranée, IBDML-Developmental Biology Institute of Marseille-Luminy: V.42

Salecker, Iris, isaleck@nimr.mrc.ac.uk, National Institute for Medical Research, Division of Molecular Neurobiology: III.P

Sareen, Preeti, preeti.sareen@biozentrum.uni-wuerz burg.de, Universität Würzburg, Lehrstuhl für Genetik und Neurobiologie: VII.53

Sartori, Elena, elena.sartori@unipd.it, University of Padova, Department of Histology, Microbiology and Medical Biotechnologies

Saumweber, Timo, timo.saumweber@biozentrum.uniwuerzburg.de, University of Wuerzburg, Genetics and Neurobiology: VII.54

Schenck, Annette, a.schenck@antrg.umcn.nl, Nijmegen Centre for Molecular Life Science, Radboud University Nijmegen Medical Center, Department of Human Genetics: I.12

Scheper, Christoph, christoph-t.scheper@web.de, Universität Münster, Institut für Neurobiologie: VI.3

Scherzer, Roni, roni.scherzer@gmail.com, Tel-Aviv University, Biotechnology: I.3

Schindelin, Johannes, johannes.schindelin@gmx.de, unaffiliated

Schlieder, Marlen, Marlen.Schlieder@med.kuleuven.be, VIB (KU Leuven)

Schmid, Benjamin, bene.schmid@gmail.com, University of Würzburg, Department of Genetics and Neurobiology: VIII.2

Schmidt, Imke, schmimke@uni-muenster.de, University of Muenster, Institute of Neurobiology: III.33

Schnaitmann, Christopher, schnaitmann@neuro.mpg. de, Max-Planck-Institute of Neurobiology: VII.55

Schneider, Andrea, S.Keksi@web.de, University of Würzburg, Genetics and Neurobiology: I.35

Schneider, Martina, maschneider@bio.ku.dk, University of Copenhagen, Dept of Biology 
Schnell, Bettina, schnell@neuro.mpg.de, Max Planck Institute of Neurobiology, Department of Systems and Computational Neurobiology: VI.44

Schneuwly, Stephan, stephan.schneuwly@biologie. uni-regensburg.de, University of Regensburg, Department of Zoology

Scholz, Henrike, henrike.scholz@biozentrum.uni-wuerz burg.de, University of Wuerzburg, Genetics and Neurobiology

Schultz, Sebastian, sebsc@ibk.liu.se, University of Linköping, Department of Clinical and Experimental Medicine: I.36

Schuster, Christoph, Schuster@nbio.uni-heidelberg.de, Interdisciplinary Center for Neurosciences IZN of the University of Heidelberg, Neurobiology

Schützner, Peter, peter.schuetzner@uni-ulm.de, University of Ulm, Neurobiology: VII.56

Seeds, Andrew, seedsa@janelia.hhmi.org, Howard Hughes Medical Institute, Janelia Farm Research Campus: VII.6

Segal, Daniel, dsegal@post.tau.ac.il, Tel-Aviv University, Dept. Molecular Microbiology \& Biotechnology

Seibert, Janina, seiberj@uni-mainz.de, University of Mainz, Institute of Genetics: II.25

Sejourne, Julien, Julien.Sejourne@espci.fr, ESPCI, GDSM: VII.3

Seki, Yoichi, yseki@ice.mpg.de, Max Planck Institute for Chemical Ecology, Department of Evolutionary Neuroethology

Selcho, Mareike, mareike.selcho@unifr.ch, University of Fribourg, Department of Biology: VII.57

Semenov, Eugene, esen@obzor.bio21.bas.bg, Institute of Molecular Biology: IV.10

Sen, Sonia, sonia@ncbs.res.in, National Centre for Biological Sciences., Genetics and Development: II.26

Serway, Christine, serwayc@unlv.nevada.edu, University of Nevada Las Vegas, School of Life Sciences: VII.4 Shaw, Paul, shawp@pcg.wustl.edu, Washington University School of Medicine, Anatomy and Neurobiology

Shcherbakova, Oksana, oksana_kysla@yahoo.com, Lviv National University of Ivan Franko, Department of Genetics and Biotechnology: I.37

Shinomiya, Kazunori, k-shino@cb.k.u-tokyo.ac.jp, University of Tokyo, Institute of Molecular and Cellular Biosciences: VI.45

Shirras, Alan, a.shirras@lancaster.ac.uk, University of Lancaster, Dept. of Biological Sciences

Sierralta, Jimena, jimena@neuro.med.uchile.cl, Univer sidad de Chile, Institute for Biomedical Sciences

Sigrist, Stephan, stephan.sigrist@virchow.uni-wuerz burg.de, Universität Würzburg

Silies, Marion,msilies@uni-muenster.de, Institute for Neurobiology, University of Münster: III.3
Sinakevitch, Irina, sinakevitch@ibdml.univ-mrs.fr, CNRS-Université de la Méditerranée, IBDML-Deve lopmental Biology Institute of Marseille-Luminy: VI.46, V.9

Singhal, Nidhi, nidhi@biozentrum.uni-wuerzburg.de, Universität Würzburg, Genetics and Neurobiology: VII.58

Sivdasan, Rajeeve, rajivshivan@gmail.com, Universität Würzburg

Skoulakis, Efthimios, skoulakis@fleming.gr, BSRC Alexander Fleming, Institute of Molecular Biology and Genetics: VII.20

Söderberg, Jeannette, jeannette.soderberg@zoologi. su.se, University of Stockholm, Department of Zoology: VI.47

Solanki, Narendra, narendra@biozentrum.uni-wuerz burg.de, University of Würzburg, Institute of Genetics \& Neurobiology: VII.59

Soller, Matthias,m.soller@bham.ac.uk, University of Birmingham, School of Biosciences: V.4

Southall, Tony, tds29@cam.ac.uk, Universitu of Cambridge, Gurdon Institute: II.3

Speder, Pauline, p.speder@gurdon.cam.ac.uk, University of Cambridge

Spindler, Shana, sfawcett@ucla.edu, University of California, Los Angeles: VI.48

Spletter, Maria, spletter@stanford.edu, Stanford University, Biology: VI.4

Sprecher, Simon, simon.sprecher@gmail.com, New York University, Departement of Biology: III.8

Stanewsky, Ralf, r.stanewsky@qmul.ac.uk, Queen Mary, University of London, SBCS: VI.20

Stark, Felix, Felix.Stark@mail.uni-wuerzburg.de, MSZ/ University of Wuerzburg, AG Raabe: VI.49

Stephan, Daniel, dstephan@neuro.mpg.de, MPI of Neurobiology, Sensory Neurogenetics

Stephan, Raiko, raiko.stephan@uni-muenster.de, University of Münster, Institute for Neurobiology

Stocker, Reinhard, reinhard.stocker@unifr.ch, University of Fribourg, Department of Biology

Storkebaum, Erik, erik.storkebaum@med.kuleuven. be, VIB \& KULeuven, Laboratory of Developmental Genetics: I.38

Störtkuhl, Klemens, klemens.stoertkuhl@rub.de, University of Bochum, Biology and Biotechnology: VII.60

Strausfeld, Nicholas, flybrain@neurobio.arizona.edu, University of Arizona, Neurobiology

Strauss, Roland, rstrauss@uni-mainz.de, Johannes Gutenberg-Universität Mainz, Institut für Zoologie IIINeurobiologie

Strutz, Antonia, astrutz@ice.mpg.de, Max Planck Institute for Chemical Ecology, Department of Evolutionary Neuroethology 
Suzuki, Takashi, suzukit@neuro.mpg.de, Max Planck Institute of Neurobiology, Axonal Guidance and Neuronal Connectivity: III.7

Sweeney, Sean, sts1@york.ac.uk, University of York, Department of Biology (Area 9): I.28

Tabata, Tetsuya,ttabata@iam.u-tokyo.ac.jp, University of Tokyo

Tabone, Christopher, tabonec@unlv.nevada.edu, University of Nevada, Las Vegas: VII.61

Tanaka, Nobuaki, tanakano@mail.nih.gov, NIH, USCNE: VI.8

Tavosanis, Gaia, gaia@neuro.mpg.de, MPI of Neurobiology, Molecular Neurobiology

Tear, Guy, guy.tear@kcl.ac.uk, King’s College, London, MRC Centre for Developmental Neurobiology

Technau, Gerhard, technau@uni-mainz.de, University of Mainz, Institute of Genetics

Thakurdas, Pooja, pooja.thakurdas@yahoo.co.in, University of Regensburg, Institute of Zoology

Thoma, Michael, michael.thoma@uni-konstanz.de, University of Konstanz: VI.51

Thomas, Jean Louis, drosophiliste@yahoo.fr, university of heidelberg, IZN AG Schuster

Thomas, Ulrich, thomas@ifn-magdeburg.de, Leibniz Institute for Neurobiology, Neurochemistry and Molecular Biology

Thor, Stefan, steth@ifm.liu.se, Linkoping University, Dept of Clinical and Experimental Medicine

Thum, Andreas, andreas.thum@unifr.ch, University of Fribourg, Department of Biology: VII.62

Timinszky, Gyula, timinszk@embl.de, EMBL Heidelberg, Gene Expression Unit

Timofeev, Katarina, katarina.t@uni-muenster.de, WWU Muenster, Institut fuer Neurobiologie: VI.52

Tomita, Jun, tomita@kumamoto-u.ac.jp, Kumamoto University, Institute of Molecular Embryology and Genetics: VII.63

Torroja, Laura, laura.torroja@uam.es, Universidad Autónoma de Madrid, Biology

Triphan, Tilman, tilman.triphan@biozentrum.uni-wuerz burg.de, Johannes Gutenberg-Universität Mainz, Institut für Zoologie III-Neurobiologie: VII.64

Turner, Ashby, ashby.turner@uky.edu, University of Kentucky, Biology: VI.53

Tuxworth, Richard, richard.tuxworth@kcl.ac.uk, Kings College London, MRC Centre for Developmental Neurobiology: I.39

Ueno, Taro, taro.ueno@gmail.com, University of Kumamoto: VII.65

Uhlenbrock, Guido, Guido.Uhlenbrock@rub.de, Ruhr Universität Bochum, AG Molekulare Zellbiochemie: V.41
Urban, Joachim, jurban@uni-mainz.de, University of Mainz, Institute of Genetics

Uytterhoeven, Valerie, valerie.uytterhoeven@med. kuleuven.be, University of Leuven, VIB, Dept. of Molecular and Developmental Genetics: V.44

van den Brink, Daan, daan.vdbrink@kcl.ac.uk, King's College London, MRC Centre for Developmental Neurobiology: VI.54

Verstreken, Patrik, patrik.verstreken@med.kuleuven.be, VIB and KULeuven, Molecular and Developmental Genetics and Center for Human Genetics: I.16

Vogt, Nina, nv16@nyu.edu, New York University, Biology: VII.66

von Alpen, Desirée, desireecatherine.vonalpen@unifr.ch, University of Fribourg, Department of Biology

von Essen, Alina, alina.vonessen@unifr.ch, University of Fribourg, Department of Biology

von Hilchen, Christian, vonhilch@uni-mainz.de, University of Mainz, Institute of Genetics: III.34

von Trotha, Jakob, jwv20@cam.ac.uk, University of Cambridge, The Wellcome Trust/Cancer Research UK Gurdon Institute: III.35

Waddell, Scott, scott.waddell@umassmed.edu, University of Massachusetts Medical School, Neurobiology

Wagh, Dhananjay, dwagh@stanford.edu, Stanford University

Wang, Cheng, gmswc@nus.edu.sg, DUKE-NUS Graduate Medical School: II.27

Wang, Jing, jw800@ucsd.edu, University of CaliforniaSan Diego, Division of Biological Sciences: V.7

Wardill, Trevor, t.wardill@shef.ac.uk, University of Sheffield, Department of Biomedical Science: VI.56

Wegener, Christian, wegener@staff.uni-marburg.de, Philipps-University, Animal Physiology, Dept. of Biology: VI.57, VI.55

Wegener, Stephanie, stephanie.wegener@gmx.net, University of Würzburg

Weiss, Linnea, linnea.weiss@yale.edu, Yale University, Mol, Cell \& Dev Biology: VI.9

Wentzell, Jill, wentzell@ohsu.edu, Oregon Health \& Science University: I.2

Wessnitzer, Jan, jwessnit@inf.ed.ac.uk, University of Edinburgh: VI.58

White, Benjamin, benjaminwhite@mail.nih.gov, National Institute of Mental Health, USA, Laboratory of Molecular Biology: VII.49

White, Katherine, katherine.white@iop.kcl.ac.uk, Institute of Psychiatry, King's College London, MRC Centre for Neurodegeneration Research: I.40

Whitworth, Alex, a.whitworth@sheffield.ac.uk, University of Sheffield, Biomedical Science

Winther, Åsa, asa.winther@zoologi.su.se, Stockholm University, Department of Zoology: VII.24 
Wise, Alexandria, awise.ccny@gmail.com, City College of New York, Biology

Wolf, Reinhard, reinhard.wolf@biozentrum.uni-wuerz burg.de, Universität Würzburg, Lehrstuhl für Genetik und Neurobiologie

Wolfram, Verena, Verena.Wolfram@manchester.ac.uk, Manchester University, Faculty of Life Science/Neuroscience: VI.59

Wu, Chun-Fang, chun-fang-wu@uiowa.edu, University of Iowa, Department of Biology: I.41, V.43, IV.2

Wülbeck, Corinna, corinna.wuelbeck@biologie.uniregensburg.de, Universität Regensburg, Institut für Zoologie: VII.67

Yamamoto, Daisuke, daichan@mail.tains.tohoku.ac.jp, Tohoku University Graduate School of Life Sciences, Division of Neurogenetics: VII.68

Yarali, Ayse, ayse.yarali@biozentrum.uni-wuerzburg.de, Universitaet Wuerzburg, Lehrstuhl Genetik und Neurobiologie

Yasugi, Tetsuo, yasugi@iam.u-tokyo.ac.jp, University of Tokyo, IMCB: II.28

Yoshii, Taishi, x100dc@netscape.net, University of Regensburg, Institute of Zoology: VII.69

Young, Joanna, J.Young@ed.ac.uk, University of Edinburgh: VI.60

Yuva Aydemir, Yeliz, yuva@uni-muenster.de, University of Muenster, Institute of Neurobiology: III.36

Zaepf, Bianca,zaepf@uni-mainz.de, Johannes Gutenberg-University, Mainz, Dept. of Zoology III-Neurobiology: VII.70

Zars, Troy, zarst@missouri.edu, University of Missouri, Division of Biological Sciences: VII.71

Ziegler, Anna, anna_ziegler@web.de, Ruhr Universität Bochum, Chemistry and Biochemistry: V.45

Zwarts, Liesbeth, liesbeth.zwarts@med.kuleuven.be, KuLeuven: VII.72

\section{LIST OF AUTHORS}

Aberle, Hermann: V.6, V.19

Aburatani, Hiroyuki: V.18

Acharjee, Shaona: V.32

Ackerson, Larry C.: V.41

Adrian, W. Moore: II.10

Ahmad, Margaret: VII.7

Aigaki, Toshiro: VII.21

Albert, Cardona: VIII.2

Albert, Jörg T.: I.26

Alcorta, Esther: IV.6, IV.7

Alla, Suneel Reddy: V.5

Altenhein, Benjamin: III.34
Alvarez-Abril, M.Carmen: I.14

ALVES, Georges: VII.10

Anderson, Peter: VII.50

Andlauer, Till Felix Malte: III.11

Andreas, Schmid: IV.9

Andrés, Marta: II.11, III.27

Angioy, AnnaMaria: VII.23

Anholt, Robert: VII.72

Armstrong, Douglas: VI.60

Armstrong, J Douglas: V.8

Artero, Ruben: I.14

Aso, Yoshinori: VI.11

Aust, Silvia: V.10

Ayaz, Derya: I.22

Aydemir, Özkan: III.5

Bachmann, Andre': V.28

Bai, Lei: III.6

Baines, Richard: V.38, VI.59

Baines, Richard A.: IV.5

Balmer, Jasmin: III.12

Banovic, Daniel: V.19

Bao, Sujin: II.9

Barbee, Scott: VI.21

Bate, Michael: VI.2, VI.18, VI.43

Baumgardt, Magnus: II.5, II.6, II.12

Bayersdorfer, Florian: I.4

Beatriz, Llamusi: I.11

Becker, Henrike: III.13

Beckervordersandforth, Ruth: III.34

Bellmann, Dennis: VII.60

Bello, Bruno: III.12, III.24

Benito-Sipos, Jonathan: II.12

Benton, Richard: VII.40

Berg, Christian: VII.11

Berger, Christian: II.4, III.13, III.14

Berger, Sandra: III.7

Berra, Erkosar: III.4

Bettencourt da Cruz, Alexandre: I.2

Bhar, Debjani: V.5

Bilen, Julide: VI.12

Birkholz, Oliver: III.14

Birman, Serge: I.5, V.9, V.42, VI.46, VII.8

BIRMAN, Serge: VII.27

Birse, Ryan T.: VI.47

Blau, Justin: IV.P

Bloch, Alice: V.11

Bogdan, Sven: V.17, V.20

Boll, Werner: III.2, III.9, VI.29, VII.9, VII.34

Borst, Alexander: VI.44

Bortnick, Rachel: VI.13

Borycz, Jolanta: V.41

Bosmans, Inge: I.38

Botella, Jose A.: I.4, I.29

Botella, José A.: I.15 
Botella, Jose Antonio: I.31

Boto, Tamara: IV.6

Boulanger, Ana: III.10

Brand, Andrea: I.27, II.3, VI.59

Brand, Andrea H.: II.8, III.35

Bredendiek, Nico: III.15

Brembs, Björn: VII.12

Brierley, David: VI.14

Briggs, Laura: V.12

Brindle, J: I.8

Brochtrup, Anna: VI.15

Brüsselbach, Florian: V.10

Buch, Susanne: VIII.4

Bucher, Dan: VII.54

Buchner, Erich: V.11, V.22, V.27, V.35, VII.8, VII.42,

VII.54

Buescher, Marita: II.13

Busch, Sebastian: VI.16

Cachero, Sebastian: VI.17

Cagan, Ross: II.9

Calamita, Piera: I.9

Callaerts, Patrick: I.19, I.24, I.38, III.19, III.22, VII.72

Canal, Inmaculada: III.27, VII.19

Cantera, Rafael: V.33

Carhan, Ahmet: VII.26

Carlson, John: III.6

Carlson, John R.: VI.9

Carlsson, Mikael A.: VI.19

Carmeliet, Peter: I.19

Carmena, Ana: II. 2

Cassar, Marlène: I.5

Ceriani, M.Fernanda: VI.39

Chan, Wei Hao: II.13

Chang, Kaichen: II.P

Charroux, Bernard: I.9

Chaudhary, Kokil: III.11, III.16, III.23

CHAUDY, Sylvie: VII.10

Chein, Cheng-Ting: V.3

Chélot, Elisabeth: VII.14, VII.33

Chen, Anna: II.23

Chen, Chien-Kuo: V.3

Chen, Ko-Fan: VII.36

Chen, Yi-chun: VII.13, VII.43

Cheng, Louise: III. 17

Cheng, Mei-Hsin: VI.36

Chernyk, Yaroslava: I.37

Chia, William: II.P, II.13

Chotard, Carole: III.18

Chou, Ya-Hui: VI.4

Christiansen, Frauke: V.37

Christiansen-Engelhardt, Frauke: V.13

Chuntao, Dan: VII.45

Cibic, Osman: I.35

Clements, Jason: III.19
Clouet, Chris: III.10

Collins, Ben: IV.P

Cooper, Robin: VI.53

Coulom, Hélène: I.5, VII.8

COULOM, Hélène: VII.27

Cusumano, Paola: VII.14

C. van der Plas, Mariska: V.39

Cziko, Anne-Marie: VI.21

Dahanukar, Anupama: VI.9

Dajas-Bailador, Federico: V.P

Das, Abhijit: II.26

Dascenco, Dan: VI.13

DasGupta, Shamik: VIII.3

Davis, Graeme: V.12

de Belle, J. Steven: VII.4, VII.61

de Jong, Anja: VI.30

de Jong, Eiko K.: I.12

Dekker, Teun: VII.23, VII.50

Deng, Ying: IV.4

Depner, Harald: V.37

Des, Claude: III. 8

Desplan, Claude: VII.66

De Strooper, Bart: I.16

Diao, Fengqiu: VI.33, VII.49

Díaz-Benjumea, Fernando J.: II.6

Diegelmann, Soeren: VI.18

Dierich-Schmitt, Eva: V.27

Ditzen, Mathias: VII.37

Domingos, Pedro: I.13

Drapeau, Mark D.: IV.P

Drummond, James: V.40

Dunkelberger, Brian: VII.4

DUPAS, Stéphane: VII.10

Dura, Jean-Maurice: III.10, VI.41

Dusik, Verena: VII.15

Dyba, Markus: V.15

Eder, Sascha: II.14

Edwards, Alexis: VII.72

Edwards, Tara N.: V.2

Effertz, Thomas: I.34, VI.26, VII.25

Egger, Boris: II.8, III.35

Ejima, Aki: VI.5, VII.16

Elliott, Chris: V.14

Elliott, Chris. J.H: VII.22

Emes, Richard: V.8

Endo, Keita: II.15

Enell, Lina: V.7

Enell, Lina E.: VI.19

Engelen, Daniel: III.20

Eschbach, Claire: VII.17

Estacio, Alicia: II.12

Evers, Felix: VI.35

Evers, Jan Felix: VI.2

Fabrice, LAGASSE: VII.18 
Fanto, Manolis: I.9

Fenton, Janine: VI.1, III.26

Fiala, André: VII.25

Fiala, André: VI.26, VI.27, VII.58, VII.60

Finster, Tamara: V.25

Fischbach, Karl: II.9

Fischbach, Karl-Friedrich: II.21, III.11, III.23

Fischbach, K.F: III.16

Fischer, Anja: II.2

Flandre, Adrien: III.10

Forero, Manuel: III.21, III.26

Forero, Manuel G.: VI.1

Fouquet, Wernher: V.13, V.15, V.37

Fradkin, Lee: V.38

Fradkin, Lee G.: VI.41

Fradkin, L.G: VI.30

Francis, Carmen: III.19

Franz, Mirjam: I.7

Frederic, MERY: VII.18

Freer, Stephanie: VII.4

Fricke, Robert: V.17

Frydman-Marom, Anat: I.3

Füger, Petra: V.25

Fukui, Ai: V.18

Funk, Natalja: VII.54

Gómez, Sara: III.26

Göpfert, Martin C.: VII.25

Galizia, Giovanni: VI.51

Gao, Shuying: VI.50

Garcia-Alcover, Irma: I.14

Garcia-Lopez, Amparo: I.14

Gazit, Ehud: I.3

Gendre, Nanae: VI.7, VII.48, VII.57, VII.62

Gentile, Carla: VI.20

Georg, steffes: VI.24

Gerber, Bertram: V.27, VII.17, VII.42, VII.43, VII.47, VII.54

Gerber, Betram: VII.13

Gervasi, Nicolas: VII.3

G. Fradkin, Lee: V.39

Ghezzi, Alfredo: VI.5

Giangrande, Angela: III.4

Giebultowicz, Jadwiga: I.23

Giesecke, Astrid: VI.20

Gisselmann, Günter: III.15

Glaser, Franz: VI.20

Gmeiner, Florian: I.15

Godenschwege, Tanja: I.38, V.30

Goellner, Bernd: V.6, V.19

Gohl, Christina: V.17, V.20

Gold, Kathrina S.: II.8

Goldman, Aaron: III.6

Gomez-Diaz, Carolina: IV.6, IV.7

Gonçalves, Ricardo: I.38
Gonzalo, Alicia: III.27, VII.19

Goossens, Tim: III.22

Göpfert, Martin C.: I.26, I.34, VI.26

Gorbashov, Anton: VI.57

Górska-Andrzejak, Jolanta: V.21

Götz, Kerstin: III.23

Gould, Alex P: III.17

Gouzi, Jean: VII.20

Gowers, Kate: I.28

Grace, Patricia: VI.33

Grammenoudi, Sofia: I.32

Grant, Jennifer: III.17

Grant, Seth: V.8

Green, David: V.7

Grevelhörster, Astrid: V.17

Griffith, Leslie: VI.5, VII.16

Grygoruk, Anna: V.41

Gu, Chun-Jing: VI.1

Guenter, Gisselmann: IV.4

Guignard, Thomas: III.10

Gundelfinger, Eckart D.: V.28

Haba, Daisuke: VII.21

Haddad, Dominik: I.16

Haessler, Ulrike: V.P

Hakeda-Suzuki, Satoko: III.7

Halder, Partho: V.22

Hallay, Natalia: V.25

Hampel, Stefanie: I.35

Han, Kyung-An: I.6, VII.57

HAN, Kyung-An: VII.27

Hansson, Bill: VII.23, VII.50

Hansson, Bill S.: VI.32

Harada, Eriko: VII.21

Hardie, Roger: IV.10

Harris, Kerri -Lee: III.31

Hartenstein, Volker: VI.48

Hasan, Gaiti: IV.3

Hassan, Bassem: V.44

Hassan, Bassem A.: I.22, VI.31

Hatt, Hanns: III.15, IV.4

Haussmann, Irmgard: V.4

Heale, B: I.8

Hebbar, Sarita: I.1

Heino, Tapio: III.25

Heino, Tapio I: I.25

Heisenberg, Martin: V.27, V.45, VI.11, VI.27, VII.53, VII.55, VII.59

Helfrich-Förster, Charlotte: VII.69

Helfrich-Foerster, Charlotte: VII.15

Helfrich-Förster, Charlotte: VI.22, VII.7, VII.32, VII.51, VII.67

Hens, Korneel: III.19

Herrero, Pilar: VII.39

Heß, Moritz: III.23 
Hidalgo, Alicia: III.21, III.26, VI.1

Hill, Julia: V.14, VII.22

Hillebrand, Jens: VI.21

Hindle, Samantha: I.17

Hirsh, Jay: I.21, VII.8

Hirth, Frank: I.40, II.22

Ho, Thi Thu Cuc: V.23

Hodge, James J.: IV.8

Hofbauer, Alois: V.22, VI.22

Hortsch, Michael: III.22

Hosamani, Ravikumar: I.18

Hovemann, Bernhard: V.45

Hovemann, Bernhard T.: V.10, V.41

Huber, Thomas: VII.40

Hummel, Thomas: III.5, VI.3, VI.42, VI.52

Humphrey, Dickon: I.40

Ibba, Irene: VII.23

Iche, Magali: VI.46

Iché, Magali: I.5, V.42, VII.8

Ignell, Rickard: VII.24

Inagaki, Hidehiko: VII.25

Inaki, Mikiko: V.18

Isaac, Elwyn: VII.26

Isabel, Guillaume: VII.3

ISABEL, Guillaume: VII.27

Istvan, Nagy: III.4

Ito, Kei: II.15, II.26, VI.8, VI.P, VI.16, VI.37, VI.45,

VII.3, VII.25, VIII.1

Itoh, Taichi Q: VII.28

Izergina, Natalya: III.24

Jackson, Rob: VII.29

Jacobs, An: I.38

Jansen, Sebastian: II.17

Janssens, Els: I.19

Jauch, Mandy: V.11

J.Diaz-Benjumea, Fernando: II.12

Jean-Marc, DEVAUD: VII.18

Jefferis, Gregory: VI.17

Jeibmann, Astrid: I.20

Jenett, Arnim: VI.23

Jiang, Yanrui: III.2

Johannes, Schindelin: VIII.2

Johard, Helena A.D.: VI.19

Jordanova, Albena: I.38

Joseph, Gen: I.13

Junfeng, Zheng: VI.24

Juusola, Mikko: VI.56, VII.2

Kahsai Tesfai, Lily: VI.25

Kaiser, Andrea: I.21

Kallijärvi, Jukka: III.25

Kamikouchi, Azusa: VI.26, VII.25

Kanellopoulos, Alexandros K.: VII.30

Kang, Yuan Yuan: III.22

Kannan, Ramakrishnan: II.4
Kapustjanskij, Alexander: VI.27

Karim, MD. Rezaul: II.10

Karlsson, Daniel: II.5, II.6

Kasprowicz, Jaroslaw: V.34, V.44

Katanaev, Vladimir: VI.51

Kato, Kentaro: III.21, III.26

Kawasaki, Fumiko: V.24, V.32

Keegan, Liam: I.8

Kei, Ito: VI.11

Kellner, Robert: V.15

Kern, Jeannine: V.25

Ketelhut, Manuela: II.23

Kherad Pezhouh, Maryam: I.6

Khorramshahi, Omid: IV.9

Kienitz, Bastian: VII.31

Kim, A Young: V.26

Kim, Won Tae: V.26

Kim, Yong-Sun: V.31

Kim, YoonJung: V.31

Kim, Young-Cho: I.6

Kistenpfennig, Christa: VII.32

Kittel, Robert: V.28

Klaembt, Christian: III.36

Klämbt, Christian: I.20, III.3, III.20, III.32, III.33, V.17, V.20

Klarsfeld, André: VII.14, VII.33

Klebes, Ansgar: I.7

KN, Chandrashekar: I.18

Knapek, Stephan: V.27

Knoblich, Juergen: II.2

Knust, Elisabeth: V.28

Kobler, Oliver: V.28

Koch, Iris: V.6

Koch, Marta: I.22

Kochinke, Korinna: I.12

Koh, Young Ho: V.23, V.26, V.31

Kohsaka, Hiroshi: V.29

Kolodziejczyk, Agata: VI.28

Köper, Martina: VII.60

Körner, Jorg: V.37

Kowalkowski, Victoria: VI.18

Kramer, Jamie M.: I.12

Kramer, Stella: V.25

Krantz, David E.: V.41

Kraut, Rachel: I.1

Kretzschmar, Doris: I.2

Krishnan, KS: V.5

Krishnan, Natraj: I.23

Krohne, Georg: VII.54

Krstic, Dimitrije: VI.29, VII.9, VII.34

Kudumala, Shirisha: V.30

Kuenen, Sabine: V.34, V.44

Kuhlmann, Britta: III.5

Kumar, Abhilasha: II.18 
Kumar, Vimlesh: V.5

Kume, Kazuhiko: VII.35, VII.63, VII.65

Kume, Kazunori: VII.8

Kume, Shoen: VII.35, VII.63, VII.65

Kunes, Sam: VII.45

Kungl, Theresa: VI.49

Kunz, T: II.19

Kunz, Tomas: III.31

Kupenova, Petia: IV.10

Kwon, Jae Young: VI.9

Lahaye, Liza: VI.30

Landgraf, Matthias: III.29, VI.2, VI.18, VI.35

Langen, Marion: VI.31

Lanskron, Johannes: VII.36

Laridon, Bram: I.24

Larsch, Johannes: VII.37

Laverty, Todd R.: VI.23

Lavista-Llanos, Sofia: VI.32

Learte, Anabel: III.21

Lee, Chi-Hong: V.7

Lee, Dae Weon: V.23, V.26, V.31

Lee, Hyun-Gwan: I.6

Lee, Jihye: IV.2

Lee, Sang-Hee: V.31

Lehmann, Fritz-Olaf: VII.56

Leiss, Florian: V.13, VI.6

Leptourgidou, Flora: VII.38

Levy, Michal: I.3

Leyssen, Maarten: I.16

Lichtneckert, Robert: II.26, III.12

Lim, Angeline: I.1

Lin, Wei-Hsiang: IV.5

Lindström, Riitta: I.25

Linne, Viktoria: II.17

Linneweber, Gerit Arne: II.21

Llorens, Jose Vicente: I.31

Lohse, Martin: VI.27

Lopez, Begoña: I.29

López Arias, Begoña: VII.39

Losada, María: II.11, III.27

Louis, Matthieu: VII.40

Lu, Simon Q.: I.26

Luan, Haojiang: VI.33, VII.49

Ludlow, Zoe: II.22

LuLee, Chi-Hon: VI.50

Luo, Liqun: VI.4

Lutas, Andrew: V.32

Luu, Si-Hong: III.7

M, Muralidhara: I.18

Mackay, Trudy: VII.72

Maeyama, Yuko: VII.45

Magwire, Mike: VII.72

Maidment, Nigel T.: V.41

Maier, Dieter: II.23
Maksymiv, Dariya: I.37

Malik, Bilal: V.8

Malpel, Sébastien: VII.33

Mandemakers, Wim: I.16

MANIERE, Gérard: VII.10

Marley, Richard: VI.34

Martin, Fernando: IV.7

Martin, Heisenberg: VIII.2

Martin, Jean-Rene: VI.38

Martín-Joven, Jorge: VII.39

Maruhn, Sinje: VII.32

Masek, Pavel: VII.41

Masuda-Nakagawa, Liria M: VI.7

Masuyama, Kaoru: V.7

Matsumoto, Akira: VII.28

Matsuo, Takashi: VII.21

Maurange, Cedric: III.17

Mauss, Alex: VI.35

Mayor, Ugo: I.27

McGurk, L: I.8

McKellar, Claire: VI.36

McQuilton, Peter: VI.1

Mehnert, Kerstin I.: V.33

Meinertzhagen, Ian: VI.50

Meinertzhagen, Ian A.: V.2, V.41

Mejia, Monica: V.30

Melcher, Christoph: VIII.4

Mentzel, Benjamin: VI.49

Mertel, Sara: V.13, V.15, V.37

Mertens, Cordula: VII.32

Mery, Frederic: VII.5

Mettler, Ulrike: II.14, II.17

M E Winther, Åsa: VI.25

Michard-Vanhée, Christine: VI.22

Michels, Birgit: VII.42, VII.54

Middleton, Adam: V.14

Mihaly, Jozsef: V.P

Milan, Petrovic: VI.15

Milton, Valerie: I.28

Minocha, Shilpi: III.9

Mishra, Dushyant: VII.13, VII.43

Miskiewicz, Katarzyna: V.34

Mitsuyoshi, Madoka: VII.35, VII.63

Miyazaki, Takaaki: VI.37

Mizrak, Dogukan: IV.P

Mizuguchi, Kenji: VI.1

Molina, Isabel: II.11, III.27, VII.19

Moltó, Maria Dolores: I.31

Monedero, Ignacio: I.29, III.27

Moore, David: I.6

Morais, Vanessa: I.16

Moreau, Yves: VI.31

Moressis, Anastasios: VII.20, VII.44

Morgan, Matthew: I.22 
Moris, Marta: II.12

Muñoz-Soriano, Veronica: I.30

Murakami, Satoshi: II.28, VII.45

Murmu, Meena: VI.38

Murphey, Rod: V.30

Murphy, Christine: VI.23

Nadrowski, Björn: I.26

Nakamura, Akira: VI.21

Nakao, Keiko: II.24

Nangle, Leslie: I.38

Napoletano, Francesco: I.9

Nassel, Dick: V.7, VI.28

Nässel, Dick R: VII.24, VI.19, VI.47

Navarro, Juan Antonio: I.31

Navarro-López, Josep: I.30

Negele, Julia: III.28

Neuhaus, Eva M.: III.15, IV.4

Neuser, Kirsa: VII.8, VII.46

Ng, Fanny: VII.29

Ngo, Teri: VI.23

Niañko, Ewelina: V.21

Niehues, Sven: VI.3

Nieratschker, Vanessa: V.11

Niewalda, Thomas: VII.47

Nikolaev, Viacheslav: VI.27

Nisoli, Ilaria: I.9

N. Noordermeer, Jasprina: V.39

Nolan, Nicole: VII.4

Noll, Markus: III.2, III.9, VI.29, VII.9, VII.34

Noordermeer, Jasprien: V.38

Noordermeer, Jasprina N.: VI.41

Noordermeer, J.N.: VI.30

Norga, Koen: I.24, I.38, VII.72

North, Annemarie: III.29

Nose, Akinao: V.18, V.29

Nuwal, Tulip: V.35

Occhi, Simona: I.9

O'Connell, M.A.: I.8

Ogueta- Gutierrez, Maite: I.21

Oh, Eugene: V.40

O'Hare, Megan: I.39

Ohler, Stephan: III.7, III.30

O'Kane, Cahir J: VI.7

Okano, Hideyuki: II.24

Oliva, Carlos: VI.39

Ooms, Tinne: I.38

Oortveld, Merel A.W.: I.12

Orban, Komonyi: III.4

Ordway, Richard: V.24

Orihara-Ono, Minako: II.24

Oswald, Matt: V.12

Oswald, Matthew: V.36

Ovezmuradov, Guvanch-Murad: I.34

Owald, David: V.13, V.15, V.37
Palgi, Mari: I.25

Pankratz, Michael: VI.40

Pankratz, Michael J.: VIII.4

Papanikolopoulou, Aikaterini: I.32

Paricio, Nuria: I.30

Parker, Roy: VI.21

Partridge, Linda: I.P

Pauls, Dennis: VII.48, VII.57, VII.62

Paulus, Werner: I.20

Peabody, Nathan: VI.33, VII.49

Pendred, Julia: III.17

Pennack, Jenny: III.21, VI.1

Peters, Marc: VI.40

Petrova, Iveta M.: VI.41

Petrovic, Milan: VI.42, VI.52

Pettersson, Nina: I.33

Pfeiffer, Barret D.: VI.23

Pfitzenmaier, Johanna: VII.48, VII.57

Picot, Marie: VII.14, VII.33

Pilgram, Gonneke: V.38

Pimentel, Catarina: V.P

Pocklington, Andrew: V.8

Poeck, Burkhard: VII.46

Pollarolo, Giulia: III.22

Potikanond, Saranyapin: V.39

Powell, Sophie: III.26

Preat, Thomas: VII.3

PREAT, Thomas: VII.27

Preiss, Anette: II.23

Prieto Godino, Laura Lucia: VI.43

Prokop, Andreas: V.P

Pütz, Stefanie: V.10

Pym, Edward: VI.59

Pyza, El_bieta: V.21

Raabe, Thomas: VI.49

Raghu, Shamprasad Varija: III.23, VI.44

Rajeswaran, Pingkalai: I.34

Ramaswami, Mani: V.5, VI.21

Randlett, Owen: VI.2

Randlkofer, Barbara: VII.50

Rasse, Tobias M: V.25

Rathore, Kirti: VI.14

Reaume, Christopher J.: VII.5

Reichert, Heinrich: II.18, II.26, III.12, III.24

Reiff, Dierk F.: VI.44

Reisch, Dietmar: VII.54

Richardt, Arnd: VII.60

Richier, Benjamin: VII.14

Rickert, Christof: III.31, III.34

Rieger, Dirk: VII.51

Riemensperger, Thomas: I.5, VII.8

Rister, Jens: V.45

Ritze, Yvonne: I.21

Rival, Thomas: V.42 
Robinson, Iain: V.40

Rodrigues, Floriano: III.32

Rodrigues, Veronica: II.26

Rogulja-Ortmann, Ana: II.7

Roman, Gregg: VII.52

Romero-Calderón, Rafael: V.41

Root, Cory: V.7

Root, Cory M: VII.24

Rothig, Anne: I.16

Rouyer, François: VI.22, VII.8, VII.14, VII.33

Ruan, Hongyu: I.41

Ruben, Artero: I.11

Ruben, Marc: IV.P

Rubin, Gerald M.: VI.23

Ryu, Hyoje: VI.13

Saarma, Mart: I.25, III.25

Saito, Mai: V.42

Sakamoto, Erina: VII.35, VII.63

Sakmar, Thomas: VII.40

Salecker, Iris: III.P, III.18

SALLE, Jérémy: VII.10

Sanchez-Soriano, Natalia: V.P

Saratsis, Anastasios: I.7

Sareen, Preeti: VII.53

Sato, Makoto: II.28

Saumweber, Timo: VII.54

Schellens, Ann: III.19

Schenck, Annette: I.12

Scheper, Christoph: VI.3

Scherzer, Roni: I.3

Schimmel, Paul: I.38

Schlieder, Marlen: I.22

Schmid, Benjamin: VIII.2

Schmidt, Imke: III.33

Schmidt, Manuela: V.37

Schmucker, Dietmar: VI.13

Schnaitmann, Christopher: VII.55

Schneider, Andrea: I.35

Schnell, Bettina: VI.44

Schneuwly, Stephan: I.4, I.15, I.31, I.37

Scholz, Henrike: I.7, I.21, I.35

Schultz, Sebastian: I.36

Schützner, Peter: VII.56

Schwarz, Heinz: V.6

Schwärzel, Martin: VII.60

Scorrano, Luca: I.16

Scott, Kristin: VII.41

Seeds, Andrew: VII.6

Segal, Daniel: I.3

Sehadova, Hanna: VI.20

Seibert, Janina: II.25

Sejourne, Julien: VII.3

Selcho, Mareike: VI.16, VII.48, VII.57, VII.62

Semenov, Eugene: IV.10
Sen, Sonia: II.26

Serantes, Pablo: VII.39

Serway, Christine: VII.4

Sharma, Ankit: V.40

Shashidhara, L.S.: II.4, III.13

Shcherbakova, Oksana: I.37

Shinomiya, Kazunori: VI.45

Shiraki, Nobuaki: VII.63

Shirras, Alan: VII.26

Shirras, Christine: VII.26

Shyer, Amy: V.41

Sidhu, Harpreet: II.27

Siebert, Matthias: V.19

Sierralta, Jimena: V.28

Sierrralta, JImena: VI.39

Sigrist, Stephan: V.13, V.27

Sigrist, Stephan J: V.37, V.15, V.28

Silies, Marion: III.3, III.33

Simon, Anne F.: V.41

Simoni, Alekos: VI.20

Simpson, Julie: VI.12, VI.36, VII.6

Sinakevitch, Irina: V.9, V.42, VI.46

Singhal, Nidhi: VII.58

Sitaraman, Divya: VII.71

Skoulakis, Efthimios: I.32, VII.20, VII.38, VII.44

Skoulakis, Efthimios M.C.: VII.30

Smet, Joel: I.16

Smith, Brian H: VI.46

Snellinx, An: I.16

Söderberg, Jeannette A. E.: VI.47

Sokolowski, Marla B.: VII.5

Solanki, Narendra: VII.59

Soller, Matthias: V.4

Somers, Wayne G: II.P

Southall, Tony: II.3, VI.59

Sparrow, John: V.14

Sparrow, John. C: VII.22

Speicher, Stephan: II.2

Spindler, Shana: VI.48

Spletter, Maria: VI.4

Sprecher, Simon: III.8

Srahna, Mohammed: VI.31

Srivastava, Rajneesh: III.23

Stanewsky, Ralf: IV.8, VI.20, VII.36

Stark, Felix: VI.49

Steffes, Georg: III.5

Steinborn, Rafaela: VI.52

Steller, Hermann: I.13

Stensmyr, Marcus C.: VI.32

Stephan, Raiko: V.20

Stocker, Reinhard: VII.48, VII.57, VII.62

Stocker, Reinhard F: VI.7

Stopfer, Mark: VI.8

Storkebaum, Erik: I.38 
Störtkuhl, Klemens: VII.60

Strauss, Roland: VII.8, VII.11, VII.31, VII.46, VII.64, VII. 70

Suh, Joowon: VII.29

Sutcliffe, Ben: VI.1

Suter, Beat: I.33

Suzuki, Satoko: III.30

Suzuki, Takashi: III.7, III.30

Sweeney, Sean: I.17, V.14

Sweeney, Sean T: V.12, VII.22, I.28

Tabata, Tetsuya: II.28, VII.45

Tabone, Christopher J.: VII.61

Takahama, Kazuhiro: VII.35

Takahashi, Kuniaki: VII.28

Takemura, Shinya: VI.50

Tanaka, Nobuaki: VI.8

Tang, Ke: VII.26

Tang, Shiming: VII.2

Tanimoto, Hiromu: V.27, VI.11, VI.16, VII.42, VII.55

Tanimura, Teiichi: VII.28

Tate, Phillip: I.22

Tavosanis, Gaia: III.28, VI.6

Tear, Guy: I.39, VI.54

Technau, Gerd: II.4, II.7, III.31

Technau, Gerhard: III.34

Technau, Gerhard M.: II.25, III.14

Technau, Gerhard, M.: III.13

Technau, GM: II.19

Terriente, Javier: II.6

Thoma, Michael: VI.51

Thomas, Hummel: VI.15, VI.24

Thomas, Ulrich: V.28

Thor, Stefan: II.5, II.6, II.12

Thum, Andreas: VII.48, VII.57, VII.62

Timmerman, Vincent: I.38

Timofeev, Katarina: VI.52

Ting, Chun-Yuan: VI.50

Tobias, Schwarz: IV.9

Tomasi, Tatiana: III.7, III.30

Tomita, Jun: VII.35, VII.63, VII.65

Tonoe, Gaku: V.18

Torroja, Laura: I.29, VII.19

Travis, Mark: V.P

Triphan, Simon: VII.55

Triphan, Tilman: VII.55, VII.64

Tripodi, Marco: VI.35

Turiégano, Enrique: VII.19

Turner, Ashby: VI.53

Tuxworth, Richard: I.39

Ueda, Atsushi: V.43

Ueda, Hiroki R: VII.28

Ueda, Ryu: VII.28

Ueno, Taro: VII.35, VII.65

Uhlenbrock, Guido: V.41
Ukai-Tadenuma, Maki: VII.28

Umetsu, Daiki: II.28

Urbach, R: II.19

Urbach, Rolf: II.25

Urban, Joachim: II.14, II.17

Uytterhoeven, Valerie: V.44

van Bokhoven, Hans: I.12

Van Coster, Rudy: I.16

van den Brink, Daan: VI.54

Velikova, Nadya: IV.10

Venkiteswaran, Gayatri: IV.3

VERNIER, Philippe: VII.27

Verstreken, Patrik: I.16, V.34, V.44

VijayRaghavan, K: VI.14

Vilain, Sven: I.16

Vogler, Georg: III.14

Vogt, Nina: VII.66

Voigt, Aaron: I.4

Volkmann, Katrin: I.35

Volland, Dagmar: II.25

Volpi, Vera Giulia: I.9

Vömel, Matthias: VI.55

von Alpen, Desiree: VII.48

von Hilchen, Christian: III.34

von Trotha, Jakob W.: III.35

Vosshall, Leslie: VII.40

Vosshall, Leslie B.: VII.37

Waddell, Scott: VIII.3

Wang, Cheng: II.27

Wang, Hongyan: II.P, II.27

Wang, Howard: VI.33

Wang, Jing: V.7

Wang, Jing W: VII.24

Wardill, Trevor: VI.56

Webb, Barbara: VI.58

Wegener, Christian: VI.55, VI.57

Wegener, Stephanie: VII.54

Weiss, Linnea: VI.9

Wenderdel, Stefanie: V.17

Wentzell, Jill: I.2

Werner, Uschi: I.35

Wessnitzer, Jan: VI.58

Westermark, Gunilla: I.36

White, Benjamin: VI.33, VII.49

White, Kalpana: V.4

White, Katherine: I.40

White, Katherine E: II.22

Whitington, Paul: III.31

Whitmarsh, Alan: V.P

Wichmann, Carolin: V.28, V.37

Williams, Darren: VI.14

Wilson, Rachel: VI.4

Winther, Åsa M E: VII.24

Witte, Hanna: I.20 
W.M. de Jong, Anja: V.39

Wolf, Eva: VII.36

Wolf, Reinhard: VII.53, VII.59

Wolfram, Verena: VI.59

Wolstenholme, Adrian: IV.10

Wouda, Rene: VI.30

Wright, Duncan E.: IV.5

Wu, Chun-Fang: I.41, IV.2, V.43

Wuelbeck, Corinna: VII.15

Wülbeck, Corinna: VII.32, VII.51, VII.67

Wurst, Wolfgang: I.16

Wuytens, Gunther: III.22

Yaksi, Emre: VI.4

Yamamoto, Daisuke: VII.68

Yamazaki, Masako: VII.35, VII.63, VII.65

Yan, Jiekun: I.22

Yang, Xian-Lei: I.38
Yasugi, Tetsuo: II.28

Yee, Susan K.: V.41

Yoshii, Taishi: VII.7, VII.69

Young, Joanna: VI.60

Yusein, Shazie: IV.10

Yuva Aydemir, Yeliz: III.36

Zaepf, Bianca: VII.70

Zagaeski, Brendan: VII.45

Zaida, Garcia-Casado: I.11

Zars, Melissa: VII.71

Zars, Troy: VII.71

Zhang, Weiyi: IV.4

Zhu, Bangfu: VI.1

Ziegler, Anna: V.45

Zimmermann, Pascale: III.22

Zwart, Maarten: VI.2

Zwarts, Liesbeth: VII.72 